S E M I N A R I O G I U R I D I C O

D E L L A U N I V E R S I T À D I B O L O G N A CCCI

\author{
GIULIO CENTAMORE
}

\title{
CONTRATTAZIONE COLLETTIVA E PLURALITÀ DI CATEGORIE
}


S E M I N A R I O G I U R I D I C O

D E L L A U N I V ERS I T À D I B O L O G N A CCCI

\author{
GIULIO CENTAMORE
}

\title{
CONTRATTAZIONE COLLETTIVA E PLURALITÀ DI CATEGORIE
}


Bononia University Press

Via Ugo Foscolo 7, 40123 Bologna

tel. (+39) 051232882

fax $(+39) 051221019$

www.buponline.com

e-mail: info@buponline.com

Quest'opera è pubblicata sotto licenza Creative Commons BY-NC-SA 4.0

ISSN 2283-916X

ISBN 978-88-6923-567-2

ISBN online 978-88-6923-568-9

DOI 10.30682/sg301

Prima edizione: maggio 2020 
D E L L A U N I V ERS I T À D I B O L O G N A

Per la presente monografia la Giunta di Dipartimento ha nominato la seguente Commissione di lettura:

Andrea Lassandari (Alma Mater Studiorum - Università di Bologna), per il Settore Scientifico Disciplinare IUS/07 Diritto del Lavoro

Sandro Mainardi (Alma Mater Studiorum - Università di Bologna), per il Settore Scientifico Disciplinare IUS/07 Diritto del Lavoro

Carlo Zoli (Alma Mater Studiorum - Università di Bologna), per il Settore Scientifico Disciplinare IUS/07 Diritto del Lavoro 
Ai miei genitori e a zia Mary 


\section{CAPITOLO I}

\section{LIBERTÀ SINDACALE E CATEGORIA CONTRATTUALE}

Sommario: 1. Il problema della categoria. - 2. Pluralità di significati del lemma categoria nel diritto sindacale. -3 . Categoria professionale e categoria sindacale. -4 . Categoria contrattuale. -5 . Le tipizzazioni dell'autonomia collettiva. 6. Principio di libertà sindacale e definizione delle unità negoziali. - 6.1. La giurisprudenza costituzionale sulla legge Vigorelli. - 6.2. L'affermazione di una nozione volontaristica di categoria. - 7. La sovrapposizione tra gli ambiti di applicazione dei contratti collettivi di categoria: un fenomeno relativamente nuovo. - 8. Inquadramento delle questioni da affrontare e struttura del lavoro.

\section{Il problema della categoria}

Il tema della categoria - che già cinquanta anni fa Giugni definiva «suggestivo e antico» ${ }^{1}$ - è dai più considerato particolarmente complesso $^{2}$, se non un puzzle logico-giuridico ${ }^{3}$, o persino uno dei nodi permanenti della materia ${ }^{4}$ : uno di quei problemi, cioè, che so-

1 G. Giugni, Osservazioni sulle sentenze n. 70 e 106 della Corte costituzionale, in Giur. Cost., 1963, p. 828.

2 P. Lambertucci, Area contrattuale e autonomia collettiva, in Dir. Lav. Rel. Ind., 1999, p. 284.

3 V. PAPA, Verso una rappresentanza misurata? Strumenti e metodi di misurazione della rappresentatività datoriale, in Dir. Lav. Rel. Ind., 2017, p. 38.

4 M. BARBIERI, La contrattazione collettiva nazionale oggi: caratteri, metamorfosi, criticità, in A. Lassandari, F. Martelloni, P. Tullini, C. Zoli (a cura di), 
lo temporaneamente possono «scomparire nella massa della materia trattata [...] per l'affollarsi di questioni particolari, [ma che] a causa dell'importanza che rivestono per la scienza tornano di continuo alla superficie ed esigono attenzione» ${ }^{5}$. Ciò non dovrebbe stupire, se si considera la sorprendente varietà di prospettive e, per così dire, di gradazioni, con le quali il tema de quo si è riproposto, nel corso della (ormai più che) «semisecolare singolare vicenda del nostro diritto sindacale eternamente provvisorio» ${ }^{6}$.

Vi è la questione per definizione irrisolta di se e come dare attuazione all'art. 39 Cost. Una legge che attribuisse effetti erga omnes ai contratti collettivi non potrebbe eludere il nodo, da una parte, dell'adozione di meccanismi mediante i quali definire le unità negoziali della contrattazione collettiva (comma $4^{\circ}$ ) e, dall'altra, della conciliabilità di tali meccanismi con il principio di libertà sindacale $\left(\text { comma } 1^{\circ}\right)^{7}$.

Si ricorderà che all'epoca della ricostruzione post-costituzionale del diritto sindacale ${ }^{8}$, la nozione di categoria è stata al centro di uno dei "sommovimenti" decisivi per la cultura della materia, per opposizione o, se si vuole, in polemica con i retaggi del regime corporativo ${ }^{9}$. Da allora, non è più stato messo seriamente in discussione l'assunto che una nozione preconcetta di categoria non sia ricavabile dall'ordinamento giuridico ${ }^{10}$. Eppure, vi è talvolta l'impressione che il dibattito sulla nozione di categoria non sia del tutto sopito: non ne rappresenta forse una

La contrattazione collettiva nello spazio economico globale, Bologna, Bononia University Press, 2017, p. 5.

5 R. Flammia, Problemi circa l'ambito di applicazione dei contratti collettivi, in AA.VV., Categorie professionali e contratti collettivi, Suppl. Notiz. Giur. Lav., Roma, Assicredito, 1990, p. 21.

6 G. Pera, La contrattazione collettiva di diritto comune e l'art. 2070 c.c., in Giust. Civ., 1997, p. 1203.

7 Cfr. G. Pera, Problemi costituzionali del diritto sindacale italiano, Milano, Feltrinelli, 1960, pp. 51-68.

8 P. Ichino, I primi due decenni del diritto del lavoro repubblicano, I. Dalla liberazione alla metà degli anni '50, II. Dalla metà degli anni '50 alla legge sui licenziamenti individuali, in Riv. It. Dir. Lav., 2007, I, pp. 221 ss., 249 ss.

9 G.F. Mancini, Libertà sindacale e contratto collettivo "erga omnes", in Riv. Trim. Dir. Proc. Civ., 1963, p. 570 ss. ora con il titolo Il problema dell'art. 39 (libertà sindacale e contratto collettivo erga omnes) in ID., Costituzione e movimento operaio, Bologna, Il Mulino, 1976, p. 133 ss.

10 U. Romagnoli, Il contratto collettivo d'impresa, Milano, Giuffrè, 1963, pp. 40-45. 
prova il fatto che sia stata in questi anni discussa, in dottrina, la legittimità costituzionale dell'art. 8, 1. n. 148/2011, nella parte in cui prevede l'efficacia generale dei contratti collettivi di prossimità ${ }^{11}$ ?

Vi è altresì la prospettiva - non più attuale, se non per gli insegnamenti che se ne possono trarre - della legge cd. Vigorelli (n. 741/1959), che ha posto alla dottrina e alla giurisprudenza dell'epoca, anche costituzionale ${ }^{12}$, questioni di elevata complessità, relativamente alla definizione del campo di applicazione delle discipline collettive recepite nei decreti delegati ${ }^{13}$.

Fino alla storica sentenza n. 2665/1997 delle Sezioni Unite della Cassazione ${ }^{14}$, il tema de quo è stato teatro di un episodio, invero, tra i più controversi, di dissociazione tra formante dottrinale e giurisprudenziale, in merito all'applicabilità dell'art. 2070 c.c. ai contratti collettivi di diritto comune. Tuttavia, il consolidamento, nella giurisprudenza successiva ${ }^{15}$, dell'orientamento accolto dalle Sezioni

11 Il problema ha, come noto, origine dai dubbi di legittimità costituzionale che solleverebbe qualsiasi legge o atto avente forza di legge che estendesse gli effetti dei contratti collettivi «con forme e procedimento diversi da quelli previsti dall'art. 39 Cost.» (C. cost. 19 dicembre 1962, n. 106, in Giur. Cost., 1962, p. 1408, note V. Crisafulli, M. Mazziotti, M. Conti, Mass. Giur. Lav., 1962, p. 648, nota G. Pera, Riv. Dir. Lav., 1963, II, p. 23, nota V. Carullo). Secondo un primo orientamento, tuttavia, il problema non avrebbe motivo di porsi in relazione all'art. 8, cit., dal momento che la norma costituzionale impedirebbe di estendere l'efficacia soggettiva (in modi diversi da quelli previsti dall'art. 39 Cost.) dei soli contratti collettivi di settore (nell'esperienza italiana: nazionali e provinciali); secondo altri, invece, una tale interpretazione non sarebbe ricavabile dalla norma costituzionale, se non finendo per ricadere nel preconcetto di una definizione aprioristica della categoria: l'impedimento costituzionale ad estendere erga omnes, per legge, l'efficacia soggettiva dei contratti collettivi sussisterebbe, perciò, non soltanto per i contratti collettivi di settore, ma anche per quelli di livello aziendale. Sul tema, cfr. V. LeCCESE, Il diritto sindacale al tempo della crisi. Intervento eteronomo e profili di legittimità costituzionale, in Dir. Lav. Rel. Ind., 2012, pp. 500-503, L. LAZZERONI, La regolazione per legge del contratto collettivo aziendale alla luce del sistema costituzionale, Torino, Giappichelli, 2017.

12 Specialmente, C. cost. 15 maggio 1963, n. 70, 20 giugno 1963, n. 106, entrambe in Giur. Cost., 1963, pp. 585, 820, nota G. GIUGNI, ivi, p. 822 ss.

13 Sul tema, cfr. D. GUERRIERI, La categoria nell'ordinamento giuridico del lavoro, Milano, Giuffrè, 1963; v. altresì i contributi raccolti in AA.VV., La categoria e la contrattazione collettiva, Milano, Giuffrè, 1964.

14 Cass. SSUU 26 marzo 1997, n. 2665, in Giust. Civ., 1997, p. 1203, nota G. Pera, Mass. Giur. Lav., 1997, p. 739, nota R. Scognamiglio, Giur. It., 1998, p. 916, nota M. Marazza.

15 Tra le molte, Cass. 8 maggio 2008, n. 11372, in Giust. Civ. Mass., 2008, p. 683, Cass. 13 luglio 2009, n. 16340, in Giust. Civ. Mass., 2009, p. 1087, Cass. 18 
unite non ha impedito che continuasse a porsi il problema di una selezione, mediante criteri oggettivi, dei contratti collettivi da assumere quale parametro del trattamento economico e/o normativo, nei casi di rinvio dalla legge all'autonomia collettiva ${ }^{16}$. In altri termini, quella giurisprudenza non ha impedito che si ponesse nuovamente il problema della nozione di categoria di cui si sarebbe dotato il legislatore ${ }^{17}$.

Per non dare che un ultimo, ma non meno rilevante, elemento di contesto: la definizione dei comparti, ai fini della contrattazione collettiva per il settore pubblico, rappresenta uno dei più delicati momenti di contatto, in questa materia, tra intervento dei pubblici poteri e sfera di autonomia delle parti sociali. La dottrina lo aveva evidenziato fin dalla legge-quadro n. 93/1983 ${ }^{18}$ e, dagli anni Novanta in poi, le vicende della contrattazione collettiva lo hanno confermato ${ }^{19}$, ma è con la coatta riduzione a quattro del numero dei comparti, prevista dalla cd. riforma Brunetta del 2009, che i nodi sembrano essere venuti al pettine ${ }^{20}$.

\section{Pluralità di significati del lemma categoria nel diritto sindacale}

Al fine di orientarsi in questo groviglio di discipline gius-sindacali "in stile italiano", giova per prima cosa chiarire meglio cosa s'intenda quando si utilizza il lemma categoria.

In un'accezione basilare o, se si preferisce, generalissima, esso

dicembre 2014, n. 26742, in Pluris. Nella giurisprudenza di merito, Trib. Taranto, 22 marzo 2010, in Lav. Giur., 2010, p. 736, nota F. Collia.

16 A. Bellavista, La clausola sociale dell'art. 36 st. lav. e l'art. 2070 c.c., in Riv. It. Dir. Lav., 1999, II, p. 462 ss.

17 Cfr. M. Magnani, Riflessioni sulla misurazione della rappresentanza datoriale nell'ordinamento statale e intersindacale, in WP CSDLE “Massimo D'Antona”.IT, 376/2018, pp. 9-10, L. IMBERTI, Trattamento economico minimo (del socio lavoratore) e c.c.n.l. parametro: chi individua la categoria ed il perimetro della stessa?, in Labor, 2019, pp. 404-414.

18 A. Orsi Battaglini, Della quadratura di molti circoli, in Pol. Dir., 1984, p. 11.

19 M. BARBIERI, Problemi costituzionali della contrattazione collettiva nel lavoro pubblico, Bari, Cacucci, 1997, p. 419 ss.

20 C. Zoli, La struttura della contrattazione collettiva nel lavoro pubblico, in L. Fiorillo, A. Perulli (dir. da), Il nuovo diritto del lavoro, 1, Il lavoro alle dipendenze delle amministrazioni pubbliche, Torino, Giappichelli, 2013, p. 165. 
allude alla rappresentazione di una porzione ideale o materiale della realtà, mediante la costruzione di una serie di relazioni tra sequenze linguistico-concettuali e dati di esistenza oggettiva ${ }^{21}$ : vi si potrebbe alludere in termini di "classi di realtà". Corrispondente a locuzioni quali serie, classificazione, raggruppamento e così via, "categoria" potrebbe a ben vedere riferirsi a qualsiasi partizione dotata di rilevanza per la materia, in base al contesto nel quale è utilizzato, agli attributi che lo qualificano o alle sue specificazioni. Categoria professionale, categoria sindacale, categoria contrattuale, interesse di categoria, autotutela di categoria, rivendicazione di categoria, sindacato di categoria, contratto di categoria (e si potrebbe continuare) sono tutte nozioni che sollevano difficoltà terminologiche e concettuali di non poco momento ${ }^{22}$. Non stupisce - notava tempo addietro Tarello - che tale vocabolo sia adoperato in molti luoghi dell'ordinamento giuridico senza essere definito, o che dalla dottrina e dalla giurisprudenza sia sovente presupposto, e solo di rado precisato, con esiti, peraltro, incerti e controvertibilii ${ }^{23}$.

Ad esempio, in occasione della celebre sentenza n. 123 del 1962 sullo sciopero per fini non contrattuali ${ }^{24}$, la Corte costituzionale adoperò il vocabolo categoria in una varietà di accezioni, non sempre, peraltro, in modo perspicuo, come osservava all'epoca Pera ${ }^{25}$. Il giudizio a quo prendeva le mosse da un fatto di «abbandono collettivo del lavoro effettuato dagli appartenenti a quattro categorie di personale»; era discusso se fosse o meno legittimo «escludere o limitare il diritto garantito dall'art. 40 in confronto a quella determinata categoria di prestatori»; si poneva un problema di confronto tra «interessi generali assolutamente preminenti rispetto agli altri collegati

21 R. WARDY, Categories, in Routledge Encyclopaedia.

22 M. Dell'Olio, Ancora sulla rappresentatività, e su maggioranza e proporzionalità, organizzazione sindacale, categoria, nello statuto dei lavoratori e nel sistema, in Giur. Cost., 1988, II, p. 1433 ss.

23 G. TARELlo Teorie e ideologie nel diritto sindacale, Milano, Edizioni di Comunità, 1967 , p. 45 testo e nota 50, ma passim.

24 Cfr. F. CARINCI, Il conflitto collettivo nella giurisprudenza costituzionale, Milano, Giuffrè, 1971, p. 50 ss.

25 G. Pera, Lo sciopero di solidarietà nelle più recenti concezioni, in Riv. Giur. Lav., 1964, I, p. 1 ss. La sentenza citata nel testo è ovviamente C. cost. 28 dicembre 1962, n. 123, in Dir. Lav., 1963, II, p. 198. 
all'autotutela di categoria»; si affermò, inoltre, che «non è contestabile la sussistenza di interessi comuni a intere categorie di lavoratori»e che a determinate condizioni è legittima la sospensione del lavoro in appoggio a «uno sciopero già in via di svolgimento, ad opera di lavoratori appartenenti alla stessa categoria dei primi scioperanti»; infine, in uno dei passaggi ancora oggi più controversi della motivazione ${ }^{26}$, si sostenne che spetta al giudice del merito verificare «il grado di collegamento fra gli interessi economici di cui si invoca la soddisfazione ed, in relazione ad esso, determinare l'ampiezza da assegnare al complesso categoriale formato dai titolari degli interessi stessi».

Non si andrebbe lontani dal vero se si rimarcasse che un altro dei grands arrêts del diritto sindacale repubblicano - la sentenza n. 334 del 1988 della Corte costituzionale, sul sindacalismo dei quadrii $^{27}$ - si è mosso su un continuo di ambiguità terminologiche e sostanziali: esito forse inevitabile, in una vicenda in cui si contrapponeva, ai fini dell'accesso alle prerogative del titolo III dello statuto dei lavoratori, il modello di sindacato confederale storico pluricategoriale o multicategoriale (che aggrega più categorie di prestatori di lavoro ed è operativo con proprie federazioni di categoria nella gran parte dei rami dell'economia) e un soggetto che aggrega un'unica categoria di prestatori di lavoro (i quadri) ed è perciò definibile monocategoriale, ma che al contempo ha una struttura organizzativa che si potrebbe dire a sua volta multicategoriale, in quanto radicata in una pluralità di rami dell'economia ${ }^{28}$.

In breve, cosa si debba intendere con il termine categoria, quan-

26 Cfr. G. Suppiej, Fonti per lo studio del diritto sindacale, Padova, Cedam, 1965, pp. 120-121; più di recente, G. Orlandini, Conflitto collettivo, in Enc. Dir., Annali, IX, 2016, p. 112.

27 C. cost. 24 marzo 1988, n. 334, in Giur. Cost., 1988, II, p. 1433, nota M. Dell'Olio, Mass. Giur. Lav., 1988, p. 189, nota R. Pessi, Riv. It. Dir. Lav., 1988, II, p. 549, nota V.A. Poso.

28 Dietro le ambiguità terminologiche si celavano delicate questioni di politica sindacale attinenti alle linee organizzative dei soggetti storici: ad essere in discussione era, cioè, la nozione tradizionale di categoria professionale: v. M. GRANDI, Il problema della maggiore rappresentatività sindacale davanti alla Corte costituzionale (nella questione dei Sinquadri), in Riv. It. Dir. Lav., 1989, I, p. 141 ss., F. LUNARDON, Rappresentatività sindacale e categorie professionali, in La rappresentatività del sindacato, Quaderni di diritto del lavoro e delle relazioni industriali, Torino, Utet, 1989, p. 75 ss. 
do è adoperato nel diritto positivo, dalla giurisprudenza o dalle stesse parti sociali, non è sempre agevole da stabilire ${ }^{29}$. «Quando ad una parola vengono richiesti servizi così diversi, complessi e casuali, da molteplici agenti e per molteplici fini, può risultare incapace di selezioni fino a diventare mera evocazione» ${ }^{30}$ : è un tema, quello che ci occupa, nel quale il continuo intrecciarsi di diversi piani di linguaggio - quello del diritto e dei suoi interpreti e quello dei rapporti sindacali, ciascuno con una propria logica formale e sostanziale ${ }^{31}$ - complica non poco i tentativi di operare una ricostruzione tecnico-giuridica. Per avvicinarsi ai temi oggetto di questo studio, è necessario, perciò, spostare gradualmente l'attenzione verso le questioni specifiche che si pongono in materia di contrattazione collettiva, in modo da dotare il vocabolo di una migliore portata selettiva, che lo renda utilizzabile nella ricerca ${ }^{32}$.

\section{Categoria professionale e categoria sindacale}

Saggiandone una prima declinazione volta ad un utilizzo in concreto, il termine categoria può designare una serie aperta di soggetti, che condividono una collocazione nell'economia e nel mercato del

29 Cfr. P. Tosi, Concorrenza, lavoro, diritti (aspetti collettivo-sindacali), in Arg. Dir. Lav., 2017, p. 1350, ma v. spec. nota 34.

30 Il passo è tratto da M. Pedrazzoli, Alle origini del dibattito giuridico-istituzionale sulla democrazia industriale: il caso italiano, in AA.VV., Studi in onore di Tito Carnacini, I, Studi di diritto costituzionale, diritto civile, diritto del lavoro, diritto commerciale, Milano, Giuffrè, 1983, p. 796: le osservazioni che l'A. formula a proposito dell'espressione "democrazia industriale" sembrano, per così dire, ben rappresentare anche i profili di ambiguità terminologiche e di sostanza che riguardano il vocabolo "categoria". Del medesimo A., per una più approfondita digressione semantica sulla democrazia industriale, v. Democrazia industriale e subordinazione. Poteri e fattispecie nel sistema giuridico del lavoro, Milano, Giuffrè, 1985, p. 133 ss.

31 Cfr. F. Scarpeldi, Il Testo Unico sulla rappresentanza tra relazioni industriali e diritto, in Dir. Rel. Ind., 2014, p. 688.

32 Traccia, in poche ma significative pagine, queste linee metodologiche, volte a indirizzare il significato delle nozioni giuridiche verso gli «scopi per cui vengono formulate» e verso le «mutevoli situazioni storico-culturali nelle quali vengono proposte ed usate», L. Bagolini, Mistero ed esperienza sociale e giuridica, in Riv. Trim. Dir. Proc. Civ., 1961, p. 949 (per la citazione); v. altresì, alcuni anni prima, G. CALogero, La polemica sui concetti giuridici, in Riv. Dir. Comm., 1945, I, p. 112 ss. e A.C. Jemolo, Ancora sui concetti giuridici, ivi, p. 130 ss. 
lavoro, id est un raggruppamento professionale, identificabile sulla base di elementi convenzionalmente stabiliti ${ }^{33}$.

Ad esempio, mediante le attività di raccolta e di classificazione di dati sulle forze produttive e del lavoro in un dato perimetro, gli istituti di analisi statistica forniscono periodicamente tassonomie delle occupazioni, utilizzate a fini di divulgazione e di studio, ma anche dall'attore pubblico per predisporre misure di intervento nel mercato del lavoro ${ }^{34}$. Le categorie professionali sono presupposte o identificate all'occasione dal legislatore, per individuare l'ambito di applicazione di disposizioni di legge o di altri provvedimenti. Al costo di una marcata semplificazione, può osservarsi come il legislatore sia libero di individuare le categorie che sono assoggettate a talune discipline protettive ${ }^{35}$, ossia i destinatari delle norme ${ }^{36}$.

Tuttavia, tali partizioni non dovrebbero avere effetto, se non ai fini dell'applicazione delle medesime disposizioni ${ }^{37}$. Ciò è vero specialmente sul terreno dei rapporti sindacali, ove i termini del ragionamento sono per così dire invertiti. Con l'operare dell'art. 39, c. 1, Cost., assume rilievo predominante un elemento volitivo del soggetto storico. Il concetto di categoria è definito dall'attributo "sindacale" (se si preferisce, categoria in senso organizzativo). Il vocabolo non perde il significato elementare di selezione di un segmento della realtà socioeconomica, ma si qualifica in modo dirimente per l'autodeterminazione dei confini di quest'ultimo ${ }^{38}$.

33 Si tratta di un significato elementare della parola, avverte F. Liso, Categorie e qualifiche del lavoratore, in Enc. Giur. Trecc., VI, 1988, p. 1. V. altresì V. Simi, Formazioni sociali e categoria professionale, in Riv. Trim. Dir. Proc. Civ., 1965, p. 436 ss.

34 Ad esempio, Istat, La classificazione delle professioni, Roma, Istituto nazionale di statistica, 2013.

35 A. E. Cammarata, Il significato e la funzione del «fatto» nell'esperienza giuridica (1929), in ID. Formalismo e sapere giuridico. Studi, Milano, Giuffrè, 1963, pp. 285-287. Più di recente, R. SAcco, Fatto giuridico, in Dig. Civ., Aggiornamento, 2010, p. 616.

36 Invero, la questione dell'individuazione dei destinatari delle norme di legge è più complessa della soluzione oltremodo lineare alla quale si allude con la considerazione di cui al testo. Non sembra tuttavia necessario approfondirne i termini, per l'attinenza limitata con il tema di questa ricerca: cfr., comunque, F. Modugno, Norma giuridica (teoria generale), in Enc. Dir., XXVIII, 1978, p. 337, spec. note 35-38.

37 C. Balducci, Il sindacato, I, L'organizzazione sindacale, Torino, Utet, 1984, p. 87, G. Prola, L'inquadramento dei datori di lavoro, Milano, Giuffrè, 1993, passim. 38 F. Liso, Categorie e qualifiche del lavoratore, cit., pp. 1-2. 
La categoria sindacale, pur essendo naturaliter individuata per rinvio ad elementi in grado di suggerire una collocazione nel mondo del lavoro e dell'economia (ad esempio, «le lavoratrici e i lavoratori operanti nell'impresa metalmeccanica italiana»), altro non è se non una proiezione identitaria del gruppo, che si determina, appunto, da sé.

Non vi è, perciò, coincidenza (per lo meno, non per principio) tra le categorie sindacali e gli aggregati professionali che si formano nel mondo del lavoro o per come sono identificati dagli istituti di statistica o dal legislatore. Come dire che non è la rassomiglianza tra le attività alle quali i lavoratori sono addetti, o un qualche tipo di determinismo socioeconomico ${ }^{39}$, a identificare un interesse collettivo, bensì il fatto che, in autonomia, un gruppo - più o meno ampio e più o meno coeso al proprio interno - si riconosca in un'esperienza da rappresentare ad altri, individui, gruppi e istituzioni $^{40}$, diventando, per via di un momento organizzativo, soggetto storico $^{41}$. Al proposito non dovrebbero più esserci voci discordan$\mathrm{ti}^{42}$. Può ritenersi superata, cioè, ogni nozione ontologica di interesse collettivo, precostituita dalla composizione o da una struttura bloccata dei gruppi professionali, che il sindacato si limiterebbe a riconoscere e fare propria ${ }^{43}$. Un interesse della categoria è concepibile come momento ancora «teorico», che nell'organizzazione è in grado di reificarsi ${ }^{44}$; il che non potrebbe avvenire senza una determinazione politico-identitaria, in un andamento che pone al centro il soggetto e la sua azione.

39 J.-F. PoIsson, Rapport sur la négociation collective et les branches professionnelles, Rapport au Premier ministre, Paris, La documentation française, Remis le 28 avril 2009, p. 9.

40 F. Santoro-Passarelli, Autonomia collettiva, giurisdizione, diritto di sciopero (1949) e L'autonomia dei privati nel diritto dell'economia (1956), in ID., Saggi di diritto civile, Napoli, Jovene, 1961, pp. 177 ss., 227 ss.

41 G. Giugni, Libertà sindacale, in Dig. Comm., IX, 1993, pp. 26-27.

42 La posizione di cui al testo si può fare risalire a G. GiUgNI, La funzione giuridica del contratto collettivo di lavoro, in Il contratto collettivo di lavoro, Atti del III congresso nazionale dell'Aidlass, Milano, Giuffrè, 1968, p. 30 ss. e a M. PERsiANI, Saggio sull'autonomia privata collettiva, Padova, Cedam, 1972, pp. 33-34, nota 106, pp. 69-70, 74.

43 B. CARuso, Rappresentanza sindacale e consenso, Milano, Franco Angeli, 1992, p. 111 ss.

44 M.G. Garofalo, Interessi collettivi e comportamento antisindacale dell'imprenditore, Napoli, Jovene, 1979, p. 156. 
Per quanto la forma di aggregazione sindacale più diffusa nel sistema italiano di relazioni industriali sia quella delle federazioni di categoria, non vi è alcuna inferenza necessaria tra le categorie sindacali e, per semplificare, il complesso degli addetti ad un medesimo ramo dell'industria o branca produttiva o settore merceologico ${ }^{45}$. Si pensi alle aggregazioni per mestiere o per professione, la cui diffusione è aumentata, con l'articolazione del mercato del lavoro, dagli anni Ottanta del secolo scorso in avanti ${ }^{46}$; all'eventualità che sindacati attivi in un'impresa sovranazionale diano vita ad organizzazioni a misura di azienda, svincolate dalle federazioni del settore e dalle loro confederazioni ${ }^{47}$; ancora, ai tentativi di costituire sindacati intercategoriali, per superare, in alcune catene del valore (si pensi alla logistica $)^{48}$, il modello tradizionale della struttura sindacale cd. verticale, che sarebbe inadatto a fronteggiare le linee più intensive dell'odierno sviluppo dell'economia di mercato ${ }^{49}$; infine, alle forme particolarmente vivaci di sindacalismo che sta sollecitando l'evoluzione in senso digitale dell'economia ${ }^{50}$.

Ciascun gruppo, con una scelta insindacabile, individua un proprio ambito di inquadramento, che può sovrapporsi, in tutto o in

45 F. ScARPElli, Le fonti e il contenuto del principio di libertà sindacale, in C. Zoli (a cura di), Le fonti. Il diritto sindacale, in F. CARINCI (dir. da), Diritto del lavoro. Commentario, II ed., Torino, Utet, 2007, p. 75.

46 La genesi degli svolgimenti attuali risale, come noto, agli anni Settanta e Ottanta del secolo scorso, ma la situazione è cambiata rispetto al quadro degli «extra-confederali» allora descritto da A. Accornero, La parabola del sindacato, Bologna, Il Mulino, 1992, p. 259 ss., o da G. Della Rocca, Il sindacato, in G.P. Cella, T. TReu, Le nuove relazioni industriali. L'esperienza italiana nella prospettiva europea, Bologna, Il Mulino, 1998, p. 103.

47 Un cenno, prendendo spunto dal "caso Fiat", in R. SAlomone, Verso un nuovo regime collettivo per l'auto?, in F. CARINCI (a cura di), Da Pomigliano a Mirafiori: la cronaca si fa storia, Milano, Ipsoa, 2012, p. 107 ss., nonché in F. Siotтo, La categoria come ambito di applicazione e perimetro di misurazione della rappresentanza, in Riv. It. Dir. Lav., 2017, I, pp. 328-329, nota 62.

48 A. Allamprese, O. Bonardi (a cura di), Logistica e lavoro, Quaderni Riv. Giur. Lav., Roma, Ediesse, 2018, Se. Bologna, S. CuRI, Relazioni industriali e servizi di logistica: uno studio preliminare, in Dir. Lav. Rel. Ind., 2019, p. 125 ss.

49 Del tema ci si occupava già negli anni Novanta: A. Tursi, Sindacati e Cobas: il contratto collettivo "conteso", in Riv. It. Dir. Lav., 1990, I, p. 318 ss.

50 P. TulLINI, L'economia digitale alla prova dell'interesse collettivo, in Lab. Law. Iss., 2018, 1, p. 15 ss. 
parte, con quello degli altri ${ }^{51}$. Si è scritto che «Dalla libertà di ciascun gruppo di organizzarsi nel medesimo ambito di riferimento, o di individuare una categoria professionale trasversale a quella identificata da altri sindacati, deriva anche la garanzia del pluralismo sindacale» ${ }^{52}$.

In effetti, è indubbio che l'ordinamento giuridico garantisca la libertà, non solo di dar vita a sindacati, ma anche, per questi, di sviluppare una diramazione organizzativa e di delineare, per lo più mediante uno statuto, un ambito nel quale esercitare le attività di reclutamento, protezione e promozione degli interessi di lavoro. In tale sfera di autonomia è precluso allo Stato di interferire con la scelta dell'organizzazione di «estendersi fin dove vuole» e di raggruppare i lavoratori secondo criteri considerati opportuni, affinché l'organizzazione «nel suo insieme ed in ogni sua parte, nel suo "se" e nel suo "come" proceda da libertà e sia indipendente da imposizioni autoritarie» ${ }^{53}$. Per il soggetto collettivo è questione di «determinazione della propria identità [la quale] non può non restare assoluta, siccome [...] costituente profilo o elemento essenziale, e se si vuole radice o incrocio di altri, in ogni explicatio della formula di garanzia» $^{54}$.

L'espressione «L'organizzazione sindacale è libera» (art. 39, c. 1, Cost.) ha acquisito questo significato, «nei confronti della sfera soggettiva del sindacato» ${ }^{55}$, in virtù di una sistemazione della giurisprudenza costituzionale che potrebbe considerarsi acquisita già alla

51 M. Persiani, La retribuzione tra legge, autonomia collettiva e determinazione giudiziale, in AA.VV., Nuove forme di retribuzione e attualità dei principi costituzionali. Atti dell'incontro di studio Roma, 3 febbraio 1997, Quaderni Arg. Dir. Lav., Padova, Cedam, 1998, p. 39.

52 P. Bellocchi, La libertà sindacale, in G. Proia (a cura di), Organizzazione sindacale e contrattazione collettiva, in F. CARINCI, M. PERSIANI (dir. da), Trattato di diritto del lavoro, Padova, Cedam, 2014, p. 38.

53 C. Esposito, Lo Stato e $i$ sindacati nella Costituzione italiana, in ID., La Costituzione italiana. Saggi, Padova, Cedam, 1954, p. 160.

54 M. Dell'Olio, L'organizzazione e l'azione sindacale, Padova, Cedam, 1980, p. 136.

${ }_{55}$ Gio. Branca, L'associazione sindacale, Milano, Giuffrè, 1960, pp. 102103. 
fine degli anni Sessanta del secolo scorso ${ }^{56}$. Furono delineati all'epoca alcuni dei capisaldi del nuovo ordinamento sindacale democratico, anche per opposizione al precedente regime corporativo ${ }^{57}$. Secondo quanto osservato dalla Corte costituzionale, le categorie professionali non derivano da «astratti concetti classificatori delle attività produttive», ma «dalla spontanea organizzazione sindacale» ${ }^{58}$. È da un atto di volontà che traggono fondamento tali entità della realtà socioeconomica ${ }^{59}$, la cui sfera di elezione è sottratta ad un intervento diretto della legge ${ }^{60}$, atteso che la «libertà di organizzazione e di inquadramento» non potrebbe essere «limitata o annullata», da parte dei pubblici poteri, senza compromettere altresì una delle basi dell'ordine democratico ${ }^{61}$.

In breve, il diritto di definire arbitrariamente una sfera di inquadramento elettivo è un corollario immediato del principio di libertà sindacale $^{62}$ : è questa anzi la manifestazione «più classica della liber-

56 Cfr. M. Ricci, Autonomia collettiva e giustizia costituzionale, Bari, Cacucci, 1999, pp. 304-309.

57 Dopo averne preparato il campo per alcuni anni (A. Aquarone, Aspirazioni tecnocratiche del primo fascismo, in Nord e Sud, 1964, 9, p. 117 ss.), il regime aveva realizzato d'imperio una razionalizzazione del sistema di relazioni industriali, mediante la riconduzione delle forze dell'economia e del lavoro entro un numero predeterminato di categorie, sulla base di criteri di inquadramento fissati $e x$ ante, ma soggetti nel tempo a revisioni ed aggiustamenti (R. RICHARD, Lineamenti generali dell'attività di inquadramento sindacale, Pavia, Libreria internazionale Garzanti, 1942). In relazione a ciascuna categoria era attribuita la rappresentanza legale ad un'associazione soltanto dei datori di lavoro e dei lavoratori, in modo da evitare o, comunque, ridurre entro termini accettabili, l'eventualità che si sovrapponessero le aree di influenza organizzativa e di competenza negoziale tra le associazioni (cfr., per un quadro riassuntivo, L. Riva SANSEVERINo, Corporazione (diritto corporativo), in Enc. Dir., X, 1962, p. 679 ss.).

58 C. cost. n. 70/1963, cit.

59 C. cost. 26 giugno 1969, n. 105, in Giur. Cost., 1969, p. 1597, nota R. Flammia, Mass. Giur. Lav., 1969, p. 383, nota D. Guerrieri.

60 C. cost. 26 gennaio 1960, n. 1, in Giur. Cost., 1960, p. 10, nota V. SicA, che respinse la censura di illegittimità costituzionale della 1. 22 dicembre 1956, n. 1589, sul distacco da Confindustria delle aziende a prevalente partecipazione statale, con l'argomentazione che l'art. 3 della legge si sarebbe limitato a creare il presupposto di fatto per il raggiungimento dello scopo voluto dal legislatore storico.

61 C. cost. 22 dicembre 1965, n. 88, in Riv. Giur. Lav., 1966, II, p. 179, nota G. Pera, Riv. Dir. Lav., 1966, II, p. 17, nota G. Zangari.

62 U. Prosperetti, Libertà sindacale (premesse generali), in Enc. Dir., XXIV, 1974, p. 494 ss. In chiave storico-giuridica, G. MAzzonI, La conquista della libertà sindacale, Roma, Edizioni Leonardo, 1947, p. 192, ma passim. V. altresì su que- 
tà organizzativa ${ }^{63}$. Dal momento che la scelta di fondo dell'ordinamento giuridico non è stata di tipizzare una certa forma di coalizione, bensì di garantire i dispositivi socioeconomici volti ad un'effettiva tutela degli interessi di lavoro ${ }^{64}$, è parso irrinunciabile che le organizzazioni sindacali fossero a questi fini munite di autodeterminazione ${ }^{65}$. Solo in un momento successivo, a giochi fatti, se si vuole, dal punto di vista organizzativo, il diritto positivo interviene sui rapporti sindacali, dando per acquisita la configurazione del soggetto ${ }^{66}$. Nel complesso, ciò si risolve in una situazione di base della collocazione dei sindacati nell'ordinamento statuale ${ }^{67}$.

sti temi F. Galgano, Delle associazioni non riconosciute e dei comitati. Art. 3642, Bologna-Roma, Zanichelli-Società editrice del Foro italiano, 1967, pp. 53-55. Una nutrita serie di disposizioni di diritto sovranazionale, vincolanti per lo Stato italiano, nel sancire il principio di libertà sindacale, tutelano parimenti e, in qualche modo, ulteriormente rafforzano, il diritto di definire in autonomia una struttura organizzativa. Per un inquadramento complessivo del tema, cfr. A. BaYlos GraU, L. Zoppoli (a cura di), La libertà sindacale nel mondo: nuovi profili e vecchi problemi. In memoria di Giulio Regeni, Quaderni Dir. Lav. Merc., Napoli, Editoriale Scientifica, 2019, sez. I.

63 Così, B. Veneziani, Stato e autonomia collettiva. Diritto sindacale comparato, Bari, Cacucci, 1992, p. 75.

64 R. Flammia, Contributo all'analisi dei sindacati di fatto. I. Autotutela degli interessi di lavoro, Milano, Giuffrè, 1963.

65 E. Ghera, Libertà sindacale e ordinamento della polizia, in Giur. Cost., 1976, p. 656 ss.

66 M. Napoli, Sindacato, in Dig. Comm., XVI, Appendice, 1999, p. 510.

67 M.V. Ballestrero, Interesse collettivo e conflitto, in Lav. Dir., 2018, p. 426. Come noto, la libertà di inquadramento incontra penetranti limitazioni con riferimento ad alcune situazioni che sono state oggetto di dibattito anche di recente, ma che toccano appena il tema vero e proprio oggetto di questa ricerca. È il tema della libertà sindacale e di inquadramento degli appartenenti alle forze di polizia ed alle forze armate. Per le forze di polizia, sin dal 1981 (legge n. 121) è stato riconosciuto un diritto di libertà sindacale cd. separata, che consente la costituzione di associazioni sindacali tra il personale delle forze di polizia, a condizione che queste non siano inquadrate nelle organizzazioni confederali dei lavoratori (cfr. M. NICOLosi, Libertà sindacale separata e Polizia di Stato, in Lav. Giur., 2012, p. 1051 ss.). Un recente intervento della Corte costituzionale ha affermato un principio similare per le forze armate, aprendo alla costituzione di associazioni «professionali a carattere sindacale alle condizioni e con i limiti fissati dalla legge», purché non aderiscano «ad altre associazioni sindacali»: C. Cost. 13 giugno 2018, n. 120, in Dir. Lav. Merc., 2018, II, p. 621, nota P. Lambertucci, M.D. Ferrara, Dir. Rel. Ind., 2018, p. 1185, nota M. Falsone, Riv. Giur. Lav., 2018, II, p. 611, nota M. Forlivesi, Lav. Pubb. Amm., 2018, 4, p. 75, nota L. M. Dentici; v. altresì P. Lambertucci, Le linee 


\section{Categoria contrattuale}

Secondo terminologia in uso nel diritto sindacale, è categoria anche quella "contrattuale". Tale espressione allude al campo di applicazione dei contratti collettivi. Di analogo significato sono formule quali "unità contrattuale", "ambito contrattuale", "area negoziale", "area contrattuale" o simili combinazioni ${ }^{68}$. Anche l'espressione inglese "bargaining unit" è utilizzata correntemente dalla dottrina italiana, sin dagli anni Cinquanta del secolo scorso ${ }^{69}$. Tali formule saranno adoperate nella ricerca in modo interscambiabile. L'espressione "comparto di contrattazione" è anch'essa, in sostanza, sinonimo di categoria contrattuale, ma il suo utilizzo è proprio specialmente del settore pubblico.

In questa materia è frequente anche l'uso dei termini "giurisdizione" (nella letteratura scientifica) ${ }^{70}$ e "competenza" (negli accordi

evolutive della libertà di associazione sindacale per i militari, in Lav. Dir., 2019, p. 305 ss.

68 V. ad esempio il Protocollo d'intesa 31 maggio 2013 concluso tra Confindustria e Cgil, Cisl e Uil, nel quale le espressioni "ambito di applicazione del contratto collettivo" (sub «Misurazione della rappresentatività», n. 2 e sub «Titolarità ed efficacia della contrattazione», n. 1) e "ambito contrattuale" (sub «Misurazione della rappresentatività», n. 4) ricorrono, in sostanza, in modo interscambiabile.

69 Sembra a chi scrive che ciò si possa spiegare per la profonda influenza esercitata dall'esperienza americana e anglosassone di relazioni industriali su alcuni celebri giuristi italiani che, nel dopoguerra, parteciparono da protagonisti al rinnovamento degli studi sul tema della categoria (si allude specialmente a Gino Giugni e Federico Mancini). Per non fare che alcuni esempi, tratti dalla recente letteratura di diritto sindacale, l'espressione bargaining unit è utilizzata in P. CAMPANELla, Rappresentatività sindacale: fattispecie ed effetti, Milano, Giuffrè, 2000, p. 156, A. VIscomi, Soggettività sindacale e categorie contrattuali, in Riv. Giur. Lav., 2014, I, p. 82, V. PAPA, Verso una rappresentanza misurata? Strumenti e metodi di misurazione della rappresentatività datoriale, cit., p. 25.

70 L'uso dell'espressione "giurisdizione", in riferimento alle sfere di influenza organizzativa e negoziale delle organizzazioni sindacali, divenne corrente negli studi di diritto sindacale a partire dagli anni Cinquanta e Sessanta del secolo scorso, dopo essere stata mutuata dall'esperienza americana e anglosassone (v. nota prec.): cfr. G.F. MANcini, La rappresentanza sindacale nel diritto statunitense e l'art. 39 della Costituzione italiana, in Riv. Dir. Lav., 1950, I, spec. pp. 472-474, G. GIUGNI, Bargaining units and labor organization in Italy, in Ind. Lab. Rel. Rev., 1957, 10, 3, p. 424 ss.; nella dottrina anglosassone, su questi temi, il notissimo O. Kahn-Freund, Intergroup Conflicts and Their Settlement, in Brit. J. Soc., 1954, 5, 3, spec. p. 199. 
tra le parti sociali) ${ }^{71}$. Più che al campo di applicazione dei contratti collettivi, si allude in questo modo ad una sfera di influenza o, se si vuole, di autorità, che le organizzazioni sindacali rivendicano per un dato segmento del mondo dell'economia e del lavoro e che è loro riconosciuta (o meno, o non interamente) all'interno delle relazioni industriali, sotto il profilo organizzativo e della contrattazione collettiva.

È significativo che nella prassi contrattuale sia di recente emersa (o semplicemente divenuta di uso più comune) una nuova espressione: "perimetri" contrattuali (o negoziali). Il suo utilizzo ricorre più volte nell'accordo interconfederale del 9 marzo 2018 stipulato tra Confindustria e Cgil, Cisl e Uil su «Contenuti e indirizzi delle relazioni industriali e della contrattazione collettiva» (cd. Patto per la fabbrica $)^{72}$. Dopo l'accordo, tale espressione è rapidamente divenuta corrente nella pubblicistica di settore ${ }^{73}$.

A ben vedere, essa è segno dei tempi: benché non sia che un altro equivalente della più tradizionale formula categoria contrattua$1 \mathrm{e}^{74}$, non vi è dubbio che il suo utilizzo si ricolleghi alla contesa, in

71 V. ad esempio l'Accordo interconfederale 26 novembre 2015 concluso tra Confcommercio - Imprese per l'Italia e Cgil, Cisl e Uil, lett. $A$ ) «Contrattazione collettiva nazionale di categoria», n. 3 «Modalità di rilevazione della rappresentatività», punto III (ove le parti stipulanti alludono in particolare alla competenza negoziale di ciascuna organizzazione in relazione alla misurazione della rappresentatività ai fini della contrattazione nazionale).

72 V., nell'accordo citato nel testo, sub n. 4 «Democrazia e misura della rappresentanza», passim.

73 Cfr. M. Marazza, Perimetri e rappresentanze sindacali (dei datori di lavoro e dei lavoratori), in Lab. Law Iss., 2018, 2, p. III ss., F. Liso, Qualche erratica considerazione sul recente accordo interconfederale Confindustria, Cgil, Cisl e Uil del 9 marzo 2018, in Boll. Adapt, 23 aprile 2018, n. 16, M. Ricci, L'accordo interconfederale del 9 marzo 2018: una svolta dagli esiti incerti, in Arg. Dir. Lav., 2018, p. 1412 ma passim, L. Bordogna, L'accordo Confindustria-sindacati del 9 marzo 2018 su relazioni industriali e contrattazione collettiva, in Dir. Lav. Rel. Ind., 2019, pp. 46-47. L'espressione "perimetri contrattuali” era talvolta utilizzata anche prima del Patto per la fabbrica: v. M. MARAzZA, Dalla "autoregolamentazione" alla "legge sindacale"? La questione dell'ambito di misurazione della rappresentatività sindacale, in Arg. Dir. Lav., 2014, p. 609.

74 P. ICHINo, La crepa interna dell'articolo 39 della Costituzione, in www.pietroichino.it, il quale, dopo aver rimarcato come le espressioni "categorie" e "perimetri" siano equivalenti, avverte che tra le due utilizzerà la seconda, proprio «per adottare la terminologia del Patto per la fabbrica» (corsivo dell'autore). Contra, M. 
atto nelle relazioni industriali di questi anni, su quali organizzazioni siano competenti a (o abbiano giurisdizione per) stipulare i contratti collettivi per le diverse tipologie di attività economica. In altre parole, l'utilizzo dell'espressione perimetri contrattuali è indicativo di una situazione di tensione circa i confini dei contratti collettivi di categoria: situazione che, a sua volta, deriva dalla maggiore frequenza con la quale, rispetto al passato, si verificano commistioni tra le sfere di influenza delle organizzazioni di interessi, specialmente dal lato datoriale ${ }^{75}$. Il Patto per la fabbrica non lascia dubbi, a questo proposito: l'operazione di «ricognizione dei perimetri della contrattazione collettiva nazionale di categoria» e di individuazione dei soggetti più rappresentativi in tali ambiti, che esso preconizza (n. 4, lett. $a-b$ ), è volta ad assicurare «il rispetto dei perimetri della contrattazione collettiva e dei suoi contenuti» e ad impedire che «soggetti privi di adeguato livello di rappresentatività» possano (meglio: continuino a) «violare o forzare arbitrariamente i perimetri e gli ambiti di applicazione dei contratti collettivi nazionali di categoria» (corsivi miei).

Tanto chiarito, è appena il caso di precisare che non vi è corrispondenza (per lo meno, non necessariamente o per principio) tra le categorie sindacali e le categorie contrattuali ${ }^{76}$. Situazione ricorrente, per il sistema italiano di relazioni industriali, è che un'organizzazione sindacale $\mathrm{o}$, più frequentemente, una coalizione di esse, concluda più d'un contratto collettivo, ossia contribuisca, per così dire, a "creare" più d'una categoria contrattuale. Tuttavia, sarebbe inesatto per ciò solo affermare che la categoria sindacale sia più ampia di quella contrattuale, che nella prima risulterebbe - come in un sottoinsieme - ri-

Marazza, Perimetri e rappresentanze sindacali (dei datori di lavoro e dei lavoratori), cit.

75 V. Leccese, G. Roma, M. Lozito, La contrattazione nazionale di categoria, in M. Mascini (a cura di), l'Annuario del lavoro 2019, Roma, Il diario del lavoro, 2019, pp. 67-71.

76 Il rilievo è già in Gio. BrancA, L'associazione sindacale, cit., pp. 103-104, «proprio per la diversa impostazione consentita dalla libertà sindacale», ed altresì in L. Mengoni, Il regime giuridico delle organizzazioni professionali in Italia, in G. BoldT et al., Il regime giuridico delle organizzazioni professionali nei Paesi membri della C.E.C.A., Lussemburgo, Comunità europea del carbone e dell'acciaio Alta autorità, 1966 , pp. 470-471, nota 76 . 
compresa. Ad essere diverse sono, ab imis, le sintesi che inducono le parti sociali ad individuare l'una e l'altra. Nel primo caso, tale sintesi è l'esito di un processo organizzativo-identitario interno al soggetto medesimo ${ }^{77}$. Nel secondo, è l'esito di un processo di contrattazione collettiva e, perciò, di un compromesso raggiunto, in una logica di opposizione $^{78}$, tra le organizzazioni dei lavoratori e dei datori di lavoro ${ }^{79}$.

Tali compromessi possono avere esiti molto diversi, in base all'articolazione della struttura contrattuale ${ }^{80}$.

Il campo di applicazione dei contratti collettivi può essere definito sulla base della tipologia di attività economica svolta dai datori di lavoro, come per lo più avviene nella contrattazione collettiva cd. di settore o per ramo d'industria, che aggrega segmenti del tessuto produttivo re-

77 Beninteso, per quanto "interna", l'operazione con la quale il soggetto collettivo individua il segmento della realtà socioeconomica nel quale intende operare è inevitabilmente influenzata dal più ampio contesto delle relazioni industriali. Si tenga presente, d'altra parte, come tali operazioni avvengano, almeno potenzialmente, a discapito di altri gruppi, che per esito di una decisione altrui potrebbero vedere circoscritta la propria sfera di reclutamento e di organizzazione. A questo proposito, è istruttiva l'esperienza delle relazioni industriali del Regno Unito, atteso che il TUC si è dotato, fin dai primi decenni del XX secolo, di un sistema articolato di delimitazione delle sfere di influenza sindacale, che include meccanismi di risoluzione di eventuali controversie. Si tratta dei cd. Bridlington principles, ai quali Otto Kahn-Freund alludeva nei termini di un «indispensable element of our system of industrial relations» (cfr. P. Davies, M. FreEdLand, Kahn-Freund's Labour and the law, London, Stevens, 1983, p. 235). B. CARuso, Per un intervento eteronomo sulla rappresentanza sindacale: se non ora quando!, in WP CSDLE “Massimo D'Antona”.IT, 206/2014, pp. 6-7, ma v. anche nota 10, si sofferma su questo aspetto del sistema inglese, in una riflessione sul tema che ci occupa. Anche T. TREU, Regole e procedure nelle relazioni industriali: retaggi storici e criticità da affrontare, in WP CSDLE “Massimo D'Antona”.IT, 396/2019, pp. 12-13, riprende, parimenti, sotto questo profilo l'esperienza inglese di relazioni industriali, paragonandovi altresì quella tedesca, ove il DGB ha adottato principi di ripartizione ordinata delle giurisdizioni organizzative e negoziali e di risoluzione di eventuali controversie. Per l'esperienza italiana, su questi profili, v. F. Siотто, Ordinamento sindacale democratico. La prospettiva interna, Torino, Giappichelli, 2018, p. 136 ss.

78 E. Gragnoli, Esiste ancora un ordinamento intersindacale?, in AA.VV., Studi in memoria di Mario Giovanni Garofalo, 1, Bari, Cacucci, 2015, p. 445.

79 M. Novella, Applicazione del contratto collettivo e categoria professionale. Vecchi e nuovi problemi, in Riv. Giur. Lav., 1999, I, p. 360 nota 11, ma passim.

80 Cfr. R. SANtucci, Sistema di relazioni industriali e contratto collettivo, in R. SANtucci, L. Zoppoli (a cura di), Contratto collettivo e disciplina dei rapporti di lavoro, II ed., Torino, Giappichelli, 2004, p. 11 ss., T. Treu, Contrattazione collettiva, in Enc. Dir., Annali, VI, 2011, p. 227 ss. 
lativamente omogenei. In altri ordinamenti vi si allude anche in termini di ambito di applicazione "funzionale" dei contratti collettivi ${ }^{81}$.

Nella ripartizione per unità dei sistemi contrattuali, un altro elemento al quale è data rilevanza consiste nella collocazione delle attività economiche in una certa area geografica, solitamente individuata con un collegamento ad una porzione più o meno ampia (fino all'intero, come per lo più avviene nell'esperienza italiana) del territorio nazionale: vi si può riferire in termini di campo di applicazione territoriale dei contratti collettivi.

Nulla impedisce, tuttavia, che sia un elemento ricollegabile ai lavoratori a costituire il tratto caratterizzante dell'unità negoziale. Ad esempio, la contrattazione collettiva potrebbe essere svolta in modo differenziato per operai e impiegati: benché nel sistema italiano di relazioni industriali ciò, nella sostanza, sia stato superato con il cd. inquadramento unico ${ }^{82}$, altrove questo può essere ancora il caso $^{83}$. Nel modello italiano sono per lo più i dirigenti ad avere ottenuto dalle controparti il riconoscimento di unità negoziali ad hoc, benché in alcuni settori del mercato gruppi professionali dotati di particolare "leva" contrattuale abbiano ottenuto la medesima prerogativa.

Possono essere, inoltre, più o meno diffuse le unità negoziali definite sulla realtà d'impresa ${ }^{84}$, di gruppo ${ }^{85}$, di sito, di filiera, di di-

81 Ad esempio, per la Spagna, J.C. Villalon, Compendio de derecho del trabajo, XI ed., Madrid, Tecnos, 2018, p. 545; per il Belgio, R. Blanpain, Labour law in Belgium, IV ed., Alphen aan den Rijn, Kluwer law international, 2012, p. 367 ss.

82 Cfr. A. Garilli, Categorie dei lavoratori (in generale), in Dig. Comm., III, 1988, p. 40 ss.

83 Ad esempio, in Belgio, benché da alcuni anni sia emersa la volontà di superare gradualmente, con una revisione delle unità negoziali, questa caratteristica della contrattazione collettiva: un cenno in K. VANDAELE, Belgium: stability on the surface, mounting tensions beneath, in T. Müller, K. VANDAELE, J. WadDingTON, Collective bargaining in Europe: towards an endgame, I, Brussels, Etui, 2019, p. 55. È noto che nel sistema statunitense la definizione delle bargaining units si determina, in larga parte, per l'esistenza di una comunanza di interessi tra i lavoratori che dipende dall'attività da essi svolta o da loro requisiti o status professionali (per una recente rassegna degli orientamenti del NLRB in materia di definizione delle unità negoziali, v. Si. Bologna, La contrattazione collettiva negli Stati Uniti dal Wagner act ai nostri giorni, in Riv. It. Dir. Lav., 2016, I, p. 421 ss.).

84 U. Romagnol, Il contratto collettivo d'impresa, cit.

85 F. Lunardon, Autonomia collettiva e gruppi di imprese, Torino, Giappichelli, 1996, p. 195 ss., A. LASSANDARI, Il contratto collettivo aziendale e decentrato, Milano, Giuffrè, 2001, pp. 362-366. 
stretto, di rete e via discorrendo ${ }^{86}$ : invero, l'apertura di nuove unità negoziali è un processo che «in ragione soprattutto delle modifiche di una "geografia" industriale soggetta a rapide evoluzioni tecnologiche $[\ldots]$ non potrà forse mai esaurirsi né pervenire a risultati praticamente definitivi» ${ }^{87}$.

Non sfuggirà, peraltro, che per ragioni legate allo sviluppo del movimento sindacale ${ }^{88}$ e ad una risalente propensione della cultura giuridica ${ }^{89}$, nel gergo sindacale si identifica "la" categoria con le unità negoziali definite sulla base del settore produttivo di beni o servizi (il Ccnl): è il «contratto per eccellenza» ${ }^{90}$ che, non per caso, continua ad essere comunemente definito "di" categoria, quasi che questa sia la dimensione «aurea» $\mathrm{O}$ «naturale» di svolgimento delle attività sindacali ${ }^{91}$. Quanto ciò corrisponda oggi alla realtà dei processi economici e produttivi ed agli stessi orientamenti dei corpi intermedi, è questione tanto affascinante quanto sfuggente ${ }^{92}$, ma della quale la ricerca non si occuperà. Il tema vero e proprio oggetto dello studio sarà identificato e precisato nei prossimi paragrafi.

86 V. Bavaro, S. Laforgia, Contrattazione collettiva e "prossimità delle imprese". La struttura del contratto collettivo di filiera, distretto, rete d'imprese, in M.T. CARINCI (a cura di), Dall'impresa a rete alle reti d'impresa. Scelte organizzative e diritto del lavoro, Milano, Giuffrè, 2015, p. 107 ss., G. ZiLIo Grandi, La contrattazione collettiva nelle reti di imprese: spunti per una efficace implementazione, in Giur. It., 2017, p. 1482 ss.

87 Così scriveva, nel 1963, U. Romagnoli, Il contratto collettivo d'impresa, cit., p. 43.

88 In Italia, come nell'Europa continentale: P. MArginson, Industrial Relations at European Sector Level: The Weak Link?, in Eco. Ind. Dem., 2005, 4, p. 511 ss.

89 Non perdono di attualità le riflessioni di U. Romagnoli, Politica e tecnica nella contrattazione collettiva, in Riv. Trim. Dir. Proc. Civ., 1962, p. 245 testo e nota 11.

90 G.P. Cella, T. Treu, Relazioni industriali e contrattazione collettiva, Bologna, Il Mulino, 2009, p. 115.

91 M. Dell'Olio, L'organizzazione e l'azione sindacale, cit., p. 134.

92 E. GRagnoli, La parabola del contratto collettivo nella società economica italiana, in Lav. Giur., 2013, p. 653 ss. 


\section{Le tipizzazioni dell'autonomia collettiva}

La definizione più generale del concetto di categoria come "classi di realtà” (v. supra, § 2) rimanda a un'esigenza basilare di ogni proposizione normativa: l'identificazione di un complesso di situazioni di fatto alle quali ricollegare il verificarsi di effetti giuridici. È un'esigenza che deriva dall'impossibilità stessa di fornire una valutazione di qualcosa senza che si abbia prima per essa un oggetto ${ }^{93}$. A tale oggetto si allude, in genere, nel diritto, con il termine "fattispecie": come serie, cioè, di ipotesi di fatti astrattamente previsti e ordinati in una classificazione, ai quali ricollegare (in un momento che rimane distinto nel ragionamento) un trattamento ${ }^{94} \mathrm{o}$, se si preferisce, uno statuto ${ }^{95}$. La qualificazione operata dalla norma attribuisce alle ipotesi di fatti da essa previsti una rilevanza giuridica ${ }^{96}$. Non serve addentrarsi nel tema: da una lettura, come quella suggerita, elementare e fin troppo lineare ${ }^{97}$, si può ricavare l'esigenza di massima di «edificare il linguaggio concettuale» mediante serie di classificazioni, in modo da «descrivere in modo ordinato i fenomeni giuridici» ${ }^{98}$.

È da simili spunti che una parte della dottrina lavoristica ha preso le mosse, per rimarcare come anche la contrattazione collettiva, per definire il trattamento economico e normativo dei lavoratori, abbia l'esigenza preliminare di delimitare l'ambito di applicazione delle situazioni regolande, mediante un rinvio a serie più o meno articolate di ipotesi di fatti, tratte dal mondo dell'economia e del lavoro. Si deve, in particolare, a un saggio del 1990 di Pedrazzoli ${ }^{99}$, di avere identificato, all'interno della funzione normativa del contratto collettivo, una subfunzione qualificatrice, come «operazione in cui si delimita l'ambito p. 932 ss.

93 A. Cataudella, Fattispecie, in Enc. Dir., XVI, 1967, p. 926 ss., ma spec.

94 E. BetTI, Teoria generale del negozio giuridico, II ed., III rist., Torino, Utet, 1960, p. 2.

95 V. Buonocore, Impresa (diritto privato), in Enc. Dir., Annali, I, 2007, pp. 766-767.

96 E. BetTI, Teoria generale del negozio giuridico, cit., p. 3.

97 Dalla quale si dipartono riflessioni di respiro: R. SAcco, Fattispecie, in Dig. Civ., Agg., 2010, p. 594 ss.

98 Così, P.G. Monateri, Fattispecie, in Dig. Civ., VIII, 1992, p. 224.

99 M. Pedrazzoli, Qualificazioni dell'autonomia collettiva e procedimento applicativo del giudice, in Lav. Dir., 1990, pp. 355 ss., 549 ss. 
di incidenza della regola, ovvero si determinano le situazioni o "figure del fatto" ${ }^{100}$ «alle quali [tale regola] è imputata» ${ }^{101}$.

Come per qualsiasi altra regola di comportamento, l'esigenza di individuarne i destinatari mediante astratte tipizzazioni di situazioni di fatto è ineludibile: si tratta, avvertiva Ghezzi, di «clausole essenziali» dei contratti collettivi, non in applicazione delle disposizioni codicistiche sul contratto collettivo (spec. artt. 2069-2070), ma per via della «necessaria delimitazione degli effetti contrattuali [...] alla realtà e alle esigenze pratiche del fenomeno» ${ }^{102}$. L'esperienza comparata può confermare l'assunto. Da una parte, è significativo che, nei sistemi in cui la contrattazione collettiva è regolata dalla legge, le clausole sul campo di applicazione siano per lo più annoverate tra quelle da stipulare obbligatoriamente; peraltro, «in questa materia, l'inesistenza di una disposizione espressa non elimina l'obbligo per le parti di una previsione siffatta, atteso che da essa come è ovvio dipende la stessa applicabilità della norma» ${ }^{103}$. Dall'altra, non è meno rilevante che, in relazione ai sistemi autoregolati dalle parti sociali, si osservi comunemente come la definizione del campo di applicazione sia un indispensabile passaggio preliminare per la contrattazione collettiva ${ }^{104} \mathrm{o}$ uno dei suoi momenti più delicati ${ }^{105}$.

In breve, la previa delimitazione delle situazioni regolande me-

100 Ivi, pp. 377-378.

101 Ivi, p. 380.

102 G. GHEzzI, La responsabilità contrattuale delle associazioni sindacali, Milano, Giuffrè, 1963, p. 24, spec. nota 54. È un'ipotesi invero improbabile quella di un contratto collettivo concluso senza che sia in alcun modo specificato il campo di applicazione; si può prendere più realisticamente in considerazione l'ipotesi che le relative clausole non lo definiscano in modo inequivocabile: in tal caso, non è da escludere che si possa sopperire in via interpretativa, anche mediante altre clausole del contratto collettivo che orientino la scelta dell'interprete (Cass. 9 giugno 1993, n. 6412, in Riv. It. Dir. Lav., 1994, II, p. 291 ss., nota G.L. PINTO). Sul punto v. comunque il cap. IV, $\S 3$.

103 (Trad. mia dallo spagnolo). Cfr. J.C. Villalon (dir.), La negociación colectiva en Europa. Una perspectiva transversal, Madrid, Ministerio del Trabajo, Migraciones y Seguridad Social, Subdirección General de Información Administrativa y Publicaciones, 2019; ma spec. v. sub Contenido convencional (cap. redatto da R. G. Gordillo), pp. 435-436 (per la citazione testuale v. p. 435).

104 E. Cordova, Collective bargaining, in R. Blanpain (ed.), Comparative labour law and industrial relations, Dordrecht, Springen, 1982, pp. 229-230.

105 K. D. Ewing, J. Hendy, C. Jones (eds.), Rolling out the Manifesto for Labour Law, Liverpool, the Institute of Employment Rights, 2018, p. 20. 
diante contratto collettivo è un'esigenza logica prima che giuridica, come dimostra il fatto, in sé significativo, che sono in genere le clausole iniziali ad individuarne il campo di applicazione.

L'osservazione dei testi contrattuali dimostra come queste operazioni siano svolte con tecniche di redazione e con formule linguistiche diverse da settore a settore. Del resto, diverse sono le situazioni di fatto, tratte dal mondo dell'economia e del lavoro, che le parti sociali devono descrivere ed identificare, al fine di stabilire una disciplina collettiva. Di diversa intensità, inoltre, a seconda dei contesti, possono essere le esigenze di una (più o meno) puntuale definizione del campo di applicazione dei contratti collettivi, per evitare sovrapposizioni - o, al contrario, proprio per sovrapporsi surrettiziamente - con il campo di applicazione di altri accordi. Infine, non va trascurato che tali clausole dei contratti collettivi sono il frutto di un confronto tra le parti che non sempre perviene ad esiti realmente condivisi ${ }^{106}$ : con il risultato, in questi casi, di produrre disposizioni dalla portata ambigua, corredate di dichiarazioni a verbale o puntualizzazioni di vario genere, e di proseguire in definitiva il confronto sul terreno dell'interpretazione delle clausole contrattuali ${ }^{107}$.

Da questi rilievi di massima, può passarsi a qualche situazione più specifica. Ad esempio, continua a far discutere, in dottrina, la manipolazione dell'area contrattuale del Ccnl del credito del 1990, che ricomprese, al termine di una vertenza difficile ${ }^{108}$, le società controllate di diritto o di fatto dalle aziende associate alle organizzazioni stipulanti ${ }^{109}$. Anche da vicende meno controverse potrebbe-

106 G. GiugnI, La validità "erga omnes" dei contratti collettivi, in Nord e Sud, 1960, 2, pp. 74-75; Cfr. altresì O. MazzotTa, Divagazioni in tema di interpretazione del contratto collettivo, in R. Flammia (a cura di), L'interpretazione dei contratti collettivi di lavoro, Roma, Bancaria, 1999, p. 75.

107 Da tempo la dottrina più attenta alle dinamiche reali delle relazioni industriali ha rimarcato le difficoltà di attribuire interpretazioni lineari a «espressioni verbali non consuete né alla lingua dei puristi, né ai simboli concettuali o alle categorie proprie della dottrina, e neppure al più conciliante linguaggio legislativo» (così, G. GHEzzI, L'interpretazione delle norme delegate sul trattamento minimo dei prestatori di lavoro, in Riv. Trim. Dir. Proc. Civ., 1963, p. 351).

108 M. G. Garofalo, Decentramento produttivo, impresa-rete e area contrattuale dei bancari, in Riv. Giur. Lav., 1991, I, p. 71 ss.

109 La questione sollevò negli anni Novanta un vivacissimo dibattito dottrinale: AA.VV., Categorie professionali e contratti collettivi, cit., O. MAzzotTA, Leg- 
ro trarsi riscontri interessanti, su come l'autonomia collettiva organizza le proprie "tipizzazioni". Si andrebbe dalla lineare elencazione dei settori di attività per i quali è stipulato il Ccnl dell'industria alimentare $^{110}$, all'area contrattuale sostanzialmente aperta del Ccnl Multiservizi ${ }^{111}$, passando per il Ccnl dell'industria metalmeccanica, che definisce il campo di applicazione in un'esposizione articolata in ben sette pagine ${ }^{112}$ : a un'identificazione di massima dei settori si

ge e contratto collettivo nelle trasformazioni delle imprese, in AA.VV., Le trasformazioni aziendali in vista del Mercato europeo: legge e contratto collettivo, Roma, Bancaria, 1992, p. 52 ss., F. MAIOLINI, Rapporti di partecipazione societaria ed ambito di applicazione del contratto collettivo nel sistema creditizio, in Dir. Rel. Ind., 1993, p. 39 ss., P. Tuluini, La revisione del sistema di relazioni sindacali nelle banche, in Dir. Prat. Lav., 1998, pp. 1498-1500, F. LunARdon, Autonomia collettiva e gruppi di imprese, cit., p. 195 ss., P. LAMBERTUCCI, Area contrattuale e autonomia collettiva, cit., p. 305 ss. Dell'area contrattuale del settore del credito si continua, tuttavia, a discutere, ogni qual volta si affronti il tema dei rapporti tra trasformazioni dei processi economici e ambito di applicazione dei contratti collettivi: L. CoRAZZA, Contractual integration e rapporti di lavoro, Padova, Cedam, 2004, p. 240 ss., I. Alvino, Il lavoro nelle reti d'imprese: profili giuridici, Milano, Giuffrè, 2014, p. 284 ss., D. IzzI, Lavoro negli appalti e dumping salariale, Torino, Giappichelli, 2018, pp. 48-49.

${ }_{110}$ Si tratta attualmente del Ccnl 27 ottobre 2012 concluso tra Federalimentare, Aidepi, Aiipa, Ancit, Anicav, Assalzoo, Assica, Assitol, Assobibe, Assobirra, Assocarni, Assolatte, Federvini, Italmopa, Mineracqua, Unionzucchero, Fai-Cisl, Flai-Cgil e Uila-Uil.

111 Ccnl per il personale dipendente da imprese esercenti servizi di pulizia e servizi integrati/multiservizi del 31 maggio 2011 concluso tra Fise-Federazione imprese di servizi, Legacoop Servizi, Federlavoro e Servizi Confcooperative, Unionservizi Confapi, Agci Servizi e Filcams-Cgil, Fisascat-Cisl e Uiltrasporti-Uil. Invero, questo contratto collettivo è stato "ideato", nella stagione delle privatizzazioni, proprio per regolare un'ampia varietà di servizi, svolti in regime di esternalizzazione per conto di pp.aa. e di imprese private. L'art. 1 fornisce un'elencazione di tali servizi definita «esemplificativa», ma che l'area contrattuale sia sostanzialmente indeterminata, lo si desume più che altro dalla formula di chiusura, con la quale si prevede che sono «escluse dalla sfera di applicazione del contratto le eventuali autonome attività, anche per specifici contratti di committenza, ai rapporti di lavoro delle quali si applichino, secondo la vigente normativa, autonomi e specifici c.c.n.l. corrispondenti». Che tale area contrattuale sia difficilmente determinabile è considerazione che trova riscontro anche nella giurisprudenza: v., ad es., Cons. St., sez. V, $1^{\circ}$ marzo 2017, n. 932, massima in Giur. It., 2017, p. 1667, nota G. BARBERIS, testo integrale in Pluris.

112 Si tratta attualmente del Ccnl 26 novembre 2016 concluso tra Federmeccanica, Assistal, Fim-Cisl, Fiom-Cgil e Uilm-Uil. Cfr. A. Maresca, Il rinnov(ament) o del contratto collettivo dei meccanici: c'è ancora un futuro per il contratto collettivo nazionale di categoria, in Dir. Lav. Rel. Ind., 2017, p. 709 ss., V. Bavaro, Il 
aggiungono una descrizione «a titolo indicativo ed esemplificativo» di una serie di stabilimenti e di attività ricomprese nel contratto, un «chiarimento a verbale» (circa alcune attività «tradizionalmente» ricomprese nel metalmeccanico) e una «norma comune» (sui criteri per definire l'attività prevalente negli stabilimenti in cui se ne svolgano più d'una) ${ }^{113}$. Fino al caso sui generis del contratto collettivo dei giornalisti, che contempla, ai fini della propria applicazione, un requisito oggettivo (lo svolgimento dell'attività di giornalista e l'essere il datore di lavoro un editore) e un requisito soggettivo (la qualifica di giornalista iscritto all'albo) ${ }^{114}$.

In definitiva, si è colto nel segno col rilevare che ogni categoria possiede un proprio "stile" 115 o una propria "identità"116 nella definizione del campo di applicazione del contratto collettivo; non potrebbe essere diversamente: ciascuna di tali costruzioni della realtà socioeconomica è l'esito di un articolato gioco di relazioni tra organizzazioni collettive ${ }^{117}$, che perviene nel tempo ad esiti diversi ${ }^{118}$, «in base alla storia, alla ricchezza economica, alla forza contrattuale» ${ }^{119}$.

contratto nazionale dei metalmeccanici 2016: una prospettiva sulle relazioni industriali italiane, ivi, p. 729 ss.

113 Per un commento alle clausole sul campo di applicazione (rinnovo del 2008), cfr. A. LASSANDARI, Campo di applicazione del contratto, in M. G. GAROFALO, M. Roccella (a cura di), Commentario al contratto collettivo nazionale di lavoro dei metalmeccanici: 20 gennaio 2008, Bari, Cacucci, 2010, p. 29 ss.

114 G. Giugni, Lavoro giornalistico, in Enc. Dir., XXIII, 1973, p. 449: è una «delle caratteristiche più singolari della contrattazione collettiva dei giornalisti».

115 J.-F. Poisson, Rapport sur la négociation collective et les branches professionnelles, cit., p. 36.

116 A. Lassandari, Intervento, in Autonomia collettiva e occupazione. Atti del XII Congresso nazionale di diritto del lavoro Milano, 23-25 maggio 1997, Milano, Giuffrè, 1997, p. 204.

117 J. Saglio, La régulation de branche dans le système français de relations professionnelles, in Les relations sociales en Europe, Paris, Ministère du Travail et de l'Emploi, SES, 1990, p. 37.

118 R. MacKenzie, M. Martinez Lucio, The Realities of Regulatory Change: Beyond the Fetish of Deregulation, in Sociology, 2005, p. 505 ma passim.

119 Così, M. Pedrazzoli, Qualificazioni dell'autonomia collettiva e procedimento applicativo del giudice, cit., p. 554. 


\section{Principio di libertà sindacale e definizione delle unità negoziali}

La definizione di una sfera elettiva di inquadramento è oggetto di una tutela piena da parte del comma $1^{\circ}$ dell'art. 39 Cost., che deriva dalla stessa libertà di costituire organizzazioni sindacali (v. supra, § 3). D’altro canto, è un dato ormai acquisito alla riflessione giuridica che la tutela della norma costituzionale si riferisca non soltanto alle organizzazioni, ma anche alle loro attività, ivi compresa la contrattazione collettiva, anche se svolta in base al diritto comune dei contratti, in alternativa allo schema inattuato di cui all'art. 39, c. 4 , Cost. ${ }^{120}$.

In relazione al tema che ci occupa, si pone la questione di chiarire se ed in che modo quel particolare "momento" della contrattazione collettiva, con il quale le organizzazioni contrapposte definiscono le unità negoziali, sia protetto, in sé, dalla norma di garanzia della Costituzione. Occorre chiedersi, cioè, se nell'attività qualificatrice o, se si vuole, di tipizzazione dell'autonomia collettiva si possa ravvisare una situazione giuridica autonoma, rispetto al più generale svolgimento della contrattazione collettiva, che ne giusti-

120 Il ragionamento è in nuce in C. cost. 9 aprile 1969, n. 68, in Dir. Lav., 1970, II, p. 58, che dichiara l'illegittimità costituzionale delle disposizioni che sottraggono i lavoratori domestici alla disciplina della contrattazione collettiva (la Corte si arresta alla censura ex art. 3 Cost. e non entra in medias res sull'art. 39 Cost.), ma è apertamente svolto a partire dalle sentenze sui tetti massimi alla contrattazione collettiva (tra di esse, v. C. cost. 7 febbraio 1985, n. 34, in Giust. Civ., 1985, p. 607, nota G. Pera, Riv. It. Dir. Lav., 1985, II, p. 153, nota S. MagrinI) sul nulla-osta della Banca d'Italia per la contrattazione collettiva per le Casse di risparmio (C. cost. 24 marzo 1988, n. 330, in Riv. It. Dir. Lav., 1988, II, p. 568, nota V.A. Poso, oss. G. Pera) e, di recente, ripreso in materia di "blocco" della contrattazione collettiva nel settore pubblico (C. cost. 23 luglio 2015, n. 178, in Giur. Cost., 2015, p. 1651, nota G. Fiorillo, Dir. Rel. Ind., 2015, p. 1120, nota V. Ferrante, Dir. Lav. Merc., 2015, p. 377, nota L. ZopPOLI). Per l'orientamento dottrinale secondo il quale l'attività contrattuale di diritto comune delle associazioni sindacali di fatto è direttamente tutelata, nel diritto positivo, dal $1^{\circ}$ comma dell'art. 39 Cost., G. Pera, Problemi costituzionali del diritto sindacale italiano, cit., p. 106, R. Scognamiglio, Autonomia sindacale ed efficacia del contratto collettivo di lavoro, in Riv. Dir. Civ., 1971, p. 160, M. Persiani, Saggio sull'autonomia privata collettiva, cit., p. 38 ss., G. Giugni, Art. 39, in Giu. Branca (a cura di), Commentario alla Costituzione, Rapporti economici, I, Bologna-Roma, Zanichelli-Società editrice del Foro italiano, 1979, p. 257 ss. 
fichi una protezione, per così dire, diretta da parte del comma $1^{\circ}$ dell'art. 39 Cost.

Sia pure a costo di qualche schematismo, è possibile individuare due passaggi-chiave, nel consolidamento dell'opinione, avanzata apertamente per la prima volta da Esposito nel 1954 ${ }^{121}$, secondo la quale la definizione delle unità negoziali della contrattazione collettiva sia, in quanto tale, espressione di libertà sindacale.

\subsection{La giurisprudenza costituzionale sulla legge Vigorelli}

Il primo di tali passaggi può essere compreso con alcuni riferimenti all'elaborazione dottrinale e giurisprudenziale in materia di legge Vigorelli. La vicenda è fin troppo nota per dover essere ricapitolata $^{122}$. Il punto che interessa riguarda la nozione di categoria accolta dalla legge, la quale, al suo art. 1, delegava il Governo ad emanare norme di legge ai fini di «assicurare minimi inderogabili di trattamento economico e normativo nei confronti di tutti gli appartenenti ad una medesima categoria», uniformandosi «a tutte le clausole dei singoli accordi economici e contratti collettivi, anche intercategoriali, stipulati dalle associazioni sindacali anteriormente alla data di entrata in vigore della $[\ldots]$ legge».

La dottrina aveva da subito avvertito che si sarebbero prospettate alcune situazioni problematiche ${ }^{123}$. In primo luogo, nei casi di sovrapposizione tra gli ambiti di applicazione di contratti collettivi recepiti in decreto. Non si sarebbe trattato di casi frequenti (data la complessità tutto sommato circoscritta del sistema contrattuale dell'epoca, per lo meno rispetto a quello odierno), ma in quelle situazioni in cui si fosse in concreto posto il problema, si sarebbe potuta dare prevalenza a una soltanto tra le discipline di categoria in astratto applicabili? In caso di risposta affermativa, in base a quali criteri, dato che la legge non ne prevedeva alcuno? In secondo luo-

121 C. Esposito, Lo Stato e $i$ sindacati, cit.

122 Cfr. F. Santoro-Passarelli, La disciplina transitoria dei rapporti di lavoro, Roma, Edizioni Ricerche, 1961.

123 G. Giugni, La disciplina legislativa del trattamento minimo di categoria, in Riv. Trim. Dir. Proc. Civ., 1959, p. 863 ss. 
go, si poneva il problema della vincolatività dei contratti collettivi recepiti in decreto per le aziende artigiane. In particolare, per il caso in cui, pur svolgendo attività ricomprese negli ambiti di applicazione dei contratti collettivi di categoria stipulati per le aziende industriali, esse fossero inquadrate in associazioni estranee alla stipulazione dei contratti collettivi; oppure, nel caso in cui, pur non essendo sindacalizzate, esse si ritenessero comunque estranee alle aree contrattuali stabilite dai contratti collettivi di area industriale ${ }^{124}$.

In sintesi, si poneva il problema se la disciplina delegata avrebbe potuto (senza prevederlo espressamente) alterare le determinazioni sugli ambiti di applicazione dei contratti collettivi recepiti in decreto, allo scopo di renderle, per così dire, più aderenti alle regolande realtà economico-produttive.

In relazione alla prima ipotesi, di coesistenza di più contratti collettivi con ambiti di applicazione sovrapposti ${ }^{125}$, la Corte costituzionale risolse la questione affermando l'illegittimità dei relativi d.p.r. di recepimento ${ }^{126}$. In assenza di criteri di preferenza nella legge delega, il legislatore delegato si sarebbe dovuto semplicemente astenere dall'operare tra di essi una scelta o, come era avvenuto, dal recepire entrambi i contratti collettivi. Come dire, in altri termini, che il funzionamento del meccanismo previsto dalla legge $n$. 741/1959 presupponeva il corretto svolgimento dell'ordinamento intersindacale, ossia, tra le altre cose, l'esistenza di una ripartizione ordinata delle unità negoziali della contrattazione collettiva.

La seconda situazione era più complessa. Al riguardo, erano emerse due principali posizioni in dottrina. Un primo orientamento

124 Questa, invero, è una rappresentazione semplificata di questioni assai più articolate di politica retributiva e di appartenenza sindacale, che interessavano già allora le aziende artigiane e, più in generale, il vasto mondo delle piccole imprese, in relazione all'area di rappresentanza confindustriale e che avrebbero in futuro continuato a riproporsi (cfr. A. Perulli, L'attività sindacale nella piccola impresa, in AA.VV., Piccola impresa e diritto del lavoro, Quaderni di diritto del lavoro e delle relazioni industriali, Torino, Utet, 1991, p. 163 ss.).

125 Nella specie, si trattava dei contratti collettivi conclusi per i dipendenti delle aziende lattiero casearie da una parte (categoria per così dire più "ampia", per le tipologie di attività in essa ricomprese) e per i dipendenti delle centrali del latte e dei centri di trattamento e di confezionamento del latte alimentare (categoria se si vuole più "ristretta", ossia volta a regolare un numero inferiore di attività).

126 C. cost. n. 106/1963, cit. 
sosteneva che l'art. 1 avesse accolto una nozione "merceologica" di categoria (semplificando: settore metalmeccanico, edilizia, ecc.). Da qui, si deduceva che tutti i datori di lavoro operanti nel ramo dell'economia (che svolgevano, cioè, quel tipo di attività), per il quale era stato stipulato un contratto collettivo recepito in decreto sarebbero stati vincolati dalla disciplina estesa erga omnes, quale che ne fosse stato in origine il campo di applicazione ${ }^{127}$. Il discrimine, cioè, sarebbe stato costituito dai "confini" del settore economico e non da quelli delle unità negoziali dei contratti collettivi. Per un secondo orientamento, $i$ contratti collettivi recepiti in decreto sarebbero stati resi obbligatori esclusivamente per i soggetti ricompresi nei loro ambiti di applicazione, in base a un'interpretazione rimessa al giudice delle clausole sulla determinazione delle aree contrattuali ${ }^{128}$. Quali che fossero i confini dei settori economici è alle clausole (sul campo di applicazione) dei contratti collettivi recepiti in decreto che si sarebbe dovuto guardare, per definire l'ambito di obbligatorietà delle discipline estese erga omnes. La legge non avrebbe potuto mutare, in modo surrettizio, le unità negoziali definite dai contratti collettivi in categorie merceologiche o produttive, senza violare il principio di libertà sindacale $\mathrm{o}$, comunque, senza condurre a conseguenze incongrue ${ }^{129}$.

A prevalere fu la seconda tesi: nelle parole di Giugni, «estendere erga omnes vuol dire, semplicemente, applicare la disciplina contenuta nel contratto anche ai non iscritti, ma pur sempre nella cornice di efficacia che il contratto si è dato secondo la volontà dei

127 Questo orientamento era difeso specialmente dalla Riv. Giur. Lav.: cfr., per tutti, U. Natoli, Alcuni rilievi in vista dell'attuazione della legge 15 luglio 1959, n. 741, in Riv. Giur. Lav., 1959, I, p. 103 ss.

128 Propendevano per questo (prevalente) orientamento: G. GIUGNI, La disciplina legislativa del trattamento minimo di categoria, cit., pp. 879-884, G. VINCENZI, R. Flammia, Le norme transitorie per garantire minimi di trattamento economico e normativo ai lavoratori, in Mass. Giur. Lav., 1959, pp. 122-123, V. Carullo, Diritto sindacale transitorio, Milano, Giuffrè, 1960, pp. 68, 203 s., G. Mazzoni, I limiti di applicabilità dell'art. $2070 \mathrm{cod}$. civ. ed il potere sindacale di valutazione degli interessi collettivi, in Mass. Giur. Lav., 1964, p. 107 ss.

129 V., in tal senso, A. CESSARI, L'interpretazione dei contratti collettivi, Milano, Giuffrè, 1963, pp. 94-95, 116 ss. 
contraenti ${ }^{130}$. La Corte costituzionale, pur senza risolvere apertamente la questione controversa della vincolatività dei contratti collettivi rispetto alle aziende artigiane, affermò che sarebbe spettato al giudice ordinario accertare i confini della categoria contrattuale rispetto alle tipologie di attività in esse ricomprese, mediante un'interpretazione delle clausole negoziali. È a questo proposito che la Corte precisò che il legislatore non ha sostituito le categorie che «si sono spontaneamente definite mediante l'autonomo ordinamento sindacale e mediante la stipulazione di contratti collettivi», con altre «sulla base di un'astratta identificazione di attività merceologiche o sulla scorta di altri criteri arbitrariamente prestabiliti ${ }^{131}$.

Invero, se a distanza di alcuni anni si poté affermare che dall'esperienza applicativa della legge «l'autonomia contrattuale dei sindacati non era uscita né distrutta né mortificata ${ }^{132}$, lo si deve anche al fatto che tale autonomia fu preservata in uno dei suoi profili sostanziali: la determinazione delle aree contrattuali. Anzi, proprio la controversa esperienza della legge Vigorelli aveva condotto la giurisprudenza costituzionale ad affermare apertamente che la determinazione del campo di applicazione dei contratti collettivi fosse essa stessa protetta dal principio di libertà sindacale. Atteso che la definizione delle unità negoziali «si assume e si ammette conforme alla dinamica delle forze del lavoro ed alla struttura delle imprese», una loro alterazione «porterebbe alla violazione del principio di libertà e di autonomia sindacale» ${ }^{133}$.

130 Così, G. Giugni, Osservazioni sulle sentenze n. 70 e 106 della Corte costituzionale, cit., p. 827.

131 C. cost. n. 70/1963, cit. Come si potrà intuire, il problema della vincolatività per le aziende artigiane dei contratti collettivi per ramo d'industria estesi erga omnes non si poté considerare risolto, atteso che non sempre l'interpretazione del testo contrattuale avrebbe fornito risposte univoche circa la ricomprensione o meno di tali imprese nelle aree contrattuali. Non stupisce che nella giurisprudenza successiva si registrino pronunce di segno contrastante: v. ad esempio Cass. III Sez. pen. 23 aprile 1965, n. 1318, in Riv. Dir. Lav., 1966, II, p. 9 ss., nota P. GuaLTIEROTTI.

132 Così, U. Romagnoli, Il contratto collettivo difficile (1971), in ID., Lavoratori e sindacati tra vecchio e nuovo diritto, Bologna, Il Mulino, 1974, pp. 215-216.

133 C. cost. n. 106/1963, cit. 


\subsection{L'affermazione di una nozione volontaristica di categoria}

Le vicende della legge Vigorelli, come noto, culminarono nella dichiarazione di incostituzionalità della legge di proroga (n. 1027) approvata dal Parlamento il $1^{\circ}$ ottobre 1960, per reiterare il meccanismo di recepimento dei contratti collettivi sperimentato nel $1959^{134}$.

Ora, si potrebbe anche supporre che se il legislatore avesse, dopo tali vicende, emanato una disciplina attuativa dell'art. 39, c. 2 ss., Cost., sarebbe stata ridimensionata la portata della giurisprudenza costituzionale in materia di definizione delle unità contrattuali, che era pur sempre scaturita da questioni applicative della legge Vigorelli: ciò, si badi, non nel principio di fondo - di una sfera di autonomia delle parti sociali in questa materia -, che è inscindibile dalla garanzia di libertà sindacale, bensì in alcuni risvolti pratici, come i meccanismi mediante i quali pervenire a una ripartizione ordinata del sistema di contrattazione collettiva in unità negoziali ${ }^{135}$. La previsione di tali meccanismi non può che implicare l'esistenza di un bilanciamento ${ }^{136}$ tra ragioni di certezza giuridica e libertà delle parti sociali di determinare in autonomia le aree contrattuali ${ }^{137}$ : «la libertà inizialmente affermata - scriveva Pera nella sua celebre monografia del 1960 - è suscettiva di ridursi concretamente di quel tanto o di quel poco che il complessivo congegno esige» ${ }^{138}$.

Tuttavia, il fatto stesso che ciò non sia accaduto ha innegabilmente rilevanza: il valore in senso lato normativo della mancata attuazione dell'art. 39 Cost. è uno dei motivi ricorrenti dell'intera elaborazione gius-sindacale italiana. Da tale inattuazione - si è soliti

134 C. cost. n. 106/1962, cit.

135 È la stessa Corte costituzionale, nella sentenza n. 106/1963, cit., a prendere in considerazione, in un passaggio della motivazione, la possibilità che il quadro possa cambiare, in questa materia («quando ancora non è stata data esecuzione al precetto del quarto comma dell'art. 39 Cost.»). V. in dottrina F. Liso, Categorie e qualifiche del lavoratore, cit., p. 3.

136 Sul bilanciamento tra principi costituzionali, cfr. L. NogLER, La disciplina dei licenziamenti individuali nell'epoca del bilanciamento tra $i$ "principi" costituzionali, in Dir. Lav. Rel. Ind., 2007, p. 593 ss.

137 G.F. MANCINI, Libertà sindacale e contratto collettivo "erga omnes", cit., p. $140 \mathrm{ss}$.

138 G. Pera, Problemi costituzionali del diritto sindacale italiano, cit., p. 59. 
affermare - non è derivato soltanto un vuoto di disciplina, ma si sono anche dipartiti, ad opera di dottrina e giurisprudenza, una serie di percorsi ricostruttivi che il costituente non aveva preconizzato $^{139}$. Questa considerazione conduce, in sostanza, ad affermare che il consolidamento del sistema negoziale di fatto, su linee che proprio in quegli anni iniziavano a percepirsi con lo sviluppo della contrattazione articolata, ha contribuito in modo decisivo alla maturazione del convincimento che la configurazione delle unità negoziali rientri toto coelo tra le prerogative "sovrane" dell'autonomia collettiva ${ }^{140}$.

Non è un caso, insomma, che il secondo passaggio-chiave per il consolidamento di questa opinione sia coinciso con il processo di articolazione su più livelli della struttura contrattuale, avviatosi nella seconda metà degli anni Cinquanta del secolo scorso, id est con la "apertura" di nuove unità negoziali, ricavate dalla dimensione dell'impresa o dell'unità produttiva ${ }^{141}$.

A quel tempo, lo sviluppo tecnologico dell'industria, col rendere obsolete le classificazioni delle mansioni contenute nei contratti di settore (spesso riprese in modo tralatizio dai precedenti corporativi) apriva rapidamente la strada ad una negoziazione svolta per il perimetro aziendale ${ }^{142}$. Nel dibattito giuridico si poneva, tuttavia, la

139 Per tali ragionamenti, si vedano specialmente i contributi di G.F. ManCINI, Sindacato e Costituzione trent'anni dopo, in ID., Costituzione e movimento operaio, cit., p. 163 ss., S. Sciarra, l'orgoglio dell'inattuazione costituzionale, in Pol. Dir., 1985, p. 421 ss., G. PERA, Il trentanovismo è nelle cose, ivi, p. 503 ss., M. D’AnTonA, Il quarto comma dell'art. 39 della Costituzione, oggi, in Dir. Lav. Rel. Ind., 1998, p. 665 ss.

140 Simili andamenti emergono con chiarezza dalle parole di R. DE LucA TAMAJO, L'evoluzione dei contenuti e delle tipologie della contrattazione collettiva, in Riv. It. Dir. Lav., 1985, I, p. 18: «la mancata adozione di una legge sindacale ha fatto sì che sotto il profilo dei contenuti dei contratti collettivi, l'autonomia sindacale potesse muoversi in completa autonomia»; «l'art. 39, comma $1^{\circ}$, Cost., nel sancire il principio di libertà sindacale, garantisce, implicitamente ma inscindibilmente, la libertà dei contenuti, degli svolgimenti e dei livelli di svolgimento dell'autonomia collettiva che, nel testo costituzionale come nella prassi delle relazioni industriali, assume la configurazione di un potere sociale non predeterminato nei fini e negli oggetti e perciò caratterizzato da una possibilità di esplicazione su una pluralità di versanti e nei confronti di una molteplicità di antagonisti».

141 Mette in luce la relazione tra sviluppo della contrattazione articolata e rinnovamento del concetto tradizionale di categoria nel diritto sindacale, G. TARELLO, Teorie e ideologie del diritto sindacale, cit., p. 101 ss.

142 F. Momigliano, Sindacati, progresso tecnico, programmazione economica, Torino, Einaudi, 1966, p. 127 ss. 
questione della configurabilità di una contrattazione collettiva che si svolgesse su un piano altro da quello delle unità aggregate per setto$\mathrm{re}^{143}$. Si poneva, cioè, il problema se dall'ordinamento giuridico (in specie, art. 39 Cost.) potesse ricavarsi una nozione preconcetta di categoria contrattuale, che si sarebbe inverata nella contrattazione collettiva di settore o per branca produttiva. Il che avrebbe impedito agli attori sociali di negoziare veri e propri contratti collettivi in ambiti diversi ${ }^{144}$. L'esito della vicenda in sé è noto: prevalse la posizione che non potesse ricavarsi dall'ordinamento giuridico una nozione ontologica di categoria contrattuale; di categoria non si sarebbe potuto parlare se non come risultato di una negoziazione collettiva svolta per unità nelle quali le parti contrapposte si fossero riconosciute come validi interlocutori ${ }^{145}$.

L'organizzazione sindacale e la contrattazione collettiva sono il prius, le categorie il posterius, per riprendere un passaggio, rimasto celebre, della prolusione bolognese di Mancini del 1962, con il quale si affermava la libertà del soggetto storico di modellare arbitrariamente la propria presenza nella realtà industriale e di giocarvi un ruolo non predeterminato negli ambiti e nei livelli di intervento. $\grave{E}$ un rovesciamento del procedimento metodologico del diritto sindacale e una rivoluzione «dei suoi principi o di quello che prima era ritenuto tale» ${ }^{146}$, che investe in pieno la nozione tradizionale di categoria, determinandone la crisi.

In questa sistemazione, la definizione delle unità negoziali della contrattazione collettiva diviene «parte essenziale della libertà di associazione sindacale» ${ }^{147}$ o, se si vuole, corollario ${ }^{148}$ dell'individuazione, da parte delle organizzazioni sindacali, di una sfera elettiva di

143 V. la ricostruzione di S. Liebman, Autonomia collettiva e legge, in M. D’Antona (a cura di), Letture di diritto sindacale, Napoli, Jovene, 1990, pp. 51-71.

144 Su questi passaggi, cfr. R. Del PunTA, Il contratto aziendale nella dottrina italiana, in Riv. It. Dir. Lav., 1989, I, p. 258 ss.

${ }_{145}$ Tra i molti, Gio. Branca, La prestazione di lavoro in società collegate, Milano, Giuffrè, 1965, p. 94.

146 Così, G. TARello Teorie e ideologie nel diritto sindacale, cit., p. 103.

147 Si trae da C. cost. n. 88/1965, cit.

148 T. TREU, Regole e procedure nelle relazioni industriali: retaggi storici e criticità da affrontare, cit., p. 11. 
intervento nel mondo dell'economia e del lavoro' ${ }^{149}$. Entrambe le situazioni, in tal senso, sono percepite come momenti distinti ma inestricabilmente connessi di una medesima esperienza sindacale, che può dispiegarsi liberamente nella realtà socioeconomica con la protezione «diretta» ${ }^{150}$ del primo comma dell'art. 39 Cost. ${ }^{151}$.

Si giunge, così, a un'impostazione che, nei suoi postulati essenziali, non sarà più rimessa in discussione: la categoria contrattuale è il prodotto di una libera valutazione delle parti contrapposte, le quali, per un'esigenza logica prima che giuridica, per darsi una disciplina collettiva delle condizioni di lavoro, devono poterne individuare i potenziali destinatari. È opinione comune che la definizione delle aree contrattuali sia tra $\mathrm{i}$ «più delicati e rilevanti momenti di espressione» ${ }^{152}$ dell'autonomia collettiva $\mathrm{o}$, per altri, «una delle prime - e più gelosamente difese - prerogative desumibili dal principio di [...] libertà sindacale» ${ }^{153}$. Tale attività è la proiezione, sul piano negoziale, della rappresentatività che le parti dei contratti collettivi reciprocamente si riconoscono, per una sfera di interessi produttivi e di lavoro. Essa è altresì lo strumento con il quale la contrattazione collettiva adatta le proprie configurazioni strategiche alle strutture di mercato e dei processi produttivi e ai mutamenti nella composizione della forza lavoro $^{154}$. La situazione giuridica che consiste nella definizione delle unità

149 La stessa diffusione, all'epoca, della terminologia in uso nel Regno Unito e negli Stati Uniti (v. supra, § 4) è indicativa di come tra i giuristi delle «nuove scuole» (G. Pera, Libertà sindacale (diritto vigente), in Enc. Dir., XXIV, 1974, p. 519) si alludesse, fin dal linguaggio, e in particolar modo con l'uso del vocabolo "giurisdizione", all'esistenza di una sfera di autonomia dei sindacati che si estende tanto sul piano della struttura organizzativa quanto su quello della contrattazione collettiva.

150 E. GHERA, Le c.d. clausole sociali: evoluzione di un modello di politica legislativa, in Dir. Rel. Ind., 2001, p. 151. pp. 67-68.

151 L. Riva Sanseverino, Contratto collettivo di lavoro, in Enc. Dir., X, 1962,

152 Così, M. Barbieri, (M.G. Garofalo), La contrattazione collettiva, in F. CARINCI (dir. da), Il lavoro alle dipendenze delle amministrazioni pubbliche dal D.LGS. 29/1993 alla Finanziaria 1995. Commentario, I, Milano, Giuffrè, 1995, p. 697.

153 Così, A. Lassandari, Pluralità di contratti collettivi nazionali per la medesima categoria, in Lav. Dir., 1997, p. 291.

154 T. Treu, La CISL degli anni 50 e le ideologie giuridiche dominanti, in G. TAREllo (a cura di), Dottrine giuridiche e ideologie sindacali, Materiali per una storia della cultura giuridica, III/2, Bologna, Il Mulino, 1973, pp. 303-304. 
negoziali della contrattazione collettiva è dotata, in breve, di un'autonomia concettuale che la rende, in quanto tale, meritevole di tutela da parte dell'ordinamento giuridico. Tale situazione è espressione di libertà, come lo è tout court la contrattazione collettiva, e si è invero colto nel segno avvertendo che è in quel momento che precisamente si celebra il principio della libertà sindacale ${ }^{155}$.

7. La sovrapposizione tra gli ambiti di applicazione dei contratti collettivi di categoria: un fenomeno relativamente nuovo

Le clausole sul campo di applicazione dei contratti collettivi attribuiscono alle ipotesi di fatti da esse previsti una "rilevanza sociale”, per il sistema di relazioni industriali: riconoscendosi come interlocutori (rappresentativi), i soggetti che stipulano i contratti collettivi manifestano, per così dire, apertamente, agli altri attori negoziali, la propria giurisdizione o, se si preferisce, la propria competenza, per un determinato segmento del mondo dell'economia e del lavoro.

Osservate nel loro complesso, le tipizzazioni dell'autonomia collettiva dovrebbero idealmente dare luogo a una serie di "insiemi", in grado di ricomprendere al loro interno una parte più o meno consistente del tessuto produttivo. Nell'esperienza italiana, si è soliti osservare come la rete costituita dalla contrattazione collettiva includa la gran parte, se non la quasi totalità, dei processi economici e produttivi svolti nel Paese ${ }^{156}$.

155 M. Pedrazzoli, Qualificazioni dell'autonomia collettiva e procedimento applicativo del giudice, cit., p. 553. Riepilogando, sul tema de quo è ravvisabile una sostanziale convergenza tra le posizioni espresse dalla dottrina e dalla giurisprudenza in età repubblicana. V., in aggiunta alla ricostruzione operata nel paragrafo precedente, soffermatasi in particolare sulla giurisprudenza costituzionale, Cass. 16 gennaio 1986, n. 260, in Dir. Lav., 1986, II, p. 233, per la quale «Rientra nell'autonomia collettiva, costituzionalmente garantita (art. 39, $1^{\circ}$ comma, cost., la definizione della categoria contrattuale che delimita l'ambito soggettivo di efficacia potenziale della parte normativa del contratto collettivo ed individua quindi i soggetti dei rapporti di lavoro nei cui confronti il contratto suddetto possa svolgere la sua funzione di predeterminazione del contenuto di contratti individuali».

156 È possibile di ciò fornire una dimostrazione indiretta, ma attendibile, con qualche riferimento al dibattito scaturito dalla disposizione della 1. n. 183/2014 (art. 1, c. 7, lett. g) che delegava il Governo ad introdurre un compenso orario mi- 
In certa misura, è inevitabile che vi siano intersezioni tra le unità negoziali della contrattazione collettiva. Benché esse corrispondano in linea di massima a settori distinti di attività economica, è invero la stessa demarcazione tra i settori a non essere sempre chiarissima ${ }^{157}$, specialmente nei periodi caratterizzati da rapida evoluzione tecnologica e dei processi produttivi ${ }^{158}$. Anche a voler trascurare gli effetti del pluralismo organizzativo, gli attori sociali non sempre potranno aggiustare con tempismo le proprie strutture all'evoluzione dell'economia. In breve, le linee di sviluppo del sistema di contrattazione collettiva e dell'economia sono solo tendenzialmente sovrapponibi$\mathrm{li}^{159}$ e sarebbe irrealistico pensare di poter tracciare con precisione geometrica i confini tra le unità negoziali.

Tuttavia, come regola di fondo, la struttura contrattuale dovrebbe essere configurata dalle parti sociali in modo da evitare che in più d'una unità negoziale ci si occupi della medesima tipologia di attività economica. Detto altrimenti, una situazione di fatto non dovrebbe, a rigore, essere oggetto di più d'una disciplina collettiva, se non in casi marginali, e possibilmente in presenza di criteri di governo

nimo per i lavoratori subordinati, nei «settori non regolati da contratti collettivi sottoscritti dalle organizzazioni sindacali dei lavoratori e dei datori di lavoro comparativamente più rappresentative sul piano nazionale»: al proposito, basterà ricordare che la dottrina da subito osservò che i contratti collettivi stipulati dalle organizzazioni storiche «regolano $\mathrm{i}$ rapporti di lavoro nella quasi totalità delle attività produttive del nostro paese» (così, V. Speziale, Le politiche del lavoro del Governo Renzi: il Jobs Act e la riforma dei contratti e di altre discipline del rapporto di lavoro, in WP CSDLE "Massimo D'Antona".IT, 233/2014, p. 48), dato che la contrattazione collettiva è «presente, in quasi tutte, se non in tutte, le categorie» (così, T. Treu, $I l$ salario minimo: limiti della supplenza giurisprudenziale e prospettive, in Giur. It., 2015, p. 747). Nello stesso senso, L. Bellard, Sistema politico, legge e relazioni industriali: dalla promozione all'esclusione?, in AA.VV., Studi in memoria di Mario Giovanni Garofalo, 1, cit., pp. 110-112.

157 Il rilievo è risalente nella dottrina economica (M. WoLfe, The concept of economic sector, in Quart. J. Eco., 1955, 3, p. 402 ss.) così come in quella giuridica (A. Orsi Battaglini, Relazioni sindacali e fonti normative nella legge quadro sul pubblico impiego, in A. Orsi Battaglini et al., Accordi sindacali e legge quadro sul pubblico impiego, Milano, Giuffrè, 1984, p. 11).

158 Anche questo è un rilievo risalente nella dottrina: $\mathrm{v}$. a questo proposito gli spunti di riflessione di G. Ferraro, La classificazione delle imprese ai sensi dell'art. 49 della legge n. 88/1989, in Riv. It. Dir. Lav., 1990, I, pp. 162-163.

159 B. Bechter, B. Brandl, G. Meardi, Sectors or countries? Typologies and levels of analysis in comparative industrial relations, in Eur. J. Ind. Rel., 2012, 3, p. 192. 
di conflitti che - ricorda Caruso ${ }^{160}$ - sono conflitti di rappresentanza prima che tra contratti collettivi (applicabili).

La regola di tendenziale unicità del contratto collettivo nel proprio campo di applicazione permette, in pratica, che non vi siano eccessive ambiguità sui livelli di trattamento economico e normativo dei rapporti di lavoro che il mercato considera adeguati, settore per settore, per voce degli attori delle relazioni industriali. Di ciò è a ben vedere un esempio la stessa "funzione" del Ccnl, per come individuata dai più importanti accordi interconfederali di questi ultimi anni: «garantire la certezza dei trattamenti economici e normativi comuni per tutti i lavoratori del settore ovunque impiegati sul territorio nazionale» (si trae dal Testo unico sulla rappresentanza 10 gennaio 2014 Confindustria e Cgil, Cisl e Uil, parte III, ma si ritrovano formule analoghe in altri accordi interconfederali). In questa formula, la parola settore sembra essere utilizzata in modo volutamente ambiguo, per rappresentare sia l'unità negoziale sia il settore economico e rimarcare, così, il valore delle discipline collettive come standard minimo di trattamento per tutti coloro che svolgono una certa attività ${ }^{161}$.

Peraltro, con la maggiore complessità che hanno acquisito, nel tempo, i sistemi di relazioni industriali, è diventato evidente come l'esigenza di una ripartizione ordinata delle aree di influenza organizzativa e negoziale delle associazioni sindacali e dei datori di lavoro non sia confinata all'espletamento della sola funzione cd. normativa dei contratti collettivi: si pensi, ad esempio, ai problemi derivanti dalla sovrapposizione tra gli ambiti di operatività degli enti bilaterali ${ }^{162}$. Più in generale, del resto, una ripartizione del sistema contrattuale su unità negoziali ben delimitate consente, alle organizzazioni dei lavoratori non meno che a quelle dei datori di lavoro, di evitare un'eccessiva competizione interna, tra strutture appartenenti

160 B. CARuso, Per un intervento eteronomo sulla rappresentanza sindacale: se non ora quando!, cit., pp. 6-7.

161 Il che equivale a dire che le parti sociali danno per presupposta la «identificazione della categoria con la platea dei lavoratori dipendenti da aziende a loro volta ricomprese in un medesimo settore merceologico o comparto produttivo»: A. Viscomi, Soggettività sindacale e categorie contrattuali, cit., p. 79.

162 L. Bellard, Il sistema di rappresentanza imprenditoriale e la struttura della contrattazione collettiva, in M. CARRIERI, T. TREU (a cura di), Verso nuove relazioni industriali, Bologna, Il Mulino, 2013, p. 259. 
ad una stessa confederazione, oltre che tra diverse confederazioni ${ }^{163}$. Infatti, la possibilità di estendere il raggio di azione negoziale, verso ambiti ricompresi in altri contratti collettivi, significa anche ampliare, oltre alla presa sul sistema economico-produttivo, il bacino di potenziali iscritti o di fruitori dei servizi di assistenza ${ }^{164}$ : una questione invero non da poco, in un'epoca in cui i tassi di sindacalizzazione, sia tra la forza lavoro sia tra i datori di lavoro, destano costante preoccupazione ${ }^{165}$.

La ripartizione del sistema contrattuale su unità negoziali non sovrapposte è stata a lungo uno dei presupposti di fatto dell'esperienza italiana di relazioni industriali. La comprensibile esistenza di eccezioni ${ }^{166}$ finiva in qualche modo per confermare la regola di fondo della tendenziale "unicità" dei contratti collettivi nel proprio campo di applicazione. La tipica frammentazione organizzativa del sistema sindacale, sia dal lato dei lavoratori sia da quello dei datori di lavoro, non aveva, cioè, impedito di pervenire ad una distribuzione della contrattazione collettiva su un numero ampio, aperto - per definizione - ma in fondo circoscritto di unità negoziali. Nel 1990, quando si iniziavano appena ad avvertire le trasformazioni con le quali oggi fa i conti il sistema di relazioni industriali ${ }^{167}$, era ancora possibile osservare che «nel nostro ordinamento sindacale di fatto, per i diversi ambiti di riferimento e in particolare per le categorie, vige un unico contratto collettivo, nonostante il pluralismo» ${ }^{168}$. Altri aggiungeva: l'idea «suggestiva della suddivisione del mondo del

163 M. ForLIVESI, La rappresentatività datoriale: funzioni, modelli, indici di accertamento, in Lav. Dir., 2018, p. 521 ss.

164 F. SCARPELl, Linee e problemi dei fenomeni di esternalizzazione e decentramento produttivo, in M. Brollo (a cura di), Il mercato del lavoro, in F. CARINCI, M. Persiani (dir. da), Trattato di diritto del lavoro, Padova, Cedam, 2012, p. 1448.

165 M. Carrieri, P. Feltrin, Al bivio. Lavoro, sindacato e rappresentanza nell'Italia d'oggi, Roma, Donzelli, 2016.

166 Si pensi ai dubbi, di cui la dottrina si occupava già nei primi anni Sessanta del secolo scorso, generati dalla coesistenza, per una medesima tipologia di attività economica, o per attività economiche non sempre nettamente distinguibili, di contratti collettivi per l'industria, per la piccola e media impresa e per l'impresa artigiana (cfr. B. BALLETTI, Contributo alla teoria della autonomia sindacale, Milano, Giuffrè, 1963, pp. 247-248).

167 AA.VV., Categorie professionali e contratti collettivi, cit.

168 Così, M. Pedrazzoli, Qualificazioni dell'autonomia collettiva e procedimento applicativo del giudice, cit., p. 395. 
lavoro e della economia per categorie e la buona tenuta del sistema sindacale su di esse imperniato bastano a contenere entro termini ragionevoli le inevitabili disfunzioni di un sistema aperto» ${ }^{169}$.

Tuttavia, ciò non corrisponde più alla situazione esistente nel sistema di contrattazione collettiva (del settore privato). Lo dimostrano il numero spropositato di contratti collettivi depositati presso l'archivio pubblico dei contratti del Cnel e, indirettamente, gli stessi tentativi delle parti sociali di riportare ordine nel sistema contrattuale, mediante la conclusione di accordi interconfederali su temi nevralgici come la misurazione della rappresentatività e i criteri di maggioranza per la stipulazione dei contratti collettivi. L'accentuarsi del pluralismo, anche - se non soprattutto - tra i datori di lavo$\mathrm{ro}^{170}$, si riflette oggi in una sovrapposizione tra gli ambiti di applicazione dei contratti collettivi che supera un livello da considerare fisiologico, se non ineliminabile, in ogni sistema di relazioni industriali. Specialmente nei comparti dell'economia in cui più esasperata è la segmentazione delle attività d'impresa e della forza lavoro ${ }^{171}$, la regola di unicità dei contratti collettivi nel proprio campo di applicazione è divenuta instabile, se non appena tendenziale, con ricadute sul piano del conflitto collettivo ${ }^{172}$, anche nei servizi essenziali ${ }^{173}$.

$\grave{E}$ in questi contesti che le trasformazioni dei processi econo-

169 Così, R. Scognamiglio, Intervento, in AA.VV., Categorie professionali e contratti collettivi, cit., p. 131.

170 P. Albini, A proposito di rappresentanza datoriale, in Dir. Lav. Rel. Ind., 2017, p. 265 ss.

171 T. TREU, Il conflitto e le regole, in Dir. Lav. Rel. Ind., 2000, pp. 314, 322; v. altresì M. MAGNANI, La disciplina dello sciopero nei servizi pubblici essenziali alla prova dei fatti, in Riv. It. Dir. Lav., 2005, I, pp. 69-70.

172 Per il caso emblematico dell'industria alimentare v. U. Franciosi, Il caso Castelfrigo, in A. Allamprese, O. Bonardi (a cura di), Logistica e lavoro, cit., pp. 131-132.

173 V. ad esempio la Relazione annuale 2019 sull'attività svolta nell'anno 2018 della Commissione di garanzia (p. 31), nella quale, in relazione al settore dei servizi di igiene ambientale, tra le cause di insorgenza dei conflitti, si identifica (per l'Italia settentrionale) la «sempre più pronunciata frammentazione e segmentazione del servizio, con il massiccio ricorso al subappalto ed il fiorire di Cooperative sociali che operano sul territorio con il ricorso a mano d'opera a basso costo oppure applicando alle maestranze storiche, protette dalle clausole sociali, contratti peggiorativi; nel settore è, ormai, comune la pratica di applicare agli operatori ecologici il C.C.N.L. Pulizie e Multiservizi, totalmente estraneo alle mansioni effettivamente svolte dai lavoratori». 
mici si sono risolte in un «gioco di specchi», nel quale è «difficilissimo esercitare l'attività sindacale» e che in definitiva «mette in crisi alcune nozioni tipiche delle relazioni industriali, come il concetto di categoria merceologica, su cui si è sviluppata storicamente la nostra contrattazione collettiva di livello nazionale» ${ }^{174}$.

\section{Inquadramento delle questioni da affrontare e struttura del lavoro}

Dato questo punto di partenza, la ricerca si articolerà di qui in avanti su tre capitoli ed altrettanti piani di ragionamento.

Il prossimo capitolo (II) esaminerà il problema della categoria in una "prospettiva interna alle relazioni industriali". Data la consolidata tradizione volontaristica del sistema italiano, e la diretta rilevanza del principio di libertà sindacale nelle attività di definizione delle unità negoziali della contrattazione collettiva, non può che essere questo il primo passaggio della riflessione. In particolare, si cercherà di fare il punto sullo "stato dell'arte" del sistema di contrattazione collettiva del settore privato, con riferimento alla sovrapposizione tra gli ambiti di applicazione dei contratti collettivi. Si porrà l'attenzione, da un lato, sulla proliferazione di accordi collettivi "pirata”, stipulati tra coalizioni negoziali dalla dubbia rappresentatività rispetto al tessuto produttivo delle imprese ed alla forza lavoro; dall'altro, sui casi (più frequenti di quanto talvolta non si pensi) di intersezione tra gli ambiti di applicazione dei contratti collettivi stipulati all'interno dello stesso circuito della rappresentanza confederale. In seguito, ci si soffermerà sui tentativi che, tra molte difficoltà, gli attori più accreditati del sistema di relazioni industriali stanno portando avanti in questi anni, allo scopo di rinnovare il compromesso che si pone alla base della contrattazione collettiva e rivedere i confini delle sfere di influenza organizzativa e negoziale delle confederazioni e delle federazioni di categoria. L'obiettivo di una semplificazione del sistema di contrattazione collettiva, peraltro, si ricollega a quello dell'effettiva implementazione dei meccanismi di

174 Così, L. Corazza, La nuova nozione di appalto nel sistema delle tecniche di tutela del lavoratore, in WP CSDLE “Massimo D'Antona”.IT, 93/2009, p. 21. 
misurazione della rappresentatività delle organizzazioni dei lavoratori e dei datori di lavoro, atteso che gli ambiti di applicazione dei Ccnl costituiranno i perimetri all'interno dei quali misurare tale rappresentatività.

Il capitolo III, dedicato all'intervento della legge sul sistema di contrattazione collettiva, si porrà su un piano di ragionamento radicalmente diverso, per l'esperienza italiana di relazioni industriali. In un sistema, come il nostro, considerato a bassa istituzionalizzazione, l'idea stessa che in conseguenza dell'intervento eteronomo del legislatore vi possa essere un affievolimento dell'originaria libertà delle parti sociali di stabilire il campo di applicazione dei contratti collettivi è considerata dai più poco meno che eretica. Tuttavia, è noto come in questi ultimi anni sia riemersa una notevole attenzione, in ambienti sindacali ed accademici, ma anche nei luoghi della politica, per la questione dell'attuazione dell'art. 39 Cost.: in uno dei motivi ricorrenti della materia, le difficoltà crescenti che le parti sociali stanno incontrando nel governo del sistema di contrattazione collettiva hanno sollecitato un ritorno del dibattito sulla legge sindacale. In particolare, sono state oggetto di discussione alcune proposte di legge: una prima avanzata dalla Cgil (nel quadro della Carta dei diritti universali del lavoro) ed altre due elaborate da settori della dottrina (la proposta della rivista Diritti lavori mercati e la proposta del gruppo Freccia Rossa).

Nel complesso, tali progetti non potevano eludere il nodo dei meccanismi di definizione delle unità negoziali della contrattazione collettiva e della loro compatibilità con il principio di libertà sindacale. Questi svolgimenti hanno sollecitato un intenso dibattito, al quale la ricerca, col capitolo III, proverà a contribuire, con l'idea di fondo che quello dell'inquadramento per categorie sia, a ben guardare, un problema di soluzioni tecniche - se si vuole, di pesi e contrappesi - in grado di dare ordine al sistema contrattuale senza dimidiare oltre un certo limite la sfera di autonomia delle parti sociali. A tal fine, un rapido sguardo al diritto sindacale comparato consentirà di ampliare la riflessione sulla rosa di congegni tecnici adottabili, mentre una rassegna delle proposte di legge attuativa dell'art. 39 Cost. susseguitesi negli anni darà modo di verificare come siano cambiate, nel tempo, le sensibilità e gli approcci della riflessione giu- 
ridica italiana rispetto al tema de quo. In seguito, l'attenzione sarà posta sulle normative in materia di contrattazione collettiva del settore pubblico (d.lgs. n. 165/2001) e di limitazione al diritto di sciopero nei servizi pubblici essenziali (1. n. 146/1990), allo scopo di verificare, in questi due rami emblematici della disciplina gius-sindacale, quali equilibri siano stati trovati tra eteronomia ed autonomia nella delimitazione del campo di applicazione dei contratti collettivi.

Peraltro, oltre a sollecitare le parti sociali a rivedere i perimetri della contrattazione collettiva e a risvegliare l'interesse per l'emanazione di una legge sindacale, i processi di frammentazione del sistema contrattuale hanno reso più complessa, e meno scontata negli esiti, l'attività di selezione dei contratti collettivi da utilizzare quale parametro del trattamento economico e normativo dovuto ai lavoratori nei casi di rinvio all'autonomia collettiva. Ciò si è verificato specialmente nei casi di rinvio ai contratti collettivi in materia di calcolo del minimale contributivo, trattamento economico dovuto ai soci lavoratori di cooperativa e trattamento economico e normativo dovuto ai lavoratori impiegati nel contesto di contratti pubblici, oltre che di determinazione giurisprudenziale della retribuzione adeguata ex art. 36 Cost. Di ciò si occuperà il capitolo IV. Dopo un inquadramento per linee generali del tema dei rinvii all'autonomia collettiva per la definizione di un parametro del trattamento dovuto ai lavoratori, il capitolo si soffermerà sul problema della selezione dei contratti collettivi oggetto di rinvio. In particolare, la domanda che la dottrina oggi si pone e alla quale la giurisprudenza fatica, invero, a dare una risposta è la seguente: se le categorie non sono più definite con esattezza e gli ambiti di applicazione dei contratti collettivi si sovrappongono tra di loro, come si potrà individuare il contratto collettivo "della" categoria al quale fare riferimento come parametro del trattamento? 



\section{CAPitolo II}

\section{LA PROSPETTIVA INTERNA ALLE RELAZIONI INDUSTRIALI}

Sommario: 1. Le unità negoziali nella contrattazione collettiva del settore privato: considerazioni introduttive. -2 . Il problema del criterio di collegamento tra le unità negoziali e i rapporti di lavoro. - 3. La contrattazione collettiva di categoria al bivio. - 3.1. L'archivio Cnel dei contratti collettivi: cenni essenziali. - 3.2. Dal pluralismo al caos? - 3.2.1. Il grattacapo degli accordi "pirata". - 3.2.2. Il dumping contrattuale interno al sistema di rappresentanza "confederale”. - 4. Il percorso a ostacoli per una riorganizzazione del sistema contrattuale.

1. Le unità negoziali nella contrattazione collettiva del settore privato: considerazioni introduttive

In un sistema di contrattazione collettiva autoregolato dalle parti sociali e privo di un obbligo legale di portata generale di applicare i contratti collettivi, come quello del settore privato ${ }^{1}$, i problemi relativi alla definizione delle unità negoziali sono risolti all'interno delle relazioni industriali. L'unica garanzia operante è quella della libertà sindacale, che ricomprende sia la sfera di costituzione e di inquadramento dell'organizzazione sia quella della contrattazione collettiva. L'attività stessa con la quale le parti sociali definisco-

1 Per uno svolgimento della dottrina del contratto collettivo di diritto comune, M. Persiani, Il contratto collettivo di diritto comune nel sistema delle fonti del diritto del lavoro, in Arg. Dir. Lav., 2004, p. 13 ss. 
no le unità contrattuali è espressione diretta dell'art. 39, c. 1, Cost. Le parti sociali sono, in buona sostanza, "sovrane" nella definizione delle partizioni fondamentali della contrattazione collettiva: tali svolgimenti avvengono «spontaneamente nella realtà sindacale senza alcun piano preordinato dall'alto» ${ }^{2}$.

Per quanto tali partizioni siano anzitutto il risultato delle strutture del mercato ${ }^{3}$, è da un accordo tra le organizzazioni che stipulano i contratti collettivi che deriva l'individuazione delle situazioni di fatto per le quali si detta la regola comune di lavoro. La minore o maggiore ampiezza (più in generale, l'articolazione) di ogni unità contrattuale può considerarsi, perciò, l'esito dei compromessi raggiunti tra le organizzazioni contrapposte dei datori di lavoro e dei lavoratori. Per lo meno, in via di principio: non si può trascurare, infatti, come la definizione delle unità contrattuali sia condizionata da più complesse dinamiche sindacali ${ }^{4}$.

Vi possono influire conflitti di giurisdizione interni al movimento dei lavoratori, ad esempio per una contrapposizione tra sindacati di mestiere o professionali e sindacati industriali, oppure tra sindacati industriali con sfere di inquadramento adiacenti, che induca una delle due parti a desistere dal dettare una disciplina collettiva per un segmento della forza lavoro sul quale non abbia più la necessaria "presa rappresentativa". È un rilievo che rimanda ai primordi del movimento sindacale $^{5}$, ma che si è riproposto, con intensità crescente, a partire dagli anni Ottanta del secolo scorso, sia sul piano contrattuale ${ }^{6}$ sia su quello del conflitto collettivo ${ }^{7}$.

2 G. Pera, Problemi costituzionali del diritto sindacale italiano, cit., p. 55.

3 L'esistenza e la configurazione di un processo economico sono il presupposto del perché e del come vi si detti una disciplina collettiva: G.P. CELLA, Quali cambiamenti per le relazioni industriali italiane?, in Lav. Dir., 2007, p. 227.

4 Cfr. T. TREU, Regole e procedure nelle relazioni industriali: retaggi storici e criticità da affrontare, cit., passim.

5 Ricostruisce tali passaggi V. FoA, Cento anni di sindacato in Italia (1973), in ID., Per una storia del movimento operaio, Torino, Einaudi, 1980, spec. p. 107. In relazione alla questione delle unità contrattuali, cfr. G. GIUGNI, Bargaining units and labor organization in Italy, cit., p. 428.

6 Un cenno in P. LAmbertucci, Contratto collettivo, rappresentanza e rappresentatività sindacale: spunti per il dibattito, in Dir. Lav. Rel. Ind., 2009, p. 561.

7 G. PIno, Conflitto collettivo nei servizi pubblici essenziali, in Enc. Dir., Annali, X, 2017, p. 265 ss. 
Le implicazioni che derivano da contrapposizioni interne al mondo imprenditoriale non sono meno importanti ${ }^{8}$. Specialmente nei periodi caratterizzati da mutamenti degli assetti economici e dei processi produttivi e da trasformazioni tecnologiche, non è infrequente che l'apertura di nuove unità contrattuali si debba ai tentativi di una parte del tessuto imprenditoriale di sottrarsi a dinamiche negoziali reputate inadeguate rispetto alle aspettative di partecipazione al mercato. Data la tipica frammentazione, in Italia, del tessuto delle imprese manifatturiere, questo è stato spesso il caso delle contrapposizioni tra l'area di rappresentanza confindustriale e quella delle aziende artigiane e delle PMI: la dottrina lo rimarcava sin dai primi anni Sessanta del secolo scorso, ma oggi tali dinamiche si ripropongono con ben altro dinamismo ${ }^{9}$. Il terziario è un fronte forse persino più caldo, nel quale le prime operazioni manipolative delle aree contrattuali si osservavano già nei primi anni Novanta del secolo scorso ${ }^{10}$, ma che sembra ora diventato il centro più aggrovigliato della «giungla» ${ }^{11}$ della contrattazione collettiva ${ }^{12}$.

In situazioni di accentuato pluralismo organizzativo, non è da escludere che si pervenga, da parte dei gruppi, anche minoritari ${ }^{13}$, a una delimitazione opportunistica delle unità negoziali, in modo da sottrarsi all'applicazione di certe discipline collettive e forgiarne altre che si assumono più convenienti ${ }^{14}$. In un sistema volontaristico, sono le parti sociali a dover sorvegliare i propri spazi di regolazio-

8 Per un quadro complessivo, v. V. PAPA, L'attività sindacale delle organizzazioni datoriali. Rappresentanza, rappresentatività e contrattazione, Torino, Giappichelli, 2017.

9 V. PAPA, Verso una rappresentanza misurata? Strumenti e metodi di misurazione della rappresentatività datoriale, cit., p. 28.

10 P. Tosi, Le nuove tendenze del diritto del lavoro nel terziario, in Dir. Lav. Rel. Ind., 1991, pp. 627-631.

11 S. Ciucciovino, Mettere ordine nella giungla dei ccnl: un'esigenza indifferibile, in Dir. Lav. Rel. Ind., 2018, p. 227 ss.

12 G. Olini, I contratti nazionali: quanti sono e perché crescono, in Dir. Lav. Rel. Ind., 2016, p. 423 ma passim.

13 P. Bellocchi, Il contratto collettivo di diritto comune, in G. Proia (coord. da), Il diritto sindacale, in F. CARINCI (a cura di), Il lavoro subordinato, in M. BESSONE (dir. da), Trattato di diritto privato, Torino, Giappichelli, 2007, p. 277.

14 B. Balletti, Contributo alla teoria della autonomia sindacale, cit., pp. 247-248. 
ne ${ }^{15}$ dalle incursioni di altri, che mirino a dettare una disciplina diversa per le medesime attività produttive o per un segmento di es$\mathrm{se}^{16}$. Purché vi sia reciproco riconoscimento tra i soggetti che rappresentano le imprese e quelli che rappresentano i lavoratori, sarà possibile dar corso a un tentativo di inquadramento alternativo a quello invalso da più tempo nel settore ${ }^{17}$.

Riepilogando, l'esperienza delle relazioni industriali post-corporative si è caratterizzata per un sistema di definizione delle unità negoziali che potrebbe definirsi "implicito", ossia maturato in assenza di un accordo "esplicito" tra le parti sociali. Le categorie sono state individuate, in pratica, sulla base delle determinazioni che le organizzazioni stipulanti assumevano con i rinnovi dei Ccnl (confermandone o via via modificandone l'ambito di applicazione) o con l'apertura di nuove aree di negoziazione. Riprendendo su questo punto Mancini, potrebbe dirsi che le categorie sono il posterius non soltanto per l'ordinamento giuridico, dal quale non si ricava una nozione preconcetta di categoria, ma per lo stesso sistema contrattuale, dato che le parti sociali non si sono mai dotate di meccanismi di definizione ex ante delle sfere di influenza negoziale (né a maggior ragione di sistemi di risoluzione delle relative controversie).

Una diversa possibilità consisterebbe nella stipulazione di accordi "espliciti", tra le manifestazioni di vertice del sistema di relazioni industriali, che abbiano per oggetto le sfere di influenza negoziale o che provvedano, comunque, a stabilire alcune regole a tale proposito. Ragionando in astratto, ciò potrebbe intervenire all'interno del solo movimento dei lavoratori o, all'opposto, con una regolazione dei confini interna al sistema di rappresentanza delle imprese. In alternativa, le giurisdizioni contrattuali potreb-

15 Per questa nozione, v. R. MacKenzie, M. Martinez Lucio, The Realities of Regulatory Change: Beyond the Fetish of Deregulation, cit.

16 M. Grand, In difesa della rappresentanza sindacale, in Dir. Lav. Rel. Ind., 2004, p. 640. Vi si soffermano più di recente I. Alvino, L. ImBERTI, Contratto collettivo leader e rappresentanza datoriale, in Lab. Law Iss., 2018, 2, p. 12.

17 G. Pera, Note sui contratti collettivi «pirata», in Riv. It. Dir. Lav., 1997, I, p. 384, il quale osserva, però, che ogni tentativo di inquadramento alternativo a quello invalso fino ad un certo momento nel settore sarebbe destinato al fallimento, se non sorretto dal consenso della maggioranza delle imprese e dei lavoratori. 
bero stabilirsi congiuntamente, mediante accordi tra le maggiori confederazioni dei lavoratori e dei datori di lavoro o, comunque, in una sede di negoziato di vertice tra le parti sociali, anche dotata di stabilità, come ad esempio un organismo bilaterale costituito ad hoc. In ognuno di questi casi ne uscirebbe ovviamente affievolita (se non, in ipotesi, del tutto esclusa, a seconda della pervasività del negoziato di vertice sui perimetri) la tradizionale prerogativa delle federazioni di categoria di determinare esse stesse, in sede di contrattazione collettiva, i confini applicativi delle discipline collettive di lavoro.

È significativo che una parte della dottrina abbia attribuito portata e significato simili agli accordi interconfederali conclusi alcuni anni fa in materia di contrattazione collettiva nazionale e di misurazione della rappresentatività sindacale ${ }^{18}$. Posto che le previsioni sulla misurazione della rappresentatività delle organizzazioni stipulanti ai fini dello svolgimento della contrattazione collettiva nazionale presuppongono la "stabilità" dei perimetri contrattuali, gli accordi interconfederali si risolverebbero, anche senza averlo affermato apertamente, nell'attribuzione alle confederazioni della prerogativa di definire gli ambiti di applicazione dei contratti collettivi. Non sembra, cioè, che «possa avere più corso quella "autodeterminazione" della categoria contrattuale ad opera delle parti stipulanti il CCNL caratteristica dell'attuale produzione negoziale», atteso che, diversamente, «ci troveremmo di fronte ad un paradosso simile a quello del Barone di Münchhausen, il quale raccontava di essersi tratto in salvo da una palude tirandosi per i capelli ${ }^{19}$. Fuor di metafora, secondo tale orientamento il processo

18 V. PINTO, Gli accordi interconfederali del 2013 e i persistenti problemi teorici (e pratici) della definizione autonoma della "categoria contrattuale", in M. Barbera, A. Perulli (a cura di), Consenso, dissenso e rappresentanza: le nuove relazioni sindacali, Padova, Cedam, 2014, p. 361 ss. In particolare, l'autore si confrontava con il testo degli accordi conclusi nel 2013 in area confindustriale (Protocollo d'intesa del 31 maggio con Cgil, Cisl e Uil e successivi accordi con del 6 giugno con UGL, del 18 giugno con Cisal e del 4 luglio con Confsal), in area Confservizi (accordo del $1^{\circ}$ agosto con Cgil, Cisl e Uil) e nel settore cooperativo (accordo del 18 settembre tra Agci, Confcooperative e Legacoop con Cgil, Cisl e Uil).

19 Così, V. Pinto, Gli accordi interconfederali del 2013 e i persistenti problemi teorici (e pratici) della definizione autonoma della "categoria contrattuale", cit., pp. 367-368. 
di misurazione della rappresentatività delle organizzazioni che stipulano i contratti collettivi sottrarrebbe a queste ultime la prerogativa di modificarne il campo di applicazione, atteso che, così facendo, finirebbero per modificare anche il bacino di misurazione della rappresentatività, bloccando, di fatto, il funzionamento dell'intero meccanismo: «occorre ammettere che la competenza a dettare una definizione "autonoma" delle stesse sia orma transitata dal livello della categoria a quello interconfederale» ${ }^{20}$.

Questa tesi ha colto nel segno, nell'individuare, tra le righe degli accordi del 2013, le avvisaglie di un processo di complessiva riorganizzazione del sistema contrattuale, al termine del quale è presumibile che si avrà un riassestamento dei congegni di definizione delle categorie sperimentati da decenni nel modello italiano di relazioni industriali ${ }^{21}$.

Al contempo, essa si espone tuttavia ad alcune critiche incisive. Da una parte, si deve riconoscere che con gli accordi interconfederali citati (peraltro, il discorso non cambierebbe se si considerassero anche quelli più recenti: v. infra, § 4) sono state poste soltanto le premesse del processo di misurazione della rappresentatività delle organizzazioni che stipulano i Ccnl. Finché tale processo non sarà effettivamente implementato, pare quantomeno prematuro trarne delle conseguenze così incisive, se non proprio compromettenti, per le attività negoziali delle federazioni di categoria. Da un'altra, non è condivisibile l'idea stessa che un tale sovvertimento di precetti storicamente condivisi nel sistema sindacale di fatto

20 Ivi, p. 368.

21 Cfr. anche L. Bellardi, L'attuazione dell'accordo quadro: pluralità dei sistemi contrattuali ed eterogenesi dei fini. Alcune note di sintesi, in Dir. Lav. Rel. Ind., 2010, p. 387 ss. Alla luce delle disposizioni dell'accordo quadro del 22 gennaio 2009 e degli accordi interconfederali conclusi nell'anno successivo per i comparti dell'industria, dei servizi e dell'artigianato, l'autrice rilevava una complessiva tendenza alla ri-centralizzazione, in materia di cd. razionalizzazione del sistema contrattuale: una tendenza particolarmente evidente per il comparto artigiano, ove però è da sempre importante il livello interconfederale, ma non estranea alla stessa area di rappresentanza confindustriale (spec. accordo interconfederale Confindustria, Cisl e Uil 15 aprile 2009, n. 8 «Razionalizzazione e riduzione del numero dei contratti collettivi nazionali di lavoro di categoria») e di Confservizi (spec. accordo interconfederale Confservizi, Cisl e Uil 18 novembre 2009, n. 7 «Razionalizzazione e riduzione del numero dei contratti collettivi nazionali di lavoro di categoria»). 
sia effettuato in modo per così dire implicito. Per trasferire dalle federazioni di categoria alle confederazioni una tra le prerogative più «gelosamente difese» ${ }^{22}$ dalle organizzazioni sindacali occorrerebbe, infatti, un accordo che non soltanto affermi "apertamente" la novità rispetto al passato, ma che indichi, soprattutto, le soluzioni tecniche mediante le quali procedere, di lì in avanti, con la revisione, l'adattamento e la modifica delle unità negoziali: sarebbe questo probabilmente il cuore della questione ed il suo aspetto più controverso.

Del resto, in mancanza di un accordo particolarmente robusto su questi aspetti, anche sotto il profilo procedurale, le federazioni di categoria resterebbero libere di stabilire in autonomia le aree contrattuali, in base all'interpretazione dell'art. 39, c. 1, Cost. consolidata nella dottrina e nella giurisprudenza (ma prima ancora nella prassi contrattuale $)^{23}$. È una costante del modello italiano di relazioni industriali quella di un condizionamento più o meno intenso delle categorie sul sistema negoziale nel suo complesso, a «cominciare da quelle più forti che sono storicamente caratterizzate da orientamenti diversi» e che sono spesso in grado di mettere in discussione l'operatività delle «direttive provenienti dalle confederazioni ${ }^{24}$.

2. Il problema del criterio di collegamento tra le unità negoziali e i rapporti di lavoro

Quali che siano le soluzioni adottate dalle parti sociali per stabilire i perimetri negoziali, si pone, comunque, il problema se sia ricavabile dall'ordinamento giuridico una regola di (quantomeno tendenziale) coerenza tra le partizioni in unità negoziali della contratta-

22 A. Lassandari, Pluralità di contratti collettivi nazionali per la medesima categoria, cit., p. 291.

23 F. CARINCI, Una dichiarazione d'intenti: l'Accordo Quadro 22 gennaio 2009 sulla riforma degli assetti contrattuali, in Riv. It. Dir. Lav., 2009, I, pp. 194195.

24 T. TREU, Regole e procedure nelle relazioni industriali: retaggi storici e criticità da affrontare, cit., p. 11 . 
zione collettiva e l'individuazione dei contratti collettivi applicabili ai rapporti individuali di lavoro.

Secondo un orientamento consolidato da decenni nella giurisprudenza di legittimità, l'obbligatorietà dei contratti collettivi può derivare dall'affiliazione alle organizzazioni stipulanti ${ }^{25} \mathrm{o}$, in alternativa, dal rinvio esplicito o per fatti concludenti operato dalle parti individuali ad un determinato contratto collettivo o ad un sistema contrattuale nel suo complesso ${ }^{26}$. Ne deriva che per quanto possa essere considerato, per così dire, socialmente opportuno, che le discipline collettive siano rispettate dai soggetti che rientrano nel loro campo di applicazione, in assenza di una legge che ne preveda l'obbligatorietà, datori di lavoro e lavoratori sono liberi di applicare o meno un contratto collettivo.

In altri termini, il criterio per l'individuazione del contratto col-

25 Invero, che nel modello italiano si debba aver riguardo anzitutto o prevalentemente all'affiliazione sindacale del datore di lavoro, per l'applicazione di un contratto collettivo, è sostenuto in dottrina sin dagli anni Cinquanta del secolo scorso (cfr. G. Pera, Fondamento ed efficacia del contratto collettivo di diritto comune (1958), in Scritti di Giuseppe Pera, II, Diritto sindacale, Milano, Giuffrè, 2007, p. 989 ss.). Una volta obbligato, si ritiene che il datore di lavoro debba applicare generalmente il contratto collettivo ai propri dipendenti. Che la sottovalutazione della posizione giuridica e sociale del lavoratore, che da ciò in qualche modo ne deriva sia, sotto più d'un aspetto, criticabile, diverrà chiaro con il prorompere della legislazione dell'emergenza e con la diffusione di una contrattazione collettiva non più necessariamente (o non per tutti) acquisitiva. Questi svolgimenti sono, com'è ovvio, più evidenti per il livello aziendale di contrattazione collettiva, ove il problema dell'efficacia soggettiva degli accordi rispetto ai lavoratori dissenzienti e non iscritti ai sindacati stipulanti è avvertito con più urgenza: è a questo proposito, infatti, che la giurisprudenza di legittimità ha dovuto precisare il tiro rispetto ai propri passati indirizzi e che il legislatore è intervenuto, alcuni anni fa, con la manovra di ferragosto del 2011 (V. BAVAro, Azienda contratto sindacato, Bari, Cacucci, 2012).

26 L'orientamento secondo il quale i contratti collettivi di diritto comune obbligano i soggetti iscritti alle associazioni stipulanti o quelli che li abbiano esplicitamente o implicitamente adottati si era consolidato, con isolate voci contrarie, già nei primi anni Cinquanta del secolo scorso (cfr. L. Mengoni, Le fonti del diritto del lavoro in Italia, in G. Bold et al., Le fonti del diritto del lavoro, Lussemburgo, Comunità europea del carbone e dell'acciaio Alta autorità, 1962, pp. 149-152). Di recente, tra le molte, v. Cass. 2 maggio 2019, n. 11537, in RGL News, 2019, 3, p. 8 ss. Per una trattazione di respiro: C. Zoli, Contratto collettivo come fonte e contrattazione collettiva come sistema di produzione di regole, in M. PERSIANI (a cura di), Le fonti del diritto del lavoro, in F. Carinci, M. Persiani (dir. da), Trattato di diritto del lavoro, Padova, Cedam, 2010, p. 487 ss. 
lettivo da applicare al rapporto di lavoro è quello $\mathrm{cd}$. soggettivo ${ }^{27}$, che si risolve in una quaestio voluntatis ${ }^{28}$. Più che come legge professionale della categoria, il contratto collettivo si configura, in questo modello, come uno «schema di disciplina nell'interesse di coloro che vogliono servirsene» ${ }^{29}$.

Comunque, qualora per affiliazione sindacale o previo rinvio individuale un contratto collettivo possa considerarsi applicabile al rapporto di lavoro, parrebbe di primo acchito scontato che tale contratto sia quello stipulato per disciplinare proprio quella tipologia di attività economica e di lavoro. In altri termini, il contratto collettivo applicato sarà, in linea di massima, quello che nel campo di applicazione ricomprenda le situazioni di fatto dedotte nel contratto di lavoro. In modo tale, cioè, che vi sia una corrispondenza tra le discipline collettive applicate ai rapporti di lavoro e le partizioni fondamentali del sistema di contrattazione collettiva. Oltre che ad una comprensibile logica organizzativa delle relazioni sindacali, ciò risponderebbe, a ben vedere, a un'esigenza pratica, che si può sintetizzare in un interrogativo: in quale testo contrattuale dovrebbero rinvenirsi una descrizione delle mansioni, un trattamento economico e una disciplina del lavoro adeguate, se non in quello che si propone di regolare precisamente quel tipo di situazioni di fatto? Tuttavia, occorre riconoscere che questo non è necessariamente il caso, in assenza di una legge che disponga in tal senso: la «normalità» ${ }^{30}$ può non essere la regola, in un settore dell'ordinamento che rinviene il proprio presupposto nei rapporti di forza.

Anche nel caso in cui un contratto collettivo sia applicabile al rapporto di lavoro, a determinare quale non sarà un collegamento (criterio) "oggettivo", tra il tipo di attività esercitata dal datore di lavoro e/o dal lavoratore ed il campo di applicazione dell'accordo. Ad essere determinante sarà l'affiliazione sindacale oppure l'interpretazione della manifestazione di volontà dalle quale si trae l'intento

27 L. Riva Sanseverino, Contratto collettivo di lavoro, cit., passim.

28 Sul tema, v. A. Vallebona, Autonomia collettiva e occupazione: l'efficacia soggettiva del contratto collettivo, in Dir. Lav. Rel. Ind., 1997, p. 381 ss.

29 Così, P. Bellocchi, Il contratto collettivo di diritto comune, cit., p. 280.

30 Per una brillante trattazione del tema v. W. BigiavI, «Normalità»e «anormalità» nella costruzione giuridica, in Riv. Dir. Civ., 1968, I, p. 518 ss. 
delle parti di applicare il contratto collettivo ${ }^{31}$. Come affermato dalle Sezioni Unite con la storica sentenza n. 2665/1997 sull'applicazione dell'art. 2070 c.c. ai contratti collettivi di diritto comune ${ }^{32}$, «dai vigenti principi di libertà contrattuale e di associazione sindacale può derivare [...] l'eventualità che al rapporto individuale di lavoro si applichi un contratto del tutto innaturale rispetto alle oggettive caratteristiche dell'impresa». Da qui, il principio di diritto, confermato dalla giurisprudenza successiva ${ }^{33}$ : nell'ipotesi di contratto di lavoro regolato dal contratto collettivo di diritto comune proprio di un settore non corrispondente a quello dell'attività svolta dall'imprenditore, il lavoratore non può aspirare all'applicazione di un contratto collettivo diverso ${ }^{34}$.

$\mathrm{Si}$ potrebbe persino osservare che, in un sistema sindacale autoregolato dalle parti sociali, la definizione del campo di applicazione dei contratti collettivi abbia, in via di principio, una modesta "rilevanza giuridica", per lo meno «se i soggetti - identificati nella loro generalità dalla manifestazione di autonomia sindacale non aderiscono alla formazione collettiva per tutelare gli interessi tipici professionali indicati nella tipica autoregolamentazione del sindacato» ${ }^{35}$. È come dire, se si preferisce, che soltanto coloro che abbiano concorso, anche indirettamente, tramite le proprie associazioni, alla formazione della disciplina collettiva, o che la abbiano in alternativa recepita con un atto di volontà nei rapporti di lavoro, vi saranno soggetti, quale che ne sia il potenziale bacino sociale di applicazione ${ }^{36}$.

31 Cfr., per uno svolgimento più compiuto di questioni che con la sintetica formulazione di cui al testo si sono soltanto evocate, A. Lo FAro, Contratto collettivo (lavoro privato), in Enc. Dir., Annali, VI, 2013, p. 204.

32 Cass. SSUU n. 2665/1997, cit. Cfr. O. MazzotTa, Intervento, in AA.VV., Categorie professionali e contratti collettivi, cit., pp. 97-100 ma spec. 99.

33 Tra le molte, Cass. n. 11372/2008, cit., Cass. n. 16340/2009, cit., Cass. n. 26742/2014, cit.

34 G. Proia, M. Gambacciani, Il contratto collettivo di diritto comune, in G. Proia (a cura di), Organizzazione sindacale e contrattazione collettiva, cit., pp. 657-658.

35 Così, B. Balletti, Contributo alla teoria della autonomia sindacale, cit., p. 247.

36 G. Vardaro, Il mutamento della funzione del contratto collettivo, in Dir. Lav. Rel. Ind., 1983, pp. 741-742. 
Beninteso, non si vuole con ciò negare che il sistema di relazioni industriali sia pregiudicato dai datori di lavoro che restino, per così dire, fuori dal sistema contrattuale o che, pur essendovi ricompresi, si sottraggano all'applicazione del contratto collettivo "naturale", per adottarne uno che sia, ad esempio, meno oneroso nelle retribuzioni oppure più rigido nella disciplina del personale.

Tuttavia, è nell'ordine delle cose di un sistema privo di una legge che, in queste situazioni, la reazione non possa che consistere negli atti di pressione che le organizzazioni dei datori di lavoro e dei lavoratori siano in grado di attuare, in base al contesto produttivo ed alla fase storica che si attraversa ${ }^{37}$ : oltre ad una razionalizzazione dei tavoli e dei perimetri contrattuali, è lo sciopero (per le organizzazioni dei lavoratori) lo strumento cui ricorrere per attrarre nella sfera di applicazione del contratto collettivo tutti i soggetti in essa astrattamente ricompresi ${ }^{38}$. Le complicazioni interne ai meccanismi di ripartizione del sistema contrattuale in unità negoziali sono, cioè, assorbite dalle dinamiche negoziali tra le parti sociali e in ultima analisi dal conflitto collettivo ${ }^{39}$. L'intera questione è «affidata alla capacità dei sindacati di far rispettare $\mathrm{i}$ contratti» ${ }^{40}$.

37 Cfr. M.G. Garofalo, Per una teoria giuridica del contratto collettivo. Qualche osservazione di metodo, in Dir. Lav. Rel. Ind., 2011, p. 515 ss.

38 G. VARDARO, Il mutamento della funzione del contratto collettivo, cit., p. 742. Lo sciopero cd. giurisdizionale può considerarsi uno strumento di reazione verso comportamenti consistenti nello shopping contrattuale: diffuso soprattutto in altri sistemi di relazioni industriali (ad esempio, in alcune esperienze nordiche), tale manifestazione di conflitto collettivo è più efficace se attuata con l'appoggio di gruppi di lavoratori da principio estranei alla contesa, i quali, per solidarietà o, come si dice, "per simpatia", si uniscano alla lotta principale, auspicandone e favorendone l'esito positivo (G. Pera, Problemi costituzionali del diritto sindacale italiano, cit., p. 55).

39 M. BARBIERI, Intervento, in Autonomia collettiva e occupazione. Atti del XII Congresso nazionale di diritto del lavoro Milano, 23-25 maggio 1997, cit., p. 179.

40 P. Bellocchi, Il contratto collettivo di diritto comune, cit., p. 281. 
3. La contrattazione collettiva di categoria al bivio

Riepilogando, in un modello di contrattazione collettiva autoregolato mediante accordi tra le parti sociali, gli svolgimenti inerenti alla definizione delle unità negoziali sono un affare interno al sistema di relazioni industriali, che viene regolato per via delle dinamiche sindacali, dei rapporti di forza e del conflitto collettivo. La modifica del campo di applicazione dei contratti collettivi o l'apertura di nuove aree contrattuali ha come unico requisito il reciproco riconoscimento tra le organizzazioni contrapposte, ossia - per esser chiari - il fatto in sé che sia stipulato un contratto collettivo (dato che la stipulazione presuppone logicamente che ne sia stato individuato il campo di applicazione). Esito di questi svolgimenti è un sistema contrattuale particolarmente agile, che senza ingessature o rallentamenti può adattare le proprie unità di negoziazione ai mutamenti del mercato e della forza lavoro, come avvenne negli anni Sessanta del secolo scorso con il prorompere della contrattazione articolata.

È anche vero che in situazioni di accentuato pluralismo - dal lato dei lavoratori e dal lato dei datori di lavoro, come nel sistema italiano di questi anni - possono essere messe in discussione facilmente le tradizionali sfere di giurisdizione negoziale, senza che ne derivi in tempi rapidi un riassestamento. Il che può finire per creare una situazione di "caos", sia per le parti sociali sia per gli operatori economici, dato che non sono ricavabili dall'ordinamento giuridico, a rigore, strumenti per mezzo dei quali orientare con esattezza le determinazioni delle parti dei rapporti di lavoro circa i contratti collettivi cui dare applicazione, soprattutto se sono stipulati molteplici contratti collettivi con ambito di applicazione in parte coincidente. Ebbene, dopo aver posto le basi del ragionamento giova cominciare ad interrogarsi su quale sia, per il tema che ci occupa, lo "stato dell'arte" del sistema di contrattazione collettiva del settore privato. 


\subsection{L'archivio Cnel dei contratti collettivi: cenni essenziali}

Il punto di partenza non può che essere costituito da quanto si desume dalla banca dati pubblica tenuta dal Cnel («Archivio nazionale dei contratti collettivi di lavoro»). L'archivio è istituito ai sensi dell'art. 17, 1. n. 936/1986, al fine di consentire il deposito dei contratti e degli accordi collettivi di lavoro e di agevolarne il reperimento e la consultazione; la legge prevede che i criteri di conservazione e di catalogazione dei contratti collettivi siano stabiliti di concerto con il Ministero del lavoro e delle politiche sociali e con il centro elettronico di documentazione della Corte di cassazione, previa consultazione delle organizzazioni sindacali dei lavoratori e dei datori di lavoro ${ }^{41}$. Direttive per l'organizzazione dell'archivio sono impartite (art. 16, 1. n. 936/1986) da una «Commissione informazione e lavoro» (questa è la sua attuale denominazione), presieduta dal Presidente del Cnel e nella quale sono rappresentate le parti sociali. Nel corso dell'attuale Consiliatura sono stati effettuati rilevantissimi lavori di aggiornamento e di ammodernamento della banca dati dei contratti collettivi, che costituisce ormai una fonte ricca di dati, informazioni ed elaborazioni sul sistema contrattuale, sia privato sia pubblico (è quasi superfluo rimarcare che ciò costituisce un'impor-

41 Si veda il sito www.cnel.it/Archivio-Contratti (consultato l'ultima volta il 30 aprile 2020). L'art. 17, cit., dispone ai suoi primi tre commi: «1. È istituito presso il CNEL l'archivio nazionale dei contratti e degli accordi collettivi di lavoro presso il quale vengono depositati in copia autentica gli accordi di rinnovo e i nuovi contratti entro 30 giorni dalla loro stipula e dalla loro stesura. 2. Il deposito avviene a cura dei soggetti stipulanti. 3. L'organizzazione dell'archivio nazionale dei contratti e degli accordi collettivi di lavoro deve consentire la loro conservazione nel tempo e la pubblica consultazione. [...]». Il Cnel ha di recente esercitato il suo potere di iniziativa legislativa, approvando (seduta del 27 marzo 2019) un disegno di legge ai sensi dell'art. 99, c. 3, Cost., volto ad ampliare e precisare le prerogative del Consiglio in questa materia. In particolare, il d.d.l. S. 1232, comunicato alla Presidenza del Senato il 5 aprile 2019, nel suo unico art. 1, prevede l'attribuzione al Cnel in collaborazione con l'Inps della prerogativa di definire un «codice unico di identificazione dei contratti e degli accordi collettivi di lavoro nazionali», mediante una sequenza alfanumerica (c. 1). Ciò dovrebbe consentire di armonizzare gli archivi del Cnel (alimentato dai depositi effettuati dalle parti sociali) e dell'Inps (alimentato dai flussi Uniemens derivanti dalle denunce dei datori di lavoro) e di agevolare, in definitiva, le verifiche relative all'applicazione dei contratti collettivi da parte dei datori di lavoro. 
tante novità per gli studi sulla contrattazione collettiva e, in prospettiva, per gli andamenti del sistema contrattuale).

Attualmente, la ricerca dei Ccnl all'interno dell'archivio «corrente» dei contratti collettivi «in vigore» ${ }^{42}$ avviene su due livellii3 ${ }^{43}$. Una prima catalogazione è effettuata sulla base di 14 macrosettori contrattuali, definiti con le parti sociali rappresentate in seno alla Commissione istituita ai sensi dell'art. 16, cit. Tali macrosettori dovrebbero grosso modo corrispondere ad alcune partizioni basilari dell'economia, per come individuate dagli attori delle relazioni industriali: agricoltura, alimentaristi-agroindustriale, altri vari, amministrazione pubblica, aziende di servizi, chimici, commercio, credito assicurazioni, edilizia, enti e istituzioni private, meccanici, poligrafici e spettacolo, tessili, trasporti (in ordine alfabetico).

Il secondo livello di ricerca nell'archivio è costituito dalla "categoria”. Ogni contratto collettivo, individuando un campo di applicazione, genera, con la registrazione nell'archivio pubblico, la propria categoria, all'interno di uno dei macrosettori citati. La determinazione delle parti sociali in ordine a tale definizione è meramente recepita dal Cnel: la nozione di categoria adottata dall'archivio pubblico coincide, in sostanza, con il campo di applicazione dei contratti collettivi che vi sono depositati (ma non sarebbe pensabile il contrario, ossia un rimaneggiamento delle determinazioni adottate dalle parti sociali).

Peraltro, il Cnel sta predisponendo in questi anni sistemi più avanzati di ricerca. Giovandosi di collaborazioni interistituzionali,

42 Traggo da CNel, Notiziario dell'Archivio contratti, n. 30, nuova serie, luglio 2018, p. 3: «sono considerati correnti i CCNL non dichiarati cessati o confluiti mediante una comunicazione dei contraenti all'ufficio, oppure mediante una dichiarazione contenuta in un altro CCNL che li sostituisce». Si badi che i Ccnl correnti non sono costituiti soltanto da "testi definitivi", bensì, più spesso, da una serie di accordi che si sovrappongono al testo vero e proprio del Ccnl al fine di regolarne aspetti «specifici, o che nel tempo hanno rinnovato solo alcuni istituti» (così, CNEL, Notiziario dell'Archivio contratti, n. 33, nuova serie, novembre 2019, pp. 10-11). In particolare, gli accordi depositati sono catalogati come $i$ ) testo definitivo, ii) accordo di rinnovo, iii) accordo economico, $i v$ ) verbale integrativo.

43 Le modalità di ricerca all'interno dell'archivio pubblico sono in questi anni in via di ripensamento, al fine di agevolare ulteriormente il reperimento e la consultazione dei testi contrattuali (v. www.cnel.it/Archivio-Contratti, consultato l'ultima volta il 30 aprile 2020, in particolare la sezione guida alla consultazione, aggiornamento al 10 febbraio 2020). 
sono in via di elaborazione mappature più progredite del sistema contrattuale ${ }^{44}$. Come ad esempio quelle che stabiliscono un collegamento tra i codici attribuiti ai contratti collettivi dal Cnel (codici associati ai depositi delle parti sociali) e dall'Inps (codici associati ai sistemi di comunicazioni effettuate dai datori di lavoro all'istituto): tali operazioni rappresentano il presupposto per conoscere con esattezza il numero di imprese e di lavoratori ai quali sono applicati i contratti collettivi. Altro collegamento importante, in via di implementazione, è quello tra i codici Cnel dei contratti collettivi e i codici Ateco delle attività economiche, allo scopo di individuare con esattezza i perimetri dei Ccnl, in relazione alle attività economiche ricomprese nei loro campi di applicazione, nonché per mettere in relazione l'archivio Cnel dei contratti con le banche dati Istat $\mathrm{e}$ Unioncamere. Se tali informazioni riguardano i Ccnl vigenti, è rilevante che l'archivio pubblico dei contratti consenta altresì l'accesso ai Ccnl "confluiti" ed a quelli "cessati", che sono utili soprattutto per ricostruire l'evoluzione storica delle discipline negoziali nei diversi settori contrattuali.

Il Cnel pubblica con cadenza periodica una serie di utilissimi report sulla contrattazione collettiva, dai quali sono reperibili dati e spunti di riflessione sulle dinamiche del sistema contrattuale ${ }^{45}$.

\subsection{Dal pluralismo al caos?}

Esito di un tessuto economico-produttivo complesso, diversamente articolato su base territoriale e soggetto a frequenti trasformazioni, il modello italiano di relazioni sindacali è caratterizzato da un elevato livello di pluralismo organizzativo, sia dal lato dei lavoratori sia da quello dei datori di lavoro, che si riflette giocoforza nella stipulazione di alcune centinaia di $\mathrm{Ccnl}^{46}$.

44 Qualche cenno da parte di M. Ricci, L'accordo interconfederale del 9 marzo 2018: una svolta dagli esiti incerti, cit., p. 1399, ma passim.

45 L'ultimo in ordine di tempo è CNeL, $10^{\circ}$ Report periodico dei Contratti Collettivi Nazionali di Lavoro depositati nell'archivio CNEL, aggiornamento dicembre 2019.

46 In particolare, sull'influenza che il sistema di rappresentanza delle imprese esercita sulla struttura della contrattazione collettiva, v. G.P. Cella, T. Treu, Relazioni industriali e contrattazione collettiva, cit., p. 117. 
Per non riprendere che alcuni elementi di contesto, si consideri, per prima cosa, che in molti settori la frammentazione del sistema di rappresentanza delle imprese porta ad alcune diversificazioni, talora minimali, talaltra più accentuate, delle discipline di categoria, in base alla natura industriale o artigiana delle aziende, alla condizione (ambigua, anche nell'inquadramento contrattuale) ${ }^{47}$ di PMI o alla forma societaria (cooperative o consorzi).

Peraltro, nonostante non siano mancati alcuni segnali di segno contrario, la competizione interna al sistema di rappresentanza delle imprese è aumentata da alcuni anni a questa parte. Vi sono casi in cui una stessa coalizione di organizzazioni sindacali di categoria è indotta a stipulare Ccnl simili, se non analoghi, con diverse organizzazioni datoriali ${ }^{48}$. In altri la frammentazione della rappresentanza datoriale determina, invece, uno scompaginamento nel fronte opposto, delle organizzazioni dei lavoratori ${ }^{49}$.

Processi interni al mondo imprenditoriale hanno anche portato alla conclusione di accordi che, in virtù di una scelta di politica contrattuale, creano una nuova unità "per secessione" da quella in cui era in passato ricompresa: è il caso della lunga vertenza che ha portato nel 2018 alla stipulazione del Ccnl della Distribuzione Moderna Organizzata ${ }^{50}$. Sono vicende che talora suscitano clamore, come nel caso del distacco del gruppo FCA dal sistema di rappresentanza di Federmeccanica, che aveva lo scopo di realizzare un sistema contrattuale alternativo alla categoria dei metalmeccanici industria, in

47 T. TREU, Regole e procedure nelle relazioni industriali: retaggi storici e criticità da affrontare, cit., p. 15, nota 16 .

48 D. GotTARDI, La contrattazione collettiva tra destrutturazione e ri-regolazione, in Lav. Dir., 2016, p. 886, con riferimento alle agenzie di somministrazione di lavoro, riportava l'esempio dei Ccnl stipulati da Assolavoro da un lato (27 febbraio 2014) e da Assosomm dall'altro lato (7 aprile 2014), sempre con Cgil, Cisl, Uil, Felsa-Cisl, Nidil-Cgil e Uil-Temp.

49 Cfr. P. Tomassetti, La nozione di sindacato comparativamente più rappresentativo nel decreto legislativo n. 81/2015, in Dir. Rel. Ind., 2016, pp. 380-381 testo e nota 55, con riferimento al settore della panificazione, nel quale a partire dal 2012-2013 Assipan, Fippa e Fiesa hanno rotto l'unità del settore, stipulando, la prima, il contratto collettivo con Ugl Terziario (19 giugno 2013, ma ora rinnovo del 4 aprile 2018) e le altre due con le organizzazioni di categoria Flai-Cgil, Fai-Cisl e Uila-Uil (13 febbraio 2013, ma ora rinnovo del 17 maggio 2017).

50 Ccnl 19 dicembre 2018 stipulato da Federdistribuzione e Filcams-Cgil, Fisascat-Cisl, Uiltucs-Uil. 
cui l'automotive era tradizionalmente ricompreso. Più in generale, non è infrequente che, per imprese in grado di sostenerne il peso, in termini economico-organizzativi, la contrattazione collettiva si svolga sì al livello aziendale o di gruppo, ma dando luogo a testi di una complessità normativa e rivolti ad una platea di lavoratori tale, da apparire nei fatti come contratti di categoria ${ }^{51}$.

Si tenga presente, inoltre, che, in virtù di scelte di politica sindacale talvolta risalenti nel tempo, il sistema negoziale comprende anche Ccnl applicati a poche migliaia di lavoratori, dipendenti, nel complesso, da alcune centinaia (talvolta decine) di aziende ${ }^{52}$.

Anche la frammentazione interna al movimento sindacale dei lavoratori induce fenomeni di proliferazione contrattuale: più che $i$ casi della cd. contrattazione separata degli anni Duemila (in specie, nel metalmeccanico e nel commercio-terziario) ${ }^{53}$, si hanno in mente quelli dovuti alla risalente conventio ad excludendum del sistema sindacale di fatto ${ }^{54}$, che in molti settori tiene fuori l'Ugl dai tavoli ne-

51 È un elemento, presente anche in altri sistemi di relazioni industriali, che l'esperienza italiana conosce da tempo: ne trattava già L. MENGONI, Il regime giuridico delle organizzazioni professionali in Italia, cit., p. 450, a proposito dei contratti stipulati dall'ENI, «in rappresentanza di singole aziende del gruppo» (prima della costituzione dell'ASAP e della fuoriuscita dal sistema di rappresentanza confindustriale).

52 Si prenda il Ccnl per gli addetti alle aziende produttrici di penne, matite, parti staccate di matite, penne, articoli affini e per gli addetti alle aziende produttrici di spazzole, pennelli, scope e preparatrici relative materie prime, stipulato tra Assoscrittura, Assospazzole, Femca-Cisl, Filctem-Cgil, Uiltec-Uil, che è applicato, secondo quanto si desume dal Database Cnel-Inps, a 538 addetti, dipendenti da 19 aziende. Si tenga presente, per avere un'unità di misura del bacino di alcuni tra i maggiori Ccnl, che, stando al medesimo database pubblico, il Ccnl per i dipendenti dalle aziende metalmeccaniche e della installazione di impianti, stipulato tra Federmeccanica, Assistal, Fim-Cisl, Fiom-Cgil, Uilm-Uil, risulta applicato a 1.445.293 addetti, dipendenti da 57.314 aziende, mentre il Ccnl Industria chimica, chimica-farmaceutica, stipulato tra Federchimica, Farmindustria, Filctem-Cgil, Femca-Cisl, Uiltec-Uil, risulta applicato a 209.302 addetti, dipendenti da 3.832 aziende.

53 A. MARESCA, Accordi collettivi separati: tra libertà contrattuale e democrazia sindacale, in Riv. It. Dir. Lav., 2010, I, p. 29 ss.

54 V. G. Mazzoni, L'azione sindacale e lo Statuto dei lavoratori, Milano, Giuffrè, 1974, pp. 98-99, e più di recente D. GOTTARDI, La contrattazione collettiva tra destrutturazione e ri-regolazione, cit., p. 886, ma passim. 
goziali di Cgil, Cisl e Uil per la contrattazione di categoria ${ }^{55}$. Esiste, inoltre, per i dirigenti, un'ampia struttura negoziale, che in alcuni casi supera la logica della contrattazione verticale o per ramo d'industria $^{56}$, mentre in altri si muove in parallelo a quella per le altre categorie di lavoratori dipendenti ${ }^{57}$. Con l'accresciuta articolazione del mercato del lavoro, ha acquisito più rilevanza anche l'esperienza della contrattazione di mestiere o professionale ${ }^{58}$.

Da queste brevissime considerazioni si ricava l'indicazione di massima di un sistema contrattuale caratterizzato da un elevato livello di pluralismo, e la cui complessità è con il tempo aumentata, con la maggiore articolazione della forza lavoro e con le trasformazioni tecnologiche e dei processi produttivi.

Ora, per avere un metro di paragone del numero di Ccnl da considerare proporzionato (con tutte le approssimazioni del caso) alle caratteristiche del sistema italiano di relazioni industriali, si consideri che nel documento unitario di Cgil, Cisl e Uil del maggio 2008, recante «Linee di riforma della struttura della contrattazione», era valutato eccessivo il numero di «oltre $400 \mathrm{Ccnl}$ » allora presenti: se ne auspicava infatti una «razionalizzazione», per via di una «verifica [...] delle aree di copertura» e di «accorpamenti per aree omogenee e per settori, favorendo la riunificazione di contratti analoghi facenti riferimento a diverse organizzazioni di rappresentanza datoriale». Iniziava tuttavia in quegli anni la crisi economica. Otto anni dopo (nel 2016) il numero di Ccnl depositati nell'archivio Cnel superava le 700 unità $^{59}$ e dopo altri tre anni sarebbe stata superata quota

55 Un esempio di tali svolgimenti è rappresentato dal Ccnl Agricoltura operai agricoli e florovivaisti, che è stipulato da Confagricoltura, Coldiretti e Cia, con Flai-Cgil, Fai-Cisl e Uila-Uil da un lato (19 giugno 2018) e con Sindacato nazionale agricoli e forestali Ugl dall'altro lato (16 giugno 2016).

56 Esempio rilevante di contrattazione collettiva "orizzontale" per i dirigenti è il Ccnl per i Dirigenti di aziende industriali tra Confindustria e Federmanager (30 dicembre 2014).

57 È il caso del contratto collettivo Bancari ABI Dirigenti (13 luglio 2015), tra ABI, FABI, First-Cisl, Fisac-Cgil, Sinfub, Ugl credito, Uilca-Uil e Unisin; oppure, si pensi al contratto collettivo Autoferrotranvieri Dirigenti (27 aprile 2010), tra Asstra e Federmanager.

58 Cfr. P. Bellocchi, La libertà sindacale, cit., p. 39.

59 Cnel, $3^{\circ}$ Report periodico dei Contratti Collettivi Nazionali di Lavoro depositati nell'archivio CNEL, aggiornamento marzo 2016. 
$900^{60}$ : una vera e propria «esplosione» negoziale ${ }^{61}$. Occorre allora soffermarsi sulle ragioni che sembrano aver esasperato, da alcuni anni, il tipico carattere pluralistico del modello italiano, finendo per renderlo caotico e difficilmente governabile da parte dei suoi attori più accreditati ${ }^{62}$.

\subsubsection{Il grattacapo degli accordi "pirata"}

Il numero complessivo di Ccnl depositati nell'archivio Cnel ha subito un'inflazione radicale tra il 2008 (400), il 2013 (600) e il 2019 (900). Il macrosettore nel quale la crescita in numero assoluto è stata maggiore è il commercio: i $121 \mathrm{Ccnl}$ del 2013 erano diventati 226 nel $2018^{63}$. Secondo i dati risalenti a dicembre $2019^{64}$, il numero di Ccnl depositati nell'archivio dei contratti vigenti ${ }^{65}$ era di 922 ,

\footnotetext{
${ }^{60} \mathrm{CNEL}, 10^{\circ}$ Report periodico dei Contratti Collettivi Nazionali di Lavoro depositati nell'archivio CNEL, cit.

${ }_{61}$ Così, G. Olini, I contratti nazionali: quanti sono e perché crescono, cit., p. 419.

62 Il rilievo è condiviso da larghi settori della dottrina: cfr. tra i molti L. MARIUCCI, Gli eterni ritorni: dentro, fuori o oltre l'art. 39 della Costituzione?, in L. Zoppoli, A. Zoppoli, M. Delfino (a cura di), Una nuova Costituzione per il sistema di relazioni sindacali?, Napoli, Editoriale scientifica, 2014, p. 491, L. BELLARDI, Relazioni industriali e contrattazione collettiva: criticità e prospettive, in Lav. Dir., 2016, p. 939, I. Alvino, L. Imberti, Contratto collettivo leader e rappresentanza datoriale, cit., p. 13.

63 CNeL, Notiziario dell'Archivio contratti, n. 30, cit., pp. 8-9. In quel periodo l'unica decrescita si è registrata per la PA (da 40 a 20), come era lecito attendersi, con i rinnovi successivi al d.lgs. n. 150/2009, che aveva disposto la riduzione a 4 del numero dei comparti e delle aree dirigenziali, ed al Ccnq del 13 settembre 2016, che ha dato seguito a tale previsione.

64 CNel, $10^{\circ}$ Report periodico dei Contratti Collettivi Nazionali di Lavoro depositati nell'archivio CNEL, cit.

65 Si tenga presente che il deposito dei contratti collettivi nell'archivio Cnel non è obbligatorio per le parti stipulanti (meglio: dal mancato deposito non derivano conseguenze particolari, ad esempio in ordine alla astratta applicabilità ai rapporti di lavoro dei $\mathrm{Ccnl}$ ). Al costo di una certa approssimazione, si può comunque ritenere che il numero di Ccnl depositati sia sostanzialmente identico a quello dei Ccnl "esistenti", atteso che il deposito presso l'archivio è una misura minima, non impegnativa per i soggetti stipulanti e che anzi consente loro di "accreditarsi" come soggetti effettivamente attivi nel sistema contrattuale; non vi sarebbe motivo, perciò, di stipulare un Ccnl per poi non depositarlo in archivio (benché possano certamente esservi eccezioni a questa osservazione: potrebbe essere il caso, per
} 
così ripartiti tra i macrosettori: 53 agricoltura, 33 chimici, 36 meccanici, 29 tessili, 42 alimentaristi-agroindustriale, 75 edilizia, 43 poligrafici e spettacolo, 244 commercio, 70 trasporti, 28 credito e assicurazioni, 47 aziende di servizi, 19 amministrazione pubblica, 114 enti e istituzioni private, 89 altri vari.

Una tale proliferazione di Ccnl si deve per lo più ad accordi stipulati da organizzazioni datoriali e/o dei lavoratori estranee ai principali circuiti della rappresentanza di interessi nel Paese.

Il problema della stipulazione di contratti collettivi alternativi a quelli del sistema confederale da parte di soggetti newcomers (considerati) scarsamente rappresentativi emergeva già nella metà degli anni Novanta del secolo scorso ${ }^{66}$. Si trattava di contratti collettivi che prevedevano un trattamento economico e normativo inferiore a quello dei contratti collettivi del sistema confederale. In alcuni casi erano state ravvisate clausole negoziali apertamente contra legem ${ }^{67}$. Per contrastare le ricadute del fenomeno sull'approvvigionamento finanziario del sistema previdenziale ${ }^{68}$, il legislatore (art. 2, c. 25, 1. n. 549/1995, di interpretazione autentica dell'art. 1, c. 1, d.1. n. 338/1989, conv. con modifiche 1 . n. 389/1989) stabiliva che, in caso di pluralità di contratti collettivi con ambito di applicazione sovrapposto, la retribuzione base per il calcolo del minimale contributivo andasse ricavata da quelli stipulati dalle organizzazioni dei lavoratori e dei datori di lavoro comparativamente più rappresentative. Nei primi anni Duemila sembrava che il fenomeno si fosse «largamente ridimensionato» ${ }^{69}$. A seguito del deterioramento della situazione economica dovu-

non provare che a fare un esempio, di accordi conclusi su singoli istituti per "settori minori”).

66 Tra i primi a interrogarsi sul tema dei contratti "pirata”, D. GotTARDI, Significato e anomalia di un contratto, in Lav. Inf., 1997, p. 21 ss., A. LASSANDARI, Pluralità di contratti collettivi nazionali per la medesima categoria, cit., G. PerA, Note sui contratti collettivi «pirata», cit., p. 381 ss.

67 A. Lassandari, Pluralità di contratti collettivi nazionali per la medesima categoria, cit.

68 G. Pera, Note sui contratti collettivi «pirata», cit., p. 382.

69 M. Roccella, Contrattazione collettiva, azione sindacale, problemi di regolazione del mercato del lavoro, in Lav. Dir., 2000, p. 355. 
to alla crisi del $2008^{70}$, il fenomeno ha tuttavia assunto le dimensioni considerevoli viste supra. In tutti o quasi i settori contrattuali (con l'eccezione del pubblico, per ovvie ragioni legate all'operare del d.lgs. n. 165/2001) vi sono accordi con ambito di applicazione sovrapposto a quelli dei contratti collettivi stipulati dalle associazioni aderenti alle confederazioni storiche ${ }^{71}$. Sono stati messi in piedi, nel volgere di pochissimi anni, una serie di sistemi negoziali paralleli, da parte di coalizioni negoziali che sembrano dotate di una certa stabilità $^{72}$. In alcuni casi, le circostanze nelle quali si è pervenuti alla stipulazione dei contratti collettivi sono tali da far dubitare della genuinità dell'azione negoziale, se non della stessa alterità sostanziale tra le parti stipulanti ${ }^{73}$. In altri, pare che alla prova di un'indagine

70 Ma anche - osserva Lassandari - in corrispondenza di un processo di delegittimazione della contrattazione nazionale venuto negli stessi anni a maturazione: A. LASSANDARI, Sulla verifica di rappresentatività delle organizzazioni sindacali datoriali, in Dir Lav. Rel. Ind., 2017, pp. 4-6.

71 La questione è affrontata anche nell'ultimo rapporto che la Commissione Europea, nel contesto del Semestre europeo, ha dedicato al sistema-Paese Italia: v. il Country report 2019 - Italy, pp. 38-39.

72 Ne rappresenta un esempio il sistema contrattuale messo in piedi dalla $\mathrm{Fa}$ mar (Federazione autonoma dei movimenti associativi), che a sua volta afferma di aderire alla confederazione Confamar (Confederazione autonoma dei movimenti associativi di rappresentanza dei lavoratori e dei consumatori). In base all'archivio contratti Cnel, la Famar risulta firmataria di $45 \mathrm{Ccnl}$, così ripartiti tra i diversi settori contrattuali: 5 Agricoltura, 2 Chimici, 4 Metalmeccanici, 2 Tessili, 2 Alimentaristi-Agroindustriale, 3 Edilizia, 11 Commercio, 5 Trasporto, 8 Aziende di Servizi, 1 Enti e Istituzioni Private, 2 altri vari. Insomma, è ormai «riduttivo continuare a ritenere che solo Ugl, Cisal e Confsal arricchiscano il panorama delle confederazioni» (così, D. GOTTARDI, La contrattazione collettiva tra destrutturazione e ri-regolazione, cit., p. 887).

73 È il caso di dirlo, con riferimento ai Ccnl stipulati dalla confederazione Esaarco, che nel 2014 aveva stipulato, come capofila di coalizioni eterogenee, ben $38 \mathrm{Ccnl}$ nel medesimo giorno ( $1^{\circ}$ novembre 2014), con ambiti di applicazione che spaziavano sulla gran parte dell'arco dei settori dell'economia (lo aveva posto in luce G. Olini, I contratti nazionali: quanti sono e perché crescono, cit., p. 420). Che la capacità di mettere in piedi una tale singolare "economia di scala" sia un carattere distintivo dell'attività negoziale della confederazione datoriale Esaarco, è confermato dai rinnovi intervenuti nel 2018: anche questa volta in uno stesso giorno (il 30 marzo 2018) sono stati stipulati $38 \mathrm{Ccnl}$, in settori contrattuali che spaziano su tutto l'arco del tessuto produttivo; sono 6 nel settore agricoltura, 4 nel metalmeccanico, 2 in alimentaristi-agroindustriale, 4 in edilizia, 1 in poligrafici e spettacolo, 8 nel commercio, 2 nei trasporti, 2 in aziende di servizi, 7 in enti e istituzioni private, 1 sub altri vari. 
giudiziale più approfondita sia emersa l'inconsistenza dei sindacati dei lavoratori con i quali un'accreditata associazione datoriale aveva stipulato un contratto collettivo nazionale ${ }^{74}$.

I contratti collettivi "alternativi” prevedono, in molti casi, un trattamento economico inferiore e, quindi, più attraente, per chi fondi la competitività sul basso costo del lavoro ${ }^{75}$. Studi approfonditi hanno illustrato come le differenze non riguardino soltanto i minimi retributivi, bensì anche istituti del trattamento cd. normativo, come ad esempio la disciplina del lavoro straordinario o quella delle sospensioni del rapporto di lavoro per malattia o infortunio, ed altro ancora ${ }^{76}$. Talvolta, tali contratti collettivi intervengono sulla regolamentazione dei contratti di lavoro diversi da quello cd. standard, intercettando, cioè, il sistema di rinvii che la legge rivolge esclusivamente ai soggetti più accreditati del sistema di relazioni industriali, mediante la controversa

74 Si allude alla vicenda che nel 2014 ha portato al superamento della tradizionale unitarietà della contrattazione collettiva del settore delle agenzie assicurative in gestione libera: nel 2014 l'Associazione datoriale Sna ha rifiutato di rinnovare il Ccnl concluso, unitamente all'associazione datoriale Anapa (ex Unapass), con Fiba-Cisl, Fisac-Cgil e Uilca-Uil, per stipulare invece un Ccnl con i sindacati Fesica-Confsal e Confsal-Fisals (una breve ricostruzione in G. Piglialarmi, La rappresentatività tra libertà sindacale e categoria merceologica. A proposito di una recente riflessione di Pietro Ichino, in Boll. Adapt, 7 ottobre 2019, n. 35). Tale vicenda contrattuale ha avuto alcune ricadute giudiziali, specie per la pretesa dell'Inps di continuare a utilizzare come retribuzione base per il calcolo del minimale contributivo quella prevista dal Ccnl stipulato con le federazioni di categorie di Cgil, Cisl e Uil: un contenzioso dagli esiti non scontati, atteso che se, da una parte, si può ritenere (provarlo è comunque cosa diversa) che le federazioni aderenti a Cgil, Cisl e Uil siano più rappresentative dei sindacati Confsal, dall'altra parte le organizzazioni datoriali avevano, nelle rispettive categorie contrattuali, ciascuna una propria consistenza nel tessuto produttivo. In ogni modo, per l'affermazione di cui al testo («inconsistenza dei sindacati dei lavoratori») è significativo che il Trib. Genova, $1^{\circ}$ febbraio 2019, in Riv. Giur. Lav., 2019, II, p. 644, nota di D. GHigiarelli, pur facendo uso dei poteri istruttori ex art. 421 c.p.c., non sia riuscito a trovare «traccia dei sue sindacati Fesica-Confsal e Confsal-Fisals», atteso che all'indirizzo «dove ci sarebbe dovuto essere un rappresentante» è stato rinvenuto «un Club del Genoa». Un esito opposto in Trib. Pavia 26 febbraio 2019, sempre in Riv. Giur. Lav., 2019, II, p. 644, ma anche in Dir. Rel. Ind., 2019, p. 1215 ss., nota G. Piglialarmi.

75 M.G. Greco, Contrattazione collettiva, contratti pirata e regolamenti nella determinazione dei livelli retributivi nelle cooperative di produzione e lavoro, in Variaz. Temi Dir. Lav., 2017, p. 374.

76 Cfr. G. Rizzuto, P. TomassetTi, Il dumping contrattuale nel settore moda, Roma, Edizioni lavoro, 2019, p. 213 ss. 
formula della rappresentatività comparativamente maggiore ${ }^{77}$. Si è rimarcato inoltre come il dumping sul trattamento economico e/o normativo dei lavoratori rappresenti in alcuni casi soltanto

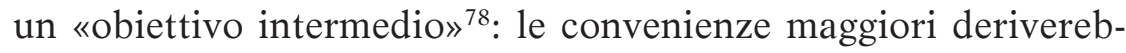
bero infatti dalla istituzione di patronati, enti bilaterali ${ }^{79}$, organismi per la formazione ${ }^{80}$ e così via.

La portata dirompente di questo fenomeno è chiara. Il modello italiano di relazioni industriali si identifica, dal dopoguerra in avanti, con il sistema contrattuale realizzato dai rapporti continuativi intercorsi tra le maggiori confederazioni dei lavoratori e dei datori di lavoro $^{81}$. Dal sistema sindacale di fatto, sul quale si fonda una delle intuizioni più feconde della cultura giuridica della materia ${ }^{82}$, dipende la tenuta dei livelli salariali, in assenza di una legge sul salario minimo ${ }^{83}$, e, più in generale, il rispetto di condizioni di lavoro adeguate.

Tuttavia, contrastare gli effetti distorsivi della proliferazione di contratti collettivi non è semplice, in un modello gius-sindacale

77 Istruttiva è ad esempio la vicenda della regolazione di un contratto parttime a zero ore, nel Ccnl intersettoriale - Commercio, terziario, distribuzione, servizi, pubblici esercizi e turismo, concluso da Cifa e Confsal (19 dicembre 2016): v. la nota critica di F. D’Addio, M. Tiraboschi, Part-time senza vincolo di orario minimo e sindacato comparativamente più rappresentativo, in Dir. Rel. Ind., 2017, p. 882 ss. $418,421$.

78 G. OlinI, I contratti nazionali: quanti sono e perché crescono, cit., pp.

79 M. Marazza, Perimetri e rappresentanze sindacali (dei datori di lavoro e dei lavoratori), cit., p. IX.

80 Come nel caso della vicenda decisa dal TAR Lazio 30 giugno 2015, n. 8765, in Dir. Rel. Ind., 2015, p. 1180, nota L. VARI.

81 È significativo che in alcune celebri elaborazioni dottrinali, la forza politico-sociale delle confederazioni sindacali storiche sia stata valorizzata al punto di affermare, a diritto costante, l'efficacia generale (o tendenzialmente tale) dei contratti collettivi stipulati unitariamente, per tutti i lavoratori della categoria produttiva o merceologica da essi individuata: cfr. P. IcHINo, Funzione ed efficacia del contratto collettivo nell'attuale sistema delle relazioni sindacali e nell'ordinamento statale, in Riv. Giur. Lav., 1975, I, p. 457 ss., G. Ferraro, Ordinamento, ruolo del sindacato, dinamica contrattuale di tutela, Padova, Cedam, 1981.

82 G. GiUgni, Introduzione allo studio dell'autonomia collettiva, Milano, Giuffrè, 1960.

83 M. Magnani, Il salario minimo legale, in Riv. It. Dir. Lav., 2010, I, pp. 776-779. 
in cui «tutti i passaggi logici del discorso sono controvertibili» ${ }^{84}$.

È scivolosa, oltre che ripida, se si può usare la metafora, la via che passa da un accertamento della natura autenticamente sindacale dell'azione negoziale svolta dai soggetti che stipulano i contratti collettivi "pirata". Un tale accertamento si baserebbe su un dato di effettività dell'autotutela, che sarebbe proprio dei "veri" sindacati soltanto $^{85}$. A tacer del fatto che non sarebbe facile fornire una nozione esatta di "pirata" e che gli esiti di un esame in concreto potrebbero non rivelarsi scontati, sorgerebbero comunque dubbi significativi. Da un lato, non sarebbe sempre facile distinguere una contrattazione collettiva genuinamente ablativa, frutto di compromesso sindacale, da una che abbia, invece, precisamente e soltanto lo scopo di offrire ai datori di lavoro uno strumento di riduzione dei costi della manodopera ${ }^{86}$. Dall'altro, e a parte i casi eclatanti, una verifica di questo tipo rischierebbe di risolversi in un apprezzamento tra mez-

84 Così, G. Pera, Note sui contratti collettivi «pirata», cit., p. 385, il quale, in questo passo rimasto celebre, aggiungeva che «di troppa o totale libertà, specie sul piano delle fonti, si può anche morire, cioè essere nell'incapacità di costruire in positivo».

85 Cfr. M.G. GRECo, Contrattazione collettiva, contratti pirata e regolamenti nella determinazione dei livelli retributivi nelle cooperative di produzione e lavoro, cit., pp. 374-377, che si rifà specialmente alla teorizzazione di Flammia.

${ }_{86} \mathrm{Al}$ riguardo, può essere istruttiva la vicenda del Ccnl per i dipendenti da Istituti e Imprese di vigilanza privata e servizi fiduciari (8 aprile 2013) stipulato tra Assiv-Confindustria, Legacoop Servizi, Federlavoro e servizi - Confcooperative, Agci-Servizi, con Filcams-Cgil e Fisascat-Cisl. Giova richiamare brevemente le circostanze che hanno portato alla stipulazione di questo contratto collettivo (sulle quali v. però ampiamente Trib. Torino 9 agosto 2019, n. 1128, in Lav. Dir. Eur., 2019, 3, nota L. IMBERTI, in Labor, 2020, p. 75 ss., nota M. GiACONI), che prevede livelli retributivi minimi particolarmente bassi per le posizioni di vigilantes meno qualificate. Sembra che Filcams-Cgil e Fisascat-Cisl si siano risolte a concludere questo contratto collettivo con retribuzioni sensibilmente inferiori a quelle previste, per mansioni analoghe, da altri Ccnl del circuito confederale, allo scopo di evitare che le aziende del settore si volgessero in blocco verso l'applicazione di un contratto collettivo stipulato da organizzazioni "meno rappresentative": si tratterebbe dunque di un caso emblematico di come il dumping effettuato tramite contratti collettivi "pirata" possa incidere negativamente sulle attività negoziali degli stessi soggetti più accreditati del sistema contrattuale. Tuttavia, è significativo che i livelli retributivi previsti da tale contratto collettivo stipulato dalle due federazioni di categoria probabilmente più rappresentative dell'intero comparto terziario (FilcamsCgil e Fisascat-Cisl) siano stati più volte, in giudizio, considerati contrari all'art. 36 Cost. (oltre a Trib. Torino n. 1128/2019, cit., v. Trib. Milano 30 giugno 2016, n. 1977, in Labor, 2017, p. 233 ss., nota G. Centamore, confermata da CA Milano 28 dicembre 2017, n. 1885, a quanto consta inedita). 
zi e fini dell'azione collettiva, ossia in un penetrante controllo giudiziale sull'operato delle organizzazioni sindacali, dalla non scontata compatibilità con l'art. 39 , c. 1 , Cost. ${ }^{87}$.

Peraltro, alcune tra le organizzazioni che, in questi anni, si associano al fenomeno della negoziazione "pirata", sono firmatarie di importanti accordi interconfederali sulla contrattazione collettiva ${ }^{88} \mathrm{e}$ sulla rappresentanza ${ }^{89}$; sono, cioè, soggetti accreditati dai principali attori del sistema di relazioni industriali, ai quali non sarebbe scontato potere attribuire l'etichetta di firmatari di accordi "non genuini". Tanto più che l'inapplicabilità ai rapporti di lavoro (men che meno l'invalidità $)^{90}$ di tali contratti collettivi non potrebbe essere affermata per essere la stipulazione effettuata da coalizioni minoritarie, solo che si consideri che lo stesso sistema sindacale di fatto si fonda su un principio di riconoscimento reciproco che non necessariamente si risolve nella realizzazione di una volontà maggioritaria ${ }^{91}$.

\subsubsection{Il dumping contrattuale interno al sistema di rappresentan- za "confederale"}

Sarebbe errato tuttavia ridurre l'intera questione ad una contrapposizione tra $«$ noi e loro $»^{92}$, tra sindacato tradizionale e agen-

87 Su questi temi, cfr. M. Persiani, Il contratto collettivo di diritto comune nel sistema delle fonti del diritto del lavoro, cit.

88 Oltre all'Accordo quadro del 22 gennaio 2009, v. (dopo il Protocollo d'intesa del 31 maggio 2013 con Cgil, Cisl e Uil), i Protocolli d'intesa stipulati tra Confindustria e Ugl il 6 giugno 2013, tra Confindustria e Cisal il 18 giugno 2013 e tra Confindustria e Confsal il 4 luglio 2013.

89 V. il Testo unico sulla rappresentanza stipulato, pochi giorni dopo rispetto alla stipulazione con Cgil, Cisl e Uil, tra Confindustria e Ugl il 10 gennaio 2014, tra Confindustria e Cisal il 14 gennaio 2014 e tra Confindustria e Confsal il 15 gennaio 2014; oppure il Testo unico sulla rappresentanza stipulato tra Confservizi e Cisal il 10 marzo 2014 e tra Confservizi e Ugl il 17 marzo 2014.

90 Cons. St. 13 ottobre 2015, n. 4699, in Riv. Giur. Lav., 2016, II, p. 20, nota D. CALDERARA.

91 M. PERSIANI, Le vicende della rappresentanza e rappresentatività sindacali tra legge e contratto collettivo, in Arg. Dir. Lav., 2017, p. 551.

92 Per riprendere il titolo di un saggio di U. Romagnoli («Noi e loro»: diritto del lavoro e nuove tecnologie, in Riv. Trim. Dir. Proc. Civ., 1986, p. 377 ss.), nel quale l'a. si confrontava con l'allora incipiente fenomeno dell'evoluzione tecnologica e con i suoi riflessi sul dibattito giuslavoristico. 
ti newcomers, tra sindacato rappresentativo e sindacato filo-padronale, tra prodotti contrattuali frutto di genuina azione industriale e contratti collettivi "pirata". In realtà, non mancano tensioni all'interno degli schieramenti dello stesso sistema confederale, anche (se non soprattutto) dal lato dei datori di lavoro, né ad esso sono estranee situazioni di vero e proprio dumping contrattuale, come emerge confrontando gli ambiti di applicazione dei contratti collettivi in alcune aree di particolare sofferenza del sistema negoziale ${ }^{93}$.

Tali svolgimenti si spiegano principalmente come una conseguenza dell'accresciuto livello di competizione tra le organizzazioni dei datori di lavoro ${ }^{94}$. Non sarebbe errato osservare che, mentre la triplice sindacale ha saputo ritrovare unità di azione, dopo le fratture della contrattazione separata e del caso Fiat ${ }^{95}$, le principali organizzazioni dei datori di lavoro hanno, invece, progressivamente distanziato gli uni dagli altri i propri sistemi contrattuali ${ }^{96}$, sia sul piano delle politiche salariali sia su quello (ancora in fieri) della misurazione della rappresentatività ${ }^{97}$.

La dottrina ha evidenziato le principali aree di tensione. Una prima si registra in relazione alle imprese di minori dimensioni, che, da una parte, costituiscono una delle storiche roccaforti di rappre-

93 M. Vitaletti, La rappresentatività sindacale "utile". Cosa resta del T.U. del 2014, in Dir. Lav. Merc., 2018, p. 42.

94 Vedi in questo senso il Documento unitario del 14 gennaio 2016 Cgil, Cisl, Uil per «Un moderno sistema di relazioni industriali», parte I «La contrattazione: riformare il modello per renderlo più inclusivo». In dottrina, cfr. L. BELLARDI, Il sistema di rappresentanza imprenditoriale e la struttura della contrattazione collettiva, cit., pp. 251-253, 260, M. MASCINI, Le relazioni industriali, in ID. (a cura di), l'Annuario del lavoro 2018, Roma, Il diario del lavoro, 2018, p. 36. Non sono mancati tuttavia i segnali di senso contrario, tra i quali la costituzione di umbrella associations nel settore terziario e delle cooperative: cfr. V. PAPA, Verso una rappresentanza misurata? Strumenti e metodi di misurazione della rappresentatività datoriale, cit., p. 23, nota 4.

95 F. Martelloni, Processo al sindacato, trent'anni dopo, in A. Lassandari, F. Martelloni (a cura di), Giorgio Ghezzi. Processo al sindacato, Roma, Ediesse, 2012, p. 147 ss.

96 V. PAPA, Verso una rappresentanza misurata? Strumenti e metodi di misurazione della rappresentatività datoriale, cit., p. 23.

97 In modo che appare persino «eclatante», ove si tenti un paragone «con quanto accaduto nell'ormai lontanissimo 1993»: così, A. LASSANDARI, Retribuzione e contrattazione collettiva, in Riv. Giur. Lav., 2019, I, p. 219. 
sentanza della Confindustria ${ }^{98}$, ma che, dall'altra, sono sempre più attratte dal dinamismo contrattuale delle organizzazioni degli artigiani e delle $\mathrm{PMI}^{99}$. Una seconda riguarda le imprese che forniscono servizi: un mercato in crescita, anche per i rapidi cambiamenti sociali e tecnologici che lo sospingono, e che è conteso sia dalle organizzazioni di area industriale sia da quelle del terziario ${ }^{100}$. Lo stesso comparto del terziario è interessato al suo interno da rilevanti fenomeni di competizione regolativa ${ }^{101}$.

Poiché le sfere di influenza non sono più ben definite nel sistema di relazioni sindacali, aumentano anche le situazioni di sovrapposizione tra i perimetri dei contratti collettivi di settore. Alcuni esempi possono dare l'idea della tendenza in atto.

Nel settore edile il Ccnl stipulato dalle principali associazioni di categoria, per aziende industriali e cooperative ${ }^{102}$, subisce una doppia concorrenza, se così la si può chiamare ${ }^{103}$ : da un lato, da parte del Ccnl Multiservizi, che include nella propria sfera di applicazione le attività di manutenzione di immobili e, dall'altro, da parte

98 G. Pera, Note sui contratti collettivi «pirata», cit., p. 384. V. altresì M. MASCINI, Le relazioni industriali, in ID. (a cura di), l'Annuario del lavoro 2017, Roma, Il diario del lavoro, 2017, pp. 40-41.

99 È significativo che, di recente, alcuni contratti collettivi di area artigiana abbiano apertamente esteso il proprio campo di applicazione alle piccole imprese tout court: si può ricordare il caso dei contratti delle imprese artigiane del settore alimentare e comunicazione, che con il rinnovo del 2010 «hanno esteso il proprio campo di applicazione alle aziende che, pur non rientrando nella fattispecie legale dell'impresa artigiana ex 1. n. 443/1985, presentano caratteristiche dimensionali simili. L'obiettivo esplicitamente perseguito è, infatti, proprio quello di favorire l'accesso delle piccole e medie aziende - finora inserite in altri sistemi di rappresentanza - ad un modello contrattuale più aderente alle esigenze di questa particolare dimensione produttiva»: così, L. BELLARDI, Il sistema di rappresentanza imprenditoriale e la struttura della contrattazione collettiva, cit., p. 260, la quale riprende sul punto M. Marazza, L'artigianato, in M. Mascini (a cura di), l'Annuario del lavoro 2010, Viterbo, Union Printing, 2010, p. 247 ss.

100 N. Penelope, La contrattazione interconfederale, in M. MAScINI (a cura di), l'Annuario del lavoro 2017, cit., p. 77.

101 M. Mascini, Le relazioni industriali, in M. MASCINI (a cura di), l'Annuario del lavoro 2017, cit., pp. 40-41.

102 Il Ccnl (ora 18 luglio 2018) è stipulato tra Ance, Legacoop produzione e servizi, Confcooperative lavoro e servizi, Agci produzione e lavoro, Feneal-Uil, Filca-Cisl e Fillea-Cgil.

103 V. Leccese, G. Roma, M. Lozito, La contrattazione nazionale di categoria, in M. Mascini (a cura di), l'Annuario del lavoro 2018, cit., p. 80. 
del Ccnl meccanici artigiani, che, in virtù di un'estensione dell'area contrattuale decisa dalle parti con il rinnovo del 2018, annovera nel campo di applicazione le attività di montaggio e smontaggio di ponteggi edili ${ }^{104}$. Un secondo esempio, che di recente ha sollecitato l'interesse della dottrina lavoristica ${ }^{105}$ e sociologica ${ }^{106}$, riguarda il Ccnl Alimentari industria, la cui applicazione è insidiata dai Ccnl della Logistica ${ }^{107}$ e Multiservizi, che prevedono un trattamento economico e normativo minore rispetto al primo ed annoverano posizioni professionali "adattabili" alle attività di lavorazione dei prodotti alimentari. Una terza situazione, giunta all'attenzione anche della giurisprudenza, concerne le attività di vigilanza non armata, custodia, portierato $\mathrm{e}$ simili ${ }^{108}$. Tali attività sono regolate da almeno quattro contratti collettivi, stipulati da associazioni di categoria certamente tra le più rappresentative, a livello nazionale, nei propri ambiti di azione: il Ccnl del Commercio, il Ccnl Multiservizi, il Ccnl per i dipendenti di proprietari

104 Ccnl Meccanica, orafi, argentieri e affini, odontotecnica, art. 1 - Sfera di applicazione; il contratto collettivo (rinnovo 24 agosto 2018) è stipulato tra CNA Produzione, CNA Installazione di impianti, CNA Servizi alla comunità/Autoriparatori, CNA artistico e tradizionale, CNA Benessere e sanità, Confartigianato Autoriparazione, Confartigianato Metalmeccanica di produzione, Confartigianato impianti, Confartigianato Orafi, Confartigianato odontotecnici, Casartigiani, Claai, FiomCgil, Fim-Cisl e Uilm-Uil.

105 S. BATtistelli et al., Structural characteristics and industrial relations in the pork value chain: the case of Italy, in P. CAmpanella, D. Dazzi (eds.), Meatup Ffire Fairness freedom and industrial relations across Europe: up and down the meat value chain, Milano, Franco Angeli, 2020, p. 139 ss., O. BonARDI, Tra governance della global value chain e frammentazione del tessuto produttivo nazionale: quale regolamentazione per il lavoro nel settore della logistica?, in A. Allamprese, O. BONARDI (a cura di), Logistica e lavoro, cit., p. 34.

106 L. DorigatTi, A. Mori, L'impatto delle scelte datoriali sulle condizioni di lavoro e sulle diseguaglianze: disintegrazione verticale, esternalizzazioni e appalti, in Soc. Lav., 2016, p. 190 ss.

107 Ccnl logistica, trasporto, merci e spedizione (3 dicembre 2017) tra Aite, Aiti, Assoespressi, Assologistica, Fedespedi, Fedit, Fisi, Trasportounito Fiap, assistite dalla Confetra, Anita, Fai, Assotir, Federtraslochi, Federlogistica, Fiap, Unitai, assistite dalla Conftrasporto, Cna Fita, Confartigianato Trasporti, Sna Casartigiani, Claai, Filt-Cgil, Fit-Cisl e Uiltrasporti-Uil.

108 Tra le più recenti vedi Cass. 20 febbraio 2019, n. 4951, in Labor, 2019, p. 401 ss., nota L. ImberTi, Cass. 21 febbraio 2019, n. 5189, Cass. $1^{\circ}$ marzo 2019, n. 6143, entrambe in Pluris. In giurisprudenza di merito, Trib. Torino n. 1128/2019, cit. 
di fabbricati ${ }^{109}$ e il Ccnl Vigilanza non armata - sezione servizi fiduciari, che prevedono per una medesima attività di lavoro trattamenti economici sensibilmente diversi. Simili questioni ha affrontato la giurisprudenza ${ }^{110}$, in relazione ai Ccnl Cooperative sociali ${ }^{111}$, Igiene urbana $^{112}$ e Multiservizi, che sono tutti in qualche misura riconducibili, come campo di applicazione, alle attività di igiene urbana.

4. Il percorso a ostacoli per una riorganizzazione del sistema contrattuale

I descritti svolgimenti interni al sistema della contrattazione collettiva ne hanno messo apertamente in discussione uno dei pilastri ${ }^{113}$, ossia la tendenziale unicità dei contratti collettivi nei loro ambiti di applicazione. Ciò complica non poco l'attività di selezione dei contratti collettivi da utilizzare quale parametro del trattamento, nei casi di rinvio all'autonomia collettiva (v. infra, capitolo IV), ma, per la verità, a (rischiare di) divenire ingovernabile è ancor prima lo stesso sistema contrattuale.

Non stupisce quindi che l'esigenza di una semplificazione del sistema contrattuale sia da anni nell'agenda delle parti sociali, che sembrano porsi una serie di obiettivi. Un primo obiettivo consiste nel ridurre il numero di Ccnl "vigenti", con operazioni di accorpamento tra unità negoziali affini, oppure facendo confluire le aree di Ccnl poco applicati verso Ccnl più "attivi", solo per fare alcuni

109 Ccnl per i dipendenti di proprietari di fabbricati (12 novembre 2012), stipulato tra Confedilizia, Filcams-Cgil, Fisascat-Cisl, Uiltucs-Uil.

110 Cass. 27 marzo 2019, n. 8592.

111 Ccnl Servizi socioassistenziali cooperative (16 dicembre 2011) tra Agci Solidarietà, Legacoop Sociali, Federsolidarietà Confcooperative, FP-Cgil, FP-Cisl, Fisascat-Cisl e FPL-Uil.

112 Ccnl Pulizia Igiene urbana Private, 20 giugno 2017, stipulato tra Fise Assoambiente, Fise, FP-Cgil, Fit-Cisl, Uiltrasporti e Fiadel (sottoscrizione per adesione da parte di Ugl e Ugl Igiene ambientale 12 luglio 2017).

113 S. Scarponi, Rappresentatività e organizzazione sindacale, Padova, Cedam, 2005, pp. 122-123. 
esempi ${ }^{114}$. Un secondo consiste nel rivedere, sia pure gradualmente, le clausole sugli ambiti di applicazione dei contratti collettivi, in modo da «garantire una più stretta correlazione tra CCNL applicato e reale attività d'impresa» ${ }^{15} \mathrm{e}$, per questa via, ridurre di numero e di intensità i conflitti di giurisdizione interni al sistema confederale ${ }^{116}$. Il terzo obiettivo è il contrasto alla contrattazione collettiva "pirata".

Il punto di partenza del processo di riforma del sistema contrattuale può essere individuato nel documento unitario di Cgil, Cisl e Uil del maggio 2008, recante «Linee di riforma della struttura della contrattazione». Si è già avvertito che le parti stipulanti manifestavano già allora l'esigenza di «una verifica in ordine alla razionalizzazione delle aree di copertura dei CCNL (oltre 400) prevedendo la possibilità di accorpamenti per aree omogenee e per settori, favorendo la riunificazione di contratti analoghi facenti riferimento a diverse organizzazioni di rappresentanza datoriale».

Nel 2009 è stato poi stipulato, tra governo e parti sociali (con

114 Due esempi possono essere tratti dal comparto artigianato - piccole e medie imprese: i) il Ccnl per i dipendenti della piccola e media industria moda - chimica ceramica - decorazione piastrelle in terzo fuoco del 7 novembre 2017, concluso tra Cna Federmoda, Cna produzione, Cna artistico e tradizionale, Confartigianato moda, Confartigianato chimica, Confartigianato ceramica, Casartigiani, Claai, con Filctem-Cgil, Femca-Cisl, Uiltec-Uil, ha rinnovato e accorpato i Ccnl scaduti di area tessile-moda e chimica-ceramica; $i$ ) il Ccnl area meccanica per i lavoratori dipendenti dalle imprese artigiane dei settori metalmeccanica, installazione di impianti, orafi, argentieri ed affini, e dalle imprese odontotecniche del 16 giugno 2011 ha accorpato i tre Ccnl (tutti scaduti nel 2008) del settore artigiano metalmeccanica ed installazione di impianti, del settore artigiano orafi, argentieri ed affini e del settore odontotecnica (vedi la premessa al Ccnl). Altri esempi in V. Leccese, G. Roma, M. Lozito, La contrattazione nazionale di categoria, in M. MascinI (a cura di), l'Annuario del lavoro 2019, cit., pp. 68, 71-72 (per il settore dei materiali da costruzione), F. Siotтo, La categoria come ambito di applicazione e perimetro di misurazione della rappresentanza, cit., p. 317 nota 19 (per il nuovo Ccnl unico dell'industria armatoriale) e in V. PAPA, Verso una rappresentanza misurata? Strumenti e metodi di misurazione della rappresentatività datoriale, cit. p. 25 nota 9 (per il settore delle public utilities).

115 Si trae dal cd. Patto per la fabbrica del marzo 2018.

116 In alternativa ad una vera e propria revisione delle clausole sul campo di applicazione dei contratti collettivi, sarebbe anche pensabile (e forse meno impegnativa) l'adozione di note di chiarimento e di esplicazione da parte delle organizzazioni stipulanti, con le quali si indichi in modo non equivocabile se certe attività rientrano o meno nelle aree contrattuali. 
l'eccezione significativa della Cgil) ${ }^{117}$, l'accordo quadro di «riforma degli assetti contrattuali» ${ }^{118}$, al cui punto 19 si prevede che «le parti convengono sull'obiettivo di semplificare e ridurre il numero dei contratti collettivi nazionali di lavoro nei diversi comparti». All'accordo quadro seguivano accordi interconfederali conclusi, senza l'adesione della Cgil, per le aree confindustriale (15 aprile), dell'artigianato (23 luglio) e di Confservizi (18 novembre), oltre a un protocollo d'intesa (unitario) per l'agricoltura (22 settembre). Ciascuno di tali accordi ribadiva la volontà delle parti sociali di semplificare il quadro dei contratti collettivi di categoria' ${ }^{119}$. Per l'area dell'artigianato veniva concluso (28 settembre) uno specifico accordo «per la semplificazione dei contratti collettivi nazionali di lavoro nell'artigianato e sui settori scoperti»: tale accordo stabiliva le disposizioni da osservare, in sede di rinnovi, al fine di procedere con gli accorpamenti dei Ccnl in un numero più limitato, rispetto al passato, di macroaree contrattuali ${ }^{120}$.

117 L'accordo è stipulato tra il Governo e Cisl, Uil, Ugl, Cisal, Confsal, Sin.Pa, Confindustria, Confcommercio, Confesercenti, Confapi, Confservizi, Confetra, Confartigianato, Cna, Casartigiani, Claai, Confagricoltura, Coldiretti, Cia, Copagri, Lega delle cooperative, Confcooperative, Unci, Agci, Unione italiana cooperative, Cida, Confedir, Ciu Unionquadri, Confail, Cuq, Assolavoro, Confedertecnica e Confprofessioni.

118 Cfr. F. CARINCI, Una dichiarazione d'intenti: l'Accordo Quadro 22 gennaio 2009 sulla riforma degli assetti contrattuali, cit., L. BELLARDI, L'attuazione dell'accordo quadro: pluralità dei sistemi contrattuali ed eterogenesi dei fini. Alcune note di sintesi, cit., A. LASSANDARI, La contrattazione collettiva: prove di de-costruzione di un sistema, in Lav. Dir., 2011, p. 321 ss.

119 L'AI Confindustria, Cisl e Uil del 15 aprile 2009, al punto 8 «Razionalizzazione e riduzione del numero dei contratti collettivi nazionali di lavoro di categoria» manifesta tale volontà, ma precisa che dovrà su ciascuna ipotesi «essere verificato lo specifico interesse reso esplicito dalle Associazioni/Federazioni di Categoria titolari dei rispettivi contratti nazionali». (Anche) a tale scopo veniva istituita una sede bilaterale (Comitato paritetico, spec. dall'allegato all'AI). Formulazione e rubrica identiche si ritrovano nell'AI Confservizi, Cisl e Uil del 18 novembre 2009 (punto 7). L'AI 23 luglio 2009 tra Confartigianato imprese, Cna, Casartigiani, Claai, con Cisl e Uil (attuativo dell'intesa del 21 novembre 2008) al n. 6 «Nuove aree contrattuali» stabilisce, in applicazione dell'intesa del 2008, che una commissione tecnica bilaterale «si insedierà per definire gli ambiti di applicazione dei 9 CCNL d'area e per dare copertura contrattuale ai settori scoperti». Il protocollo d'intesa del 22 settembre 2009 tra Confagricoltura, Coldiretti, Cia, Flai-Cgil, Fai-Cisl e Uila-Uil, al n. 9 «Razionalizzazione e armonizzazione dei contratti collettivi», allude a un interesse delle parti stipulanti «a proseguire nell'attività di verifica circa la possibilità di semplificazione, di razionalizzazione o di armonizzazione dei contratti collettivi».

120 AI 28 settembre 2009 (medesime parti firmatarie dell'AI del luglio), con il quale si stabilivano, n. 1): sulla base di 9 macro-aree contrattuali, una serie di 
Con alcune significative eccezioni (tra le quali l'area artigiana), le previsioni del 2009 sono rimaste, tuttavia, in larga misura, prive di seguito, per quanto attiene alla questione di una risoluta semplificazione dei contratti di categoria. Un obiettivo di questa portata non avrebbe potuto, del resto, essere conseguito senza un elevato livello di condivisione tra le strutture confederali, da una parte, ed un appoggio da parte delle federazioni di categoria, dall'altra $^{121}$. Le intese interconfederali erano non soltanto prive del sostegno, non secondario, della Cgil, ma anche impotenti, di fronte alle questioni più spinose ${ }^{122}$, di definizione dei confini tra le aree di rappresentanza delle principali confederazioni datoriali. Né sembra che il coinvolgimento delle federazioni di categoria - che sono i soggetti «titolari dei contratti collettivi» ${ }^{123}$ - sia andato oltre mere «dichiarazioni di intenti» ${ }^{124}$.

Il tema sarebbe, peraltro, svanito, per alcuni anni, dall'agenda

accorpamenti tra contratti collettivi di categoria, da realizzarsi nella successiva tornata di rinnovi; n. 2): i criteri da rispettare per procedere con tali accorpamenti e tenere al contempo conto delle specificità dei settori che sarebbero confluiti in Ccnl unitari; n. 3): disposizioni volte ad estendere la copertura contrattuale a settori in precedenza «scoperti». I successivi rinnovi avrebbero dato seguito a tali previsioni. Il più recente $\mathrm{AI}$ del 23 novembre 2016 (Confartigianato imprese, Cna, Casartigiani, Claai, con Cgil, Cisl e Uil) sulle «linee guida per la riforma degli assetti contrattuali e delle relazioni sindacali», al n. 1 («Gli assetti contrattuali»), lett. $g$ ) («razionalizzazione CCNL») prevede di proseguire nel percorso avviato dieci anni prima: «Le parti convengono di procedere ad ulteriori accorpamenti per pervenire alle seguenti quattro macro-aree» (manifatturiero, servizi, edilizia, autotrasporto).

121 Diversamente, emergerebbe il rilievo di indicazione soltanto politico-sindacale degli accordi interconfederali: cfr. P. Tosi, Gli assetti contrattuali fra tradizione e innovazione, in Arg. Dir. Lav., 2013, pp. 518, 548 ma passim. Anni prima, T. Treu, Il conflitto e le regole, cit., p. 320, osservava che «anche le proposte più stringenti di regolazione provenienti dai vertici confederali» corrono il rischio di «vanificarsi nella giungla della settorializzazione [...] contrattuale».

122 A. LasSANDARI, Sulla verifica di rappresentatività delle organizzazioni sindacali datoriali, cit., pp. 15-16.

123 L'espressione tra virgolette è forse poco felice, ma, oltre a rendere l'idea del concetto che si vuole esprimere, rientra nel lessico delle relazioni industriali: essa è utilizzata dall'AI Confindustria, Cisl e Uil del 15 aprile 2009, punto 8, cit., così come negli AI successivi che hanno dedicato disposizioni al tema della «Titolarità ed efficacia della contrattazione collettiva».

124 Per riprendere F. CARINCI, Una dichiarazione d'intenti: l'Accordo Quadro 22 gennaio 2009 sulla riforma degli assetti contrattuali, cit., pp. 194-195. 
delle parti sociali, in corrispondenza delle vicende di contrattazione separata del Ccnl metalmeccanici industria (2009), del Ccnl terziario, distribuzione e servizi (2011) e della contrapposizione sindacale e giudiziaria tra gruppo FCA e Fiom-Cgil. I nodi da sciogliere per le parti sociali riguardavano, in quella fase, soprattutto il decentramento della struttura contrattuale, la contrattazione in deroga e le strutture di rappresentanza dei lavoratori in azienda.

Il problema di una razionalizzazione dei perimetri contrattuali è riproposto, tuttavia, dagli accordi interconfederali unitari conclusi, dal 2011, a partire dall'area-guida confindustriale ${ }^{125}$, ma via via per tutti i principali comparti dell'economia. Tale problema rappresenta, invero, uno dei convitati di pietra dei meccanismi di verifica della rappresentatività sindacale, previsti per l'accesso alle trattative e per la stipulazione maggioritaria dei contratti collettivi ${ }^{126}$.

Logica vuole che, per procedere in questa direzione, si stabiliscano per prima cosa le unità per le quali effettuare la misurazione della rappresentatività. Si è osservato a questo proposito che «L'ambito di misurazione della rappresentatività ha carattere costitutivo della stessa» ${ }^{127}$. Altri ha precisato che delimitare a monte il gruppo d'interesse nell'ambito del quale effettuare un'operazione deliberativa è uno dei problemi centrali della democrazia sindacale ${ }^{128}$.

Le formulazioni dei primi accordi del 2011-2012 facevano riferimento ad una rappresentatività calcolata su un generico complesso di «lavoratori della categoria cui si applica il contratto col-

125 M. BARBIERI, Note critiche sul testo unico sulla rappresentanza sindacale, in L. Zoppoli, A. Zoppoli, M. Delfino (a cura di), Una nuova Costituzione per il sistema di relazioni sindacali?, cit., p. 211.

126 A. Viscomi, Prime note sul protocollo 31 maggio 2013, in Riv. It. Dir. Lav., 2013, I, p. 764, per il quale nell'accordo «l'ambito di applicazione [dei contratti collettivi] è invocato a monte per la definizione della rappresentatività». Dello stesso avviso è M. MAGNANI, Rappresentatività e diritti sindacali tra autonomia collettiva e giustizia costituzionale, in F. CARINCI (a cura di), Legge o contrattazione? Una risposta sulla rappresentanza sindacale a Corte costituzionale n. 231/2013, Adapt Labour Studies, E-Book Series, n. 20, 2014, p. 61.

127 A. Zoppoli, Rappresentanze sindacali e rappresentatività, le insidie del tempo, in Dir. Lav. Merc., 2018, p. 366.

$128 \mathrm{~V}$. BAVARO, Il principio maggioritario nelle relazioni industriali, in Lav. Dir., 2014, p. 19. 
lettivo nazionale di lavoro» ${ }^{129}$, o di organizzazioni «che abbiano nel settore una rappresentatività.... ${ }^{130}$. Si trattava di formulazione generiche, inadatte ad individuare in modo adeguato il bacino di misurazione della rappresentatività delle organizzazioni firmatarie dei contratti collettivi. Gli accordi degli anni seguenti avrebbero adottato formulazioni più precise. In particolare, dal Protocollo d'intesa del 31 maggio 2013 in avanti, si sarebbe stabilito di misurare la rappresentatività sulla base degli ambiti di applicazione dei contratti collettivi ${ }^{131}$, ossia, per così dire, dell'unico "insieme" che l'art. 39, c. 1, Cost. consente alle parti sociali di definire esattamente.

Ciò si ricava dagli accordi conclusi in area Confindustria ${ }^{132}$,

129 (Corsivo mio). Si cita dall'AI 28 giugno 2011 tra Confindustria e Cgil, Cisl e Uil, punto 1 , in materia di legittimazione a negoziare i contratti collettivi nazionali (era indicata, in particolare, una soglia del 5\% di rappresentatività, calcolata come media tra dato associativo ed elettorale).

130 (Corsivo mio). Così, sulla scia dell'AI 28 giugno 2011, ma con la variazione semantica indicata nel testo, sia l'AI tra Confservizi e Cgil, Cisl e Uil del 21 dicembre 2011, punto 1, sia l'AI «in materia di rappresentanza» del 20 aprile 2012 tra Confapi e Cgil, Cisl e Uil, punto 1, sempre in materia di ammissione alle trattative per la contrattazione collettiva nazionale (soglia del 5\% di rappresentatività, calcolata come media tra dato associato ed elettorale).

131 A. Maresca, Il contratto collettivo nazionale di categoria dopo il Protocollo d'intesa 31 maggio 2013, in Riv. It. Dir. Lav., 2013, I, pp. 719-721.

132 Il Protocollo d'intesa del 31 maggio 2013 tra Confindustria e Cgil, Cisl e Uil, sub «misurazione della rappresentatività», n. 2, prevede che tale misurazione debba avvenire, per quanto attiene alla raccolta delle deleghe sindacali, «per ambito di applicazione del contratto collettivo nazionale di lavoro di competenza»e, per il dato elettorale, «per ambito contrattuale». Le disposizioni del Protocollo sono sviluppate e precisate dal Testo unico sulla rappresentanza del 10 gennaio 2014, concluso tra le stesse parti. La parte I («misura e certificazione della rappresentanza ai fini della contrattazione collettiva nazionale di categoria») prevede che il dato di rappresentanza sarà «relativo ai singoli contratti collettivi nazionali di lavoro». La parte III ( «Titolarità ed efficacia della contrattazione collettiva nazionale di categoria ed aziendale») stabilisce che il diritto a partecipare alle trattative per la stipulazione dei contratti collettivi sia attribuito alle organizzazioni che abbiano una rappresentatività non inferiore al 5\% «nell'ambito di applicazione del contratto collettivo nazionale di lavoro». Conferma questa linea anche l'accordo di modifica al Testo unico concluso tra le medesime parti il 4 luglio 2017, con il quale le parti manifestavano l'intenzione di trasferire all'Inps le prerogative in precedenza attribuite al Cnel. Vedi a conferma anche le convenzioni stipulate tra le parti sociali e l'Inps, una prima il 16 marzo 2015 e una seconda tra le stesse parti, con l'aggiunta dell'Inl, il 19 settembre 2019. 
Confservizi ${ }^{133}$, Confapi ${ }^{134}$, Confcommercio ${ }^{135}$, Confesercenti ${ }^{136}$, del-

133 V. il Protocollo d'intesa concluso il $1^{\circ}$ agosto 2013 tra Confservizi e Cgil, Cisl e Uil, che, sub «Misurazione della rappresentatività», prevede, al punto 2), che il dato associativo sia raccolto «per ambito di applicazione del contratto collettivo nazionale di lavoro» e al punto 4) che il dato elettorale sia raccolto «per ambito contrattuale» e che la ponderazione tra i due sarà effettuata «per ogni contratto collettivo nazionale di lavoro»; sub «Titolarità ed efficacia della contrattazione», il Protocollo prevede che saranno ammesse alla contrattazione le Federazioni di categoria che abbiano una rappresentatività non inferiore al 5\% «nell'ambito di applicazione del contratto collettivo nazionale di lavoro». Analogamente, le disposizioni del Testo unico sulla rappresentanza del 10 febbraio 2014 concluso tra le stesse parti. V. anche la convenzione stipulata tra le parti sociali e l'Inps in data 16 marzo 2016, che ha affidato all'Inps le attività di raccolta, elaborazione e comunicazione del dato associativo.

134 V. l'AI in materia di rappresentanza del 26 luglio 2016 tra Confapi e Cgil, Cisl e Uil: nella Parte I «Misura e certificazione della rappresentanza ai fini della contrattazione collettiva nazionale di categoria», si prevede che ai fini della misurazione della rappresentatività delle organizzazioni sindacali sia il dato associativo sia il dato elettorale siano rilevati e ponderati per «ambito di applicazione del CCNL»; nella Parte III «Titolarità ed efficacia della contrattazione collettiva nazionale di categoria, aziendale e territoriale», si attribuisce la «titolarità» della contrattazione collettiva alle federazioni di categoria che abbiano una rappresentatività non inferiore al 5\% «nell'ambito di applicazione del contratto collettivo». Conferma questa linea l'accordo di modifica del 23 settembre 2019, con il quale le parti manifestano l'intenzione di attribuire all'Inps le prerogative prima attribuite al Cnel. V. anche la convenzione stipulata tra le parti sociali, l'Inps e l'Inl il 27 settembre 2019.

135 V. l'AI del 26 novembre 2015 tra Confcommercio - Imprese per l'Italia e Cgil, Cisl e Uil, lett. $A$ ) «contrattazione collettiva nazionale di categoria», punto 3 ) «Modalità di rilevazione della rappresentatività»: I) le parti prevedono che il dato associativo sia rilevato «per ambito di applicazione del contratto collettivo nazionale di lavoro»; sub II-III) che il dato elettorale sia rilevato «per ambito di applicazione del contratto collettivo nazionale di lavoro di competenza»; sub IV-V) che la ponderazione tra dato associativo ed elettorale avverrà «per ogni contratto collettivo nazionale di lavoro». Al punto 4) «Titolarità ed efficacia della contrattazione», $s u b$ I) si stabilisce che parteciperanno alla contrattazione collettiva nazionale le organizzazioni di categoria con una rappresentatività non inferiore al 5\% «nell'ambito di applicazione del contratto collettivo nazionale di lavoro». L'AI del 24 novembre 2016 concluso tra le medesime parti conferma tali regole.

136 Disposizioni di tenore analogo a quelle stabilite in area Confcommercio - Imprese per l'Italia sono contenute nell'AI sul modello di relazioni sindacali del 7 settembre 2017 tra Confesercenti e Cgil, Cisl e Uil; in particolare, v. lett. A) «contrattazione collettiva nazionale di categoria», punto 3) «Modalità di rilevazione della rappresentatività» e punto 4) «Titolarità ed efficacia della contrattazione». 
la cooperazione ${ }^{137}$ e delle organizzazioni dell'artigianato e delle $\mathrm{PMI}^{138}$.

Tali accordi hanno stabilito che, una volta implementati, i meccanismi di misurazione della rappresentatività dei sindacati dei lavoratori opereranno sulla base delle unità definite mediante gli ambiti di applicazione dei contratti collettivi di categoria.

Le critiche avanzate da una parte della dottrina a questo tipo di sistema sono sostanzialmente due. La prima consiste nel rimarcare che, in questo modo, si corre il rischio di ingessare le dinamiche negoziali, dato che «la previsione contrattuale presuppone che le categorie contrattuali, e le relative parti stipulanti, siano quelle ad oggi esistenti, e non contempla l'eventualità di un loro mutamento» ${ }^{139}$ : non sarebbe, dunque, ammissibile una tale «sclerotizzazione delle

137 L’AI del 18 settembre 2013 concluso tra Agci, Confcooperative, Legacoop e Cgil, Cisl e Uil, lett. $B$ ) «con riferimento alla contrattazione collettiva nazionale di categoria», punto 2) «misurazione della rappresentatività», sub IIIV) prevede che il dato associativo sarà raccolto «per ambito di applicazione del contratto collettivo nazionale di lavoro di competenza», che il dato elettorale sarà raccolto «per ambito contrattuale» e che la ponderazione sarà effettuata «per ogni contratto collettivo nazionale di lavoro». Al punto 3) «Titolarità ed efficacia della contrattazione», si prevede che la contrattazione collettiva nazionale sia svolta dalle Federazioni di categoria che dispongano di una rappresentatività non inferiore al 5\% «nell'ambito di applicazione del contratto collettivo nazionale di lavoro». L’AI concluso tra le stesse parti il 28 luglio 2015 contiene disposizioni analoghe, in merito all'ambito nel quale misurare la rappresentatività delle organizzazioni sindacali, anche ai fini della titolarità della contrattazione collettiva. L'accordo interconfederale «sulle linee guida per la riforma delle relazioni industriali» concluso tra le stesse parti il 12 dicembre 2018 non prevede novità a questo proposito.

138 L'AI sulla rappresentanza del 23 novembre 2016 tra Confartigianato imprese, CNA, Casartigiani, Claai e Cgil, Cisl e Uil, «Misura e certificazione della rappresentatività e della rappresentanza ai fini della contrattazione collettiva nazionale di categoria», lett. $A$ ) «criteri di misurazione della rappresentatività e rappresentanza», punto 1) «certificazione e misurazione dei dati associativi», prevede che le deleghe sindacali saranno raccolte in relazione a «ciascun ambito di applicazione del CCNL». Alla lett. $C$ ) «misurazione e certificazione della rappresentanza», le parti prevedono che la ponderazione sarà effettuata in relazione «ai singoli contratti collettivi nazionali di lavoro e alle singole realtà territoriali».

139 Tra gli autori che hanno sostenuto questa posizione cfr. specialmente A. TURSI, L'accordo del 31 maggio su rappresentanza e rappresentatività per la stipula dei Ccnl: appunti in tema di rappresentatività, legittimazione negoziale, efficacia soggettiva e contrasto agli “accordi separati”, in Dir. Rel. Ind., 2013, p. 643. 
categorie contrattuali» ${ }^{140}$. Per una seconda opinione critica, un meccanismo di questo tipo non potrebbe comunque impedire alle federazioni di categoria o alle organizzazioni extra-confederali di modificare le categorie preesistenti, sulle quali sia stata operata la misurazione, o di individuarne di nuove, finendo, in ogni caso, per vanificare il corretto andamento delle verifiche sulla rappresentatività ${ }^{141}$. $\mathrm{Al}$ proposito, si è poi sostenuto che il fatto stesso di aver adottato un meccanismo di misurazione della rappresentatività basato sugli ambiti di applicazione dei contratti collettivi abbia agito, dal 2014 in avanti, per una singolare eterogenesi dei fini, quale «moltiplicatore del dumping contrattuale» ${ }^{142}$.

Queste critiche, tuttavia, non sembrano cogliere pienamente nel segno.

Rispetto alla prima, occorre riconoscere che qualsiasi meccanismo di verifica della rappresentatività sindacale non può funzionare, a meno che non sia prima «ben individuato e delimitato l'ambito nel quale la misurazione deve avvenire» ${ }^{143}$. Le parti sociali sono i soggetti cui una tale valutazione deve essere rimessa ${ }^{144}$ ed esse hanno

140 V. PINTO, Gli accordi interconfederali del 2013 e i persistenti problemi teorici (e pratici) della definizione autonoma della "categoria contrattuale", cit., p. 368; si è già avvertito che tale autore risolveva la questione in via interpretativa, sostenendo che la competenza a modificare le categorie contrattuali, per effetto degli accordi del 2013, fosse transitata implicitamente dalle federazioni di categoria verso le confederazioni: si è già illustrata, tuttavia, la ragione per la quale tale opinione non può essere accolta (v., supra, in questo capitolo, sub § 1).

141 M. MarazZA, Dalla "autoregolamentazione" alla "legge sindacale”? La questione dell'ambito di misurazione della rappresentatività sindacale, cit., passim, il quale, anche per tale ragione, si interroga sull'eventualità di sopperire alle carenze dell'accordo per via legislativa; sulla stessa linea mi sembra porsi M. VITALETTI, La rappresentatività sindacale “utile”. Cosa resta del T.U. del 2014, cit., p. 42.

142 Se ben si coglie, è questa la posizione di M. Marazza, Perimetri e rappresentanze sindacali (dei datori di lavoro e dei lavoratori), cit., p. VIII, ma passim, secondo il quale le parti sociali (area confindustriale) vi avrebbero posto rimedio mediante il Patto per la fabbrica del 2018.

143 Così, R. Del Punta, Note sparse sul Testo Unico sulla rappresentanza, in Dir. Rel. Ind., 2014, p. 676.

144 A. LASSANDARI, Sulla verifica di rappresentatività delle organizzazioni sindacali datoriali, cit., pp. 15-16 (l'autore si interrogava sull'eventualità che a stabilire un meccanismo del genere fosse una legge, ma le sue considerazioni valgono a maggior ragione ove la prospettiva resti quella interna alle relazioni industriali). 
esercitato tale prerogativa con una serie di accordi interconfederali. A ciò non si può non attribuire un significato, anche alla luce del principio di libertà sindacale di cui all'art. 39, c. 1 , Cost., che certamente consente alle parti sociali di rivedere nel tempo le proprie determinazioni in ordine al governo della struttura contrattuale. Peraltro, è più che ragionevole che tali unità siano state individuate nei perimetri dei contratti collettivi «sinora stipulati e vigenti, rispetto ai quali è dunque possibile avviare il sistema di misurazione con poche incertezze» ${ }^{145}$. È una logica lineare, di effettività delle relazioni industriali, che non impedirebbe, di per sé, in futuro di rivedere i confini della contrattazione collettiva (e, così, della misurazione di rappresentatività), dato che il rilievo è sempre e soltanto rebus sic stantibus $^{146}$.

In altri termini, se non è ovviamente immaginabile una cristallizzazione definitiva dei perimetri della contrattazione collettiva, lo è una loro momentanea stabilizzazione, specie se intervenuta per volontà delle stesse parti sociali. Al proposito, è significativo che siano stati indirettamente stabiliti congegni in grado di garantire l'evoluzione del sistema contrattuale. In particolare, si trae dalla convenzione stipulata il 19 settembre 2019 tra Inps, Inl, Confindustria, Cgil, Cisl e Uil, per rendere operativi i meccanismi di verifica della rappresentatività, l'impegno delle parti sociali (art. 2) «a comunicare tempestivamente all'Inps, a mezzo posta elettronica certificata, ogni variazione nell'assetto dei contratti collettivi di lavoro riferibili all'area di rappresentanza di Confindustria» ${ }^{147}$. Ciò vuol dire che - per come oggi configurato - il sistema di misurazione della rappresentatività, pur avendo adottato come "unità di misura" i perimetri dei Ccnl, non priverebbe le or-

145 Così, F. Scarpelli, Il Testo Unico sulla rappresentanza tra relazioni industriali e diritto, cit., pp. 693-694.

146 V. LECCESE, La contrattazione collettiva nazionale oggi: caratteri, metamorfosi e criticità. Temi per il dibattito, in A. Lassandari, F. Martelloni, P. TuldiNI, C. Zoli (a cura di), La contrattazione collettiva nello spazio economico globale, cit., p. 113.

147 In precedenza, erano state stipulate il 16 marzo 2015 una convenzione tra Inps, Confindustria, Cgil, Cisl e Uil, e il 16 marzo 2016 una convenzione tra Inps, Confservizi, Cgil, Cisl e Uil. Dopo la convenzione citata nel testo del 10 settembre 2019, ne è stata stipulata un'altra il 27 settembre 2019 tra Inps, Inl, Confapi, Cgil, Cisl e Uil. 
ganizzazioni stipulanti della prerogativa originaria di stabilire il campo di applicazione dei contratti collettivi, ma imporrebbe loro, in tali eventualità, un obbligo di comunicazione, volto a conferire "trasparenza" e "pubblicità" alle relative determinazioni, allo scopo di non alterare i meccanismi di rilevazione e ponderazione dei dati.

La seconda critica solleva il problema che la verifica di rappresentatività sarebbe, comunque, interna alle aree di rappresentanza del circuito confederale (meglio, di alcune confederazioni dei datori di lavoro) senza poter raggiungere la profondità di intervento e la vincolatività erga omnes proprie della legge. Anche se resa operativa, cioè, la verifica non potrebbe impedire a soggetti dissenzienti di realizzare comportamenti ostruzionistici o di neutralizzarne, comunque, gli esiti, mediante la creazione di nuove categorie contrattuali.

Tali dubbi sono, in qualche modo, condivisibili148, benché alcune chiose possano contribuire a ridimensionarne in concreto la portata. Non serve dimostrazione del fatto che la legge abbia una capacità di sanzione dei rapporti civili e sociali che il contratto non raggiunge, per lo meno dalla modernità giuridica in avanti ${ }^{149}$. Tuttavia, da un lato, ciò non significa che sia la legge a cogliere sempre in modo più appropriato le dinamiche in atto nella società e che il decisore pubblico possa stabilire meccanismi di razionalizzazione del pluralismo più affidabili di quelli che l'autonomia collettiva sia in grado di darsi ${ }^{150}$. Soprattutto, dall'altro lato, non si può trascurare che, nonostante il fervore di questi anni ${ }^{151}$, non sembra che si siano create le condizioni per l'emanazione da parte del Parlamento di una legge sindacale.

Non senza pragmatismo, giova ammettere che non sembrano esservi all'orizzonte alternative rispetto alla valorizzazione delle potenzialità espansive che un meccanismo - rodato e ben congegnato - di verifica della rappresentatività sindacale può avere, sia

148 Una sintetica esposizione in B. CARUSO, Testo Unico sulla Rappresentan$z a$, in WP CSDLE “Massimo D’Antona”.IT, 226/2014, pp. 10-13.

149 G. VArdaro, Contrattazione collettiva e sistema giuridico, cit., p. 102.

150 È il cuore della lezione di M. GRANDI, In difesa della rappresentanza sindacale, cit., p. 640.

151 Infra, capitolo III, $\S 3$. 
nel sistema di relazioni industriali sia nell'ordinamento giuridi$\mathrm{co}^{152}$.

Una volta (meglio, se) a regime, tali meccanismi dovrebbero consentire di verificare quali siano i soggetti più accreditati, dal lato dei lavoratori e, in prospettiva, dei datori di lavoro ${ }^{153}$, a stabilire le discipline collettive di lavoro in relazione alle diverse tipologie di attività. Da ciò potrebbero derivare alcuni effetti positivi per il funzionamento del sistema contrattuale.

Il fatto di dotarsi di congegni di misurazione della rappresentatività sindacale imporrebbe per prima cosa di stabilire quali siano gli ambiti nei quali operare la misurazione (come già più volte rilevato); poiché i recenti accordi hanno individuato nei perimetri dei contratti collettivi tali unità, parrebbe che la determinazione nel conseguire il primo obiettivo (la misurazione della rappresentatività) possa essere di stimolo anche per il raggiungimento del secondo (la mappatura dei perimetri contrattuali, che costituisce il presupposto per una semplificazione del quadro negoziale) ${ }^{154}$.

Inoltre, il fatto di rendere operativi i congegni di misurazione della rappresentatività sindacale dovrebbe contribuire, sia pure indirettamente, a contrastare i fenomeni di proliferazione di contratti collettivi di categoria ${ }^{155}$. Nella prospettiva interna al circuito confederale diverrebbe più complesso, da un punto di vista politico-sindacale, sconfinare nella sfera di "giurisdizione" di altre

152 Cfr. G. Ferraro, Sul rinnovato "sistema" di relazioni industriali, in L. Zoppoli, A. Zoppoli, M. Delfino (a cura di), Una nuova Costituzione per il sistema di relazioni sindacali?, cit., p. 302 ma passim.

153 Come osserva M. Ricci, L'accordo interconfederale del 9 marzo 2018: una svolta dagli esiti incerti, cit., pp. 1400-1401, 1412.

154 Questa correlazione si trae da tutti i più recenti accordi interconfederali, ma in modo particolarmente evidente dal Patto per la fabbrica del 2018 tra Confindustria e Cgil, Cisl e Uil (specialmente, punto 4 «democrazia e misura della rappresentanza»).

155 M. MarazZA, Dalla "autoregolamentazione" alla "legge sindacale"? La questione dell'ambito di misurazione della rappresentatività sindacale, cit., p. 621. L'autore ritiene tuttavia che i meccanismi elaborati con il Testo unico 2014 abbiano finito per avere l'effetto opposto e che anche per tale ragione si sia reso necessario il nuovo accordo interconfederale del 2018: M. MARAZZA, Perimetri e rappresentanze sindacali (dei datori di lavoro e dei lavoratori), cit., p. VIII. 
organizzazioni, senza potere, al contempo, in relazione a queste ultime, dimostrare un effettivo radicamento tra le imprese e tra i lavoratori. Nella prospettiva esterna, di contrasto al fenomeno della contrattazione "pirata", le operazioni di creazione ad hoc di contratti collettivi alternativi a quelli del circuito confederale diverrebbero, invero, meno credibili di quanto (talvolta) non lo siano (già) oggi. Né parrebbe peregrino rimarcare che il fatto di rendere operativi i meccanismi di misurazione della rappresentatività sindacale agevolerebbe le valutazioni che la giurisprudenza è tenuta ad effettuare nelle ipotesi di rinvio dalla legge all'autonomia collettiva ${ }^{156}$.

Nel processo di riforma del sistema contrattuale, la verifica di rappresentatività degli attori negoziali e la semplificazione dei contratti collettivi di categoria sono due lati di una stessa medaglia, utili allo scopo di irrobustire un sistema contrattuale indebolito dalle incursioni esterne dei contratti collettivi "pirata" e dai conflitti di giurisdizione interni.

In definitiva, l'esigenza di una «riconsiderazione della composizione quantitativa e qualitativa dei Ccnl» ${ }^{157}$ è parte integrante dell'agenda delle parti sociali. Gli accordi di area Confcommercio (24 novembre 2016) e Confesercenti ( 7 settembre 2017) ${ }^{158}$, con formulazioni identiche, auspicano «una riduzione dei molteplici

156 V. infra, cap. IV. È significativo che siano le stesse parti sociali a rimarcare questa correlazione tra riforma del sistema di relazioni sindacali e disposizioni di rinvio dalla legge ai contratti collettivi stipulati dai soggetti comparativamente più rappresentativi. V. ad esempio l'accordo interconfederale 24 novembre 2016 tra Confcommercio e Cgil, Cisl e Uil «per un nuovo sistema di relazioni sindacali e modello contrattuale» (in particolare, il punto sulla «rappresentanza»), l'accordo interconfederale 7 settembre 2017 tra Confesercenti e Cgil, Cisl e Uil (la premessa all'accordo) e l'accordo interconfederale 12 dicembre 2018 «sulle linee guida per la riforma delle relazioni industriali» tra Agci, Confcooperative, Legacoop, Cgil, Cisl e Uil (premessa all'accordo).

157 Così, il documento unitario del 14 gennaio 2016 di Cgil, Cisl e Uil su «Un moderno sistema di relazioni industriali», che prosegue: «Occorre per questo, sulla base delle reali esigenze di tutela di specifiche realtà produttive, conseguire un processo di razionalizzazione e diminuzione del numero di contratti nazionali, consapevoli che in parte essi rappresentano un processo di frammentazione della rappresentanza associativa dell'impresa».

158 Tra i due accordi interconfederali del 7 settembre 2017 di area Confesercenti si allude a quello su «relazioni sindacali». 
contratti collettivi esistenti, spesso insistenti sui medesimi settori/ comparti». In tali accordi si pone il punto di partenza del numero di contratti collettivi stipulati dalla confederazione per il comparto del Terziario di mercato (14 Ccnl Confcommercio e $8 \mathrm{Ccnl}$ Confesercenti) ${ }^{159}$ e si indica la volontà di dotarsi di strumenti di verifica della rappresentatività, sia delle organizzazioni dei lavoratori sia delle organizzazioni dei datori di lavoro, ma il processo è in fie$r i$. Nel comparto artigiano sono stati realizzati alcuni accorpamenti tra Ccnl (dopo gli accordi del 2009) ed altri sono stati previsti (accordo 23 novembre 2016), con l'obiettivo di arrivare ad accordi di settore per quattro macroaree (manifatturiero, servizi, edilizia e autotrasporto). In area Confindustria, il Patto per la fabbrica (2018) delinea un percorso in più fasi ${ }^{160}$, che, da una perimetrazione esatta della contrattazione collettiva (attività per la quale si rimanda alle analisi del Cnel) e da un'eventuale revisione delle unità negoziali, dovrebbe condurre all'attivazione dei meccanismi di misurazione della rappresentatività e, in prospettiva, al «rispetto dei perimetri della contrattazione»e a una maggiore «coerenza e funzionalità» della stessa ${ }^{161}$. In ciascuno degli accordi interconfederali il contrasto al dumping contrattuale è preso in considerazione come uno degli obiettivi centrali del processo di riforma delle relazioni industriali ${ }^{162}$. La soluzione, comunque, non è vicina ${ }^{163}$. Per un verso, la scissione del sistema confederale in tante aree quante sono le principali confederazioni dei datori di lavoro è eloquente della lontananza, non solo temporale, dagli accordi "costituenti"

159 Tra i due accordi interconfederali del 7 settembre 2017 di area Confesercenti si allude adesso a quello su «modello contrattuale».

160 M. Ricci, L'accordo interconfederale del 9 marzo 2018: una svolta dagli esiti incerti, cit., p. 1399.

161 L. Bordogna, L'accordo Confindustria-sindacati del 9 marzo 2018 su relazioni industriali e contrattazione collettiva, cit., pp. 46-47.

162 Oltre agli accordi menzionati nelle note precedenti v. (anche per la particolare rilevanza che il fenomeno della contrattazione "pirata" ha assunto nel settore della cooperazione) il più volte citato accordo interconfederale 12 dicembre 2018 «sulle linee guida per la riforma delle relazioni industriali».

163 F. Liso, Qualche erratica considerazione sul recente accordo interconfederale Confindustria, Cgil, Cisl e Uil del 9 marzo 2018, cit., p. 5. 
del $1993^{164}$. Per l'altro, il processo delineato dalle Confederazioni rischia, comunque, di arrestarsi, se non implementato dalle federazioni di categoria in sede di rinnovi dei $\mathrm{Ccnl}^{165}$.

164 A. LASSANDARI, Sulla verifica di rappresentatività delle organizzazioni sindacali datoriali, cit., p. 5.

165 P. Tosi, Concorrenza, lavoro, diritti (aspetti collettivo-sindacali), cit., pp. $1340-1341$. 



\section{CAPitolo III}

\section{AUTONOMIA DELLE PARTI ED INTERVENTO DELLA LEGGE NELLA DEFINIZIONE DELLE UNITÀ NEGOZIALI}

SOMMARIO: 1. Libertà di definizione delle unità negoziali e certezza giuridica: considerazioni sull'esigenza di pervenire ad un contemperamento. -2 . Il criterio oggettivo di collegamento tra le unità negoziali e i rapporti di lavoro. - 3. I meccanismi di definizione delle unità negoziali: una possibile bipartizione. - 3.1. Meccanismi antecedenti (che operano in una fase che precede lo svolgimento della contrattazione collettiva). - 3.2. Meccanismi contestuali (in cui la categoria è fissata con la stipulazione dei contratti collettivi). - 3.3. Considerazioni conclusive. -4 . Rassegna delle soluzioni tecniche avanzate per la definizione delle unità negoziali nelle proposte di attuazione dell'art. 39 Cost. - 4.1. Dall'entrata in vigore della Costituzione ai primi anni Sessanta. - 4.2. Riprende il dibattito sulla legge sindacale: gli anni Ottanta e Novanta. - 4.3. La Carta dei diritti della Cgil e le proposte della rivista $D L M$ e del gruppo Freccia Rossa. - 5. La definizione dei comparti di contrattazione nel settore pubblico. - 5.1. Evoluzione delle discipline legislative e della corrispondente attività negoziale. - 5.2. La limitata "sovranità" delle organizzazioni sindacali nella definizione dei comparti. 6. Sciopero nei servizi pubblici essenziali e prestazioni indispensabili. - 6.1. Il quadro normativo: cenni essenziali. - 6.2. La definizione del campo di applicazione degli accordi collettivi.

1. Libertà di definizione delle unità negoziali e certezza giuridica: considerazioni sull'esigenza di pervenire ad un contemperamento

Nelle ipotesi in cui il contratto collettivo trova per legge applicazione erga omnes ${ }^{1}$, è possibile che si debba pervenire altresì

1 Ciò può avvenire in una varietà di soluzioni tecniche: un quadro comparato in S. Hayter, J. Visser (eds), Collective Agreements: Extending Labour Protection, Geneva, International Labour Organization, 2018. 
ad un contemperamento tra la "originaria" libertà delle parti sociali di definire in autonomia le unità negoziali e le esigenze di certezza dei rapporti giuridici che derivano dall'intervento eteronomo in questa materia. In tal caso, la definizione delle unità negoziali della contrattazione collettiva non sarà (più) soltanto espressione di una sfera di "competenza" o, se si vuole, di "rilevanza sociale" delle organizzazioni sindacali e dei datori di lavoro nel sistema di relazioni industriali: le tipizzazioni operate dai contratti collettivi diverranno, altresì, giuridicamente rilevanti, allo scopo di individuare un'area di obbligatorietà delle discipline di lavoro, che normalmente non coinciderà, se non in parte (specialmente nei segmenti più periferici del mercato), con quella di rappresentanza delle associazioni firmatarie $^{2}$.

Si tratta di considerazioni che nella loro sostanza sono acquisite da decenni alla riflessione giuridica ${ }^{3}$ : una contrattazione collettiva con effetti maggiori di quelli ricavabili dal diritto comune esige non soltanto «qualche garanzia sulla formazione e sulla qualificazione giuridica dei soggetti ${ }^{4}$, ma anche di misurarsi con l'inquadramento delle categorie contrattuali ${ }^{5}$. È lo «scotto che paga il sindacato nel momento in cui [...] permette allo Stato di invadere la zona di riservato dominio che gli è propria, sia pure per consolidare su di un'area geografica o per una professione più estesa i risultati della sua vocazione contrattuale e rivendicativa ${ }^{6}$.

2 S. Liebman, Contributo allo studio della contrattazione collettiva nell'ordinamento giuridico italiano, Milano, Giuffrè, 1986, p. 70.

3 Tra i primi a interrogarsi in modo approfondito su tali correlazioni, G. PERA, Problemi costituzionali del diritto sindacale italiano, cit., pp. 51-68.

4 Così, M. Grand, Normatività privata e contratto collettivo, in AA.VV., Il sistema delle fonti nel diritto del lavoro. Atti delle giornate di studio di diritto del lavoro Foggia-Baia delle Zagare, 25-26 maggio 2001, Milano, Giuffrè, 2002, p. 218.

5 M. Ricci, Il contratto collettivo fonte e l'art. 39 Cost., in AA.VV., Il sistema delle fonti nel diritto del lavoro, cit., p. 143.

6 Così, B. Veneziani, Stato e autonomia collettiva. Diritto sindacale comparato, cit., p. 177. Da tempo si è rilevato che l'intervento della legge ha lo scopo di rafforzare il tasso di applicazione dei contratti collettivi nei settori più marginali del mercato, nei quali le organizzazioni dei datori di lavoro e dei lavoratori hanno una "presa" minore, rispettivamente, sul tessuto produttivo e sulla forza lavoro: G. GIUGNI, La validità "erga omnes” dei contratti collettivi, cit., p. 69 ss. 
Naturalmente è comprensibile che, in base alle vicende storiche delle relazioni industriali, oltre che tra i diversi ordinamenti giuridici, possa variare, anche in modo significativo, il punto di equilibrio tra autonomia ed eteronomia da considerarsi ottimale o, per lo meno, accettabile per le parti sociali, nella definizione delle unità contrattuali ${ }^{7}$.

Vi è comunque un punto fermo: dotarsi di meccanismi con cui pervenire ad una definizione delle unità negoziali non equivale a dover fissare, una volta per tutte, la disposizione degli "insiemi di giurisdizioni” che compongono il sistema di contrattazione collettiva. In questo settore della vita civile sarebbe impensabile non lasciare alle parti sociali la possibilità di rivedere, nel tempo, le proprie determinazioni, in ordine alle sfere di influenza organizzativa e negoziale ${ }^{8}$. Diversamente, si finirebbe per imporre loro una "camicia di nesso", che paralizzerebbe la contrattazione collettiva, soprattutto nelle fasi caratterizzate da rapidi mutamenti sociali, tecnologici e dei processi produttivi: ma non è questo un elemento strutturale delle società che vengono dette ad economia avanzata? ${ }^{9}$.

7 U. CARABELLI, Legge sindacale e ordinamento intersindacale: alcune riflessioni sparse, in AA.VV., L'attuazione degli articoli 39 e 46 della Costituzione. Tre proposte a confronto, Quaderni Riv. Giur. Lav., Roma, Ediesse, 2016, p. 184.

8 È significativo che ciò si possa rimarcare anche per l'ordinamento corporativo, nonostante l'adozione di meccanismi di predeterminazione coatta delle categorie contrattuali e di attribuzione, ad una sola organizzazione, della rappresentanza legale di ciascuna di esse: il sistema conobbe, a più riprese, specie negli anni Trenta, operazioni di rimaneggiamento e di manipolazione delle categorie: $\mathrm{cfr}$. in generale sul tema G. Proia, L'inquadramento dei datori di lavoro, cit., cap. II.

9 È un luogo comune, ma alle volte torna utile ricordarsi della «mentalità particolarmente duttile del sindacalista, che non è mai a corto di espedienti e di adattamenti (anche se - talvolta - non del tutto ortodossi, dal punto di vista giuridico), per raggiungere la finalità che si propone; cioè, l'equo accordo con la controparte». Le citazioni sono tratte da alcuni passaggi dedicati al tema della categoria professionale, da un documento risalente, ma di indubbio valore (non soltanto storico): le Risposte al questionario del Ministro del lavoro e della previdenza sociale sul tema Verso la nuova legislazione sindacale, pubblicate dalla Riv. Dir. Lav., 1949, III, p. 94 ss., specie, per le citazioni, p. 97 (si trattava qui delle risposte della Cgil, che respingeva all'epoca ogni ipotesi di intervento rigido dei pubblici poteri sul tema delle categorie professionali). 
2. Il criterio oggettivo di collegamento tra le unità negoziali e $i$ rapporti di lavoro

Affrontare il problema della ripartizione del sistema contrattuale in unità negoziali implica porsi quello del collegamento tra gli ambiti di applicazione dei contratti collettivi e i rapporti di lavoro. Nella sostanza, occorre individuare taluni criteri che consentano di individuare con ragionevole certezza quali discipline collettive siano applicabili ai rapporti di lavoro, tra le diverse che potrebbero essere potenzialmente richiamabili (più un sistema contrattuale diviene complesso e articolato, più tale selezione può in concreto farsi complicata). In altri termini, se, da una parte, il sistema contrattuale dovrebbe essere suddiviso in sfere di competenza negoziale (quali che siano i meccanismi a tale scopo adottati: v. infra, § 3 ss.), dall'altra occorrerebbe comunque stabilire in base a quali criteri le parti dei rapporti individuali di lavoro siano associate ad una o ad un'altra di tali sfere. Un principio basilare di civiltà giuridica sembra suggerire che, nel caso in cui il contratto collettivo spieghi i propri effetti al di là dei soggetti aderenti alle organizzazioni stipulanti, non possano esservi eccessive ambiguità su quale sia quello applicabile, in quanto le parti dei rapporti di lavoro sono tenute a rispettare obblighi aggiuntivi rispetto a quelli che deriverebbero dall'adesione volontaria al sistema contrattuale.

In astratto, i criteri utilizzati per selezionare la disciplina collettiva applicabile ai rapporti di lavoro potrebbero essere i più vari. Non è escluso, ad esempio, che possano consistere nell'inquadramento delle parti dei rapporti di lavoro nelle organizzazioni stipulanti, ma è evidente che occorrerebbe a tal fine dotarsi di un sistema di sindacalismo obbligatorio ${ }^{10}$. Escluso il criterio

10 Vigente l'ordinamento corporativo in Italia era stata presa in considerazione, nei lavori per la redazione del codice civile del 1942, la possibilità di stabilire come criterio di collegamento quello dell'inquadramento soggettivo. Tale eventualità era stata tuttavia scartata, in sede di redazione del codice, in favore del criterio oggettivo poi stabilito all'art. 2070 c.c., considerato più affidabile e rispettoso delle posizioni giuridiche delle parti dei rapporti di lavoro. V. sul punto la Relazione del Ministro Guardasigilli Grandi al c.c. del 1942, n. 817; un cenno, dal quale ho tratto lo spunto, in P. Bellocchi, Art. 2070, in G. Amoroso, V. Di Cerbo, A. Maresca (a cura di), Diritto del lavoro, 1, La Costituzione, il Codice ci- 
dell'inquadramento sindacale, logica vuole che, per la contrattazione di settore, i criteri di collegamento consistano prevalentemente nell'attività svolta dai datori di lavoro. Ciò si deve al fatto che nella gran parte dei sistemi di relazioni industriali (nei quali è diffusa la negoziazione multi-employer) la contrattazione collettiva sia prevalentemente organizzata sulla base di un sindacalismo cd. industriale ${ }^{11}$.

Anche nel sistema italiano, che riconosce piena libertà alle parti di definire le unità negoziali, i contratti collettivi, sia pure con tecniche di redazione che cambiano da settore a settore, delineano tendenzialmente le aree contrattuali con riferimento alle tipologie di attività espletate dalle imprese ${ }^{12}$, allo scopo di garantire uniformità alle discipline del lavoro tra le multiformi realtà aziendali (non ultimo, a tutela della tenuta della stessa aggregazione sindacale $)^{13}$.

Un criterio oggettivo basato sull'attività svolta dai datori di lavoro era stato adottato dal diritto corporativo (art. 2070 c.c. $)^{14}$. La sua applicazione ai contratti collettivi di diritto comune è stata esclusa dalle Sezioni Unite della Cassazione con la sentenza n. 2665/1997, quale coerente (benché tardiva) conseguenza dell'o-

vile e le leggi speciali, Milano, Giuffrè, 2017, p. 662. Cfr. altresì M. NovELLA, Applicazione del contratto collettivo e categoria professionale, cit., il quale illustra come la redazione dell'art. 2070 c.c. sia stata il risultato di un dibattito avviatosi con la legge del '26 e composto dalla giurisprudenza di legittimità soltanto nei primi anni Quaranta: in base all'orientamento risultato prevalente, anche nel vigore della legge del '26 il criterio dell'attività svolta dal datore di lavoro avrebbe prevalso, ai fini dell'applicazione dei contratti collettivi, su quello eventualmente difforme dell'inquadramento sindacale.

11 Per il caso italiano v. in tal senso M. Rusciano, Il contratto collettivo, in Impresa e lavoro, T. I, $2^{\circ}$ ed., in P. Rescigno (diretto da), Trattato di diritto privato, Torino, Utet, 2004, p. 45. Cfr. altresì G. Giugni, Introduzione a S. Perlman, Per una teoria dell'azione sindacale, Roma, Edizioni lavoro, 1980, pp. 29-30, 34-35.

12 Qualche esempio lo si è fornito supra, cap. I, § 5. In dottrina cfr. R. ScoGNAmiglio, Il problema dell'applicabilità dell'art. 2070 c.c.: categoria e contratto collettivo, in Scritti in onore di Giuseppe Federico Mancini, I, Diritto del lavoro, Milano, Giuffrè, 1998, p. 570.

13 Cfr. M.G. Garofalo, Solidarietà, differenze, riunificazione del mondo del lavoro (1991), in M. BARBIERI, R. Voza (a cura di), Gianni Garofalo. Il pane del sapere, Roma, Ediesse, 2011, p. 297.

14 V. supra nota 10; amplius, L. Riva Sanseverino, Della impresa: disciplina delle attività professionali. Impresa in generale, II ed., Bologna-Roma, ZanichelliSocietà editrice del Foro italiano, 1956, p. 70 ss. 
rientamento sull'efficacia soggettivamente limitata dei contratti collettivi ${ }^{15}$. In sistemi nei quali la contrattazione collettiva acquisisce normalmente efficacia generale, analoghi criteri sono previsti dalla legge o adottati dalla giurisprudenza: è il caso ad esempio della Francia ${ }^{16}$ o del Belgio ${ }^{17}$.

Vero è che l'adeguatezza di un siffatto criterio oggettivo può essere messa in discussione, nel contesto di un'economia di servizi, nella quale i processi produttivi sono trasversali rispetto alle ripartizioni tradizionali della contrattazione collettiva, ma non è questo un tema che può essere adeguatamente approfondito ${ }^{18}$. Vale la pena invece precisare che esso è in sé neutrale rispetto al principio di libertà sindacale. Al proposito, non vi è migliore dimostrazione di quella fornita, per la quale disposizioni simili sono state adottate sia in un modello sindacale autoritario (come quello italiano del ventennio), sia da regimi di Paesi europei (Belgio o Francia, ad esempio) in cui non è in discussione la libertà sindacale e che sono, per di più, dotati di congegni eterogenei per la definizione delle unità negoziali (v. infra, § 3). Per un verso, infatti, il criterio oggettivo non interferisce con i meccanismi di definizione delle categorie ${ }^{19}$ : esso non ha «alcun riflesso sulla libertà di autodetermi-

15 Cass SSUU n. 2665/1997, cit. La questione è stata approfondita supra, cap. II, § 2 .

16 Art. L2261-1 code du travail: «La convention collective applicable est celle dont relève l'activité principale exercée par l'employeur».

17 Nell'ordinamento belga, la legge del 5 dicembre 1968 sulla contrattazione collettiva è interpretata dalla giurisprudenza nel senso di prevedere che l'applicazione dei contratti collettivi sia stabilita in base all'attività prevalente esercitata dal datore di lavoro sul territorio belga (cfr. V. VANNES, Identification de la commission paritaire compétente envers les entreprises concernées, in Orientations, 2010, 4, p. 1 ss.).

18 Cfr. il Documento unitario del 14 gennaio 2016 Cgil, Cisl, Uil per «Un moderno sistema di relazioni industriali», parte I «La contrattazione: riformare il modello per renderlo più inclusivo».

19 V. in questo senso C. cost. n. 105/1969, cit., sull'applicabilità dell'art. 2070 c.c. ai contratti collettivi recepiti nei decreti delegati ex 1. n. 741/1959, nella quale si afferma che «L'art. 2070 cod. civ. risulta, pertanto, diretto a dirimere razionalmente gli eventuali conflitti tra diverse normative collettive, di cui sia invocata l'applicazione ad un rapporto individuale di lavoro, e non è in contrasto col principio della libertà ed autonomia sindacale garantito dall'art. 39, primo comma, della Costituzione, in quanto ha riferimento alla nozione di categoria professionale risultante dalla spontanea organizzazione sindacale e dall'autonomia collettiva». Cfr. 
nazione delle unità organizzative e contrattuali», che interviene (e si conclude) in un momento precedente rispetto a quello in cui si pone la questione dell'applicazione dei contratti collettivi ai rapporti individuali di lavoro ${ }^{20}$. Per l'altro, esso non si pone neppure in collisione con la libertà delle parti dei rapporti di lavoro, atteso che «presuppone, e non impone, la soggezione dei singoli alla disciplina collettiva ${ }^{21}$.

sul punto D. GUERrIERI, I vigenti decreti di applicabilità del contratto collettivo, in Mass. Giur. Lav., 1969, p. 389, ma passim. Nella giurisprudenza di legittimità, per l'applicabilità dell'art. 2070 c.c. ai contratti collettivi recepiti nei decreti delegati, v. Cass. 4 febbraio 1989, n. 701, in Riv. It. Dir. Lav., 1990, II, p. 61, nota A. VAlLeBONA, Cass. n. 6412/1993, cit.

20 Così, P. Bellocchi, Art. 2070, cit., p. 665. Per quanto fosse viziata dal noto errore di fondo risolto dalle Sezioni Unite della Cassazione nel 1997, di ciò è una prova la stessa giurisprudenza che affermava l'applicabilità dell'art. 2070 c.c. ai contratti collettivi di diritto comune (per una rassegna cfr. M. Quaranta, Art. 2070 , in R. De Luca Tamajo, O. Mazzotta (a cura di), Commentario breve alle leggi sul lavoro, VI ed., Milano, Wolters Kluwer-Cedam, 2018, p. 497 ss.). Infatti, se si eccettuano isolati e risalenti precedenti di merito (App. L'Aquila 5 marzo 1963, in Orient. Giur. Lav., 1963, p. 350, o Trib. Parma 21 gennaio 1965, in Riv. Giur. Lav., 1965, II, p. 211) o, a tutto concedere, obiter dicta di sentenze più recenti, tale giurisprudenza non ha mai messo in discussione la prerogativa delle parti sociali di delineare gli ambiti di applicazione dei contratti collettivi: neanche quando, in alcuni settori, sono state sperimentate soluzioni eccentriche rispetto alle tradizionali aree contrattuali, per far fronte alle cangianti geografie produttive e al decentramento delle attività d'impresa, negli anni Ottanta e Novanta del secolo scorso (cfr. Cass. 23 novembre 1984, n. 6063, in Riv. It. Dir. Lav., 1985, II, p. 593 ss., nota M. PAPAleoni, Cass. 19 febbraio 1986, n. 1005, in Giust. Civ., 1986, p. 1669 ss., nota M.P., P. Roma, 2 febbraio 1990, in Riv. It. Dir. Lav., 1991, II, p. 504 ss., nota L. Angelini, Cass. 30 gennaio 1992, n. 976, in Riv. It. Dir. Lav., 1992, II, p. 531 ss., nota M. CARO; ulteriori riferimenti in R. BORTONE ( $\mathrm{P}$. CuRzIO), Il contratto collettivo, Torino, Utet, 1984, pp. 192-193). Quella giurisprudenza era volta, per lo più, a individuare la retribuzione conforme all'art. 36 Cost., ricavandola dal contratto collettivo di settore stipulato per quel tipo di attività d'impresa. Ciò, per i casi in cui i datori di lavoro non applicassero alcun contratto collettivo, oppure, più di frequente, in cui applicassero un contratto collettivo "innaturale" rispetto all'attività svolta. Si perveniva, così, a selezionare la disciplina economica più favorevole al lavoratore (P. Bellocchi, Art. 2070, cit., p. 665), in un'ottica di tutela individuale, ma con il risultato, indiretto, di «delineare un quadro legale minimo di sostegno della contrattazione in assenza di una disciplina legislativa del contratto» (così, A. VISCOMI, Soggettività sindacale e categorie contrattuali, cit., p. 73).

21 Così, P. Bellocchi, Art. 2070, cit., p. 662. 
3. I meccanismi di definizione delle unità negoziali: una possibile bipartizione

Muovendo sempre dal principio di libertà sindacale (ossia, escludendo l'ipotesi di un ordinamento autoritario dei rapporti collettivi di lavoro), sono pensabili molteplici soluzioni tecniche, mediante le quali pervenire ad una ripartizione in unità negoziali del sistema di contrattazione collettiva.

Si proporrà il seguente criterio di classificazione, con il quale distinguere a seconda che le unità contrattuali siano definite: $a$ ) in una fase che precede la stipulazione dei contratti collettivi, con una determinazione per così dire separata rispetto alla vera e propria trattativa negoziale; $b$ ) contestualmente alla conclusione dei contratti collettivi, con una determinazione adottata nel medesimo testo che pone le discipline di lavoro. Si illustreranno alcuni esempi dell'uno e dell'altro tipo, traendoli dal diritto italiano o da altri ordinamenti giuridici.

3.1. Meccanismi antecedenti (che operano in una fase che precede lo svolgimento della contrattazione collettiva)

Tra le soluzioni del tipo a) potrebbe rientrare quella prevista “in potenza” dall'art. 39, c. 4, Cost. La norma costituzionale - è il cuore della prolusione bolognese di Mancini del 1962 - ha ripreso dal diritto corporativo, sia pure depurandola dalle "incrostazioni più autoritarie", la soluzione di una determinazione ex ante delle unità negoziali della contrattazione collettiva (la categoria che precede la contrattazione collettiva, nelle parole del chiaro autore $)^{22}$. In particolare, ciò si sarebbe realizzato sia mediante la registrazione delle associazioni sindacali per categorie di inquadramento (cioè, con una razionalizzazione dei soggetti), sia e soprattutto con l'istituzione delle rappresentanze unitarie, atteso che si sarebbe in primis dovuto stabilire per quali unità di negoziazione costituirle. Non ci si

22 La profonda influenza esercitata sulla riflessione successiva dalle tesi di Mancini del 1962 è ravvisabile anche nella dottrina più recente: cfr. P. IcHINO, $L a$ crepa interna all'art. 39, cit. 
può nascondere, tuttavia, che in assenza di una legge attuativa della norma costituzionale ogni affermazione sul punto sia controvertibi$1 \mathrm{e}^{23}$ : molto se non tutto dipenderebbe (o sarebbe dipeso), cioè, dalle soluzioni tecniche adottate in sede di attuazione. Occorrerà perciò approfondire la questione (v. infra, § 4) con una rassegna delle soluzioni tecniche avanzate per la definizione delle unità negoziali nelle proposte di attuazione dell'art. 39 Cost.

Nell'ordinamento giuridico italiano un secondo esempio di determinazione preventiva delle unità negoziali si può trarre dalle discipline vigenti in materia di contrattazione collettiva per il lavoro pubblico. Si ricava dagli artt. 40 c. 2, 41 c. 5 e 47, d.lgs. n. 165/2001, che la definizione dei comparti di contrattazione collettiva e delle aree separate per la dirigenza è realizzata, in vista dei rinnovi contrattuali, mediante la stipulazione di accordi quadro tra Aran e confederazioni rappresentative. L'architettura del sistema negoziale ${ }^{24}$ è rimessa dalla legge alle determinazioni congiunte dell'Aran (che su tali profili agisce su indirizzo collegiale dei comitati di settore: art. 41, c. 5) e delle "principali" (art. 43, c. 4) confederazioni dei lavoratori. Tali meccanismi sollevano rilevanti questioni, che saranno illustrate infra $\S 5$.

Dal diritto comparato è possibile trarre almeno altri due esempi di meccanismi riconducibili alle tipologie $s u b a$ ). Il primo di essi si ricava dall'ordinamento statunitense. Il National labour relations $a c t$ (sec. 9, lett. $a-b$ ) prevede che i sindacati possano ottenere una rappresentanza monopolistica dei lavoratori, anche a fini negoziali, per ciascuna bargaining unit considerata «appropriate», in caso di esito favorevole di una consultazione elettorale indetta tra i lavoratori ad essa addetti. È noto che tale sistema difficilmente può consentire di organizzare una consultazione e, perciò, una contrattazione collettiva multi-employer, ma non è questo il punto che qui inte-

${ }^{23}$ Il che equivale a dire che in questa materia i nodi di fondo non sono mai stati sciolti: M. Rusciano, Introduzione. Che fare dell'art. 39 della Costituzione?, in L. Zoppoli, A. Zoppoli, M. Delfino (a cura di), Una nuova Costituzione per il sistema di relazioni sindacali?, cit., p. 439 ss.

24 R. Salomone, Rappresentatività sindacale, ambiti negoziali e procedure nella contrattazione collettiva del pubblico impiego, in Dir. Lav. Merc., 2014, p. 500. 
ressa. Nella dottrina italiana, questo meccanismo di definizione delle unità negoziali è stato studiato, sin dagli anni Cinquanta ${ }^{25}$, per la ragione che sembrava garantire una soluzione più democratica e agile di quella prevista dall'art. 39 Cost. $^{26}$. Vero è anche che, alla prova del tempo, la soluzione americana avrebbe mostrato limiti rilevanti. Da un lato, la possibilità per i datori di lavoro di contestare di fronte al National Labour Relations Board (NLRB) l'adeguatezza dell'unità contrattuale e, così, di ritardare (ed ostacolare, talvolta a lungo) il processo elettorale e l'avvio della contrattazione collettiva ${ }^{27}$. Dall'altro, l'attribuzione al $N L R B$ di «una responsabilità di cui forse il legislatore non aveva misurato interamente la portata ${ }^{28}$ : la determinazione circa il carattere «appropriate» dell'unità di contrattazione, sulla base, tra le altre cose, di una verifica circa la sussistenza tra i lavoratori di una comunanza di interessi tale da giustificare l'unicità del processo negoziale ${ }^{29}$.

Un secondo esempio si trae dall'ordinamento belga. Nel contesto di un sistema democratico-corporativo ${ }^{30}$, caratterizzato da un intervento ad ampio spettro della legge ${ }^{31}$, la contrattazione collettiva (di settore) è svolta dalle organizzazioni contrapposte in seno a commissioni paritetiche, la cui sfera di competenza è stabilita mediante

25 Per qualche breve spunto, v. G. PerA, L'obbligatorietà dei contratti collettivi di lavoro, in Il Ponte, 1959, pp. 42-43.

26 V. ad esempio V. CAssì, La rappresentanza unitaria dei sindacati registrati, in Riv. Dir. Lav., 1950, I, p. 192, nota 13 («Più larghi consensi ha invece il sistema vigente nell'America del Nord e che comporta le libere elezioni dei rappresentanti per la stipulazione dei contratti collettivi da parte di tutti i cittadini che vi sono interessati, indipendentemente alla loro iscrizione o meno ai sindacati»), ma per una trattazione più approfondita U. ProsPeretti, Lezioni di diritto sindacale comparato, Milano, Giuffrè, 1962, pp. 63 ss., 89.

27 L. Mariucci, Uno sguardo alle relazioni industriali negli USA, in Lav. Dir., 1987, pp. 80-81. Più di recente, M. FAIOLI, Sindacato statunitense, in Dig. Comm., Agg., 2015, p. 498 ss.

28 Così, G.F. MANcini, La rappresentanza sindacale nel diritto statunitense e l'art. 39 della Costituzione italiana, cit., p. 468.

29 Una recente rassegna delle decisioni del NLRB in Si. Bologna, La contrattazione collettiva negli Stati Uniti dal Wagner act ai nostri giorni, cit., p. 429 ss.

30 P. C. Schmitter, Still the Century of Corporatism?, in Rev. Pol., 1974, 36, 1, p. 93, ma passim.

31 F. Dorssemont, On the representative status of Belgian employers' organizations for the purpose of collective bargaining, in Dir. Lav. Rel. Ind., 2017, p. 253 ss. 
decreti reali, di competenza sostanziale del potere esecutivo (artt. 35-36, legge del 5 dicembre 1968) ${ }^{32}$. L'ambito di applicazione dei contratti collettivi dipenderà dalla sfera di competenza della commissione paritetica; tutti i datori di lavoro ed i lavoratori ricompresi in tale sfera saranno vincolati dal contratto collettivo, in caso di emanazione di un provvedimento amministrativo di estensione (art. 31 della medesima legge). Benché le prerogative dell'autorità statuale, in questa materia, siano tutt'altro che trascurabili, giova precisare che, nella sostanza, è dalla volontà degli attori sociali di organizzarsi e di negoziare accordi collettivi per un certo segmento della realtà socioeconomica che derivano l'esistenza e la sfera di competenza delle commissioni paritarie ${ }^{33}$. Al proposito, è significativo che i decreti reali con i quali si istituisce una commissione paritetica o se ne modifica la sfera di competenza siano emanati previa consultazione o su richiesta delle parti sociali.

\subsection{Meccanismi contestuali (in cui la categoria è fissata con la stipulazione dei contratti collettivi)}

È possibile selezionare alcuni esempi anche per quanto attiene alla tipologia di soluzioni del tipo $b$ ), nelle quali la definizione delle unità negoziali è svolta con la stipulazione stessa dei contratti collettivi.

Per l'ordinamento italiano, rientrava tra tali soluzioni quella adottata con 1. n. 741/1959. In assenza di disposizioni specifiche sul punto da parte della legge, la Corte costituzionale stabilì che il legislatore non avrebbe potuto modificare gli ambiti di applicazione dei contratti collettivi recepiti in decreto e che, pertanto, la loro efficacia obbligatoria avrebbe riguardato tutti i soggetti ricompresi nelle unità negoziali definite dai contratti collettivi ${ }^{34}$.

Una soluzione diversa, ma che ugualmente presuppone la libertà delle organizzazioni stipulanti di stabilire esse stesse il campo di appli-

32 Cfr. R. Blanpain, Labour law in Belgium, cit., pp. 367 ss., 392 ss.

33 F. Donssemont, On the representative status of Belgian employers' organizations for the purpose of collective bargaining, cit., p. 256. V. altresì K. VANDAELE, Belgium: stability on the surface, mounting tensions beneath, cit., p. 55.

34 La questione è stata illustrata supra, cap. I, § 6.1. 
cazione dei contratti collettivi, è delineata dalla 1. n. 146/1990, in materia di sciopero nei servizi pubblici essenziali. Le previsioni dei contratti collettivi, con le quali operare un contemperamento tra il diritto di sciopero e i diritti della persona costituzionalmente tutelati (art. 1, c. 1), sono sottoposte ad un controllo di idoneità della Commissione di garanzia (art. 13, lett. a)). Il tema sarà approfondito infra $\S 6$.

Alcuni esempi di soluzioni del tipo $b$ ) possono essere tratte anche dall'esperienza comparata. Vi rientrano anzitutto quelle adottate nell'ordinamento francese. In relazione alla contrattazione di settore, la legge prevede che il campo di applicazione dei contratti collettivi sia stabilito direttamente dalle organizzazioni stipulanti, sia pure con la precisazione che ciò debba avvenire in termini di attività economiche ${ }^{35}$. Il Ministro del lavoro può estendere, per decreto, l'obbligatorietà dei contratti collettivi per tutti i soggetti ricompresi nel relativo campo di applicazione; al ricorrere di alcune condizioni, l'obbligatorietà può essere disposta anche per porzioni di territorio o attività economiche non incluse negli ambiti di applicazione dei contratti collettivi, sovrapponendosi così alla originaria determinazione delle organizzazioni stipulanti (cd. élargissement) ${ }^{36}$.

Al pari di quelle tratte dal diritto USA, tali soluzioni normative sono state studiate dalla dottrina italiana fin dagli anni Cinquanta del secolo scorso, per la (identica) ragione che erano considerate più agili nelle forme e, nel complesso, più rispettose della libertà sindacale di quelle preconizzate dall'art. 39 Cost. ${ }^{37}$. Non si dice nulla di

35 Art. L2222-1, code du travail. Sul tema della definizione delle categorie contrattuali nell'ordinamento francese cfr. J. PORTA, La branche professionnelle: déconstruction et restructuration, in Dr. Ouvr., 2018, 842, p. 8.

36 Artt. L2261-15 e L2261-17, code du travail. Cfr. M. Magnani, Diritto sindacale europeo e comparato, Torino, Giappichelli, 2015, pp. 140-143, la quale sottolinea come tale eventualità sia stata spesso utilizzata dal potere esecutivo, più che per "espropriare" le parti sociali delle loro prerogative in materia di determinazione del campo di applicazione dei contratti collettivi, allo scopo di sollecitarle a negoziare su ambiti economici e/o territoriali non ancora "coperti" dalla contrattazione collettiva.

37 Tra i molti, v. G.F. MANCINI, La rappresentanza sindacale nel diritto statunitense e l'art. 39 della Costituzione italiana, cit., pp. 462-463, menzionava rapidamente la soluzione francese, pur escludendo la possibilità di adottare un meccanismo simile, «in quanto del tutto in contrasto colla lettera dell'art. 39»; vedi alcuni anni dopo G. Pera, L'obbligatorietà dei contratti collettivi di lavoro, cit., pp. 42-43, U. Prosperetti, Lezioni di diritto sindacale comparato, cit., p. 37 ss. 
nuovo, se si osserva che la stessa soluzione adottata con la legge Vigorelli nel 1959 era ispirata dall'esperienza francese. Tuttavia, può essere utile qualche ulteriore precisazione, in considerazione delle novità sopraggiunte da alcuni anni. Nel contesto di una rivisitazione profonda del diritto sindacale ${ }^{38}$, il legislatore ha avviato (legge del 5 marzo 2014, ma con intensità crescente nelle riforme successive), un processo di ristrutturazione delle unità negoziali della contrattazione collettiva (vi sono ora dedicati gli artt. dal 2261-32 al 226134 del code du travail) ${ }^{39}$. Ciò dovrebbe portare ad una drastica riduzione del numero di contratti collettivi: negli auspici, da oltre 900 a circa $200^{40}$. Sia pure sollecitando in prima battuta le parti sociali a provvedere esse stesse ad una ristrutturazione delle unità negoziali (per via di accorpamenti, dismissioni di unità non più operative ed altri accorgimenti), le riforme hanno, a questi fini, attribuito amplissime prerogative al Ministro del lavoro. Per ragioni di «intérêt général» in sostanza connesse al buon funzionamento del sistema contrattuale, l'autorità pubblica ha estesi margini di manovra, soprattutto in caso di assenza o di stallo delle negoziazioni, per procedere d'imperio con la ristrutturazione della struttura contrattuale, in antitesi rispetto all'esperienza invalsa nel sistema francese, orientata a riconoscere piena libertà alle parti stipulanti nella definizione del campo di applicazione dei contratti collettivi ${ }^{41}$.

38 Un quadro complessivo in E. Perskine, C. Wolmark, Droit du travail: 2019, Paris, Dalloz, 2018.

39 La riforma era stata preceduta alcuni anni prima dalla redazione di un rapporto (2008), commissionato dal Primo ministro, nel quale una delle conclusioni tratte da uno studio dettagliato della contrattazione collettiva di settore era proprio la opportunità di una semplificazione del sistema contrattuale: cfr. J.-F. PoIsson, Rapport sur la négociation collective et les branches professionnelles, cit.

40 Cfr. S. NAdal, La restructuration des branches professionnelles: réflexions sur une mutation forcée, in Dr. Soc., 2016, p. 110 ss., ID., Governance du niveau et des règles de branche: les nouveaux visages de l'emprise étatique, in Rev. Dr. Trav., 2017, p. 652 ss., J. PoRTA, La branche professionnelle: déconstruction et restructuration, cit. Cfr. anche il numero 11/2018 di Droit Social, con interventi di P.-H. Antonmattei, B. Redt et al., Ph. Portier, P. Voisine, G. Lécuelle, M. Morand, G. VACHET.

41 Salvo che per alcuni profili minori, il Conseil constitutionnel ha ritenuto che tali prerogative non violassero i diritti costituzionali (in particolare, art. 4 della Dichiarazione dei diritti dell'uomo e del cittadino del 1789 in materia di libertà contrattuale e alinea 8 del Preambolo alla Costituzione del 1946 in materia di par- 
Un secondo esempio da ricondurre al tipo $b$ ) si trae dall'ordinamento spagnolo. L'art. 83.1, estatuto de los trabajadores prevede che l'ambito di applicazione dei contratti collettivi sia stabilito per accordo delle parti ${ }^{42}$. La legge si limita a prevedere che $\mathrm{i}$ contratti collettivi cd. statutari debbano definire in modo espresso il proprio ambito di applicazione, dal punto di vista personale, funzionale, territoriale e temporale (art. 85.3, lett. $b$ ). Se si considera la storia recente del Paese, non può che apparire significativo che il Tribunal Constitucional abbia rimarcato come la libertà di definire le unità di negoziazione sia uno degli elementi che distingue tale meccanismo da «quel che avviene in un regime di tipo corporativo o di segno autoritario ${ }^{43}$. La giurisprudenza ha introdotto alcuni correttivi alla regola di piena libertà delle parti nella definizione delle unità di negoziazione: senza entrare nei dettagli, da una parte, esse andranno definite in modo razionale, appropriato e non discriminatorio, in vista di una regolazione unitaria delle condizioni di lavoro, per quanto attiene sia alle tipologie di attività economiche sia alla porzione di territorio; dall'altra, il campo di applicazione del contratto collettivo dovrà corrispondere ad un'area di legittimità (rappresentatività) delle organizzazioni stipulanti, atteso che queste ultime non potranno definire un'unità negoziale più ampia di quella che deriva dalla loro legittimazione ${ }^{44}$.

Altri casi riconducibili al tipo $b$ ) potrebbero, ad esempio, essere

tecipazione dei lavoratori): v. la decisione del 29 novembre 2019, n. 2019-816 (cfr. altresì la puntuale ricostruzione del commento reperibile al sito https://www.conseil-constitutionnel.fr/decision/2019/2019816QPC.htm, consultato l'ultima volta il 28 febbraio 2020).

42 Un cenno in F. SiotTo, La categoria come ambito di applicazione e perimetro di misurazione della rappresentanza, cit., p. 341 nota 96.

43 (Trad. mia). STC 136/1987, 22 luglio 1987. Per un breve confronto con il sistema adottato durante il franchismo, nel quale era la legge a fissare le unità di contrattazione: J.C. Villalon, Compendio de derecho del trabajo, cit., p. 546.

44 Cfr. J.R. Mercader Uguina, Art. 83. Unidades de negociación, in J.C. ViLLALon, et al. (dir.), Comentarios al estatuto de los trabajadores, IV ed., Madrid, Lex Nova-Thomson Reuters, 2016, p. 990 ss., P. Nieto Rojas, La respuesta de la negociacion colectiva sectorial a las empresas multiservicios, in Riv. Inf. Lab., 2017, 7, p. 229 ss., J. Gorelli Harnandez, La concurrencia entre convenios en el ordenamento español, in Variaz. Temi. Dir. Lav., 2018, p. 1129 ss. 
tratti dal diritto olandese $\mathrm{e}^{45} \mathrm{o}$ da quello tedesco ${ }^{46}$, nei quali i contratti collettivi stabiliscono il proprio campo di applicazione ed il potere esecutivo può disporne ex post l'obbligatorietà erga omnes ${ }^{47}$. Non occorre tuttavia proseguire oltre, atteso che dagli spunti forniti possono già trarsi alcune brevi considerazioni.

\subsection{Considerazioni conclusive}

Le soluzioni tecniche adottabili ai fini di una ripartizione del sistema contrattuale in unità negoziali sono molteplici. Tra di esse, si è illustrato come un criterio ordinatore possa essere rappresentato dalla fase nella quale interviene la definizione delle unità negoziali, se antecedente o contestuale rispetto allo svolgimento della contrattazione collettiva. Peraltro, tale criterio non è sovrapponibile alla più consueta ripartizione che opera, tra $\mathrm{i}$ sistemi di contrattazione collettiva ad efficacia erga omnes, in base al ricorso o meno a provvedimenti di estensione emanati dal potere esecutivo dopo la stipulazione dei contratti collettivi.

Infatti, tra i sistemi nei quali l'efficacia generale dei contratti collettivi è disposta con atto di estensione del potere esecutivo rientrano sia il Belgio (ove le unità negoziali sono fissate ex ante per decreto) sia la Francia (ove sono i contratti collettivi a stabilire il proprio campo di applicazione). Ne è una prova anche la Spagna: pur rientrando tra i sistemi nei quali l'ambito di applicazione dei contratti collettivi è determinato dalle parti stipulanti, la loro efficacia generale (si hanno in mente quelli cd. statutari) è prevista direttamente dalla legge, senza che occorra adottare provvedimenti di estensione ad hoc.

La distinzione che si è proposta è invece un buon test di veri-

45 Cfr. A. JacoBs, Labour law in the Netherlands, II ed., Alphen aan den Rijn, Kluwer law international, 2015, pp. 258 ss., 266 ss.

46 Cfr. T. Schulten, The role of extension in German collective bargaining, in S. Hayter, J. Visser (eds), Collective Agreements: Extending Labour Protection, cit., p. 71 ss.

47 Sulle recenti modifiche intervenute nel sistema tedesco in relazione alle procedure di estensione erga omnes degli effetti dei contratti collettivi cfr. C. CARTA, $L a$ crisi della contrattazione di settore in Germania, in Lav. Dir., 2019, p. 645 ss. 
fica per gli equilibri che, in caso di intervento della legge in questa materia, si possono stabilire tra eteronomia e autonomia nella definizione delle unità negoziali. Di primo acchito, parrebbe che $\mathrm{i}$ sistemi nei quali la definizione delle unità negoziali interviene in una fase precedente - per così dire, "a tavolino" - rispetto alla stipulazione dei contratti collettivi, siano anche quelli che implicano un intervento più intenso dei pubblici poteri e, perciò, una compressione più profonda dell'autonomia delle parti sociali. È pur vero, tuttavia, che l'esperienza belga sembra suggerire che, anche in un sistema di questo tipo, le prerogative sostanziali possono essere attribuite alle parti sociali, attraverso una sorta di diritto di iniziativa.

Lasciare alle organizzazioni stipulanti la definizione delle unità negoziali della contrattazione collettiva dovrebbe essere indice di una protezione ampia ed effettiva di quel particolare profilo dell'autonomia sindacale che consiste nella scelta dei modi più opportuni per intervenire nella realtà socioeconomica. Limitazioni più o meno intense dell'autonomia sindacale potrebbero comunque essere stabilite dalla legge, per evitare che tali scelte siano arbitrarie o irrazionali (è il caso spagnolo, per come risulta dall'interpretazione giurisprudenziale). Inoltre, le recenti vicende francesi dimostrano che, anche in un sistema basato sulla libertà delle parti stipulanti di definire le unità negoziali, non può escludersi che gli equilibri tra eteronomia e autonomia possano cambiare, da un certo momento in avanti, con le trasformazioni del sistema contrattuale e in base alle sensibilità del legislatore storico.

D'altro canto, da un punto di vista operativo i sistemi basati sulla definizione delle unità negoziali da parte dei contratti collettivi sono per forza di cose più esposti a rischi di sovrapposizione tra gli ambiti di applicazione dei contratti collettivi ${ }^{48}$. Di conseguenza, non è escluso che il controllo eteronomo, cacciato dalla porta, finisca per rientrare dalla finestra, per mezzo di una razionalizzazione degli agenti negoziali oppure mediante una selezione ex post dei contratti

48 Un cenno in tal senso da parte di J. Gorelli Harnandez, La concurrencia entre convenios en el ordenamento español, cit., p. 1139. 
collettivi applicabili ai rapporti di lavoro, operata da un'autorità amministrativa o dalla giurisprudenza.

Riepilogando, da una legge che estenda gli effetti dei contratti collettivi non può che derivare una compressione più o meno intensa della sfera di autonomia che le parti sociali serbano in questo ambito.

Tuttavia, l'esperienza comparata suggerisce che, nella materia che ci occupa, sono pensabili soluzioni equilibrate: $i$ brevi spunti di questo paragrafo hanno consentito di illustrare alcuni congegni che consentono di pervenire ad una definizione delle unità negoziali, non sempre ottimale (si pensi alle recenti tribolazioni del sistema francese), ma per lo più in grado di consentire il governo del sistema contrattuale. La riflessione non può che giovarsi da un esame, sia pure sommario, delle soluzioni adottate in altri ordinamenti, ma ben altro discorso è quello relativo alle normative che, nell'ordinamento italiano, potrebbero essere (nel caso in cui sia attuato l'art. 39 Cost.) o siano state effettivamente emanate (v. il d.lgs. n. 165/2001 e la l. n. 146/1990). Se è vero che il diavolo è nei dettagli, gioverà interrogarsi più a fondo su queste normative, che finora sono state soltanto evocate.

4. Rassegna delle soluzioni tecniche avanzate per la definizione delle unità negoziali nelle proposte di attuazione dell'art. 39 Cost.

Si prenderanno in esame alcune tra le più importanti proposte avanzate, dall'entrata in vigore della Costituzione in avanti, al fine di dare attuazione alla seconda parte dell'art. 39 Cost., per verificare quali soluzioni tecniche siano state ipotizzate per definire le unità negoziali della contrattazione collettiva.

\subsection{Dall'entrata in vigore della Costituzione ai primi anni Ses- santa}

Nei quattro anni che trascorsero tra l'abrogazione dell'ordinamento corporativo e l'entrata in vigore della Costituzione e, ancor 
più, dopo tale momento, furono discussi tra le forze politiche diversi progetti di legislazione sindacale ${ }^{49}$. Una delle difficoltà da fronteggiare consisteva nel come contemperare la libertà sindacale con $\mathrm{i}$ meccanismi di definizione delle unità negoziali dei contratti collettivi con effetti erga omnes.

Appariva chiaro che il congegno ideato dal costituente, giusta la progressione interna in vista del conseguimento dell'efficacia generale, richiedesse una determinazione ex ante delle unità di negoziazione $^{50}$. L'istituzione delle rappresentanze unitarie avrebbe imposto di stabilire in primis per quali unità costituirle. Ciò, peraltro, in modo compatibile con il pluralismo sindacale emerso dalla scissione della Cgil unitaria, atteso che in ogni categoria si sarebbero potute costituire più associazioni registrate, in vista di una contrattazione collettiva comunque unitaria: fin dal 1949 la voce autorevole di Santoro-Passarelli aveva ricondotto tale processo all'essenza dell'insegnamento «della dottrina sociale cristiana, espresso con la formula "sindacato libero nella professione organizzata" $\gg^{51}$. La delimitazione delle categorie avrebbe definito in anticipo e in modo oggettivo il campo della contesa negoziale tra le organizzazioni dei lavoratori e dei datori di lavoro.

L'operatività del congegno complessivo avrebbe richiesto una suddivisione in comparti delle forze produttive e del lavoro, ai fini sia della rilevazione del numero di iscritti alle associazioni sindacali (necessaria per l'attribuzione dei voti proporzionali), sia della distribuzione delle competenze negoziali tra le rappresentanze unitarie. L'ipotesi più discussa era quella dell'istituzione di un'anagrafe del

49 Cfr. M. Grand, I problemi del lavoro negli orientamenti della dottrina giuridica: organizzazione sindacale, contratto collettivo e sciopero, in S. ZANINELLI (a cura di), Il sindacato nuovo. Politica ed organizzazione del movimento sindacale in Italia negli anni 1943-1955, Milano, Franco Angeli, 1981, p. 185 ss.

50 Cfr. M. Persiani, I soggetti del contratto collettivo con efficacia generale, in Dir. Lav., 1958, I, p. 88 ss. Più di recente, L. Mariucci, L'attualità di un dibattito antico. Introduzione a L'attuazione degli articoli 39 e 46 della Costituzione. Tre proposte a confronto, cit., p. 12.

51 L'autore, in polemica con Carnelutti, espresse tale posizione nel corso del I Congresso nazionale dei giuristi cattolici, i cui atti furono pubblicati in Iustitia, 1949 (per la citazione p. 86). 
lavoro e/o delle imprese ${ }^{52}$, della cui legittimità in relazione al principio di libertà sindacale si sarebbe potuto discutere, ma che di certo avrebbe implicato una «pesante bardatura amministrativa di dubbia utilità e comunque di difficile attuazione» ${ }^{53}$.

Ci si riferisce, in particolare, al d.d.l. cd. Rubinacci (n. 2380), depositato alla Camera dei deputati il 4 dicembre 1951, nel corso della I legislatura ${ }^{54}$. Il progetto prevedeva l'istituzione di un'anagrafe per il censimento delle imprese in base al tipo di attività svolto ${ }^{55}$. Nello schema proposto, i meccanismi per la ripartizione in categorie del sistema sindacale avrebbero agito mediante l'anagrafe delle imprese ed i procedimenti di registrazione delle associazioni sindacali ${ }^{56}$. Lo si desumeva dall'art. 1 («Per ogni categoria professionale e per ogni circoscrizione territoriale possono essere costituite una o più associazioni sindacali») e dall'art. 3 («Le associazioni sindacali dei lavoratori, per ottenere la registrazione, debbono avere un numero di associati almeno pari al dieci per cento del numero medio dei lavoratori occupati nelle imprese del ramo di attività economica cui l'associazione si riferisce»), che anteponevano la definizione delle categorie alla registrazione delle associazioni sindacali. Tuttavia, dato che il progetto Rubinacci non arrivò all'esame dell'aula, i nodi relativi alla definizione delle unità contrattuali non furono sciolti.

Nel 1960, comunque, le Osservazioni e proposte sull'attuazione degli articoli 39 e 40 della Costituzione formulate dal Cnel operavano alcune importanti precisazioni, che tenevano conto della rilevanza che, in questa materia, assume il principio di libertà sindacale ${ }^{57}$. Secondo la considerazione n. 9 («Formazione delle rappresentanze unitarie»), la legge avrebbe dovuto rimettere alle associazioni sinda-

52 In forme che erano talvolta ricalcate sui meccanismi del diritto corporativo: v. ad esempio G. Maranini, A. Marzotto, L'art. 39 della Costituzione e l'organizzazione pubblica della categoria professionale, in Riv. Dir. Lav., 1949, I, p. 181 ss.

53 Sono parole di G. Pera, Problemi costituzionali del diritto sindacale italiano, cit., p. 60.

54 Vedilo in Riv. Giur. Lav., 1951, I, p. 263 ss. Per alcune considerazioni v. G. Pera, L'obbligatorietà dei contratti collettivi di lavoro, cit.

55 Cfr. G. Mazzoni, L'azione sindacale e lo "statuto dei lavoratori", cit., p. 102 ss. ma spec. p. 104.

56 Un cenno in A. LASSANDARI, Sulla verifica di rappresentatività delle organizzazioni sindacali datoriali, cit., pp. 9-10.

57 Vedile in G. SuppIeJ, Fonti per lo studio del diritto sindacale, cit., p. 163 ss. 
cali che promuovono la costituzione delle rappresentanze unitarie la prerogativa di indicare per quale categoria promuovere la contrattazione collettiva: «Dovrà cioè consentirsi di stipulare contratti collettivi con efficacia generale non solo per regolare i rapporti di lavoro dei dipendenti da aziende appartenenti ad uno stesso settore merceologico, od operanti nel medesimo territorio, ma anche per regolare uniformemente i rapporti caratterizzati da qualunque altro comune elemento oggettivamente accertabile, liberamente scelto dalle associazioni sindacali nell'esercizio della propria autonomia. In relazione a questi princìpi dovrà intendersi la nozione di categoria suscettibile di essere assoggettata ad una disciplina collettiva uniforme».

È noto che le vicende politico-sindacali dell'epoca impedirono che queste ed altre proposte fossero seriamente prese in considerazione $^{58}$. Le difficoltà tecniche si intrecciavano allora con quelle politiche e non si trattava «in realtà di due diversi ordini di difficoltà, ma di difficoltà politiche e tecniche allo stesso tempo, cioè di difficoltà di trovare soluzioni tecniche che non facciano sorgere problemi politici, a loro volta difficilmente risolubili» ${ }^{59}$. La memoria ancora fresca dell'esperienza corporativa - nella quale, ugualmente, la categoria precedeva il contratto collettivo -, spingeva, come noto, le associazioni sindacali a opporsi a ogni intrusione dei pubblici poteri nella sfera organizzativa e negoziale ${ }^{60}$. Si temeva per di più che simili congegni avrebbero ostacolato la crescita del sistema contrattuale, allorché alla metà degli anni Cinquanta se ne iniziava a presagire uno sviluppo cd. articolato (l'individuazione di nuove aree negoziali delineate sulla dimensione di impresa si sarebbe dovuta confrontare con un regime, se non di autorizzazioni, di passaggi formali) ${ }^{61}$.

58 Un passaggio in L. GAETA, «La terza dimensione del diritto»: legge e contratto collettivo nel Novecento italiano, in AA.VV., Legge e contrattazione collettiva nel diritto del lavoro post-statutario. Atti delle giornate di studio di diritto del lavoro Napoli, 16-17 giugno 2016, Milano, Giuffrè, 2017, pp. 32-35, ma passim.

59 Così, G. Suppiej, Fonti per lo studio del diritto sindacale, cit., p. 158.

60 M. NAPOLI, Il quadro giuridico-istituzionale, in G.P. CELla, T. TReu (a cura di), Le nuove relazioni industriali. L'esperienza italiana nella prospettiva europea, cit., pp. 54-55.

61 G. MAzzoni, I limiti di applicabilità dell'art. 2070, cit., p. 111. 
Questi elementi non andrebbero sottovalutati. Nella lettura successiva dell'art. 39 Cost. è rimasto avvinto il convincimento che la libertà di determinare gli ambiti di applicazione dei contratti collettivi finirebbe immancabilmente per essere sacrificata sull'altare di una legge che disponesse l'efficacia generale dei contratti collettivi, non soltanto oltre un limite accettabile per le parti sociali, ma altresì in modo incompatibile con il principio di libertà sindacale ${ }^{62}$ : da qui, il rilievo di un'insanabile contraddizione interna alla norma costituzionale, ossia la convinzione che non vi si possa dare attuazione senza al tempo stesso violare il principio di fondo ${ }^{63}$.

\subsection{Riprende il dibattito sulla legge sindacale: gli anni Ottanta e Novanta}

Una proposta di legislazione sul contratto collettivo fu discussa nuovamente nel 1986. Il quadro era mutato: il sistema contrattuale aveva raggiunto una copertura praticamente integrale del sistema produttivo, con l'eccezione (ovviamente) delle aree di lavoro nero e del sommerso e, per lo più, anche delle imprese di piccolissime dimensioni ${ }^{64}$. Un erga omnes senza le norme, di fatto ${ }^{65}$. Dopotutto sottolineava Suppiej - con la norma inattuata «ci siamo abituati a convivere ${ }^{66}$. Il problema dell'efficacia soggettiva del contratto collettivo si presentava nello scenario del governo del dissenso, da una parte, di organizzazioni sindacali contrarie alla conclusione di ac-

62 Cfr. per qualche spunto G. SAntoro-Passarelli, Sulla libertà sindacale dell'imprenditore, in Riv. Trim. Dir. Proc. Civ., 1976, pp. 176-178.

63 Fornisce, di recente, uno svolgimento di questa tesi, che risale alla prolusione bolognese di Mancini del 1962, P. Ichino, La crepa interna dell'art. 39 della Costituzione, cit.: «si torna a discutere dell'opportunità di dare attuazione alla seconda parte dell'art. 39 della Costituzione. Chi questo propone farà bene a riflettere sui motivi che hanno indotto gran parte della dottrina giussindacalistica a ritenere che questa norma costituzionale sia nata male. Di più: che il suo stesso impianto sia strutturalmente sbagliato».

64 R. Scognamiglio, A proposito di una recente iniziativa per la disciplina legislativa del contratto collettivo, in Mass. Giur. Lav., 1986, p. 684.

65 M. Rusciano, Introduzione. Che fare dell'art. 39 della Costituzione?, cit., p. 442.

66 G. SuppIEJ, La contrattazione collettiva tra crisi economica e riforme costituzionali, in Riv. It. Dir. Lav., 1986, I, p. 229. 
cordi non acquisitivi ${ }^{67}$ e, dall'altra, di lavoratori penalizzati da operazioni di gestione negoziata delle crisi d'impresa ${ }^{68}$.

Non per caso l'iniziativa fu presa dall'Unione industriali di Torino, che invitò Giuseppe Pera a predisporre un progetto di legge sul contratto collettivo. Il progetto, unitamente a un secondo che il celebre giurista elaborò di propria iniziativa, fu discusso nel corso di una giornata di studi tenutasi a Torino sul tema Per una disciplina legislativa del contratto collettivo ${ }^{69}$. L'accoglienza della dottrina fu nel complesso critica $^{70}$, ma qui interessa porre l'attenzione su alcuni aspetti soltanto dei due progetti.

Il punto n. 8) del primo progetto prevedeva che «L'accordo o il contratto collettivo debbono contenere la determinazione del tipo di imprese o di unità di lavoro di riferimento, dell'area territoriale di applicazione, dei rapporti di lavoro regolati secondo la ripartizione per categorie di cui all'art. 2095 c.c.». Il progetto attribuiva, quindi, la determinazione delle unità negoziali della contrattazione collettiva alle organizzazioni stipulanti, sia pure con la precisazione che ciò sarebbe dovuto avvenire dal punto di vista della tipologia delle imprese o delle unità di lavoro, del livello territoriale e delle categorie di lavoratori. Il punto n. 2) del secondo progetto si limitava a stabilire che sarebbero divenuti obbligatori «per tutti gli appartenenti alla categoria, in applicazione dell'art. 39 Cost.», i contratti collettivi stipulati sulla base di un criterio maggioritario, dal lato imprenditoriale, mediante una percentuale «delle imprese del tipo considerate» anche in riferimento all'entità del personale impiegato, dal lato dei lavoratori, del numero di iscritti in proporzione «al complesso dei lavoratori sindacalmente affiliati nella categoria interessata». Nel complesso, più che sui meccanismi di definizione delle categorie, l'attenzione era posta - comprensibilmente, dato il contesto nel quale erano maturate le due proposte - sull'adozione di un principio

67 L. MARIUCCI, Gli eterni ritorni: dentro, fuori o oltre l'art. 39 della Costituzione?, cit., p. 490.

68 G. GHezZI, L'intervento del giudice nel conflitto industriale, in Dir. Lav. Rel. Ind., 1983, p. 489 ss.

69 Vedi i testi delle proposte in Mass. Giur. Lav., 1986, pp. 683-684.

70 Lo ricorda R. Del Punta, Tre domande e una chiosa sulla legge sindacale, in F. CARINCI (a cura di), Legge o contrattazione? Una risposta sulla rappresentanza sindacale a Corte costituzionale n. 231/2013, cit., p. 163. 
maggioritario per la stipulazione dei contratti collettivi ${ }^{71}$. Era dato forse per acquisito il dato di fatto di una ripartizione del sistema contrattuale su unità negoziali prive di eccessive sovrapposizioni ${ }^{72}$.

L'unicità dei contratti collettivi nel proprio campo di applicazione cominciava, tuttavia, in quegli anni ad essere messa in discussione, con l'emergere dei movimenti sindacali extra-confederali, in alcuni segmenti della forza lavoro e nel lavoro organizzato (specialmente nei servizi e nel pubblico). Nelle proposte di attuazione dell'art. 39 Cost., si sarebbe, di lì in avanti, posto il problema di come garantire la coerenza nella struttura contrattuale senza, al contempo, pretermettere le istanze di gruppi professionali minoritari ${ }^{73}$.

Tre anni dopo lo svolgimento del convegno torinese, il tema tornava all'attenzione della comunità scientifica con i progetti cd. Giugni e Ghezzi, presentati, rispettivamente, nel gennaio e nel marzo 1989 al Senato della Repubblica e alla Camera dei deputati ${ }^{74}$. Entrambi, in linea con quanto proposto dalla Commissione bicamerale Bozzi, proponevano di modificare la seconda parte dell'art. 39 della Costituzione $^{75}$ : le disposizioni considerate più anacronistiche sarebbero state sostituite, in primo luogo, dal riferimento alla democraticità dell'organizzazione sindacale e, in secondo luogo, da un rinvio alla legge ordinaria per l'individuazione di criteri di rappresentatività che avrebbero consentito di stipulare contratti collettivi con efficacia generale (entrambi i progetti) e di conseguire ulteriori finalità (progetto Ghezzi) ${ }^{76}$.

Ai nostri fini, riveste interesse, in particolare, un aspetto della

71 Coerentemente con le inclinazioni "trentanovistiche" del redattore dei progetti: S. Sciarra, Contratto collettivo, in Dig. Comm., IV, 1989, p. 63 ss.

72 Cfr. M. Rusciano, In tema di disciplina del contratto collettivo di lavoro, in Mass. Giur. Lav., 1986, p. 426.

73 G. Santoro-Passarelli, Prospettive di riforma della rappresentanza sindacale nel lavoro privato, in Arg. Dir. Lav., 1999, p. 45.

74 Vedili in Riv. It. Dir. Lav., 1989, III, pp. 137 ss., 153 ss., per i progetti di revisione dell'art. 39 Cost., pp. 142 ss., 157 ss., per i progetti di legge ordinaria. Un cenno in L. GAETA, «La terza dimensione del diritto»: legge e contratto collettivo nel Novecento italiano, cit., pp. 55-56.

75 Per alcune osservazioni cfr. G. SupPIEJ, La contrattazione collettiva tra crisi economica e riforme costituzionali, cit., pp. 228-229.

76 Cfr. B. VenezIANI, Il sindacato dalla rappresentanza alla rappresentatività, in Dir. Lav. Rel. Ind., 1989, p. 408 ss. 
proposta Giugni. L'art. 6 del d.d.l. ordinaria (di completamento della riforma costituzionale) prevedeva una particolare tipologia di referendum, con il quale, sia nel settore privato sia nel pubblico, «lavoratori appartenenti a un gruppo professionale omogeneo» avrebbero potuto separarsi dall'unità contrattuale di riferimento, ricorrendo alcune condizioni: (a) richiesta espressa non oltre otto mesi prima della scadenza del contratto collettivo; $(b)$ richiesta proveniente da un'associazione sindacale costituita tra i lavoratori del gruppo professionale omogeneo; $(c)$ adesione alla richiesta da parte di almeno il 35\% dei lavoratori appartenenti al suddetto gruppo professionale. In caso approvazione da parte di una maggioranza dei 2/3, il gruppo professionale non sarebbe più stato «compreso, dopo la scadenza, nel campo di applicazione del contratto collettivo». Peraltro, in caso di esito positivo del referendum di separazione, sarebbe stato onere dell'associazione sindacale attivarsi per ottenere il riconoscimento della controparte ai fini della stipulazione di un contratto collettivo, come da prassi nel sistema sindacale di fatto.

La proposta di legge suggeriva un approccio originale al problema della frammentazione della rappresentanza sindacale e della struttura contrattuale, che, in quegli anni, iniziava ad emergere. L'esistenza di gruppi professionali autonomi dalle maggiori centrali aveva indotto un'intensificazione della conflittualità, ma anche la creazione di nuove aree contrattuali, in quei casi in cui i nuovi soggetti avessero dimostrato una compattezza tale da essere riconosciuti come controparte negoziale dai datori di lavoro. Nel nuovo contesto, il referendum di separazione mirava a governare i conflitti più gravi, mediante una procedura democratica di "uscita" dalle aree contrattuali. Il modo in cui era congegnato avrebbe, da un lato, scoraggiato il protrarsi di conflitti lunghi e, dall'altro, impedito la conclusione di più contratti collettivi con ambiti di applicazione sovrapposti.

Nel 1999 il d.d.l. Gasperoni ${ }^{77}$ avanzava una nuova proposta di legislazione sindacale. Il progetto, che interveniva a distanza di alcuni anni dalla modifica per via referendaria dell'art. 19 dello Statuto dei lavoratori, era volto soprattutto alla regolazione della rappresen-

77 Vedilo in Arg. Dir. Lav., 1999, p. 227 ss. 
tanza e della contrattazione collettiva in azienda. L'art. 10 prevedeva, tuttavia, anche l'efficacia generale dei contratti collettivi nazionali, sulla base di un modello affine a quello invalso nel settore pubblico. Si prevedeva, in particolare, una procedura di misurazione della rappresentatività delle organizzazioni sindacali (art. 10) e datoriali (art. 11), le quali avrebbero potuto stipulare i contratti collettivi sulla base di un principio maggioritario. Il progetto tralasciava il problema sostanziale della definizione dei perimetri negoziali (che nel settore pubblico era risolto con l'attribuzione all'ARAN della rappresentanza per la parte datoriale, con una razionalizzazione del pluralismo sindacale e, soprattutto, con la definizione di comparti di contrattazione collettiva, per «settori omogenei o affini», sulla base di accordi collettivi). Un'approvazione senza ulteriori modifiche del progetto da parte del Parlamento avrebbe, perciò, sollevato questioni di non facile soluzione, nei casi, che di lì in avanti si sarebbero fatti più frequenti, di sovrapposizione tra le aree contrattuali ${ }^{78}$.

\subsection{La Carta dei diritti della Cgil e le proposte della rivista DLM e del gruppo Freccia Rossa}

Negli ultimi anni la questione della legge sindacale è tornata d'attualità ${ }^{79}$. Le situazioni di pluralismo contrattuale hanno assunto un peso considerevole negli anni della recessione economica e della successiva, debole, ripresa, con il risultato di rendere più evidente la crisi, sin lì in qualche modo sotterranea, della tradizionale concezione del contratto collettivo di categoria come punto di riferimento univoco nel proprio perimetro negoziale ${ }^{80}$. È significativo, a tale proposito, che la gran parte delle proposte di legge elaborate negli

78 G. SANTORO-Passarelli, Prospettive di riforma della rappresentanza sindacale nel lavoro privato, cit., pp. 44-45. V. per qualche chiosa anche A. LASSANDARI, Il contratto collettivo aziendale e decentrato, cit., pp. 290-291.

79 Cfr. R. De Luca Tamajo, Incertezze e contraddizioni del Diritto sindacale italiano: è tempo di regolamentazione legislativa, in Riv. It. Dir. Lav., 2018, I, p. 273 ss.

80 E. Villa, Crisi della funzione anticoncorrenziale del contratto collettivo nazionale, in A. Lassandari, F. Martelloni, P. Tuldini, C. Zoli (a cura di), La contrattazione collettiva nello spazio economico globale, cit., p. 73 ss. 
ultimi anni prendano in considerazione il problema della definizione dei perimetri contrattuali ${ }^{81}$.

La soluzione avanzata in una proposta di legge depositata nel 2018 in Parlamento (iniziativa delle deputate Gribaudo e Cantone) ${ }^{82}$, attribuisce a un decreto del Ministro del lavoro il compito di «stabilire le categorie e le aree contrattuali» ${ }^{83}$. Per come la norma è formulata, il meccanismo sarebbe incompatibile con l'art. 39, c. 1, Cost., dato che implicherebbe un'espropriazione completa della prerogativa delle parti sociali di stabilire gli ambiti di applicazione dei contratti collettivi, senza che sia prefigurata una sia pur minima forma di iniziativa sindacale o di concertazione sociale.

La proposta di legge di iniziativa popolare promossa dalla Cgil («Carta dei diritti universali del lavoro. Nuovo statuto di tutte le lavoratrici e di tutti i lavoratori», presentata alla Camera il 29 settembre 2016) mira ad una regolazione organica del sistema sindacale ${ }^{84}$. Il punto che interessa riguarda la perimetrazione delle aree contrattuali. Il progetto risolve il problema (art. 33, c. 3) attribuendo ad un accordo interconfederale (ad efficacia generale), adottato a maggioranza qualificata del 60\%, il compito di definire le aree contrattuali.

Anche la proposta della Rivista Diritti lavori mercati su «Disciplina delle relazioni sindacali, della contrattazione collettiva e della partecipazione dei lavoratori» preconizza una regolazione complessiva del sistema sindacale ${ }^{85}$. L'art. 3, c. 4, stabilisce che gli accordi

81 Per un quadro del dibattito dottrinale cfr. F. CARINCI (a cura di), Legge o contrattazione? Una risposta sulla rappresentanza sindacale a Corte costituzionale n. 231/2013, cit., L. Zoppoli, A. Zoppoli, M. Delfino (a cura di), Una nuova Costituzione per il sistema di relazioni sindacali?, cit., AA.VV., L'attuazione degli articoli 39 e 46 della Costituzione. Tre proposte a confronto, cit.

82 La Proposta di legge n. 788 d'iniziativa delle deputate Gribaudo e Cantone presentata alla Camera dei Deputati il 26 giugno 2018 (Norme sull'accertamento della rappresentatività delle organizzazioni sindacali dei lavoratori e dei datori di lavoro privati).

83 Art. 4 della Proposta n. 788, cit.

84 Per un quadro d'insieme (delle proposte Cgil, Dir. Lav. Merc. e Freccia Rossa), V. Leccese, Contrattazione a efficacia generale e sistema contrattuale: le proposte di legge Cgil, "Diritti lavori mercati» $e$ «Freccia Rossa», in AA.VV., L'attuazione degli articoli 39 e 46 della Costituzione. Tre proposte a confronto, cit., p. 75 ss.

85 Vedila in L. Zoppoli, A. Zoppoli, M. Delfino (a cura di), Una nuova Costituzione per il sistema di relazioni sindacali?, cit., p. 539 ss. 
interconfederali, da adottare in base a un criterio maggioritario, «regolano e raccordano gli ambiti di applicazione dei contratti collettivi nazionali»; si prevede altresì che «Nel caso non si raggiunga l'accordo entro sei mesi dalla richiesta di avviare le trattative da parte di una delle confederazioni [...] le categorie contrattuali vengono definite in via transitoria da una Commissione di nove esperti indipendenti composta e nominata dal Presidente della Repubblica, nell'ambito di venti nominativi indicati dal presidente della Camera dei Deputati». La decisione della Commissione potrebbe «essere sostituita in ogni momento dagli accordi interconfederali» ${ }^{86}$.

Entrambe tali soluzioni ${ }^{87}$ avrebbero il pregio di garantire stabilità in merito alle precondizioni della contrattazione di settore, con un meccanismo che lascerebbe alle parti sociali la prerogativa essenziale di definire il campo di azione negoziale ${ }^{88}$. Solleva dubbi, nella proposta $D L M$, l'eventualità di un intervento eteronomo, anche se dovrebbe solo fungere da stimolo al raggiungimento degli accordi interconfederali sui perimetri. D'altro canto, non si può trascurare che entrambe le soluzioni priverebbero, in sostanza, le federazioni di categoria - firmatarie dei contratti di settore - della prerogativa di definire gli ambiti di applicazione dei contratti collettivi, spostando il baricentro della struttura contrattuale verso le confederazioni. Ciò non soltanto si porrebbe in discontinuità con l'esperienza invalsa nelle relazioni industriali italiane, ma comporterebbe anche il rischio di ingessare le dinamiche negoziali del settore privato ${ }^{89}$, che sono contraddistinte da un dinamismo non facilmente coordinabile

86 Si prevede altresì una norma transitoria in base alla quale (art. 3, co. 5) «Fino alla stipulazione degli accordi di cui al comma precedente, si fa riferimento a quanto previsto dagli ultimi contratti collettivi nazionali stipulati prima dell'entrata in vigore della presente legge».

87 Cfr. A. Lassandari, Tre proposte per una legge sindacale, in AA.VV., L'attuazione degli articoli 39 e 46 della Costituzione. Tre proposte a confronto, cit., p. 50 .

88 A. Zoppoli, Sulle ragioni, oggi, di una regolazione a tutto tondo (del potere sindacale e) della contrattazione collettiva (in particolare, sulla proposta di legge di «Diritti lavori mercati»), in AA.VV., L'attuazione degli articoli 39 e 46 della Costituzione. Tre proposte a confronto, cit., p. 152.

89 F. SCARPELLI, Le tensioni del sistema di relazioni industriali e la riforma della rappresentanza, in AA.VV., L'attuazione degli articoli 39 e 46 della Costituzione. Tre proposte a confronto, cit., p. 172. 
dall'alto da parte dei vertici confederali ${ }^{90}$. Si porrebbero, infine, problemi di compatibilità con l'art. 39, c. 1, Cost., ove non si attribuisse alcuna voce in capitolo nella determinazione dei perimetri a gruppi professionali non rappresentati dalle maggiori centrali confederali. $\mathrm{Al}$ proposito, si potrebbe semmai recuperare l'idea del referendum di separazione dall'area contrattuale avanzata dalla proposta Giugni del 1989.

La «Proposta di intervento legislativo in materia sindacale», avanzata dal gruppo di studiosi "Freccia Rossa" mente di questioni legate alla rappresentanza sindacale ed alla contrattazione collettiva in azienda. Tuttavia, è significativo che, in questo caso, la scelta di astenersi dal proporre regole sull'efficacia erga omnes dei contratti collettivi di settore derivi anche dai dubbi che sarebbero generati da una predeterminazione dei perimetri contrattuali: l'intento è di «non condizionare il libero sviluppo della dinamica delle relazioni industriali, che per loro natura possono vedere e spesso vedono un'evoluzione dei confini dei settori contrattuali in relazione al mutamento dei processi produttivi, alle vicende associative... $\nu^{92}$. La proposta prevede alcuni accorgimenti che dovrebbero scongiurare fenomeni di dumping nelle ipotesi di rinvio dalla legge alla contrattazione collettiva, ove il rischio di fughe strumentali dal sistema contrattuale è particolarmente avvertito ${ }^{93}$. Tali accorgimenti presuppongono che la definizione dei perimetri della contrattazione nazionale sia intervenuta ad opera delle organizzazioni che stipulano i contratti ${ }^{94}$. La definizione delle aree contrattuali sarebbe, in qualche modo, un risvolto dei meccanismi di misurazione della rappresentatività, da attivare sulla base degli ambiti di applicazione dei

90 G. Proia, Ostacoli ed incognite per l'intervento legislativo sulla rappresentanza sindacale, in L. Zoppoli, A. Zoppoli, M. Delfino (a cura di), Una nuova Costituzione per il sistema di relazioni sindacali?, cit., p. 510.

91 Vedila in Riv. It. Dir. Lav., 2015, III, p. 205 ss.

92 Così, le Linee guida di una proposta di "Legge sindacale”, in Riv. It. Dir. Lav., 2015, III, p. 207.

93 V. LECCESE, La contrattazione collettiva nazionale oggi: caratteri, metamorfosi e criticità. Temi per il dibattito, cit., pp. 113-114.

94 Una «soluzione leggera», ad avviso di F. SCARPELLI, Le tensioni del sistema di relazioni industriali e la riforma della rappresentanza, cit. p. 172. 
contratti di settore ${ }^{95}$. La soluzione sarebbe in linea con le più recenti dinamiche degli accordi interconfederali, che preconizzano una verifica della rappresentatività a partire dai perimetri dei $\mathrm{Ccnl}^{96}$.

5. La definizione dei comparti di contrattazione nel settore pubblico

Il tema della definizione dei comparti per la contrattazione collettiva del settore pubblico esorta ad approfondire ulteriormente $\mathrm{i}$ ragionamenti svolti in questa ricerca, in una duplice direzione.

Da una parte, giova interrogarsi sulle disposizioni del d.lgs. n. 165/2001 (e loro precedenti) che sono volte a favorire una delimitazione delle categorie contrattuali; disposizioni, invero, di un certo peso, nel quadro della contrattazione del settore, il cui regolare svolgimento dipende dall'esistenza di una struttura negoziale ordinata, non meno di quanto dipenda da un'efficace selezione dei soggetti legittimati a prendervi parte ${ }^{97}$. Poiché tali congegni limitano, in vari modi, l'autonomia delle parti stipulanti di individuare le unità negoziali, non potrà d'altra parte eludersi il problema della loro compatibilità con il principio di libertà sindacale. Per illustrare tali svolgimenti, è opportuno riprendere, sia pure per grandi li-

95 B. CARUSO, Per un intervento eteronomo sulla rappresentanza sindacale: se non ora quando!, cit., p. 470.

96 V., supra, cap. II, § 4.

97 Come noto, oltre che dai meccanismi che consentono una delimitazione dei comparti di contrattazione, il pluralismo sindacale è governato dalle disposizioni che operano una selezione degli agenti negoziali. Da una parte, in conseguenza dell'attribuzione all'Aran della rappresentanza legale a fini negoziali delle amministrazioni pubbliche, ma anche della costituzione di comitati di settore che fungono da raccordo, nei meccanismi di rappresentanza, tra l'Agenzia e le pubbliche amministrazioni interessate dalla contrattazione collettiva. Da un'altra parte, mediante la previsione, per la partecipazione ai tavoli negoziali e per la stipulazione dei contratti collettivi, di determinate soglie di rappresentatività e di maggioranza, basate sul criterio associativo e sul criterio elettorale (cd. modello misto), che operano come filtro per le organizzazioni sindacali dei lavoratori. Per una ricostruzione del tema L. Zoppoli, M. Delfino, La rappresentanza delle pubbliche amministrazioni, in G. Proia (a cura di), Organizzazione sindacale e contrattazione collettiva, cit., p. 1033 ss., C. LAZZARI, Rappresentanza e rappresentatività sindacale nel lavoro pubblico, ivi, p. 1081 ss. 
nee, i principali passaggi dell'evoluzione delle discipline legislative in materia.

\subsection{Evoluzione delle discipline legislative e della corrispondente attività negoziale}

La legge-quadro sul pubblico impiego (spec. art. 5, c. 2, 1. n. 93/1983) attribuiva il compito di determinare il numero e la composizione dei comparti di contrattazione a un d.p.r., da emanare a seguito di una delibera del Consiglio dei Ministri, adottata su proposta del Presidente del Consiglio, sulla base di accordi definiti tra questi e le confederazioni sindacali maggiormente rappresentative sul piano nazionale; era previsto che le Regioni fossero «sentite» e al Parlamento fosse data «previa comunicazione». $\mathrm{Al}$ riguardo, è condivisibile l'opinione all'epoca prevalente, secondo la quale permaneva un margine di discrezionalità al Consiglio dei Ministri (con un ruolo incisivo del Presidente) nella definizione dei comparti, nonostante l'accordo sindacale ${ }^{98}$.

Le determinazioni principali in merito alla struttura contrattuale erano state assunte con scelte autoritative del legislatore: la definizione dei comparti faceva solo in parte eccezione ${ }^{99}$. È significativo al riguardo che il negoziato fosse stato indirizzato dalle disposizioni relative alla composizione della delegazione trattante per la parte pubblica (artt. 6-10), la quale avrebbe condizionato sia l'individuazione del numero sia la definizione dei comparti ${ }^{100}$. Ciò non deve stupire: nel pubblico come nel privato, la struttura della contratta-

98 Non si spiegherebbe altrimenti la diversità nella formulazione delle disposizioni previste più in generale per la stipulazione degli accordi sindacali: l'art. 6 non prevedeva l'emanazione di provvedimenti governativi sulla base di accordi sindacali, bensì, a seguito del corretto esperimento della procedura, il loro recepimento, entro il termine di dieci giorni dalla pronuncia della Corte dei Conti, mediante emanazione di un d.p.r., previa delibera del C.d.M. Cfr. T. Treu, La contrattazione collettiva nel pubblico impiego: ambiti e struttura, in Dir. Lav. Rel. Ind., 1994, p. 34.

99 M. Barbieri, (M.G. Garofalo), La contrattazione collettiva, cit., p. 692.

100 L. Zoppoli, I comparti pubblici dopo la riforma del rapporto di lavoro, in Lav. Inf., 1993, 10, p. 6. V. altresì A. Orsi BatTaglini, Relazioni sindacali e fonti normative nella legge quadro sul pubblico impiego, cit., pp. 6-13. 
zione collettiva è anzitutto determinata dalle configurazioni assunte dalle rappresentanze datoriali.

L'art. 5 della legge-quadro precisava, comunque, che il numero dei comparti dovesse essere «limitato» (c. 1) e che la loro composizione avrebbe tenuto conto di un criterio direttivo, consistente nell'elemento oggettivo dell'omogeneità o affinità dei settori (c. 4). Si stabiliva, inoltre, che per ciascun comparto si potesse stipulare un solo accordo collettivo (c. 1). All'esito di un confronto negoziale protrattosi per anni e della stipulazione di due accordi collettivi, il d.p.r. 5 marzo 1986, n. 68 individuò otto comparti di contrattazione $^{101}$.

Il fatto che la definizione dei comparti di contrattazione collettiva fosse solo in parte rimessa all'autonomia delle parti negoziali non toglie che alle confederazioni sindacali fossero attribuite importanti prerogative $^{102}$. Le determinazioni delle confederazioni, benché di carattere preparatorio rispetto alle scelte dell'esecutivo, avrebbero influito, infatti, sul peso rappresentativo delle organizzazioni di categoria ${ }^{103}$, così come sulle dinamiche e sul merito stesso degli accordi che queste ultime avrebbero stipulato ${ }^{104}$.

Tali andamenti sarebbero diventati più evidenti con l'introduzione (dal 1997) dei criteri di misurazione della rappresentatività e del principio maggioritario per la stipulazione dei contratti collettivi, ma anche all'epoca era chiaro che avere comparti più vasti avrebbe significato meno organizzazioni di categoria in grado di esse-

101 Il d.p.r. n. 68/1986, emanato sulla base di due accordi sindacali (un primo del 21 dicembre 1984 e un secondo, integrativo, dell'11 febbraio 1986), individuò i seguenti otto comparti di contrattazione: 1) personale dipendenti dai Ministeri; 2) personale degli enti pubblici non economici; 3) personale delle regioni e degli enti pubblici non economici da esse dipendenti, dei comuni, delle province, delle comunità montane, loro consorzi ed associazioni; 4) personale delle aziende e delle amministrazioni dello Stato ad ordinamento autonomo; 5) personale del Servizio sanitario nazionale; 6) personale delle istituzioni e degli enti di ricerca e sperimentazione; 7) personale della scuola; 8) personale dell'università.

102 L. Fiorillo, Le fonti di disciplina del rapporto di lavoro pubblico, Padova, Cedam, 1990, p. 67.

103 A. Vallebona, Alchimie del legislatore e occhiali del giurista nella riforma della contrattazione collettiva con le pubbliche amministrazioni, in Riv. It. Dir. Lav., 1998, II, p. 54.

104 T. TREU, La contrattazione collettiva nel pubblico impiego: ambiti e struttura, cit., p. 38 . 
re rappresentative nelle diverse unità e, così, meno soggetti tra i quali cercare l'intesa al tavolo negoziale (e, se si vuole, meno temi e meno rivendicazioni sui quali cercare una convergenza). E viceversa: comparti più angusti avrebbero facilitato il sovraffollamento sindacale e reso più complesse le trattative ${ }^{105}$. La soluzione ideale non esiste, se è vero, come i più attenti hanno rimarcato, che l'aver cercato di escludere dal gioco sindacale - anche mediante la definizione delle unità negoziali - alcuni segmenti del lavoro pubblico ha favorito, in quegli anni, una proliferazione di soggetti extra-confederali e un corrispondente incremento della conflittualità. In altri termini, la ricerca a tutti i costi della stabilità non è stata perseguita senza pagare alcun prezzo.

Con disposizioni nella sostanza non lontane da quelle del 1983, l'art. 45, d.lgs. n. 29/1993 (l.d. n. 421/1992, nel quadro della cd. prima privatizzazione) ${ }^{106}$ attribuiva a un d.p.c.m. il compito di determinare i comparti di contrattazione. Come in precedenza, tale decreto sarebbe stato emanato sulla base di un accordo sindacale, che era affidato ai negoziati tra l'istituenda Agenzia per la rappresentanza negoziale delle pubbliche amministrazioni e le confederazioni sindacali maggiormente rappresentative sul piano nazionale.

La mancata riproposizione della disposizione (art. 5, c. 1, leggequadro) che imponeva la stipulazione di un solo accordo per comparto, non equivaleva al superamento del principio dell'unicità del contratto per unità negoziale; essa era semplicemente divenuta superflua, dopo l'unificazione della rappresentanza di parte datoriale in un unico soggetto pubblico ${ }^{107}$.

La Conferenza dei presidenti delle Regioni sarebbe stata «sentita», per gli aspetti di interesse regionale, stando alla formulazione originaria $^{108}$. Dopo un intervento della Corte costituzionale, che in-

105 M. RiccIardi, Riflessioni sull'entrata a regime del nuovo sistema di relazioni sindacali nel lavoro pubblico, in Dir. Rel. Ind., 2002, p. 615.

106 Cfr. F. CARInci, Contrattazione e contratto collettivo nell'impiego pubblico "privatizzato", in Lav. Pubb. Amm., 2013, p. 493 ss.

107 M. BARbieri, (M.G. Garofalo), La contrattazione collettiva, cit., pp. 701702.

108 Veniva meno la «previa comunicazione» al Parlamento, che del resto «non gode - osservava M. BARBieri, (M.G. GAROFAlo), La contrattazione collettiva, cit., p. 700 -, nell'attuale temperie politico-istituzionale, di grandi fortune» (certo non sfuggirà che siamo nell'epoca dell'avvicendamento tra I e II Repubblica, dei governi 
vestì una varietà di questioni in tema di rapporto con le Regioni ${ }^{109}$, ciò fu sostituito da una più incisiva «intesa» con la Conferenza dei Presidenti delle Regioni e delle Province autonome di Trento e Bolzano (su aspetti di interesse regionale) ${ }^{110}$.

La legge rinnovava gli aspetti pubblicistici della procedura per la definizione dei comparti, soprattutto nel confronto con la stipulazione degli accordi quadro e dei contratti collettivi nazionali di comparto. L'incisività dell'intervento eteronomo acquisiva persino maggiore risalto che in passato, atteso che con la riforma del 1993 veniva meno il passaggio del recepimento degli accordi sindacali in atti normativi, che a lungo - anche prima della legge-quadro - aveva caratterizzato la negoziazione del settore ${ }^{111}$. Nell'esperienza applicativa avrebbe, comunque, prevalso la continuità con la struttura contrattuale stabilita nel vigore della disciplina del 1983; gli accordi ed il successivo d.p.c.m. avrebbero, in sostanza, confermato la definizione di otto comparti di contrattazione ${ }^{112}$.

La normativa del 1993 (art. 46, c. 1) prevedeva che per ciascuno dei comparti si individuasse un'area separata per la dirigenza. Tale soluzione scaturiva dal compromesso raggiunto con le maggiori centrali ${ }^{113}$ e risultava anomala rispetto a quella di tipo oriz-

tecnici, del Parlamento delegittimato, del risanamento delle finanze pubbliche dissestate e via discorrendo).

109 C. cost. 30 luglio 1993, n. 359, in Giust. Civ., 1994, p. 17 ss., nota E. Gragnoli.

110 Art. 15, d.lgs. n. 470/1993 (che riscrisse l'art. 45, cit.).

111 Passaggio burocratico che aveva ispirato l'incisiva metafora dell'accordo collettivo che si suicida per rinascere nelle forme di un atto unilaterale e autoritativo (U. Romagnoli, La resistibile ascesa del contrattualismo nel pubblico impiego, in Dir Lav. Rel. Ind., 1980, p. 184).

112 Il d.p.c.m. n. 593/1993, emanato sulla base dell'accordo sindacale del 19 luglio 1993 tra il Ministro per la funzione pubblica, delegato dal Presidente del Consiglio, e le confederazioni sindacali maggiormente rappresentative sul piano nazionale, confermava la presenza di otto comparti di negoziazione, così suddivisi: A) personale dipendente dai Ministeri; B) personale degli enti pubblici non economici; C) personale delle regioni e delle autonomie locali; D) personale delle aziende e delle amministrazioni dello Stato ad ordinamento autonomo; E) personale del Servizio sanitario nazionale; F) personale delle istituzioni e degli enti di ricerca e sperimentazione; G) personale della scuola; $\mathrm{H}$ ) personale dell'università.

113 Che come noto avrebbero per legge partecipato ai tavoli negoziali della dirigenza, per «esprimere gli interessi generali dei dipendenti pubblici» (T. TREU, La contrattazione collettiva nel pubblico impiego: ambiti e struttura, cit., p. 73) ma 
zontale invalsa nelle relazioni sindacali del settore privato (almeno nell'industria $)^{114}$, oltre che di dubbia legittimità rispetto ai criteri della legge delega ${ }^{115}$. Forte di una autonomia consolidatasi nel decennio precedente e di una singolare capacità di pressione sindacale, la dirigenza del Servizio sanitario nazionale aveva ottenuto la previsione espressa di «una apposita area di contrattazione» (art. 46, c. $3)^{116}$.

È con la cd. seconda privatizzazione del 1997-1998 - osservava D'Antona - che una «diversa dislocazione della legge rispetto all'autonomia collettiva si ha anche con riguardo alla struttura contrattuale» ${ }^{117}$. L'art. 45, cit. veniva sostituito con una nuova formulazione dall'art. 1, d.lgs. n. 396/1997 (delega ex art. 11, cc. 4, 6, 1. n. 59/1997). Veniva introdotto (art. 45, c. 3, d.lgs. n. 29/1993) un principio che nella sostanza di lì in avanti rimarrà fermo (ora, art. 40, c. 2, d.lgs. n. 165/2001) e che consiste nell'affidare la definizione dei comparti della contrattazione collettiva nazionale ad appositi accordi tra l'Aran e le confederazioni rappresentative: una novità considerata più che opportuna dalla dottrina ${ }^{118}$. Ciò, benché restasse fermo il criterio oggettivo per il quale i comparti avrebbero dovuto comprendere settori omogenei o affini (verosimilmente, al fine di precluderne lo scorporo su linee orizzontali o per categorie professionali) ${ }^{119}$.

Si prevedeva la costituzione di Comitati di settore, quale momento di raccordo tra Aran e amministrazioni pubbliche, ai fini del-

di fatto come «necessari compagni di trattativa - o forse come controparti aggiunte», chiosa M. BARBIERI, (M.G. Garofalo), La contrattazione collettiva, cit., p. 707.

114 T. TREU, La contrattazione collettiva nel pubblico impiego: ambiti e struttura, cit., pp. 36-37.

115 L'art. 2, c. 1, lett. g), n. 5), 1. n. 421/1992 preconizzava la definizione di «una apposita, separata area di contrattazione per il personale dirigenziale non compreso nella lettera $e$ )»: cfr. M. BARBIERI, (M.G. GAROFAlo), La contrattazione collettiva, cit., p. 707.

116 V. già l'art. 15, 1. n. 502/1992.

117 M. D’Antona, Lavoro pubblico e diritto del lavoro: la seconda privatizzazione del pubblico impiego nelle «leggi Bassanini», in Lav. Pubb. Amm., 1998, p. 51.

118 M. Barbieri, (M.G. Garofalo), Contrattazione collettiva e lavoro pubblico: un modello per tutti?, in Lav. Pubb. Amm., 1998, p. 411.

119 P. Campanella, (M.T. CARINCI), L'attuazione della legge delega «Bassanini»: il d.lgs. $n$. 396/1997 in tema di contrattazione collettiva e rappresentanza sindacale nel pubblico impiego, in Lav. Pubb. Amm., 1998, p. 69. 
la rappresentanza negoziale. Ciò avrebbe orientato gli esiti del negoziato sui comparti ${ }^{120}$ : se ne trae conferma anche dalla previsione che attribuiva a un organismo di coordinamento tra i comitati di settore l'esercizio delle funzioni di indirizzo e delle altre competenze inerenti alla contrattazione svolta in materia di definizione dei comparti dall'Aran ${ }^{121}$. Vi è chi osserva che Comitati di settore e comparti di contrattazione altro non sono se non due «facce della stessa medaglia di cui il Comitato rappresenta il profilo soggettivo, il comparto l'ambito d'aggregazione dell'interesse collettivo delle pubbliche amministrazioni e, dunque, l'aspetto teleologico degli stessi Comitati di settore» ${ }^{122}$.

La riforma del 1997 confermava la presenza di aree separate per la dirigenza, con la significativa apertura alla contrattualizzazione della dirigenza generale, prevedendo un'area separata per la dirigenza del ruolo sanitario (art. 45, c. 3$)^{123}$. L'ultimo periodo della disposizione prevedeva che per le figure professionali in posizione di elevata responsabilità, con compiti di direzione o che comportano l'iscrizione ad albi oppure tecnico scientifici e di ricerca, fosse-

120 P. CAmpanella, (M.T. CARInCI), L'attuazione della legge delega «Bassanini»: il d.lgs. $n$. 396/1997 in tema di contrattazione collettiva e rappresentanza sindacale nel pubblico impiego, cit., p. 104.

121 Si tenga presente che la riforma del 1997 (art. 3, d.lgs. n. 396/1997, che sostituiva l'art. 46, d.lgs. n. 29/1993, con un nuovo testo) prevedeva che tale organismo di coordinamento fosse presieduto, per conto del Governo, dal Ministro per la funzione pubblica (c. 5); tale disposizione è transitata inalterata nel d.lgs. n. 165/2001 (art. 41, c. 6), per essere soltanto di recente modificata con la riforma Brunetta: il testo vigente dell'art. 41 , (ora) c. 5 prevede che le funzioni di indirizzo e le altre competenze siano esercitate collegialmente dai comitati di settore (v. infra nel testo). Ulteriore riprova di quanto si osserva nel testo, circa la corrispondenza quantomeno tendenziale tra struttura della rappresentanza di parte datoriale pubblica e struttura della contrattazione collettiva: è significativo che l'art. 11, c. 1, Ccnq 2 giugno 1998 di definizione dei comparti (1998-2001) preveda che, «fermi rimanendo i comparti di contrattazione definiti con il presente accordo», «immediatamente dopo la costituzione dei Comitati di settore» si stipulerà un successivo contratto con il quale «si individueranno ...».

122 M. D’ONGHIA, I soggetti della contrattazione collettiva di parte pubblica: Comitati di settore e ARAN, in F. CARINCI, S. MAINARDI (a cura di), La terza riforma del Lavoro Pubblico: commentario al D.Lgs. 27 ottobre 2009, n. 150, aggiornato al "Collegato lavoro", Milanofiori-Assago, Ipsoa, 2011, p. 374.

123 V. Talamo, La contrattazione collettiva della dirigenza pubblica, in Giorn. Dir. Amm., 2004, p. 674 ss. 
ro «stabilite discipline distinte nell'ambito dei contratti collettivi di comparto».

Le parti, cui era finalmente rimessa la definizione dei comparti, confermavano, in un primo momento, la suddivisione in otto comparti di contrattazione ${ }^{124}$, rinviando ad un contratto «da stipularsi immediatamente dopo la costituzione dei Comitati di settore» la soluzione di alcune questioni in sospeso ${ }^{125}$. Nel 2000, un nuovo Ccnq portava a dieci il numero dei comparti ${ }^{126}$. Nel 1998 un Aq individuava quattro aree di contrattazione della dirigenza ${ }^{127}$, che sarebbero diventate cinque nel $2000^{128}$.

124 Il Ccnq 2 giugno 1998, cit., sostitutivo dell'accordo del 19 luglio 1993 recepito nel d.p.c.m. n. 593/1993, individuava i seguenti comparti: A) personale dipendente dai Ministeri; B) personale degli enti pubblici non economici; C) personale delle regioni e delle autonomie locali; D) personale del Servizio sanitario nazionale; E) personale delle istituzioni e degli enti di ricerca e sperimentazione; F) personale della scuola; G) personale dell'università; $H$ ) personale delle aziende e delle amministrazioni dello Stato ad ordinamento autonomo.

125 In particolare: a) l'individuazione di «autonome aree di contrattazione collettiva della dirigenza»; $b$ ) «la collocazione - nell'ambito dei comparti - delle figure professionali per la relativa distinta disciplina»; $c$ ) «la collocazione contrattuale dei segretari comunali e provinciali nell'ambito del comparto Regioni-Autonomie locali»; nonché la definizione di un «comparto di appartenenza degli enti o delle agenzie di nuova istituzione».

126 Ccnq 9 agosto 2000 per la modifica del Cenq 2 giugno 1998 relativo alla definizione dei comparti di contrattazione; in particolare, erano aggiunti i comparti: I) del personale dipendente dalla Presidenza del Consiglio dei Ministri; L) del personale dipendente dalla agenzia delle entrate, delle dogane, del territorio e del demanio, denominate "agenzie fiscali". I comparti sarebbero stati «operativi a partire dal quadriennio 2002-2005» (art. 6 del Ccnq). Contestualmente era, da un lato, modificata la composizione del comparto A) Ministeri, dall'altro lato, ed in conseguenza, individuata la composizione dei comparti I) - L).

127 L'Aq 24 novembre 1998 per la definizione delle autonome aree di contrattazione della dirigenza (1998 - 2001) individuava le seguenti aree: I) comprendente i dirigenti dei seguenti comparti, ivi compresi quelli di livello dirigenziale generale, ove previsti dai relativi ordinamenti: ministeri; enti pubblici non economici; aziende ed amministrazioni dello Stato anche ad ordinamento autonomo; istituzioni ed enti di ricerca e sperimentazione; università; II) comprendente i dirigenti del comparto delle Regioni e delle Autonomie locali; III) comprendente la dirigenza dei ruoli professionale, tecnico, amministrativo e sanitario del servizio sanitario nazionale; IV) relativa alla dirigenza medica, comprendente medici, veterinari ed odontoiatri del servizio sanitario nazionale.

128 Il Ccnq 9 agosto 2000 per la modifica dell'accordo del 24 novembre 1998 modificava la composizione dell'Area I) per ricomprendervi «tutti i dirigenti in servizio presso i Ministeri, la Presidenza del Consiglio dei Ministri e le Agenzie fiscali» 
Tornando al quadro normativo: transitate nel d.lgs. n. 165/2001 (art. 40, c. 2), le disposizioni introdotte nel 1997-1998 avrebbero subito, negli anni successivi all'entrata in vigore del testo unico, alcuni aggiustamenti ${ }^{129}$, ma nel loro significato sostanziale sarebbero rimaste tali, fino alla riforma del 2009.

Nel primo decennio 2000 l'attività negoziale sulla definizione dei comparti ha manifestato una certa intensità. Se ne darà conto in maniera sommaria, segnalando gli andamenti sul numero dei comparti e delle aree separate, con qualche notazione a margine; resterà in ombra, per economia di esposizione, il tema della composizione dei comparti.

Il Ccnq 18 dicembre 2002 (quadriennio 2002-2005) ha stabilito in undici il numero dei comparti di contrattazione collettiva ${ }^{130}$. Un Aq del 23 settembre 2004 ha fissato a otto il numero delle aree contrattuali autonome per la dirigenza ${ }^{131}$. Il numero dei comparti di

e introduceva una Area V) come «autonoma area della dirigenza scolastica, costituita a decorrere dal $1^{\circ}$ settembre 2000» (collocata nel comparto scuola).

129 Rassegna per punti:

i) l'art. 7 , c. 4 , 1. n. $145 / 2002$ introduceva un nuovo periodo nell'art. 40, c. 2 , cit., giusta il quale «I professionisti degli enti pubblici, già appartenenti alla $\mathrm{X}$ qualifica funzionale, i ricercatori e i tecnologi degli enti di ricerca, compresi quelli dell'ENEA, costituiscono, senza alcun onere aggiuntivo di spesa a carico delle amministrazioni interessate, unitamente alla dirigenza, in separata sezione, un'area contrattuale autonoma, nel rispetto della distinzione di ruolo e funzioni.

ii) L'art. 14, c. 2, 1. n. 229/2003 modificava l'ultimo periodo dell'art. 40, c. 2 , cit., con la seguente formulazione: «Per le figure professionali che, in posizione di elevata responsabilità, svolgono compiti di direzione o che comportano iscrizione ad albi e per gli archeologi e gli storici dell'arte aventi il requisito di cui all'articolo 1, comma 3, della legge 7 luglio 1988, n. 254, nonché per gli archivisti di Stato, i bibliotecari e gli esperti di cui all'articolo 2, comma 1, della medesima legge, che, in posizione di elevata responsabilità, svolgono compiti tecnico scientifici e di ricerca, sono stabilite discipline distinte nell'ambito dei contratti collettivi di comparto».

iii) L'art. 1, c. 125, 1. n. 311/2004 modificava il terzo periodo dell'art. 40, c. 2, cit., sopprimendo il seguente riferimento: «i ricercatori e i tecnologi degli enti di ricerca, compresi quelli dell'ENEA».

130 A) personale delle Agenzie fiscali; B) personale delle aziende e delle amministrazioni dello Stato ad ordinamento autonomo; C) personale degli enti pubblici non economici; D) personale delle Istituzioni di alta formazione e specializzazione artistica e musicale; E) personale delle istituzioni e degli enti di ricerca e sperimentazione; F) personale dei Ministeri; G) personale della Presidenza del Consiglio dei Ministri; H) personale delle Regioni e delle Autonomie locali; I) personale del Servizio sanitario nazionale; $\mathrm{L}$ ) personale della scuola; $\mathrm{M}$ ) personale dell'università.

131 I) dirigenti del comparto dei Ministeri e delle Amministrazioni dello Stato ad ordinamento autonomo; II) dirigenti del comparto Regioni e Autonomie locali; 
contrattazione è portato a dieci con il Ccnq 11 giugno 2007 (quadriennio 2006-2009) ${ }^{132}$. Con alcuni aggiustamenti interni rispetto al precedente del 2004, un Aq del $1^{\circ}$ febbraio 2008 (quadriennio 2006-2009) ha confermato in otto il numero delle aree separate per la dirigenza ${ }^{133}$.

È significativo che con questi ultimi due accordi (Ccnq 11 giugno 2007 e Aq $1^{\circ}$ febbraio 2008) le parti abbiano anticipato alcuni svolgimenti che diverranno manifesti negli anni a seguire, in tema di razionalizzazione della struttura contrattuale e di riduzione del numero di comparti di contrattazione e di aree separate per la dirigenza ${ }^{134}$ : tema

III) dirigenti dei ruoli sanitario, professionale, tecnico, amministrativo del comparto del Servizio sanitario nazionale; IV) dirigenza medico-veterinaria, comprendente medici, veterinari ed odontoiatri del comparto del Servizio sanitario nazionale; V) dirigenti dei comparti Scuola e Istituzioni di alta formazione e specializzazione artistica e musicale; VI) dirigenti dei comparti Agenzie fiscali e Enti pubblici non economici; VII) dirigenti dei comparti Università e Istituzioni ed enti di ricerca e sperimentazione; VIII) dirigenti del comparto della Presidenza del Consiglio dei Ministri.

132 Viene meno il comparto sub B) personale delle aziende e delle amministrazioni dello Stato ad ordinamento autonomo, per effetto degli avvicendamenti relativi all'inquadramento del Corpo nazionale dei Vigili del fuoco e del personale dei Monopoli di Stato. I comparti di contrattazione risultano così articolati: $\mathrm{A}$ ) personale delle Agenzie fiscali; B) personale degli enti pubblici non economici; C) personale delle Istituzioni di alta formazione e specializzazione artistica e musicale; D) personale delle istituzioni e degli enti di ricerca e sperimentazione; E) personale dei Ministeri; F) personale della Presidenza del Consiglio dei Ministri; G) personale delle Regioni e delle Autonomie locali; $\mathrm{H}$ ) personale del Servizio sanitario nazionale; I) personale della scuola; L) personale dell'università.

133 I) dirigenti del comparto dei Ministeri, ivi compresi i dirigenti delle professionalità sanitarie del Ministero della Salute di cui all'art. 2 della l. n. 120/2007; II) dirigenti del comparto delle Regioni e delle Autonomie locali; III) dirigenti dei ruoli sanitario, professionale, tecnico, amministrativo del comparto del Servizio sanitario nazionale; IV) dirigenza medico-veterinaria, comprendente medici, veterinari ed odontoiatri del comparto del Servizio sanitario nazionale; V) dirigenti dei comparti Scuola e Istituzioni di alta formazione e specializzazione artistica e musicale; VI) dirigenti dei comparti Agenzie fiscali e Enti pubblici non economici, ivi compresi i professionisti del comparto Enti pubblici non economici, collocati in apposita separata sezione ai sensi dell'art. 40, c. 2, d.lgs. n. 165/2001; VII) dirigenti dei comparti Università e Istituzioni ed enti di ricerca e sperimentazione; VIII) dirigenti del comparto della Presidenza del Consiglio dei Ministri.

134 L'art. 13 del Ccnq del 2007 prevede che «Al fine di evitare l'aumento dei comparti di contrattazione, nei contratti collettivi nazionali di lavoro le parti, ferma rimanendo l'unicità dei comparti di riferimento, potranno valutare l'opportunità di una articolazione della normativa contrattuale per specifici settori e sezioni secondo 
che sarebbe stato ripreso, come noto, dall'Aq del 22 gennaio 2009 di «Riforma degli assetti contrattuali», concluso tra il Governo e le maggiori organizzazioni dei datori di lavoro e dei lavoratori (con la significativa eccezione della Cgil) ed il cui punto 19 prevede che «le parti convengono sull'obiettivo di semplificare e ridurre il numero dei contratti collettivi nazionali di lavoro nei diversi comparti» ${ }^{135}$.

La cd. riforma Brunetta del lavoro pubblico (d.lgs. n. 150/2009) ha compresso gli spazi di intervento della contrattazione collettiva, rispetto alle precedenti fasi della contrattualizzazione ${ }^{136}$. La tendenza è stata in parte corretta dalla riforma Madia del 2015-2017 ${ }^{137}$. Quel che interessa per i temi che ci occupano sono i risvolti sulle attività di definizione dei comparti di contrattazione collettiva.

le denominazioni adottate dai Ccnl». Con una dichiarazione congiunta (Aran, Cgil, Cisl, Uil, Cisal, Confsal, Cgu, Rdb Cub) si afferma che al fine di «snellire ed accelerare il complesso delle procedure di contrattazione pubblica, le parti sottolineano l'opportunità di una riconduzione degli Enti di cui all'art. 70 del decreto legislativo 30 marzo 2001, n. 165 all'interno dei comparti di contrattazione di cui all'art. 40 dello stesso decreto, e ritengono che le obiettive specificità di cui trattasi possano trovare la giusta tutela nel Ccnl di comparto e, in particolare, nelle sessioni di contrattazione integrativa». Una dichiarazione congiunta dell'Aq del 2008 (Aran, Cgil, Cisl, Uil, Cida, Cisal, Confedir, Confsal, Cosmed) prevedeva l'istituzione di una commissione bilaterale, con il compito di formulare «ipotesi di aggregazione e riduzione delle aree dirigenziali, sulla base di un'approfondita analisi degli elementi di omogeneità e di quelli di specificità attinenti alla funzione dirigenziali nelle pubbliche amministrazioni». La medesima dichiarazione congiunta riprendeva testualmente quanto stabilito dalle parti con il Cenq del 2007 in materia di cd. Enti ex art. 70.

135 La questione non è invece specificamente ripresa dall'Intesa del 30 aprile 2009 (anch'essa conclusa senza la firma della Cgil) «per l'applicazione dell'Accordo Quadro sulla riforma degli assetti contrattuali del 22 gennaio 2009 ai comparti contrattuali del settore pubblico». Si tenga presente che l'Intesa è successiva alla legge delega 4 marzo 2009, n. 15, con la quale, (v. infra) le previsioni in materia di riduzione del numero dei comparti di contrattazione saranno attuate d'autorità (art. 3, c. 2, lett. h), n. 4).

136 Senza pretese selettive, v. A. VIScomi, La contrattazione collettiva nazionale, in L. Zoppoli (a cura di), Ideologia e tecnica nella riforma del lavoro pubblico, Napoli, Editoriale Scientifica, 2009, p. 41 ss., spec. 59, A. Alaimo, La contrattazione collettiva nel settore pubblico tra vincoli, controlli e "blocchi": dalla "riforma Brunetta" alla "manovra finanziaria" 2010, in Lav. Pubb. Amm., 2010, p. 287 ss., U. CARABELl, La "riforma Brunetta": un breve quadro sistematico delle novità legislative e alcune considerazioni critiche, in WP CSDLE "Massimo D'Antona".IT, 101/2010, F. CARINCI, Contrattazione e contratto collettivo nell'impiego pubblico "privatizzato", cit., spec. p. 504 ss.

137 Cfr. A. Bellavista, Contrattazione collettiva e partecipazione sindacale nella riforma Madia, in Giur. It., 2018, p. 1005 ss. 
A questo proposito, le modifiche introdotte nel 2009 sono state di prim'ordine $^{138}$. La formulazione dell'art. 40, c. 2, cit. è stata riveduta dall'art. 54, d.lgs. n. 150/2009. È stabilito in quattro il numero massimo di comparti di contrattazione collettiva, cui corrispondono altrettante aree per la dirigenza (primo periodo), con la previsione, comunque, di un'«apposita area o sezione contrattuale di un'area dirigenziale» per la dirigenza del Servizio sanitario nazionale (secondo periodo). Le specifiche professionalità possono essere riconosciute, a differenza che in passato, mediante la costituzione di «apposite sezioni contrattuali» (terzo e ultimo periodo). La Presidenza del Consiglio dei Ministri è esclusa dall'accorpamento delle unità negoziali, in base all'art. 74, c. 3, d.lgs. n. 150/2009 ed al successivo d.p.c.m. n. 226/2010.

L'art. 40, cit. non dispone più che le parti debbano considerare il criterio oggettivo della omogeneità e affinità dei settori, nella composizione dei comparti ${ }^{139}$. La struttura della contrattazione, d'altro canto, è preconizzata dalle disposizioni sulla costituzione dei Comitati di settore (art. 41, cc. 2-3, d.lgs. n. 165/2001). Uno di essi opera nell'ambito della Conferenza delle Regioni, con competenze per «le regioni, i relativi enti dipendenti, e le amministrazioni del Servizio sanitario nazionale» (c. 2) ${ }^{140}$. Un secondo, nell'ambito dell'Anci, dell'Upi e di Unioncamere, con competenze per enti locali, camere di commercio e segretari comunali e provinciali (c. 2). Il Presidente del Consiglio dei Ministri, tramite il Ministro per la pubblica amministrazione e l'innovazione, di concerto con il Ministro dell'Economia e delle Finanze, opera come Comitato di settore «Per tutte le altre amministrazioni» $(\text { c. } 3)^{141}$.

138 Cfr. R. SOLOPERTO, La contrattazione collettiva nazionale e integrativa (artt. 36 e 53-56), in M. Tiraboschi, F. Verbaro (a cura di), La nuova riforma del lavoro pubblico. Commento alla legge 4 marzo 2009, $n$. 15, e al decreto legislativo 27 ottobre 2009, n. 150, Milano, Giuffrè, 2010, p. 365 ss.

139 Il criterio oggettivo, a quanto consta, solo sporadicamente era stato preso in considerazione dalla giurisprudenza (v. T. Roma 31 gennaio 2001, in Rass. Avv. St., 2001, II, p. 199 ss., Cass. 27 novembre 2018, n. 30685, in Pluris per un obiter dictum, ma amplius G. Rucco, Commentario al d.lgs. 27 ottobre 2009, n. 150, Roma, SSPAL, 2010, p. 13).

140 La disposizione prevede che a tale Comitato di settore prenda parte un rappresentante del Governo, designato dal Ministro del lavoro, della salute e delle politiche sociali per le competenze delle amministrazioni del SSN.

141 È previsto che, «al fine di assicurare la salvaguardia delle specificità delle diverse amministrazioni e delle categorie di personale ivi comprese, gli indirizzi 
Non si prevede una corrispondenza necessaria tra struttura dei Comitati di settore e definizione dei comparti di contrattazione ${ }^{142}$, ma che i secondi, anche se non in modo pedissequo, debbano riprendere la struttura dei primi è conclusione che «da un punto di vista funzionale o operativo non appare certamente semplice da disattendere» ${ }^{143}$. La legge (spec. art. 66, d.lgs. n. 150/2009) risolve la questione dell'inquadramento contrattuale dei cd. Enti ex art. 70 (Enac, Asi, Cnipa, Unioncamere e Cnel) disponendone la collocazione nei comparti e nelle aree di contrattazione ex art. 40.

Tali previsioni sono rimaste lettera morta per alcuni anni, in corrispondenza del "blocco" della contrattazione collettiva nel settore pubblico, intervenuto tra il 2010 e il 2015 per ragioni di spesa pubblica $^{144}$. Una volta ristabilite le premesse giuridiche per lo svolgimento dei processi negoziali ${ }^{145}$, in vista, ormai, delle provviste finanziarie dei rinnovi, Aran e parti sociali hanno stipulato un Ccnq sulle unità di contrattazione collettiva (13 luglio 2016 per il triennio 2016-2018). L'accordo delinea quattro comparti di contrattazione (cui corrispondono 4 aree dirigenziali): A) Funzioni centrali; B) Funzioni locali; C) Istruzione e ricerca; D) Sanità. L'accordo ha aperto la via ai rinnovi dei contratti collettivi nazionali di comparto ${ }^{146}$ e delle aree previste dall'art. $7^{147}$.

sono emanati per il sistema scolastico, sentito il Ministro dell'istruzione, dell'università e della ricerca, nonché, per i rispettivi ambiti di competenza, sentiti i direttori delle Agenzie fiscali, la Conferenza dei rettori delle università italiane, le istanze rappresentative promosse dai presidenti degli enti di ricerca e degli enti pubblici non economici ed il presidente del Consiglio nazionale dell'economia e del lavoro».

142 A. VIscomi, La contrattazione collettiva nazionale, cit., pp. 55-56.

143 Così, C. Zoli, La struttura della contrattazione collettiva nel lavoro pubblico, cit., p. 167.

144 F. Guarriello, Crisi economica, contrattazione collettiva e ruolo della legge, in Dir. Lav. Rel. Ind., 2016, p. 21 ss.

145 C. cost. n. 178/2015, cit.

146 Per alcune notazioni, C. Russo, Aspettando Godot? Nuovi comparti e aree dirigenziali in attesa del rinnovo 2016-2018, in Dir. Lav. Merc., 2016, p. 439 ss., V. Talamo, Antefatti, luci ed ombre dei rinnovi contrattuali del pubblico impiego, in Giorn. Dir. Amm., 2018, p. 277 ss.

147 Ccnl dell'Area Istruzione e Ricerca (8 luglio 2019), Ccnl dell'Area Funzioni Centrali ( 9 ottobre 2019), Ccnl dell'Area Sanità (19 dicembre 2019). 
5.2. La limitata "sovranità" delle organizzazioni sindacali nella definizione dei comparti

Sia pure arrestandosi, invero, alla superficie delle questioni, la ricostruzione svolta ha consentito di afferrare la complessità dei meccanismi di definizione delle aree negoziali, in un contesto disciplinato dalla legge in modo minuzioso.

Fin dalla riforma del 1997-1998, la definizione dei comparti di contrattazione collettiva nazionale è rimessa alle previsioni adottate con accordi quadro stipulati periodicamente tra l'Aran e le confederazioni rappresentative, in vista dello svolgimento delle tornate contrattuali. In dottrina, questo «momento delicatissimo» per l'autonomia collettiva è associato alla "sovranità" delle parti negoziali ${ }^{148}$. In effetti, l'attribuzione alle parti della prerogativa di definire le unità negoziali è un principio cardine dell'intera contrattazione collettiva - come questa ricerca ha cercato, fin dai suoi esordi, di dimostrare che nel settore pubblico si è affermato relativamente di recente (ma con tempi di gestazione neanche troppo lunghi) ${ }^{149}$.

È indubbio che in materia di definizione dei comparti la riforma del 2009 abbia ridotto gli spazi riservati all'autonomia sindacale ${ }^{150}$. Anche da questo punto di vista le divergenze tra settore pubblico e privato continuano ad aumentare ${ }^{151}$. Peraltro, è discusso se un simile approccio sia adeguato alle sfide che la contrattazione collettiva del lavoro pubblico ha davanti a sé152.

148 M. Barbieri, (M.G. Garofalo), Contrattazione collettiva e lavoro pubblico: un modello per tutti?, cit., p. 411.

149 C. Zoli, La struttura della contrattazione collettiva nel lavoro pubblico, cit., p. 165.

150 R. Salomone, La procedura per la contrattazione, in G. Proia (a cura di), Organizzazione sindacale e contrattazione collettiva, cit., p. 1288.

151 G. NAtUllo, La nuova contrattazione collettiva nel lavoro pubblico: ambito e limiti, in Ist. Fed., 2009, p. 685 ss., A. Alaimo, La contrattazione collettiva nel settore pubblico tra vincoli, controlli e "blocchi", cit., p. 290.

152 A. VIscomi, La contrattazione collettiva nazionale, cit., p. 59. V. però, sulle motivazioni che potrebbero aver spinto il legislatore ad attivarsi per una forzosa riconduzione degli ambiti di negoziazione entro limiti predefiniti, V. TALAMO, La riforma del sistema di relazioni sindacali nel lavoro pubblico, in Giorn. Dir. Amm., 2010, p. 16 (riduzione dell'aggravio organizzativo dell'Aran e controllo della spesa derivante da una razionalizzazione dei trattamenti tra i diversi comparti). Concorde, C. Zoli, La struttura della contrattazione collettiva nel lavoro pubblico, cit., pp. 
Eppure, in tema, da questo piano di critica dell'opportunità, non si possono trarre conclusioni sicure sul piano della compatibilità con il principio di libertà sindacale. Si allude a un'opinione sostenuta, non senza argomenti, da larghi settori della dottrina, circa la violazione dell'art. 39, c. 1 , Cost. da parte dell'art. 40, c. 2, d.lgs. n. 165/2001 (ovviamente, nuova formulazione) ${ }^{153}$. In particolare, si sostiene che le disposizioni sul numero massimo di comparti di contrattazione e di aree separate, specialmente se lette alla luce di quelle sulla struttura dei Comitati di settore, comprimerebbero oltremisura la libertà di determinazione delle unità negoziali, in contrasto con l'art. 39, c. 1, Cost.

Tale orientamento si espone tuttavia ad alcune critiche sostanziali. In primo luogo, non si dovrebbe trascurare che la riduzione del numero di comparti (rispetto a quello stabilito dagli accordi allora vigenti), benché prevista d'imperio dalla legge, era stata anticipata da una serie di aperture delle parti sociali. Si hanno in mente, più che l'Aq separato del 22 gennaio 2009 (concluso senza la Cgil), il Ccnq 11 giugno 2007 e l'Aq $1^{\circ}$ febbraio 2008. Né può essere privo di significato che il Ccnq del 13 luglio 2016 abbia recepito e sviluppato le previsioni legislative sulla definizione dei comparti.

In secondo luogo, si dovrebbe tenere presente che le disposizioni citate sui Comitati di settore soltanto di fatto hanno una ricaduta sulla definizione dei comparti - e lo hanno secondo andamenti che, grosso modo, sono comuni ad ogni sistema di relazioni industriali, in cui la struttura contrattuale è tendenzialmente ricalcata sulle configurazioni dei processi produttivi e degli organismi di rappresen-

168-169, che amplia il ragionamento anche con riferimento agli obiettivi in materia di miglioramento della performance dei lavoratori pubblici.

153 Cfr. senza pretese di esaustività G. NAtullo, La nuova contrattazione collettiva nel lavoro pubblico: ambito e limiti, cit., p. 689 (un «simulacro del principio di autodeterminazione delle "categorie" contrattuali»), U. CARABELLI, La "riforma Brunetta": un breve quadro sistematico delle novità legislative e alcune considerazioni critiche, cit., p. 12, C. Zol, La struttura della contrattazione collettiva nel lavoro pubblico, cit., p. 169, M. Esposito, Il contratto collettivo del lavoro pubblico: così speciale, così reale, in L. Zoppoli, A. Zoppol, M. Delfino (a cura di), Una nuova Costituzione per il sistema di relazioni sindacali?, cit., p. 87, R. SALOMONE, La procedura per la contrattazione, cit., p. 1288 (nei termini dubitativi di uno spazio «assai meno "libero" di quanto non fosse in passato»). 
tanza datoriale ${ }^{154}$. Tali disposizioni possono essere considerate alla stregua di indicazioni, sia pure stringenti, con le quali la parte pubblica manifesta la propria disponibilità a negoziare, in certi ambiti, sulle materie oggetto di contrattazione collettiva: ossia, regole con le quali la parte pubblica organizza le proprie strutture in vista della negoziazione ${ }^{155}$.

Peraltro, è significativo che il Ccnq del 13 luglio 2016 sulla definizione dei comparti e delle aree separate abbia adottato, in concreto, scelte in parte diverse da quelle che erano state preconizzate con la riforma del 2009 (si pensi all'unificazione del comparto delle Funzioni locali, nonostante la duplicità dei Comitati di settore delle Regioni e degli Enti locali). Da questo punto di vista, non sembra si sia ingannato chi ha osservato che nonostante «l'indirizzo "centralista” della riforma», la previsione di un numero massimo di comparti «non sembra esporsi a dubbi di costituzionalità», atteso che «è confermata la competenza della contrattazione collettiva per la determinazione delle aree negoziali» ${ }^{156}$.

Invero, non è condivisibile l'affermazione secondo cui la riforma del 2009 abbia compresso oltre misura spazi di autonomia negoziale che prima erano riconosciuti dalla legge (nella definizione dei comparti di contrattazione). Occorre riconoscere come in questa materia il ruolo delle parti sociali sia sempre stato rilevante, ma al contempo ben circoscritto. La legge non soltanto stabilisce i criteri per prender parte alla negoziazione sui comparti ${ }^{157}$ e la procep. 227.

154 G.P. Cella, Quali cambiamenti per le relazioni industriali italiane?, cit.,

155 Cfr. M. Dell’Olio, Legge e contratto collettivo: autorità, funzione, libertà, in AA.VV., Le trasformazioni dei rapporti di lavoro pubblico e il sistema delle fonti: atti delle Giornate di studio di diritto del lavoro: L'Aquila, 31 maggio-1 giugno 1996, Milano, Giuffrè, 1997, p. 251 ss., P. CHieco, La natura organizzatoria delle norme di contrattualizzazione dei rapporti di lavoro alle dipendenze della p.a.: brevi spunti sistematici, ivi, p. 299 ss.; v. altresì G. PERA, La contrattazione collettiva a dominio confederale per il pubblico impiego, in Riv. It. Dir. Lav., 1998, II, p. 39 ss.

156 R. PEssi, Ripensando alla riforma della pubblica amministrazione: manutenzione o restaurazione?, in Mass. Giur. Lav., 2009, p. 297.

157 Art. 43, c. 4, d.lgs. n. 165/2001: «L'ARAN ammette alla contrattazione collettiva per la stipulazione degli accordi o contratti collettivi che definiscono o modificano i comparti o le aree o che regolano istituti comuni a tutte le pubbliche amministrazioni o riguardanti più comparti, le confederazioni sindacali alle quali, 
dura per stipulare i relativi accordi ${ }^{158}$, ma ha anche previsto (fino al 2009) un criterio oggettivo di omogeneità ed affinità per la loro definizione, oltre ad averne condizionato in più occasioni la stessa composizione professionale ${ }^{159}$, per non rimarcare che alcuni elementi di contesto.

In breve, o si afferma che l'intero impianto normativo sulla definizione dei comparti di contrattazione è incostituzionale, in base ad una lettura dell'art. 39, c. 1, Cost. per la quale non sarebbero ammissibili intromissioni di alcun tipo in tale sfera dell'autonomia sindacale ${ }^{160}$ (ma, in tal caso, la distinzione prima/dopo riforma del 2009 perderebbe in buona parte di significato), oppure si dovrebbe riconoscere che la riduzione a quattro del numero di comparti si iscrive, in fondo, in un quadro in cui l'autonomia sindacale si muove entro limiti angusti, ma è pur sempre esistente.

La questione si sposta, così, su un altro piano: quello della più generale compatibilità dei congegni descritti in questi paragrafi con il principio di libertà sindacale. Al proposito, sembra che sia opportuno leggere, per quanto possibile, con ragionevolezza, le limitazioni che ben difficilmente i poteri pubblici rinuncerebbero a porre in

in almeno due comparti o due aree contrattuali, siano affiliate organizzazioni sindacali rappresentative ai sensi del comma 1 ».

158 Art. 41, c. 5, d.lgs. n. 165/2001: «Per la stipulazione degli accordi che definiscono o modificano i comparti o le aree di contrattazione collettiva di cui all'articolo 40 , comma 2, o che regolano istituti comuni a più comparti o che si applicano a un comparto per il quale operano più comitati di settore le funzioni di indirizzo e le altre competenze inerenti alla contrattazione collettiva sono esercitate collegialmente dai comitati di settore». La procedura da osservare per la conclusione degli accordi è quella delineata dall'art. 47.

159 Basti pensare al caso della collocazione contrattuale di ricercatori e tecnologi degli enti pubblici di ricerca, che mutò in conseguenza di una disposizione collocata nella legge finanziaria per il 2005 (1. n. 311/2004), approvata dal Parlamento con voto di fiducia, che soppresse (v. supra) le parole «i ricercatori e i tecnologi degli enti di ricerca, compresi quelli dell'ENEA» dal terzo periodo dell'art. 40, c. 2, d.lgs. n. 165/2001: le parti negoziali dei contratti quadro ne presero atto con l'Aq 3 ottobre 2005 di modifica dell'Aq 23 settembre 2004 relativo alla definizione delle autonome aree di contrattazione della dirigenza per il quadriennio 20042005. In particolare, l'art. 1 (e unico) dell'accordo soppresse le corrispondenti parole dall'art. 3, c. 1, Aq 23 settembre 2004 (con dichiarazione a verbale critica di Confedir).

160 V. spec. le riflessioni di M. BARBIERI, Problemi costituzionali della contrattazione collettiva nel lavoro pubblico, cit., p. 419 ss. 
una materia come questa, atteso il rischio, diversamente, di abdicare all'esigenza di dotarsi di una struttura negoziale ordinata ${ }^{161}$. Il «cuore della questione» ${ }^{162}$ risiede probabilmente nel riconoscere come la "originaria" libertà di definizione delle unità negoziali debba, in questo settore, trovare un necessario contemperamento con i principi costituzionali di buon andamento e di imparzialità della pubblica amministrazione ${ }^{163}$. Ciò come espressione di un equilibrio con la fonte legale che rappresenta l'aspetto saliente della contrattazione collettiva del settore pubblico e che la Corte costituzionale ha nella sostanza ritenuto conforme alla carta fondamentale con la storica sentenza n. 309/1997 ${ }^{164}$.

6. Sciopero nei servizi pubblici essenziali e prestazioni indispensabili

Anche la disciplina in materia di sciopero nei servizi pubblici essenziali può fornire alcuni spunti preziosi per i ragionamenti svolti in questo capitolo.

La definizione del campo di applicazione degli accordi previsti dalla legge n. 146/1990 ai fini del contemperamento tra diritto di sciopero e diritti della persona costituzionalmente tutelati non è tra le questioni abitualmente affrontate dalla dottrina, al pari, ad esempio, di temi quali la definizione dei servizi essenziali o di quelli strumentali e/o accessori, la natura degli accordi collettivi e la loro effi-

161 S. MaInARDI, Fonti, poteri e responsabilità nella valutazione del merito dei dipendenti pubblici, in Lav. Pubb. Amm., 2009, p. 738.

162 Così, F. Scarpeldi, Le fonti e il contenuto del principio di libertà sindacale, cit., p. 76.

163 V. Speziale, Contratto collettivo e lavoro pubblico: rapporti tra le fonti e principi costituzionali, in Dir. Lav. Rel. Ind., 1996, p. 347. Più di recente, A. VISCoMI, Soggettività sindacale e categorie contrattuali, cit., p. 84.

164 C. cost. 16 ottobre 1997, n. 309, in Riv. It. Dir. Lav., 1998, II, p. 33 nota G. Pera, A. Vallebona, Lav. Pubb. Amm., 1998, p. 227, nota M. Barbieri, la quale, come noto, non ha tuttavia specificamente affrontato la questione della definizione dei comparti di contrattazione collettiva, come ha puntualizzato anche di recente R. Salomone, Rappresentatività sindacale, ambiti negoziali e procedure nella contrattazione collettiva del pubblico impiego, cit., p. 506 nota 34. 
cacia, le prerogative della Commissione di garanzia, la rappresentatività dei soggetti stipulanti, la titolarità del diritto di sciopero e così via $^{165}$. Ciò si spiega molto probabilmente con il fatto che la questione che ci occupa è risolta dagli stessi meccanismi applicativi della 1. n. 146/1990. Giova precisare che il ragionamento si concentrerà sulla definizione del campo di applicazione degli accordi collettivi di settore conclusi per le aziende erogatrici di servizi essenziali che operano nel privato, allo scopo di mettere in risalto alcune peculiarità rispetto al discorso svolto nei capp. I-II della ricerca sulla definizione delle categorie contrattuali; si tralasceranno, invece, gli accordi confinati al settore pubblico, dato che vi opera la disciplina speciale del testo unico del 2001, e gli accordi collettivi di livello aziendale, che sono applicati allo stesso soggetto datoriale stipulante e, perciò, non destano particolare interesse per il tema che ci occupa.

Una riflessione sulla definizione del campo di applicazione degli accordi collettivi conclusi ai sensi della 1. n. 146/1990 è sollecitata da due ordini di considerazioni. Da una parte, viene in rilievo la presenza della Commissione di garanzia, che è in grado di esercitare, nel confronto con le parti sociali, una serie di rilevanti prerogative, ai fini del contemperamento tra diritto di sciopero e godimento dei diritti della persona costituzionalmente tutelati. Dall'altra, la circostanza che, tra i diversi comparti dell'economia, proprio nel settore dei servizi si registrano le manifestazioni più intense di quel

165 La letteratura sul tema è amplissima. Si darà conto via via di alcuni contributi più attinenti al tema trattato, ma v. sin d'ora, senza pretese di esaustività, $\mathrm{P}$. Curzio, Autonomia collettiva e sciopero nei servizi essenziali, Bari, Cacucci, 1992, U. Romagnoli, M.V. Ballestrero, Art. 40 Supplemento: Legge 12 giugno 1990 n. 146. Norme sull'esercizio del diritto di sciopero nei servizi pubblici essenziali, in Giu. Branca (a cura di), Commentario della Costituzione, Bologna-Roma, Zanichelli-Società editrice del Foro italiano, 1994, P. PASCUCCI, Tecniche regolative dello sciopero nei servizi essenziali, Torino, Giappichelli, 1999, M. D’Onghia, M. RicCI (a cura di), Lo sciopero nei servizi pubblici essenziali, Milano, Giuffrè, 2003, E. Gragnol, Sciopero nei servizi pubblici essenziali, in Dig. Comm., Agg., 2003, p. 768 ss., A. Zoppoli, La titolarità sindacale del diritto di sciopero, Napoli, Jovene, 2006, M. Magnani (a cura di), Diritto di sciopero e assetto costituzionale, Milano, Giuffrè, 2010, F. LunARdon (a cura di), Conflitto, concertazione e partecipazione, P. II, Lo sciopero nei servizi pubblici essenziali, in F. CARInCI, M. Persiani, (dir. da), Trattato di diritto del lavoro, Padova, Cedam, 2011, p. 217 ss., G. PINo, Conflitto collettivo nei servizi pubblici essenziali, cit.; AA.VV., Diritto di sciopero e rappresentatività sindacale, Milano, Wolters Kluwer-Cedam, 2018. 
fenomeno di frammentazione della rappresentanza sindacale, della contrattazione e del conflitto che sembra rappresentare «l'aspetto saliente della sua modernizzazione» ${ }^{166}$. Con l'eventualità, peraltro, che si verifichino situazioni nelle quali su un medesimo servizio insistano soggetti economici e strutture sindacali operanti in diverse unità contrattuali ${ }^{167}$. Nel complesso, la disciplina posta dalla $1 . n$. 146/1990 rappresenta un caso emblematico di contemperamento tra autonomia ed eteronomia nello svolgimento della contrattazione collettiva, che, per il tema che ci occupa, induce a chiedersi se e fino a che punto la Commissione di garanzia sia in grado di "influenzare" le determinazioni delle parti sociali circa l'ambito di applicazione degli accordi collettivi di regolamentazione del diritto di sciopero.

\subsection{Il quadro normativo: cenni essenziali}

Che gli accordi sulle prestazioni indispensabili dovessero spiegare i propri effetti nei confronti di tutti i soggetti coinvolti nel conflitto, indipendentemente dalla natura pubblica o privata dei datori di lavoro, è parso evidente dopo l'entrata in vigore della legge n. $146 / 1990^{168}$, è stato confermato dalla Corte costituzionale ${ }^{169}$ ed è oggi pacificamente acquisito ${ }^{170}$. Tuttavia, l'attribuzione di efficacia

166 T. Treu, Il conflitto e le regole, cit., p. 314. È nel settore dei servizi pubblici essenziali, non per caso, che è emerso il fenomeno dei cd. conflitti di accreditamento (cfr. I. SECHI, Conflitto d'accreditamento: ragioni ed evidenze empiriche di una patologia, in Dir. Lav. Rel. Ind., 2017, p. 605 ss.).

167 F. SANTONI, Rappresentatività sindacale e conflitto nei servizi pubblici essenziali, in Riv. It. Dir. Lav., 2003, I, p. 331, aggiunge che «il grado di rappresentatività dei soggetti stipulanti può non corrispondere a tutte le figure professionali che assicurano il servizio nella sua globalità e complessità, qualora lo stesso ricomprenda servizi strumentali o articolati in sottoservizi che implichino assetti contrattuali diversificati».

168 M. D’Antona, Crisi e prospettive della regolamentazione extralegislativa del diritto di sciopero nei servizi pubblici essenziali, in Riv. Giur. Lav., 1991, I, p. 417 ss.

169 C. Cost. 18 ottobre 1996, n. 344, in Giur. Cost., 1996, p. 3016, Mass. Giur. Lav., 1996, p. 691, nota G. Suppiej, Dir. Lav., 1996, II, p. 421, nota N. De MARINIS.

170 C. Zoli, Contratto collettivo come fonte e contrattazione collettiva come sistema di produzione di regole, cit., pp. 496-497. Meno consenso vi è sul modo in cui tali accordi conseguono efficacia erga omnes: cfr. A. Pilati, Il campo di appli- 
erga omnes implica, per definizione, che si proceda alla «selezione di un solo negozio in ciascun ambito di riferimento (categoria od aziendale $\mathrm{o}$, al limite, territoriale) $\gg^{171}$. Peraltro, se ciò è condivisibile già su un piano di principio, questa esigenza di unitarietà di regolazione diviene invero indifferibile, in materia di sciopero nei servizi pubblici essenziali, atteso che la disciplina è assistita da un apparato sanzionatorio rigoroso ${ }^{172}$. La Commissione di garanzia lo ha rimarcato fin da alcune fondamentali delibere dei primi anni Novanta, con le quali si è stabilito che «gli standards minimi individuati dagli accordi sulle prestazioni indispensabili valutati idonei dalla Commissione di garanzia, dovendo essere unitari per ogni servizio, sono destinati ad applicarsi anche ad organizzazioni sindacali che non abbiano sottoscritto gli accordi predetti» ${ }^{173}$.

È noto che il sistema di razionalizzazione del pluralismo adottato dalla legge n. 146/1990 non muove da una selezione ex ante dei soggetti sulla base di requisiti di rappresentatività delle organizzazioni dei lavoratori o dei datori di lavoro ${ }^{174}$. Specie tra gli artt. 2, c. 2, e 13 della legge, si dipana un procedimento che consta di equilibri e sottili contrappesi, tra ruolo dell'autonomia collettiva, risoluzioni della Commissione di garanzia e intervento di enti dei consumatori e degli utenti, il cui esito auspicato è la definizione delle prestazioni indispensabili e delle altre misure di disciplinamento del conflitto ad opera degli accordi collettivi ${ }^{175}$. La ratio di tale disciplina si può ri-

cazione della legge e i requisiti di legittimità delle astensioni collettive, in F. LunARDON, Conflitto, concertazione e partecipazione, cit., pp. 344-347.

171 Così, E. Gragnoli, Sciopero nei servizi pubblici essenziali, cit., p. 785.

172 P. CuRzio, Autonomia collettiva e sciopero nei servizi essenziali, cit., pp. 160-161.

173 (Corsivo mio): Delibera 15 ottobre 1992 e Delibera 21 novembre 1991. Cfr. M.V. Ballestrero, Art. 2, in U. Romagnoli, M.V. Ballestrero, Art. 40 Supplemento Legge 12 giugno 1990 n. 146, cit., pp. 114-115.

174 Anche in questo la soluzione trovata dalla legge si distanzia dal "modello" rappresentato dall'art. 39, c. 2 ss., Cost.: non è al riguardo privo di significato che la Corte costituzionale, nella pronuncia n. 344/1996, cit. abbia escluso di poter ricondurre gli accordi sulle prestazioni indispensabili all'area di operatività della norma costituzionale; per una critica di questo argomento v. però R. Romer, Di che cosa parliamo quando parliamo di sciopero, in Lav. Dir., 1999, p. 251 ss.

175 Cfr. C. Zoli, La fruizione dei beni culturali quale servizio pubblico essenziale: il decreto legge 20 settembre 2015, $n .146$ in tema di sciopero, in Aedon, 2015, 3, p. 6 ss., ed altresì R. SANTUccI, Efficacia $o$ inadeguatezza della disciplina 
assumere nella constatazione che i soggetti delle relazioni industriali siano gli unici in grado di cogliere e di rappresentare adeguatamente le dinamiche di settore, tenendo conto dell'evoluzione dei processi produttivi e delle ragioni sostanziali dei conflitti collettivi: una legge non potrebbe intervenire con la pervasività, puntualità e dinamicità che la materia richiede ${ }^{176}$. La presenza dell'autorità indipendente dovrebbe guidare l'autonomia collettiva in questo doppio bilanciamento, tra gli interessi confliggenti delle parti stipulanti e tra questi e la sfera dei terzi coinvolti, se non dell'intera collettività ${ }^{177}$ : ciò in virtù della «conoscenza, da parte della Commissione, delle strutture e dei problemi organizzativi delle amministrazioni e delle aziende esercenti servizi pubblici essenziali», e della facoltà di «approfondire le ragioni del conflitto», nonché di compiere una serie di attività strumentali a coglierne le dinamiche ${ }^{178}$.

La dichiarazione di idoneità degli accordi collettivi ex art. 13, lett. a) - o la posizione di una regolamentazione provvisoria, in caso di mancato accordo (idoneo) - è il rimedio alla presenza di una pluralità di accordi collettivi in uno stesso ambito di applicazione. La dottrina aveva avvertito, fin dai primi anni Novanta, che quest'ultima eventualità non è teoricamente esclusa, atteso che la legge non detta una disciplina selettiva dei soggetti negoziali, ma è nella pratica improbabile. La Commissione di garanzia avrebbe, infatti, assecondato le aspirazioni negoziali solo di alcuni tra i soggetti antagonisti operanti in uno stesso settore ${ }^{179}$.

dello sciopero nei servizi pubblici essenziali (in margine allo sciopero dei docenti universitari), in Arg. Dir. Lav., 2018, p. 669.

176 A. LofFredo, La Commissione di garanzia: un'autorità indipendente tra diritto amministrativo e ordinamento intersindacale, in Lav. Dir., 2005, pp. 558-559. passim.

177 G. PINo, Conflitto collettivo nei servizi pubblici essenziali, cit., p. $272 \mathrm{ma}$

178 M. Rusciano, Sciopero, servizi essenziali, moral suasion della commissione di garanzia, in AA.VV., Diritto di sciopero e rappresentatività sindacale, cit., p. 58 .

179 G. Santoro-Passarelli, Art. 2, in M. Rusciano, G. Santoro-Passarelli (a cura di), Lo sciopero nei servizi essenziali, Milano, Giuffrè, 1991, pp. 28-29. L'autore vi ricollegava non solo il ruolo della Commissione di garanzia, bensì anche la possibilità di indire una consultazione tra i lavoratori ai sensi dell'art. 14 della legge. L'esperienza applicativa, tuttavia, ha dimostrato come quest'ultima possibilità sia stata di scarso rilievo: F. SANTONI, Rappresentatività sindacale e conflitto nei servizi pubblici essenziali, cit., p. 341. 
La disciplina di legge pone i presupposti affinché gli accordi collettivi siano «collettori di un consenso certo non generale, ma molto esteso» ${ }^{180}$, tale da assicurare la tenuta, in fatto, e la stessa credibilità del testo negoziale ${ }^{181}$. È logico che tali accordi intervengano con le organizzazioni più solide, in grado di imporre la propria presenza al tavolo negoziale ${ }^{182}$, benché la «questione [sia comunque] rimessa all'autonomia contrattuale» ${ }^{183}$.

Anche se, per l'art. 13 lett. a), la valutazione deve considerare il contenuto degli accordi in relazione all'idoneità «delle prestazioni indispensabili, delle procedure di raffreddamento e conciliazione e delle altre misure individuate ai sensi del comma 2 dell'articolo 2», gli orientamenti della Commissione di garanzia di fatto privilegiano, entro canoni di ragionevolezza, gli accordi conclusi dalle organizzazioni più rappresentative del settore ${ }^{184}$. È significativo, al proposito, che nelle deliberazioni con cui valuta idonei gli accordi collettivi del settore privato, la Commissione, con una varietà di formulazioni, dia talvolta conto del carattere (più, o anche soltanto) rappresentativo nel settore dei soggetti stipulanti ${ }^{185}$.

180 Così, R. Romer, Di che cosa parliamo quando parliamo di sciopero, cit., pp. 254-255.

181 E. Gragnol, Sciopero nei servizi pubblici essenziali, cit., p. 785.

182 Cfr. F. Borgogelli, Rappresentatività sindacale e consenso nella regolazione dello sciopero nei servizi essenziali, in Riv. Giur. Lav., 2007, I, p. 675 ss.

183 Verbale n. 1036 del 26 maggio 2014, in I. SECHI (a cura di), Commissione Garanzia Sciopero, Raccolta sistematica degli orientamenti interpretativi. I, Orientamenti di carattere generale, 2016, Capo V, XXXIV, con il quale la Commissione ha affermato che «non sussistono giustificate ragioni per un'eventuale riesame della delibera di valutazione di idoneità dell'Accordo nazionale in materia di sciopero del 18 febbraio 2013, né per un'eventuale coinvolgimento del soggetto sindacale Cisal Federenergia, questione rimessa all'autonomia contrattuale (settore elettricità)».

184 Delibera 3 giugno 1991, Delibera 18 luglio 1991. Si tenga presente che, quando tali delibere sono state emanate, la formulazione dell'art. 13 cit. era diversa da quella attualmente vigente e riportata nel testo, ma comunque tale da sollecitare la Commissione, come noto, ad operare una verifica soltanto «oggettiva» dei contenuti degli accordi sulle prestazioni indispensabili.

185 Si possono menzionare a questo proposito tre esempi: 1) la Delibera $\mathrm{n}$. 04/148 del 18 marzo 2004, con la quale è stato valutato idoneo l'accordo nazionale del 4 febbraio 2004 di regolamentazione dell'esercizio del diritto di sciopero nel settore metalmeccanico (Federmeccanica, Assistal e Fiom-Cgil, Fim-Cisl, UilmUil e, separatamente, Federmeccanica, Assistal e Fismic-Confsal e Federmeccanica, Assistal e Ugl Metalmeccanici), il cui Considerato n. 1 precisa che l'accordo «risulta sottoscritto dalle organizzazioni datoriali che rappresentano le aziende che ope- 


\subsection{La definizione del campo di applicazione degli accordi col- lettivi}

Fatte tali premesse, è possibile svolgere alcune considerazioni con riferimento più specifico ai temi della ricerca. È indubbio che, in questa materia, sia irrinunciabile la presenza di clausole negoziali che definiscano il campo di applicazione degli accordi collettivi in modo esatto, specifico e il più possibile aderente alla realtà dei processi produttivi. $\mathrm{Ne}$ va della credibilità del regime sanzionatorio e, ancor prima, dell'operatività della disciplina pattizia, che sarebbe destinata ad una scarsa effettività, se non fosse coerente con le strutture delle relazioni industriali $^{186}$.

In altri termini, è la stessa gravità dei compiti che la legge attribuisce all'autonomia collettiva (prestazioni indispensabili, procedure di raffreddamento e di conciliazione, preavviso, etc.) a rendere decisiva l'iniziativa qualificatoria. Iniziativa che, in caso di regolare andamento dei congegni previsti dalla legge n. 146/1990, è rimessa alle parti stipulanti: l'identificazione dell'area contrattuale, per questi accordi collettivi sui generis per l'esperienza delle relazioni industriali italiane, si pone, cioè, in linea con i principi ricavabili dall'art. 39, c. 1, Cost. Ciò avviene $\mathrm{o}$ in via di premesse che costituiscono parte integrante degli accordi ${ }^{187}$

rano nel settore metalmeccanico e da un insieme di organizzazioni sindacali che comprende le più significative sigle sindacali presenti nel settore»; 2) la Delibera n. 01/31 del 19 aprile 2001, con la quale è stato valutato idoneo l'accordo nazionale del $1^{\circ}$ marzo 2001 di regolamentazione dell'esercizio del diritto di sciopero per i lavoratori addetti ai servizi di igiene ambientale e/o servizi alla collettività (Federambiente, Fise e Fp-Cgil, Fit-Cisl, Uiltrasporti), il cui n. 2 della Premessa precisa che «le Associazioni datoriali Federambiente e Fise e le OO.SS. nazionali rappresentative del settore si sono incontrate numerose volte presso la sede della Commissione di garanzia»; 3) la Delibera n. 01/9 del 22 febbraio 2001, con la quale è stato valutato idoneo l'accordo nazionale del 23 gennaio 2001 di regolamentazione dell'esercizio del diritto di sciopero nel settore del credito (ABI e Fabi, Falcri, Federdirigenticredito, Fiba-Cisl, Fisac-Cgil, Sinfub, Uil C.A.), il cui Considerato n. 1 precisa che l'accordo «risulta sottoscritto dall'organizzazione datoriale che rappresenta gli istituti di credito (con la sola eccezione degli istituti affiliati in Federcasse) e da un insieme di organizzazioni sindacali, che comprende pressocché tutte le sigle sindacali presenti nel settore».

186 G. Prosperetti, La commissione di garanzia, in M. D’Onghia, M. Ricci (a cura di), Lo sciopero nei servizi pubblici essenziali, cit., p. 48.

187 Come ad esempio per l'accordo nazionale del 26 gennaio 2004 di regolamentazione dell'esercizio del diritto di sciopero nel settore della distribuzione in- 
(come talvolta accade nei contratti collettivi di categoria), o con clausole incluse nell'articolato negoziale vero e proprio ${ }^{188} \mathrm{o}$, in ogni caso, mediante le clausole che definiscono i servizi essenziali, in relazione alle quali garantire le prestazioni indispensabili ${ }^{189}$. In alcuni settori della dottrina, si è precisato che, in tal modo, l'autonomia collettiva provvede a «determinare pro parte la fattispecie di sciopero legittimo che vale in concreto nei singoli settori interessati ${ }^{190}$, id est a delineare la cornice all'interno della quale lo sciopero può svolgersi «nel rispetto delle prescrizioni introdotte direttamente dalla legge» ${ }^{191}$.

Tuttavia, il ruolo della Commissione di garanzia non si riduce a una ratifica degli esiti negoziali delle associazioni contrapposte; l'autonomia collettiva è in questo campo "guidata" dall'autorità indipendente ${ }^{192}$, in una serie di passaggi che normalmente constano di audizioni con le parti, osservazioni e rilievi sui punti più contro-

termedia farmaceutica (ADF, Federfarma Servizi, Confcommercio e Filcams-Cgil, Fisascat-Cisl, Uiltucs-Uil) (punto 2 della «Presa d'atto»: «Tale attività riguarda la fornitura dei farmaci a tutte le Farmacie private e pubbliche dislocate capillarmente su tutto il territorio nazionale, nonché Farmacie ospedaliere, ASL e suoi Presidi, Ospedali, Case di Cura e di Riposo ed altri Enti autorizzati ad erogare prestazioni farmaceutiche»), valutato idoneo con Deliberazione n. 04/267 del 15 aprile 2004 (Considerato n. 5: «nella premessa dell'Accordo è puntualmente definito il campo di applicazione della disciplina»).

188 Come ad esempio per l'accordo nazionale del 15 gennaio 2002 di regolamentazione dell'esercizio del diritto di sciopero per i lavoratori dipendenti dalle imprese di pulizia e servizi integrati/multiservizi (Confapi-Unionservizi, LegacoopAncst, Confcooperative - Federlavoro e servizi, Confindustria-Fise, Agci-Ancosel e Filcams-Cgil, Fisascat-Cisl, Uilt) (clausola 11 - Campo di applicazione), valutato idoneo con Deliberazione n. 02/22 del 7 febbraio 2002; oppure nel caso dell'accordo nazionale del $1^{\circ}$ marzo 2001, citato supra, di regolamentazione dell'esercizio del diritto di sciopero per i lavoratori addetti ai servizi di igiene ambientale e/o servizi alla collettività (clausola 12 - Campo di applicazione).

189 Per riprendere sempre uno degli esempi citati supra, v. il punto 1) dell'accordo nazionale del 4 febbraio 2004 di regolamentazione dell'esercizio del diritto di sciopero nel settore metalmeccanico, mediante il quale «è puntualmente definito il campo di applicazione della disciplina, con riferimento alle attività delle imprese che esercitano la conduzione di impianti, reti e apparecchiature analiticamente ivi indicati» (Considerato n. 2 della Delibera n. 04/148 del 18 marzo 2004, cit.), «fermo restando il potere-dovere della Commissione di intervenire nei settori che non risultassero coperti da disciplina» (Considerato n. 3).

190 M. Pedrazzoli, Qualificazioni dell'autonomia collettiva, cit., p. 561.

191 R. RomeI, Di cosa parliamo quando parliamo di sciopero, cit., p. 256.

192 Già prima della novella del 2000, v. P. CuRzio, Autonomia collettiva e sciopero nei servizi essenziali, cit., p. 103 ss. 
versi, acquisizione e considerazione dei pareri delle associazioni degli utenti e dei consumatori e così via. Lo strumentario che la legge mette a disposizione della Commissione di garanzia è volto, nel complesso, a favorire un confronto continuo con le parti sociali ${ }^{193}$.

La Commissione di garanzia è in grado di esercitare le proprie prerogative in modo penetrante, per orientare le determinazioni delle organizzazioni contrapposte anche in materia di definizione degli ambiti di applicazione degli accordi; ossia, per definire esattamente l'area dei servizi essenziali da garantire ${ }^{194}$. Al riguardo, la legge $\mathrm{n}$. $83 / 2000$ ha esteso il raggio di azione della Commissione, atteso che l'art. 13 lett. $b$ ) novellato prevede che «Nel caso in cui il servizio sia svolto con il concorso di una pluralità di amministrazioni ed imprese la Commissione può convocare le amministrazioni e le imprese interessate, incluse quelle che erogano servizi strumentali, accessori o collaterali, e le rispettive organizzazioni sindacali, e formulare alle parti interessate una proposta intesa a rendere omogenei i regolamenti di cui al comma 2 dell'articolo 2 , tenuto conto delle esigenze del servizio nella sua globalità».

Del resto, i compiti della Commissione sono tanto più delicati, quanto più, nei sottosettori dei servizi, le attività d'impresa abbiano subito processi di frammentazione organizzativa. È l'esempio, tra gli altri, delle imprese del settore del credito: esse non potrebbero erogare i servizi in assenza di una serie di attività di supporto tecnico, le quali sono state, tuttavia, a partire dagli anni Ottanta del secolo scorso, esternalizzate (per lo più) a società controllate con strumenti economico-finanziari e/o societari ${ }^{195}$. Si spiega, così, perché, in linea con la contrattazione del settore, l'accordo sulle prestazioni indispensabili che le imprese del credito sono tenute ad erogare estenda il proprio campo di applicazione «oltre che nei confronti delle aziende esercenti il servizio bancario ... anche nelle imprese che ap-

193 M. Rusciano, Diritto di sciopero e assetto costituzionale, in Riv. It. Dir. Lav., 2009, I, p. 59 ss.

194 Ad esempio, per riprendere uno dei casi citati (disciplina del settore metalmeccanico), si evince dal punto 3) della Premessa alla Delibera n. 04/148, che la Commissione aveva rappresentato alle parti che una delle questioni da risolvere per la conclusione di un accordo che potesse essere valutato idoneo era relativa «al rapporto della disciplina pattizia con le regolamentazioni vigenti in settori connessi».

195 AA.VV., Categorie professionali e contratti collettivi, cit. 
plicano i contratti collettivi nazionali di settore che svolgono attività strumentali (centri di elaborazione dati, centri servizi e Internet)» ${ }^{196}$. Dopo l'approvazione della Commissione di garanzia, nel campo di applicazione definito dalle parti stipulanti, l'accordo diventa «oggettivamente opponibile a terzi» ${ }^{197}$. Viene meno, cioè, l'esigenza di un collegamento soggettivo con le posizioni individuali; l'accordo si applica obiettivamente «a quanti sono di fatto adibiti alla erogazione del servizio pubblico essenziale» ${ }^{198}$. Ai fini dell'individuazione della disciplina applicabile in caso di sciopero, in altri termini, «deve farsi riferimento non al contratto collettivo di lavoro applicato ai dipendenti quanto piuttosto al contenuto oggettivo dell'attività svolta in relazione al soddisfacimento degli interessi costituzionalmente tutelati» $^{199}$.

Nel complesso, i procedimenti previsti dalla legge rendono improbabile la sovrapposizione tra gli ambiti di applicazione delle discipline collettive da osservare in materia di sciopero nei servizi pubblici essenziali. La Commissione sovrintende, infatti, mediante i giudizi di idoneità, alla complessiva razionalità della struttura contrattuale. Ciò è quindi improbabile, ma non per questo impossibile, soprattutto per quei sottosettori in cui vi siano più strette intersezioni e sovrapposizioni tra i processi produttivi delle imprese.

196 Accordo nazionale del 23 gennaio 2001, cit., di regolamentazione dell'esercizio del diritto di sciopero nel settore del credito ( $4^{\circ}$ trattino della Premessa); v. il Considerato n. 2) della Delibera di idoneità n. 01/9, che considera in tal modo l'area contrattuale «opportunamente estesa (tenendo conto dell'indicazione in tal senso dell'art. 13, lett. b, legge n. 146/1990, come modificata dalla legge n. 83/2000)».

197 Così, G. PINo, Conflitto collettivo nei servizi pubblici essenziali, cit., p. 274.

198 Delibera 01/31, cit.; interpellato per rendere un parere circa l'accordo stipulato per il settore igiene ambientale, il Codacons aveva sollecitato la Commissione di garanzia affinché esortasse le parti stipulanti a chiarire meglio il campo di applicazione dell'accordo, mediante un rinvio al Ccnl di settore, al fine di «individuare tutti i soggetti interessati dalla disposizione»; la Commissione (Considerato n. 8 della delibera) precisava tuttavia che «gli accordi sulle prestazioni minime si applicano erga omnes a quanti sono di fatto adibiti alla erogazione del servizio pubblico essenziale e pertanto il rinvio al CCNL non appare opportuno», respingendo la richiesta dell'associazione.

199 Verbale n. 716 del 22 marzo 2007, in I. SeCHI (a cura di), Commissione Garanzia Sciopero, Raccolta sistematica degli orientamenti interpretativi. I, Orientamenti di carattere generale, cit., XXXIII-XXXIV. 
In tali occasioni, si può fare riferimento a quanto la Commissione ha stabilito, con una delibera di orientamento adottata il 26 aprile 2006: «Con riguardo all'ipotesi di coesistenza di due accordi dichiarati idonei dalla Commissione relativi al medesimo servizio essenziale, la Commissione medesima delibera che si debba fare riferimento all'accordo sulla regolamentazione dello sciopero nei servizi pubblici essenziali corrispondente al contratto collettivo di lavoro applicato dall'azienda» ${ }^{200}$.

In caso di inerzia delle parti sociali, o di incapacità di raggiungere un accordo che la Commissione consideri idoneo, la contrattazione collettiva è privata, oltre che della prerogativa di dettare una disciplina da osservare in caso di sciopero, anche della funzione di individuarne il campo di applicazione, che è rispetto ad essa un'operazione logicamente precedente. Con la posizione di una regolamentazione provvisoria «delle prestazioni indispensabili, delle procedure di raffreddamento e di conciliazione e delle altre misure di contemperamento», giusta l'art. 13, lett. a), la Commissione provvede a individuarne d'imperio il campo di applicazione ${ }^{201}$. Diversamente, beninteso, nel caso in cui la provvisoria regolamentazione si limiti a integrare un accordo valutato parzialmente idoneo, sarà quest'ultimo a individuare il proprio campo di applicazione.

Il procedimento che può portare all'adozione di una provvisoria regolamentazione è circondato comunque di una serie di accorgimenti; si è osservato, a questo proposito, che nel consolidare questa prerogativa dell'autorità indipendente, emersa già nella vigenza della legge n. 146/1990 (formulazione originaria), il legislatore ne abbia accentuato il carattere procedurale ${ }^{202}$. La proposta formulata

200 Deliberazione n. 06/232 del 26 aprile 2006.

201 V. ad esempio art. 1 - Campo di applicazione, della Delibera n. 06/431 del 19 luglio 2006, che detta la provvisoria regolamentazione delle prestazioni indispensabili e delle altre misure di cui all'art. 2, comma 2, 1. n. 146/1990, come modificata dalla 1. n. 83/2000, per il settore della vigilanza, sicurezza e ordine pubblico, «nella parte in cui è funzionale e/o strumentale ai diritti costituzionalmente tutelati...» (Considerato n. 1).

202 M. Rusciano, Diritto di sciopero e assetto costituzionale, cit., p. 60. È significativo, a tale proposito, che la disciplina novellata abbia, da un lato, introdotto una serie di parametri per guidare la valutazione della Commissione e, dall'altro lato, sollecitato la stessa a prendere in considerazione le previsioni degli atti di autoregolamentazione vigenti in settori analoghi o similari nonché gli accordi sotto- 
dalla Commissione deve, infatti, essere notificata alle parti interessate, le quali possono pronunciarsi in un termine di quindici giorni; trascorso il quale, la Commissione è tenuta a indire alcune audizioni, da svolgersi entro un termine di venti giorni. A quel punto, «l'indisponibilità delle parti a raggiungere un accordo» può giustificare l'adozione di una provvisoria regolamentazione, «comunicandola alle parti interessate, che sono tenute ad osservarla agli effetti dell'articolo 2, comma 3, fino al raggiungimento di un accordo valutato idoneo». A ben vedere una lettura delle delibere con le quali sono adottate le provvisorie regolamentazioni conferma che per la Commissione si tratti di un'extrema ratio, da percorrere se vi sia la certezza che un accordo non può essere raggiunto ${ }^{203}$ : del resto, «l'attività della Commissione, in linea con lo spirito della legge, è stata sempre rivolta a favorire un sistema autoregolato di conflitto» ${ }^{204}$. Né si può trascurare che, in questa materia, graverebbe sulle parti un onere di trattare in buona fede, che dovrebbe scongiurare prassi consistenti nel rifiuto tout court di partecipare al tavolo negoziale ${ }^{205}$.

Riepilogando, dalle riflessioni svolte possono trarsi alcune considerazioni rilevanti. La 1. n. 146/1990 attribuisce alle parti sociali il compito di stabilire, nei contratti collettivi, sia pure sotto la "guida" della Commissione di garanzia, le regole sull'esercizio del diritto di sciopero nel settore nevralgico dei servizi pubblici essenziali. Per come vengono ad esistenza, tali discipline collettive non soltanto sono in grado di spiegare i propri effetti nei confronti di tutti i soggetti oggettivamente ricompresi nel campo di applicazione, ma è altre-

scritti nello stesso settore dalle organizzazioni sindacali comparativamente più rappresentative sul piano nazionale.

203 Si veda a questo proposito la Delibera n. 06/431, cit., che nei Considerato 1-11 ripercorre sinteticamente tutti i passaggi che hanno condotto all'adozione della provvisoria regolamentazione. In giurisprudenza, Cons. st., Sez. VI, 11 marzo 2010, n. 1437, che in relazione alla revisione della regolamentazione provvisoria nel settore delle telecomunicazioni afferma che «L'intervento della Commissione è giustificato dalla inerzia delle parti collettive, protrattasi nel tempo nonostante le intenzioni più volte manifestate in merito al superamento del regime di etero-regolazione del conflitto sindacale».

204 Così, la Relazione del Presidente Giuseppe Santoro-Passarelli per l'anno 2018, Roma, 18 giugno 2019, p. 4.

205 C. Zoli, Gli obblighi a trattare nel sistema dei rapporti collettivi, Padova, Cedam, 1992, p. 286 ss. 
sì improbabile che si sovrappongano tra loro. Questo è il punto di maggiore interesse, nel quale prende pienamente corpo quell'equilibrio tra autonomia ed eteronomia che è il tratto saliente della legge del 1990 (per come novellata nel 2000): la prerogativa di definire il campo di applicazione degli accordi collettivi, che è gelosamente difesa dalle parti sociali, in quanto espressione diretta di libertà sindacale, è ad esse, come di consueto, attribuita, ma, al contempo, la presenza della Commissione di garanzia consente alla struttura contrattuale di conservare una adeguata razionalità ed efficienza. Tuttavia, in caso di mancato raggiungimento di un accordo idoneo, il venir meno, in tutto o in parte, della prerogativa delle parti sociali di definire l'ambito di operatività delle regole sul diritto di sciopero è una conseguenza del procedimento, che se le priva, sia pure pro tempore, della possibilità di stabilirne il contenuto, può implicare altresì l'eventualità che esse siano costrette a muoversi su un "campo di gioco" imposto dall'alto. 


\section{CAPITOLO IV \\ PARAMETRI DEL TRATTAMENTO E RINVII ALL'AUTONOMIA COLLETTIVA}

Sommario: 1. Parametro del trattamento economico e normativo e rinvii all'autonomia collettiva per determinarlo. - 2. Un modello di intervento "leggero" sul sistema sindacale. -3 . Le clausole sul campo di applicazione dei contratti collettivi: problemi interpretativi. - 4. La selezione dei contratti-parametro: il problema di un ambito residuo di vigenza dell'art. 2070 c.c. - 5. Pluralità di contratti collettivi con ambito di applicazione sovrapposto. L'esigenza di dotarsi di più adeguati criteri selettivi. - 6. Selezioni e misurazioni nella maggior rappresentatività comparata. - 6.1. La comparazione tra contratti collettivi con ambito di applicazione esattamente coincidente. - 6.1.1. Critica. - 6.1.2. Spunti per una ricostruzione alternativa. - 6.2. La misurazione effettiva della rappresentatività: un cantiere aperto nelle relazioni industriali. -6.3 . Le rilevazioni Cnel-Inps sull'applicazione dei contratti collettivi alle imprese e ai lavoratori ad esse addetti.

1. Parametro del trattamento economico e normativo e rinvii all'autonomia collettiva per determinarlo

All'abrogazione dell'ordinamento corporativo nel 1943-1944 seguì l'impossibilità di continuare a produrre il diritto professionale che per quasi due decenni aveva regolato i rapporti di lavoro. In attesa di dare una sistemazione organica all'ordinamento sindacale democratico ${ }^{1}$, per evitare i rischi di un vuoto di disciplina, furo-

1 V. per un quadro M. GRANDI, I problemi del lavoro negli orientamenti della dottrina giuridica: organizzazione sindacale, contratto collettivo e sciopero, cit. 
no previste la possibilità di recepire in atti dell'esecutivo gli accordi che sarebbero stati stipulati dalle organizzazioni sindacali, affidate temporaneamente ad esponenti dell'antifascismo (art. 4, r.d.l. n. 721/1943) e il mantenimento in vigore dei contratti collettivi corporativi (art. 43, d.lgs. lt. n. 369/1944) ${ }^{2}$. È noto che dopo l'entrata in vigore della Costituzione repubblicana un'organica sistemazione dell'ordinamento sindacale per via legislativa non sia mai intervenuta. A ciò contribuirono una serie di ragioni, legate ai rapporti interni al movimento sindacale e al quadro politico del dopoguerra, oltre che di redazione del testo della norma costituzionale, sulle quali la dottrina ha da tempo fatto chiarezza ${ }^{3}$. Benché dall'art. 39 Cost. fosse ricavabile una "direzione normativa di massima", tesa a conciliare il pluralismo sindacale con l'attribuzione di efficacia generale ai contratti collettivi, in assenza di una legge attuativa il risultato non poteva essere conseguito ${ }^{4}$.

Di ciò si sarebbero in qualche modo fatti carico la giurisprudenza e il legislatore, con una serie di soluzioni volte ad estendere gli effetti dei contratti collettivi di diritto comune 5 .

Fin dai primi anni Cinquanta del secolo, in giurisprudenza si affermava un orientamento per il quale il contratto collettivo di diritto comune può costituire valida fonte di completamento di norme a contenuto precettivo ricavabili dall'ordinamento giuridico ${ }^{6}$. Il più importante esempio di ciò è costituito dalla giurisprudenza sull'art. 36 Cost., che trasferisce, sia pure indirettamente, nel diritto ${ }^{7}$, il compromesso salariale accettato dalle forze sociali in ciascuna unità contrattuale e, tramite i sistemi di classificazione e di inquadramento, per ogni o quasi attività di lavoro dipendente.

2 L. Riva SAnseverino, Il contratto collettivo nella legislazione italiana, in Dir. Lav., 1946, I, p. 5.

3 Per tutti, G. Giugni, Art. 39, cit.

4 L. Mengoni, Le fonti del diritto del lavoro in Italia, cit., p. 148.

5 Una trattazione sintetica in A. Lo FARO, Contratto collettivo (lavoro privato), cit., p. 196 ss.

6 Una ricostruzione complessiva della tematica fu all'epoca tentata da C. AsSANTI, Rilevanza e tipicità del contratto collettivo nella vigente legislazione italiana, Milano, Giuffrè, 1967.

7 Per lo svolgimento metodologico della giurisprudenza come canale di comunicazione tra ordinamento statale e ordinamento intersindacale, v. G. GIUGNI, Introduzione allo studio dell'autonomia collettiva, cit., p. 71 ss. 
Secondo tale orientamento, la retribuzione complessivamente corrisposta ai lavoratori non può essere inferiore ai minimi tabellari previsti dai contratti collettivi, a pena di violazione dell'art. 36 Cost. e conseguente nullità della disposizione del contratto individuale o collettivo dal quale si determini la retribuzione ${ }^{8}$. In tal caso, per stabilire la retribuzione costituzionalmente adeguata, il giudice farà riferimento, come metro di valutazione ${ }^{9}$, ai minimi tabellari del contratto collettivo del settore di attività in cui opera il datore di lavoro $^{10}$, oppure a quello di un settore affine, in caso di «impossibilità di fare riferimento a un contratto collettivo stipulato per la specifica categoria a cui appartiene l'impresa» ${ }^{11}$.

Questa operazione giurisprudenziale, dalla valenza «trasgressiva nel contesto d'un rapporto giuridico civile» ${ }^{12}$, avrebbe acquisito un rilievo sistematico per la materia ${ }^{13}$. È significativo che negli studi comparati dedicati alla contrattazione collettiva l'ordinamento italiano sia sovente etichettato tra quelli privi di un meccanismo formale di estensione degli effetti dei contratti collettivi, ma nel quale, al contempo, l'applicazione tendenzialmente generale dei minimi salariali previ-

8 Cfr. S. Bellomo, Art. 36, in G. Amoroso, V. Di Cerbo, A. Maresca (a cura di), Il diritto del lavoro, 1, La Costituzione, il Codice civile e le leggi speciali, V ed., cit., p. 164 ss., L. Nogler, S. Brun, Art. 36, in R. De Luca Tamajo, O. Mazzotta (a cura di), Commentario breve alle leggi sul lavoro, VI ed., cit., p. 40 ss. Nei casi in cui la retribuzione sia determinata da un contratto collettivo, al giudice è richiesta particolare prudenza nella valutazione, onde evitare che l'autorità giudiziaria finisca per sostituirsi sistematicamente alle parti sociali nella determinazione dei livelli salariali di mercato (L. IMBERTI, Art. 36 Costituzione: in assenza di interventi legislativi chi è l'autorità salariale?, in Lav. Dir. Eur., 2019/3).

9 M. Rusciano, Il contratto collettivo, cit., p. 65 ss.

10 Tra le moltissime rinvenibili, v. Cass. 25 febbraio 1994, n. 1903, in Riv. Giur. Lav., 1994, II, p. 409 ss., nota C. De MARCHIs, Cass. 4 giugno 2008, n. 14791, in Lav. Giur., 2008, p. 1167. È noto che la giurisprudenza ammette talora che si possa fare riferimento ai contratti collettivi aziendali (v. Cass. 2 agosto 2018, n. 20452), ma ciò non ha particolare rilievo per i ragionamenti svolti nel testo.

11 Si trae da Cass. 17 marzo 2000, n. 3184, in Dir. Prat. Lav., 2000, p. 41, ma v. altresì Cass. 27 maggio 1995, n. 5970, in Giust. Civ. Mass., 1995, p. 1097, Cass. 23 dicembre 2005, n. 28655, in Pluris.

12 Così, L. Montuschi, La Costituzione e i lavori, in Riv. It. Dir. Lav., 2009, I, p. 160.

13 O. Mazzotta, Il diritto del lavoro e le sue fonti, in Riv. It. Dir. Lav., 2001, I, p. 224. 
sti dai contratti collettivi è assicurata dalla giurisprudenza ${ }^{14}$. Scriveva Treu nel 1979 che questo orientamento è «l'altra faccia del fallimento dell'ordinamento contrattuale ipotizzato dal Costituente nell'art. $39 »^{15}$.

Peraltro, all'orientamento che poggia sull'art. 36 Cost., si andavano via via affiancando una serie di altre soluzioni giurisprudenziali, nelle quali ugualmente i contratti collettivi erano utilizzati come criterio di riferimento per il completamento operativo di disposizioni di legge. È il caso delle norme contenute negli artt. $2095^{16}, 2106^{17} \mathrm{e}$ $2110^{18}$ c.c., per non citare che alcuni esempi particolarmente significativi $^{19}$, nei quali il richiamo alle «norme corporative» è interpretato come un rinvio indirizzabile altresì ai contratti collettivi di diritto comune. Più che soffermarvisi giova rimarcare in che modo, nella legislazione speciale, si sia gradualmente affermata la tecnica del rinvio ai

14 S. HAYTER, J. VISSER, The application and extension of collective agreements: Enhancing the inclusiveness of labour protection, in S. HAYTER, J. VISSER (eds), Collective Agreements: Extending Labour Protection, cit., p. 11, table 1.1.

15 T. Treu, Art. 36, in Giu. Branca (a cura di), Commentario della Costituzione. Rapporti Economici, I, cit., p. 79. Cfr. altresì M.G. GAROFAlo, Attività creatrice del giudice e ricerca sui fatti, in Pol. Dir., 1972, p. 265.

16 In materia di categorie dei prestatori di lavoro, peraltro, l'integrazione tra legge e contrattazione collettiva è stata rafforzata dalla 1. n. 190/1985, che all'art. 2 , c. 2 , ha stabilito che «I requisiti di appartenenza alla categoria dei quadri sono stabiliti dalla contrattazione collettiva nazionale o aziendale in relazione a ciascun ramo di produzione e alla particolare struttura organizzativa dell'impresa».

17 S. MaInARDI, Il potere disciplinare nel lavoro privato e pubblico, Milano, Giuffrè, 2002. La legislazione speciale ha di recente rafforzato l'integrazione tra legge e contrattazione collettiva in questa materia (cfr. I. Alvino, I rinvii legislativi al contratto collettivo, Napoli, Jovene, 2018, pp. 97-99): dapprima, con la 1. n. 183/2010 (art. 30, c. 3), si è stabilito che «Nel valutare le motivazioni poste a base del licenziamento, il giudice tiene conto delle tipizzazioni di giusta causa e di giustificato motivo presenti nei contratti collettivi di lavoro stipulati dai sindacati comparativamente più rappresentativi»; in seguito, con la riforma (art. 1, c. 42, 1. n. 92/2012) dell'art. 18 dello statuto dei lavoratori, è stato previsto che (c. 4), in caso di licenziamento illegittimo, il rimedio della tutela reintegratoria con indennità economica limitata a 12 mensilità sia disposto dal giudice anche nei casi in cui il fatto contestato al lavoratore «rientra tra le condotte punibili con una sanzione conservativa sulla base delle previsioni dei contratti collettivi».

18 R. Del Punta, La sospensione del rapporto di lavoro. Malattia, infortunio, maternità, servizio militare. Artt. 2110-2111, Milano, Giuffrè, 1992, p. 248.

19 Un quadro più ampio in R. Scognamiglio, Il codice civile e il diritto del lavoro, in AA.VV., Le ragioni del diritto. Scritti in onore di Luigi Mengoni, II, Milano, Giuffrè, 1995, pp. 1253-1254. 
contratti collettivi per la definizione di un parametro del trattamento economico e/o normativo dovuto ai lavoratori.

Le prime esperienze di rinvio legale ai contratti collettivi possono apparire, per così dire, "rudimentali”, per una redazione delle proposizioni normative tale da far sorgere il dubbio di aver stabilito surrettiziamente l'obbligatorietà erga omnes dei contratti collettivi, con congegni diversi da quelli previsti dall'art. 39 Cost. ${ }^{20}$. Tuttavia, sia la disciplina del collocamento del $1949^{21}$ sia quella dell'apprendistato del $1955^{22}$ furono interpretate, dalla dottrina ${ }^{23}$ e dalla giurisprudenza ${ }^{24}$ prevalenti, in modo compatibile con quella che anni dopo sarebbe stata definita rilevanza negativa ${ }^{25}$ o ostativa ${ }^{26}$ dell'art. 39 Cost.

Esempi più calzanti possono essere tratti dalle discipline sulle lavoratrici madri $(1950)^{27}$ e, soprattutto, sul lavoro a domicilio

20 M. Rusciano, Il contratto collettivo, cit., p. 66, nota 5, allude a fragili tentativi «di fondare l'efficacia erga omnes del contratto collettivo di diritto comune su norme di legge che, regolando tutt'altra materia, genericamente alludono ai "contratti collettivi" e che, dal tenore letterale e dalla ratio, presuppongono se mai per la loro corretta applicazione proprio l'esistenza di contratti efficaci erga omnes».

21 Art. 14, c. 4, 1. n. 264/1949: «L'Ufficio di collocamento, nell'atto di soddisfare la richiesta del datore di lavoro, è tenuto ad accertarsi che le condizioni offerte ai nuovi assunti siano conformi alle tariffe e ai contratti collettivi».

22 Art. 11, lett. c), 1. n. 25/1955: «Il datore di lavoro ha l'obbligo: [...] di osservare le norme dei contratti collettivi di lavoro e di retribuire l'apprendista in base ai contratti stessi» (l'art. 23, lett. b) comminava una sanzione pecuniaria per la violazione di tale disposizione).

23 Cfr. L. Montuschi, I limiti legali nella conclusione del contratto di lavoro, Milano, Giuffrè, 1964, pp. 293-294 (in materia di collocamento), G. SuPPIEJ, Pluralismo dei contratti collettivi e significato di un rinvio legislativo, in Riv. Dir. Lav., 1957, II, p. 212 ss. (in materia di apprendistato).

24 Cass. 21 novembre 1977, n. 5081, in Riv. Giur. Lav., 1978, II, p. 926 ss., nota P. Ichino, Cass. 27 maggio 1983, n. 3678, in Mass. Giur. It., 1983, n. 5 (in materia di collocamento); C. cost. 18 gennaio 1957, n. 10, in Giur. Cost., 1957, p. 72 ss., nota C. Esposito (in materia di apprendistato).

${ }_{25}$ Ad esempio, L. MARIUCCI, La contrattazione collettiva, Bologna, Il Mulino, 1985, p. 304.

26 B. Caruso, A. Lo Faro, Contratto collettivo di lavoro (Voce per un dizionario), in WP CSDLE “Massimo D'Antona”.IT, 97/2010, p. 7.

27 Art. 15, 1. n. 860/1950: «In caso di dimissioni volontarie presentate durante il periodo per cui è previsto, a norma del precedente art. 3 il divieto di licenziamento, la lavoratrice ha diritto alle indennità previste da disposizioni di legge e contrattuali per il caso di licenziamento». 
del $1958^{28}$ e del $1973^{29}$ : quest'ultima stabiliva che i lavoratori «debbono essere retribuiti sulla base di tariffe di cottimo pieno risultanti dai contratti collettivi della categoria». È significativo che tale disposizione sia stata ricollegata all'orientamento giurisprudenziale sull'art. 36 Cost., sottolineandone il significato di «tipizzazione» ${ }^{30}$ per via legislativa, volta ad assicurare speciale protezione ad una categoria a rischio di esclusione dal cono d'ombra del lavoro organizzato. Si escludeva, perciò, la violazione dell'art. 39 Cost.: il rinvio operato dalla legge non attribuiva efficacia obbligatoria erga omnes ai contratti collettivi ${ }^{31}$; in attuazione dell'art. 35 Cost. $^{32}$ si limitava a porre un parametro per la misura del trattamento economico, traendolo dai contratti collettivi liberamente conclusi dalle parti sociali ${ }^{33}$.

Riordinando una serie di previsioni più risalenti e superandone il carattere settoriale ${ }^{34}$, l'art. 36 dello statuto dei lavoratori disponeva che nei provvedimenti di concessione di benefici alle imprese da parte dello Stato e nei capitolati di appalto per opere pubbliche ${ }^{35}$ fossero inserite clausole aventi ad oggetto l'obbligo «di applicare o di far applicare nei confronti dei lavoratori dipendenti condizioni non inferiori a quelle risultanti dai contratti collettivi di lavoro della categoria e della zona». Il trattamento economico e normativo dei contratti collettivi di livello nazionale o provinciale ${ }^{36}$ era richiamato quale «termine di raffronto» ${ }^{37}$. Il

\footnotetext{
28 Art. 6, 1. n. 264/1958.

29 Art. 8, c. 1, 1. n. 877/1973.

30 L. MARIUCCI, Il lavoro decentrato: discipline legislative e contrattuali, Milano, Franco Angeli, 1979, p. 132.

31 L. Nogler, Lavoro a domicilio. Art. 2128, Milano, Giuffrè, 2000, pp. 325326, spec. nota 57 , anche per i richiami di dottrina.

32 C. cost. 7 febbraio 2000, n. 49, in Mass. Giur. Lav., 2000, p. 237 ss., nota R. Pessi.

33 D. Garofalo, Il lavoro a domicilio, in Riv. It. Dir. Lav., 2018, III, p. 140, sottolinea la finalità di garantire la parità di trattamento con i dipendenti "interni" dell'azienda.

34 Cfr. P. Tulunn, Finanziamenti pubblici alle imprese e «clausole sociali», in Riv. Trim. Dir. Proc. Civ., 1990, p. 39 ss.

35 C. cost. 19 giugno 1998, n. 226, in Giust. Civ., 1998, p. 2423, nota G. PERA, ha ampliato l'ambito di applicazione della norma, includendovi le concessioni di servizi pubblici.

36 È più incerto se la norma comprendesse anche i contratti collettivi di livello aziendale: cfr. D. IzzI, Lavoro negli appalti e dumping salariale, cit., pp. 75-76.

37 Così, E. Ghera, Le c.d. clausole sociali: evoluzione di un modello di politica legislativa, cit., p. 135.
} 
rispetto di tali trattamenti si configurava come meccanismo di incentivo all'ingresso dei datori di lavoro nel sistema contrattuale, ossia come contropartita per la concessione di benefici o per il conseguimento di un appalto pubblico ${ }^{38}$ : ciò consentiva di escludere la violazione dell'art. 39 Cost. $^{39}$. La norma statutaria contribuiva, così, ad elevare il tasso di applicazione dei contratti collettivi nel tessuto produttivo ${ }^{40}$, al tempo stesso livellando al rialzo la concorrenza tra le imprese, riducendo i rischi che il mercato fosse interessato da agitazioni sindacali ${ }^{41}$ e favorendo, in definitiva, la qualità dei servizi offerti ${ }^{42}$.

È noto che le discipline relative ai contratti pubblici e ai benefici alle imprese ${ }^{43}$ sono state riviste più volte dal legislatore. Per i contratti pubblici, le discipline in vigore si ricavano dal d.lgs. $n$. 50/2016, le cui disposizioni di rinvio all'autonomia collettiva sono assai più articolate ed incisive, rispetto a quanto previsto dalla norma statutaria.

Limitandosi a un richiamo superficiale ${ }^{44}$, vengono anzitutto in rilievo le disposizioni sulla determinazione del costo del lavoro per i diversi settori di attività interessati da contratti pubblici (art. 23, c.

38 Cfr. G.G. Baland, Le «clausole a favore dei lavoratori» e l'estensione dell'applicazione del contratto collettivo, in Riv. Trim. Dir. Proc. Civ., 1973, p. 698 ss.

39 C. Cost. 16 luglio 1987, n. 270, in Foro It., 1988, I, 1064, nota G. PINO, in materia di concessione di benefici pubblici mediante fiscalizzazione degli oneri sociali.

40 G.F. Mancini, Art. 36, in G. Ghezzi, G.F. Mancini, L. Montuschi, U. Romagnoli, Statuto dei lavoratori, in A. Scialoja, Giu. Branca (a cura di), Commentario del codice civile, Bologna-Roma, Zanichelli-Soc. ed. del Foro italiano, 1972, p. 542 ss.

41 In un'ottica di buon andamento delle pubbliche amministrazioni (art. 97 Cost.): C. cost. n. 226/1998, cit.

42 Di recente (applicabile ratione temporis il d.lgs. n. 163/2006), in tal senso, Cons. St., Sez. III, 15 maggio 2017, n. 2252, in Pluris.

43 È oggi previsto che «i benefici normativi e contributivi previsti dalla normativa in materia di lavoro e legislazione sociale» siano legati al «rispetto degli accordi e contratti collettivi nazionali nonché di quelli regionali, territoriali o aziendali, laddove sottoscritti, stipulati dalle organizzazioni sindacali dei datori di lavoro e dei lavoratori comparativamente più rappresentative sul piano nazionale» (art. 1, c. 1175, 1. n. 296/2006).

44 Per una trattazione più approfondita, D. CAFFIO, Appalto, costo del lavoro e contratto collettivo di riferimento, in D. Garofalo (a cura di), Appalti e lavoro, I, Disciplina pubblicistica, Torino, Giappichelli, 2017, p. 849 ss. 
16). Ciò avviene, con cadenza annuale, mediante la pubblicazione da parte del Ministero del lavoro di decreti direttoriali contenenti «apposite tabelle», elaborate «sulla base dei valori economici definiti dalla contrattazione collettiva nazionale tra le organizzazioni sindacali e le organizzazioni dei datori di lavoro comparativamente più rappresentativi, delle norme in materia previdenziale e assistenziale, dei diversi settori merceologici e delle differenti aree territoriali»; in caso di assenza di contratti collettivi si può fare riferimento a quelli «del settore merceologico più vicino a quello» per il quale si determina il costo del lavoro. Le tabelle sono adoperate dalle amministrazioni in sede di progettazione dell'appalto e di selezione delle offerte (spec. art. 97).

In secondo luogo, il codice dei contratti pubblici prevede (art. 30 , c. 4) che al personale «è applicato il contratto collettivo nazionale e territoriale in vigore per il settore e per la zona nella quale si eseguono le prestazioni di lavoro stipulato dalle associazioni dei datori di lavoro e dei prestatori di lavoro comparativamente più rappresentative sul piano nazionale e quelli il cui ambito di applicazione sia strettamente connesso con l'attività oggetto dell'appalto o della concessione svolta dall'impresa anche in maniera prevalente». Per la sua formulazione, la norma non sembra, invero, limitarsi ad imporre l'osservanza di un parametro minimo di trattamento ricavato dai contratti collettivi: se intesa in senso letterale, essa dispone che i contratti collettivi siano applicati tout court ${ }^{45}$. Al proposito, è condivisibile l'opinione secondo la quale ciò non si risolverebbe in una violazione dell'art. 39 Cost.: l'applicazione dei contratti collettivi deriva pur sempre dalla scelta in sé libera di entrare in rapporti negoziali con il pubblico ${ }^{46}$, oltre che da un'inevitabile compressione della libertà contrattuale delle parti che, in

45 D. Izzi, Lavoro negli appalti e dumping salariale, cit., pp. 78-81, 107-109, B. De Mozzi, Terzo settore e contrattazione collettiva, in Variaz. Temi Dir. Lav., 2019, p. 1039.

46 Cfr., in relazione al codice dei contratti pubblici del 2006, S. Varva, Le clausole sociali, in M.T. Carinci, C. Cester, M.G. Mattarolo, F. Scarpelli (a cura di), Tutela e sicurezza del lavoro negli appalti privati e pubblici. Inquadramento giuridico ed effettività, Torino, Utet, 2011, p. 342; nello stesso senso, dopo l'entrata in vigore della disciplina del 2015, D. IzzI, Lavoro negli appalti e dumping salariale, cit., pp. 78-79. 
questa materia, finalità di limitazione del dumping sociale giustificano ampiamente ${ }^{47}$.

In relazione a lavori e servizi cd. ad alta intensità di manodope$\mathrm{ra}^{48}$, oltre ad una clausola di stabilità occupazionale ${ }^{49}$, il codice prevede (art. 50) l'inserimento in bandi di gara, avvisi e inviti di clausole che prevedano «l'applicazione da parte dell'aggiudicatario, dei contratti collettivi di settore di cui all'art. 51 del decreto legislativo 15 giugno 2015, n. $81 »^{50}$. Inoltre, in relazione ai cd. subappalti, l'art. 105, c. 9, del codice prevede che «L'affidatario è tenuto ad osservare integralmente il trattamento economico e normativo stabilito dai contratti collettivi nazionale e territoriale in vigore per il settore e per la zona nella quale si eseguono le prestazioni».

Si può menzionare, altresì, la 1. n. 142/2001 (art. 3, c. 1) in materia di società cooperative. Tali società «sono tenute a corrispondere al socio lavoratore un trattamento economico complessivo proporzionato alla quantità e qualità del lavoro prestato e comunque non inferiore ai minimi previsti, per prestazioni analoghe, dalla contrattazione collettiva nazionale del settore o della categoria affine» ${ }^{51}$. La disciplina è stata integrata nel 2007: «in presenza di una pluralità di contratti collettivi della medesima categoria», le cooperative applicheranno «trattamenti economici complessivi non inferiori a quelli dettati dai contratti collettivi stipulati dalle organizzazioni datoriali e sindacali comparativamente più rappresentative a livello nazionale nella categoria» ${ }^{52}$. In un settore del mercato che si è rivelato

47 P. TullinI, Concorrenza ed equità nel mercato europeo: una scommessa difficile (ma necessaria) per il diritto del lavoro, in Riv. It. Dir. Lav., 2018, I, pp. 209-210.

48 In base all'art. 50, sono tali quelli nei quali il costo della manodopera rappresenti almeno il $50 \%$ del costo totale del contratto.

49 L. RatTI, Autonomia collettiva e tutela dell'occupazione. Elementi per un inquadramento delle clausole di riassunzione nell'ordinamento multilivello, Milano, Wolters Kluwer-Cedam, 2018.

50 L'art. 51, d.lgs. n. 81/2015 prevede che per contratti collettivi si debbano intendere $\ll i$ contratti collettivi nazionali, territoriali o aziendali stipulati da associazioni sindacali comparativamente più rappresentative sul piano nazionale e i contratti collettivi aziendali stipulati dalle loro rappresentanze sindacali aziendali ovvero dalla rappresentanza sindacale unitaria».

51 Art. 3, 1. n. 142/2001.

52 Art. 7, c. 4, d.1. n. 248/2007, conv. con modifiche 1. n. 31/2008. 
esposto a fenomeni distorsivi della concorrenza, attuati anche per via dell'applicazione di contratti collettivi sottoscritti da organizzazioni dei lavoratori e dei datori di lavoro scarsamente rappresentative $^{53}$, la legge ha rafforzato la garanzia prevista dall'art. 36 Cost. $^{54}$. È un altro esempio, cioè, di tipizzazione per via legislativa della giurisprudenza sulla retribuzione costituzionalmente adeguata, atteso che è imposto alle cooperative un obbligo di osservare i trattamenti economici previsti dai contratti collettivi come metro di misura delle proprie determinazioni ${ }^{55}$. Ciò consente di affermarne la compatibilità con l'art. 39 Cost..$^{56}$.

Altri esempi possono trarsi da recenti interventi del legislatore, a conferma della tendenza - che parrebbe "espansiva”, dopo la sentenza n. 51/2015 della Corte costituzionale - ad un uso più robusto di tale tecnica regolativa ${ }^{57}$. È il caso degli artt. 13, d.lgs. n.

\footnotetext{
53 V. il Protocollo d'intesa 10 ottobre 2007 stipulato tra Ministero del lavoro, Ministero dello sviluppo economico, Confcooperative, Legacoop, Cgil, Cisl e Uil.

54 C. cost. 26 marzo 2015, n. 51, in Arg. Dir. Lav., 2015, II, p. 928 ss., nota S. Laforgia; in Riv. Giur. Lav., 2015, II, p. 493 ss., nota M. Barbieri; in Dir. Rel. Ind., 2015, p. 823 ss., nota D. Schiuma.

55 Cfr. M. Tremolada, Lavoro nelle cooperative, in Enc. Dir., Annali, VII, 2014, p. 637; M.G. GReCo, Contrattazione collettiva, contratti pirata e regolamenti nella determinazione dei livelli retributivi nelle cooperative di produzione e lavoro, cit., p. 369.

56 C. cost. n. 51/2015, cit. Nella giurisprudenza di legittimità, v. Cass. n. 4951/2019, cit., Cass. n. 5189/2019, cit., Cass. n. 6143/2019, cit., Cass. 12 marzo 2019, n. 7047, Cass. 18 aprile 2019, n. 10851, Cass. 4 novembre 2019, n. 28289, tutte in DeJure. In dottrina, cfr. L. IMBERTI, Il socio lavoratore di cooperativa. Disciplina giuridica ed evidenze empiriche, Milano, Giuffrè, 2012, pp. 191-192.

57 Valorizzandone il «nucleo duraturo» (M. BARBIERI, In tema di legittimità costituzionale del rinvio al Ccnl delle organizzazioni più rappresentative nel settore cooperativo per la determinazione della retribuzione proporzionata e sufficiente, in Riv. Giur. Lav., 2015, II, p. 499), una parte della dottrina ha sviluppato le argomentazioni della Corte costituzionale oltre l'ambito di disciplina del lavoro dei soci lavoratori di cooperative. Ad esempio, G. OrLandini, Legge, contrattazione collettiva e giusta retribuzione, in Lav. Dir., 2018, pp. 14-15, valorizza l'acquis della sentenza al fine di ribadire la legittimità costituzionale e l'incisività dei rinvii all'autonomia collettiva nelle discipline sugli appalti pubblici e sul lavoro prestato nel terzo settore. Similmente, F. Borgogelli, Appalti pubblici e dumping salariale: un caso di subordinazione dell'autonomia collettiva, in Lav. Dir., 2016, pp. 987-988, D. IzzI, Lavoro negli appalti e dumping salariale, cit., p. 109 e D. CAFFIO, Appalto, costo del lavoro e contratto collettivo di riferimento, cit., p. 861 ss., occupandosi degli appalti pubblici. Infine, in un ragionamento de jure condendo, P. PAscuccI, Giusta
} 
112/2017, in materia di impresa sociale e 16, d.lgs. n. 117/2017, in materia di enti del terzo settore ${ }^{58}$ : tali disposizioni prevedono che i lavoratori «hanno diritto ad un trattamento economico e normativo non inferiore a quello previsto dai contratti collettivi di cui all'art. 51 del decreto legislativo 15 giugno 2015, n. 81». Il nesso logico con l'art. 3, c. 1, 1. n. 142/2001 è evidente, benché via sia una differenza sostanziale: nelle normative del 2017, il rinvio opera non soltanto per i trattamenti economici, ma anche per quelli normativi. I dubbi di legittimità costituzionale che, sotto tale profilo, le disposizioni sollevano ${ }^{59}$ possono essere superati, se si condivide l'opinione che, per gli enti del terzo settore e per le imprese sociali (diverse dalle cooperative sociali), l'applicazione di trattamenti normativi non inferiori a quelli previsti dai contratti collettivi deriverebbe, pur sempre, dalla scelta di usufruire del regime agevolato previsto per tali soggetti ${ }^{60}$.

Un rinvio all'autonomia collettiva è contenuto, altresì, nell'art. 20, 1. n. 81/2017, ove si prevede che il «lavoratore che svolge la prestazione in modalità di lavoro agile ha diritto ad un trattamento economico e normativo non inferiore a quello complessivamente applicato, in attuazione dei contratti collettivi di cui all'art. 51, del decreto legislativo 15 giugno 2015, n. 81, nei confronti dei lavoratori che svolgono le medesime mansioni esclusivamente all'interno

retribuzione e contratti di lavoro, oggi, in Dir. Lav. Rel. Ind., 2019, pp. 76-77, vi fonda una proposta di intervento legislativo «minimalista», che allargherebbe all'intero mercato del lavoro l'operatività dei rinvii ai trattamenti economici previsti dai contratti collettivi stipulati dalle organizzazioni dei lavoratori e dei datori di lavoro comparativamente più rappresentative.

58 Sul tema v. il fasc. n. 4/2019 della rivista Variaz. Temi Dir. Lav.

59 D. Garofalo, Il lavoro nel terzo settore, in Mass. Giur. Lav., 2018, p. 113: la disciplina de qua non si potrebbe, cioè, considerare una nuova tipizzazione per via legislativa dell'art. 36 Cost., bensì un obbligo derivante direttamente dalla «struttura giuridica conseguente all'attività svolta».

60 Cfr. B. De MozzI, Terzo settore e contrattazione collettiva, cit., pp. 10311035; l'autrice puntualizza che il ragionamento non può valere nei medesimi termini per le cooperative sociali, che sono di diritto imprese sociali, atteso che ciò fa venire meno la logica della norma-incentivo. 
dell'azienda» ${ }^{61}$. Infine, tra le disposizioni del controverso ${ }^{62}$ capo Vbis del d.lgs. n. 81/2015, l'art. 47-quater prevede, per i soggetti richiamati, che (c. 1) «I contratti collettivi stipulati dalle organizzazioni sindacali e datoriali comparativamente più rappresentative a livello nazionale possono definire criteri di determinazione del compenso complessivo che tengano conto delle modalità di svolgimento della prestazione e dell'organizzazione del committente». In mancanza di tali contratti collettivi (c. 2), il compenso minimo orario deve essere «parametrato ai minimi tabellari stabiliti da contratti collettivi nazionali di settori affini o equivalenti sottoscritti dalle organizzazioni sindacali e datoriali comparativamente più rappresentative a livello nazionale».

L'esposizione svolta in questo paragrafo non ha ovviamente pretese di completezza, né vi potrebbe essere la pretesa di approfondire le molteplici e complesse questioni che le normative citate sollevano ${ }^{63}$. Mediante l'individuazione di alcune situazioni particolarmente significative, tale esposizione era volta a preparare il campo alla riflessione vera e propria, che prenderà spunto da questa breve rassegna per continuare ad interrogarsi sul tema della categoria.

61 Cfr. G.A. RecchiA, I rinvii al contratto collettivo nel lavoro agile tra ambiguità normative e ragionevole implementazione dell'istituto, in Arg. Dir. Lav., 2018, p. 1501 ss.

62 La disposizione ha già sollevato in dottrina un ampio dibattito: M. BARBIERI, Della subordinazione dei ciclofattorini, in Lab. Law. Iss., 2019, 2, p. I.1 ss., F. CAPPONI, lavoro tramite piattaforma digitale: prima lettura del d.l. n. 101/2019 convertito in l. n. 128/2019, in Dir. Rel. Ind., 2019, p. 1231 ss., M. Magnani, I tempi e i luoghi del lavoro. L'uniformità non si addice al post-fordismo, in WP CSDLE “Massimo D’Antona”.IT, 404/2019, A. Perulli, La nuova definizione di collaborazione organizzata dal committente, in Riv. It. Dir. Lav., 2019, III, p. 163 ss., G. SANTORo-PaSSarell, Sui lavoratori che operano mediante piattaforme anche digita$l i$, sui riders e il ragionevole equilibrio della Cassazione 1663/2020, in WP CSDLE “Massimo D'Antona”.IT, 411/2020.

${ }_{63}$ Per una trattazione approfondita del tema dei rinvii all'autonomia collettiva, cfr. P. Passalacoua, Autonomia collettiva e mercato del lavoro: la contrattazione gestionale e di rinvio, Torino, Giappichelli, 2005 e, più di recente, I. Alvino, I rinvii legislativi al contratto collettivo, cit. 


\section{Un modello di intervento "leggero" sul sistema sindacale}

A differenza di quanto avviene quando la legge interviene sul sistema di contrattazione collettiva regolandone soggetti, meccanismi di definizione del campo di applicazione e di conclusione degli accordi, regime degli effetti, etc., i rinvii all'autonomia collettiva operano sul sistema contrattuale per così dire dall'esterno ${ }^{64}$. I prodotti dell'autonomia collettiva sono acquisiti ex post, come normatività spontanea emergente dai gruppi sociali ${ }^{65}$. Sono dati, cioè, per presupposti non soltanto l'esistenza, ma anche il corretto funzionamento del sistema di relazioni industriali ${ }^{66}$, ossia la presenza di soggetti rappresentativi dei gruppi di interesse presenti nella società, da una parte, e una condivisa distribuzione di competenze tra tali soggetti, che dia luogo a una ramificazione negoziale nei settori dell'economia, dall'altra parte.

Questo aspetto è stato a lungo considerato uno dei punti di forza del modello italiano. L'idoneità a valorizzare la produzione normativa del sistema di fatto, lasciando a quest'ultimo la prerogativa di darsi in autonomia una propria articolazione ${ }^{67}$, era in qualche modo una quadratura di molti circoli - per riprendere un'espressione di Orsi Battaglini. La cooperazione tra legge e autonomia colletti-

64 Sulla scia della più nota esperienza regolativa dell'età repubblicana: lo statuto dei lavoratori, soprattutto nella sua formulazione originaria, tracciava alcune essenziali situazioni giuridiche di sostegno all'attività sindacale in azienda, dando per presupposta, «non la conformità del sindacato a schemi giuridici prefissati a fini di controllo, ma l'effettiva sua rappresentatività nei confronti dei lavoratori misurata in base a criteri storico-istituzionali» (così, T. TREU, Il sindacato fuori dalla Costituzione: riconoscimento e rappresentatività, in Jus, 1975, p. 216). Cfr., per un assunto largamente condiviso in dottrina, G.F. MANCINI, Lo statuto dei lavoratori dopo le lotte operaie del 1969 (1970), in ID., Costituzione e movimento operaio, Bologna, Il Mulino, 1976, p. 133 ss., M. D’Antona, Diritto sindacale in trasformazione, in ID. (a cura di), Letture di diritto sindacale, cit., p. I ss., V. BAVARo, Il principio maggioritario nelle relazioni industriali, cit., p. 8.

65 L. Mengoni, La questione del «diritto giusto» nella società post-liberale, in Rel Ind., 1988, 11, p. 25, per la teorizzazione di un diritto neo-istituzionale. Per la lettura di tali svolgimenti con le lenti del diritto riflessivo, v. A. Lo FAro, Teorie autopoietiche e diritto sindacale, in Lav. Dir., 1993, p. 129 ss.

66 M. Rusciano, Il contratto collettivo, cit., p. 74.

67 G. Giugni, Giuridificazione e deregolazione nel diritto del lavoro italiano, in Dir. Lav. Rel. Ind., 1986, p. 316 ss. 
va si fonda su un'ipotesi normativa circolare, che si può riassumere in due considerazioni: primo, la legge rinvia al sistema contrattuale senza regolarne i meccanismi di funzionamento e limitandosi a recepirne i prodotti negoziali; secondo, il buon andamento di tali rinvii dipende in sostanza dagli svolgimenti interni a un sistema contrattuale che si mira al tempo stesso a valorizzare e rafforzare.

Tale meccanismo è concepito per risultare win-win. L'ordinamento giuridico può fare affidamento su discipline collettive particolareggiate, rivolte alla gran parte delle tipologie di attività economica presenti nel mercato (cosa che la legge non potrebbe fare con la necessaria perizia e tempestività), senza dover intraprendere il percorso politicamente impegnativo e rivelatosi inattuabile, se non fallace - nella celebre prolusione di Mancini del 1962 -, di una regolazione del sistema di relazioni industriali. A loro volta, gli attori più accreditati del sistema di relazioni industriali dovrebbero risultare valorizzati da queste dinamiche di sostegno, come autorità salariale e normativa del mercato del lavoro, ma preservando un'ampia sfera di autonomia, soprattutto nella struttura organizzativa e della contrattazione collettiva ${ }^{68}$.

Che questo schema sia stato alterato, nei suoi fondamenti, dalla crescita costante dei rinvii in deroga, è stato osservato da anni ${ }^{69}$, ma non è questo un punto che può essere affrontato ${ }^{70}$. Per il tema che ci occupa, occorre puntualizzare, invece, che i rinvii all'autonomia collettiva non alterano i meccanismi di definizione delle unità negoziali invalsi nel sistema di relazioni industriali. Come di norma, le unità negoziali sono definite per accordo tra i soggetti stipulan$\mathrm{ti}^{\mathrm{71}}$. Non serve di ciò alcuna dimostrazione: sarebbe singolare il con-

68 Un cenno in tal senso, con riferimento all'art. 7, 1. n. 31/2008, da parte di M. BARBIERI, In tema di legittimità costituzionale del rinvio al Ccnl delle organizzazioni più rappresentative nel settore cooperativo per la determinazione della retribuzione proporzionata e sufficiente, cit., p. 499: per l'autore la norma citata rappresenta il «frutto tardo - forse l'ultimo - di una stagione legislativa promozionale delle funzioni e del ruolo delle organizzazioni sindacali».

69 A. Perulli, I rinvii all'autonomia collettiva: mercato del lavoro e trasferimento d'azienda, in Dir. Lav. Rel. Ind., 1992, p. 533 ss.

70 Per una recente trattazione, v. A. Pizzoferrato, L'autonomia collettiva nel nuovo diritto del lavoro, in Dir. Lav. Rel. Ind., 2015, p. 411 ss.

71 P. Ichino, Note in materia di estensione dell'efficacia del contratto collettivo, in Riv. Giur. Lav., 1978, II, p. 933. 
trario, che una regolazione «leggera ${ }^{72}$ della contrattazione collettiva possa alterare meccanismi di definizione delle aree negoziali che rappresentano uno dei più decisivi momenti di espressione della libertà sindacale garantita dalla Costituzione. Il «riconoscimento legale» - nelle parole di D’Antona - non interferisce «ex ante sul processo negoziale», ma si limita ad attribuire, «alla contrattazione collettiva come sistema sociale dato», una determinata «qualità normativa nel sistema delle fonti statuali» ${ }^{73}$. Il riconoscimento legale dei contratti collettivi non altera, dunque, le determinazioni congiunte delle parti circa il campo di applicazione. Al proposito, riprendendo una risalente affermazione della Corte costituzionale, può dirsi che in tali situazioni acquisiscono rilievo nell'ordinamento giuridico le categorie che «si sono spontaneamente definite mediante l'autonomo ordinamento sindacale e mediante la stipulazione di contratti collettivi», non altre «sulla base di un'astratta identificazione di attività merceologiche o sulla scorta di altri criteri arbitrariamente prestabiliti $\rangle^{74}$.

3. Le clausole sul campo di applicazione dei contratti collettivi: problemi interpretativi

Per effetto dei rinvii i contratti collettivi acquisiscono riconoscimento legale nel campo di applicazione da essi previsto ${ }^{75}$. In tale ambito, cioè, essi divengono il parametro di misura dei livelli di trattamento economico e, ove previsto, normativo. Eccezionalmente, qualora non vi sia un contratto collettivo al quale fare riferimento, la giurisprudenza (v. per l'art. 36 Cost.) o la legge (ad esempio, art. 23 , c. 16 , d.lgs. n. 50/2016, art. 3, c. 1, 1. n. 142/2001) fanno riferimento ai livelli di trattamento previsti da un contratto collettivo concluso per tipologie di attività non dissimili da quelle regolande (id est per un settore affine). Vi si potrebbe riferire nei termini

72 P. Pascucci, Giusta retribuzione e contratti di lavoro, oggi, cit., p. 76.

73 M. D’Antona, Il quarto comma dell'art. 39 della Costituzione, oggi, cit., p. 680 .

74 Si riprende una considerazione formulata da C. cost. n. 70/1963, cit., in relazione alla Legge Vigorelli (v. supra cap. I, § 6.1).

75 Cfr. G. Proia, L'inquadramento dei datori di lavoro, cit., pp. 304, 306. 
di un singolare élargissement "all'italiana", ossia di un ampliamento indiretto, per via giurisprudenziale (nel primo caso) o legislativa (negli altri), degli effetti dei contratti collettivi oltre il proprio campo di applicazione.

In ogni caso, per stabilire in quali situazioni di fatto sia obbligatorio il rispetto di certi livelli di trattamento economico e/o normativo, è necessario individuare con esattezza i confini del campo di applicazione dei contratti collettivi. Da ciò si può trarre la conseguenza logica che i rinvii all'autonomia collettiva attribuiscono rilievo legale indiretto non soltanto alle clausole che definiscono il trattamento da assumere a parametro (i minimi tabellari, il complesso di voci della retribuzione, l'intero trattamento normativo, etc.), ma anche alle clausole che definiscono l'unità negoziale. Come avviene per le prime, anche le clausole che definiscono il campo di applicazione potranno essere, in caso di controversia, oggetto di interpretazione in sede giudiziale, per verificare quali siano le tipologie di attività nelle quali sia dovuto un certo trattamento.

Che tale prerogativa spetti al giudice è affermazione che trae conferma dalla giurisprudenza costituzionale in materia di legge Vigorelli. In un caso in cui era controversa l'applicabilità ad un'azienda artigiana di un contratto collettivo recepito in decreto dell'esecutivo, la Corte affermò che «non è necessario che la Corte determini i confini concreti della categoria, i quali devono essere desunti caso per caso dalla stipulazione collettiva e con riferimento alle associazioni stipulanti: compito che, nel caso presente e negli altri identici a questo, è di competenza del giudice ordinario» ${ }^{76}$.

Tale precedente è rilevante per il tema che ci occupa. Benché vi sia differenza tra l'ipotesi in cui i contratti collettivi siano recepiti in atti dell'esecutivo e quella in cui gli stessi acquisiscano rilievo legale indirettamente, per effetto di un rinvio "esterno", in entrambi i casi vi sarà la necessità di definire un'area di obbligatorietà dei trattamenti previsti dai contratti collettivi.

Occorre tuttavia precisare che, in relazione alle clausole contrattuali recepite nei decreti delegati, la giurisprudenza riteneva applicabili i criteri di interpretazione previsti dall'art. 12 disp. prel. c.c.

\footnotetext{
76 C. cost. n. 70/1963, cit.
} 
per l'interpretazione della legge ${ }^{77}$. Nelle situazioni di cui ci si occupa non vi è invece motivo di scostarsi dall'orientamento consolidato, sia in giurisprudenza sia in dottrina, secondo il quale le clausole dei contratti collettivi vanno interpretate in base ai criteri previsti dagli artt. 1362 ss. c.c. ${ }^{78}$.

Peraltro, dopo la riforma del giudizio di cassazione (d.lgs. n. 40/2006), tale prerogativa può essere svolta, in via diretta, non soltanto dai giudici del merito - con un controllo indiretto della Cassazione, per via della violazione dei criteri ex artt. 1362 c.c., oppure del vizio di motivazione ex art. 360, n. 5, c.p.c., come avveniva in passato -, ma dalla stessa Corte di legittimità ${ }^{79}$.

Nel complesso, non sembra che l'attribuzione di tale prerogativa al giudice possa essere considerata in sé in violazione del principio di libertà sindacale ${ }^{80}$. In questi casi il giudice non ha modo di influire sulla definizione delle unità negoziali della contrattazione collettiva. Il giudizio non si sofferma sulla validità rispetto alla legge delle clausole che determinano il campo di applicazione dei contratti collettivi, ma si limita a chiarirne il significato, in relazione al caso oggetto della controversia, allo scopo di definire esattamente le tipologie di attività economica nelle quali i contratti collettivi assumono rilievo legale. Né si ravvisa, d'altro canto, alcuna violazione della libertà delle parti dei rapporti di lavoro di stabilire in autonomia quale sia l'associazione alla quale aderire ed eventualmente il contratto collettivo da applicare, purché siano rispettati i minimi di

77 Cfr. E. Gragnoli, Art. 39, in M. Grandi, G. Pera, Commentario breve alle leggi sul lavoro, Padova, Cedam, 1996, pp. 96-97, anche per gli orientamenti espressi dalla dottrina.

78 Sul tema dell'interpretazione dei contratti collettivi ci si limita, senza pretese selettive, a rinviare a E. Gragnol, Profili dell'interpretazione dei contratti collettivi, Milano, Giuffrè, 2000, A. Bollani, Contratto collettivo e interpretazione, Padova, Cedam, 2004, P. Curzio, Nomofilachia e autonomia collettiva, in Dir. Lav. Rel. Ind., 2014, p. 753 ss.

79 Cfr. S. D’Ascola, Controllo del contratto collettivo in Cassazione: spigolature del nuovo orientamento, in Arg. Dir. Lav., 2016, p. 912 ss.

80 Sembra invece di trarre uno spunto critico in tal senso da parte di L. IMBERTI, Trattamento economico minimo (del socio lavoratore) e c.c.n.l. parametro: chi individua la categoria ed il perimetro della stessa?, cit., p. 414, e, se ben s'intende, tempo addietro, in V. Leccese, V. PINTo, Osservazioni in tema di lavoro temporaneo, in E. GHERA (a cura di), Occupazione e flessibilità. Legge n. 196/1997 e provvedimenti attuativi, Napoli, Jovene, 1998, p. 82. 
trattamento previsti dal contratto collettivo dotato di rilevanza legale: diversamente ragionando, si finirebbe per mettere in discussione, più che l'attribuzione al giudice di interpretare le clausole sul campo di applicazione dei contratti collettivi oggetto di rinvio, la complessiva costituzionalità di questa tecnica regolativa, che è invece stata avallata dalla Corte costituzionale ${ }^{81}$.

Tuttavia, è significativo che, per la giurisprudenza che si è occupata del tema, l'interpretazione delle clausole sui perimetri negoziali sia diventata in questi anni il "capo delle tempeste"82. Ciò si deve,

81 Per tutte, C. cost. n. 51/2015, cit.

82 È possibile fornire, tra i molti precedenti in tal senso disponibili, alcuni esempi di sentenze, tratte dalla più recente giurisprudenza ordinaria e amministrativa, che, a fronte di contratti collettivi con ambiti di applicazione similari o parzialmente coincidenti, abbiano operato una selezione tra di essi (o concluso di non poterla operare), sulla base di un'interpretazione delle clausole negoziali. Tali interpretazioni, talvolta, si sono rivelate particolarmente aderenti al dato testuale, mentre delle altre hanno dimostrato una maggiore "libertà" di lettura; è significativo, inoltre, che, nella giurisprudenza di legittimità, ciò sia avvenuto in alcuni casi mediante un mero controllo sull'interpretazione svolta dai giudici del merito, mentre, in altri, proponendo una propria interpretazione delle clausole negoziali.

i) App. Milano 5 luglio 2018, n. 854, a quanto consta inedita, in materia di retribuzione costituzionalmente adeguata ex art. 36 Cost., confronta le aree contrattuali del Ccnl Proprietari di fabbricati (applicato al rapporto di lavoro) e del Ccnl Multiservizi (del quale i lavoratori appellanti chiedevano l'applicazione), respingendo la domanda dei lavoratori sulla base dell'orientamento consolidato ai sensi dell'art. 36 Cost. e dell'art. 2070 c.c., per il quale il contratto collettivo applicato al rapporto di lavoro non può essere messo in discussione sulla base di mere differenze retributive tra i contratti collettivi e di una maggiore "prossimità" tra le attività in concreto svolte e l'area contrattuale del Ccnl del quale si chiede l'applicazione, se non è altresì dedotta l'insufficienza della retribuzione corrisposta in base al contratto applicato al rapporto.

ii) Cass. n. 4951/2019, cit., in materia di soci lavoratori di cooperative, si sofferma sulle clausole che definiscono l'ambito di applicazione del Ccnl Multiservizi e del Ccnl Proprietari di fabbricati, escludendo (non solo per tale argomentazione) l'utilizzabilità quale parametro del secondo $\mathrm{Ccnl}$ «in quanto relativo ad un settore non sovrapponibile a quello dell'appalto»; la Corte afferma inoltre che «la decisione d'appello si fonda su una corretta interpretazione ed applicazione delle disposizioni sopra richiamate e dei contratti collettivi esaminati».

iii) Similmente, Cass. n. 5189/2019, cit., in materia di soci lavoratori di cooperative e appalti pubblici, sugli ambiti di applicazione del Ccnl Multiservizi, del Ccnl Commercio Cisal e del Ccnl Proprietari di fabbricati, e Cass. n. 6143/2019, cit., in materia di appalti pubblici, sulle aree contrattuali del Ccnl Multiservizi e del Ccnl Proprietari di fabbricati.

iv) Cons. St., n. 932/2017, cit., in materia di appalti pubblici, in relazione al «servizio di raccolta e trasporto dei rifiuti urbani e assimilati» nel territorio di un 
da una parte, all'incremento delle disposizioni di legge che rinviano ai contratti collettivi in funzione di parametro del trattamento economico e/o normativo; dall'altra, all'aumento dei casi di sovrapposizione tra gli ambiti di applicazione dei contratti collettivi, che finisce per rendere in qualche misura opinabili gli esiti dell'attività esegetica del giudice e, prima ancora, le scelte delle parti dei rapporti di lavoro. $\mathrm{Al}$ proposito, perciò, che la Cassazione eserciti la funzione nomofilattica sulle clausole che definiscono gli ambiti di applicazione dei contratti collettivi oggetto di rinvio può essere un elemento in grado di fornire certezze agli operatori economici e del diritto circa la selezione dei contratti collettivi da utilizzare quale parametro di trattamento in relazione alle attività di impresa e di lavoro. Lo stesso

ente locale; la sentenza confronta le aree contrattuali del Ccnl Fise Assoambiente e del Ccnl Multiservizi (soffermandosi soprattutto su quest'ultima), convenendo con il giudice del primo grado circa l'applicabilità del Ccnl Multiservizi, che sarebbe coerente (v. art. 1, Ccnl) con l'oggetto dell'appalto; si afferma, in particolare, che: «Il fatto che si faccia espresso riferimento alla pulizia "in domicili privati" non appare di per sé incompatibile con l'applicazione del CCNL nel caso in esame sia perché l'attività di raccolta di rifiuti oggetto di gara è effettuata in larga parte porta a porta, ossia presso domicili privati, sia perché le attività oggetto del CCNL Multiservizi sono individuate "a titolo esemplificativo e non esaustivo", così da poter ricomprendere anche attività di analogo contenuto ma svolte in ambiti diversi, come la pulizia anche di aree pubbliche. Nella stessa prospettiva di una consentita lettura estensiva delle previsioni del contratto l'attività di mera raccolta e trasporto dei rifiuti, senza trattamento o gestione degli stessi, appare riconducibile al novero dei servizi di pulizia ampiamente intesi».

vi) Una lettura estensiva delle clausole sul campo di applicazione del Ccnl Multiservizi è fornita anche da Trib. Milano, 15 giugno 2017, n. 1798, in Pluris, in relazione ad un caso di determinazione della retribuzione di soci lavoratori di cooperative; la sentenza si confronta con l'applicabilità del Ccnl Unci (Confsal, Cisal, Fesica, Cnai) o dei Ccnl (conclusi dalle centrali sindacali storiche) Cooperative di facchinaggio e Multiservizi; quest'ultimo è considerato quello più aderente alle attività svolte dal datore di lavoro convenuto (di confezionamento di salumi) e dalle lavoratrici ricorrenti (di confezionamento e inscatolamento di wurstel).

vii) TAR Milano, Sez. IV, $1^{\circ}$ ottobre 2019, n. 2075, in Pluris, in relazione ad un caso di affidamento in appalto del servizio di gestione delle biglietterie dei Musei civici di Milano, confronta le aree contrattuali del Ccnl Vigilanza non armata - sezione servizi fiduciari, del Ccnl Multiservizi e del Ccnl Terziario, distribuzione e servizi: in particolare, per affermare che il primo di essi non è coerente con l'oggetto dell'appalto, «che attiene all'accoglienza del pubblico in senso lato», mentre il Ccnl è rivolto «alla custodia e sorveglianza dei siti» e, perciò, non è coerente «per ambito tematico, melius per attinenza merceologica e/o teleologica, con l'appalto per la gestione integrata dei servizi di vendita e prevendita di titoli di ingresso, visite guidate, etc.». 
può osservarsi per la giurisprudenza del Consiglio di Stato, in materia di appalti pubblici: data l'entità del contenzioso, non è irrilevante che le amministrazioni, in fase di progettazione degli appalti e, soprattutto, di selezione delle offerte, dispongano di un quadro chiaro in relazione a quali contratti collettivi siano considerati più adeguati rispetto alle tipologie di attività. Ciò non toglie, tuttavia, che sarebbe opportuna una complessiva rivisitazione dei perimetri della contrattazione collettiva di settore, che chiarisca e renda, per così dire, meno esposta a soggettivismi l'individuazione dei contratti collettivi da utilizzare quale parametro del trattamento nei casi di rinvio legale ${ }^{83}$.

4. La selezione dei contratti-parametro: il problema di un ambito residuo di vigenza dell'art. 2070 c.c.

Tanto chiarito, si pone il problema dei criteri da utilizzare per selezionare i contratti collettivi oggetto di rinvio. Il ragionamento non può che cominciare dal considerare che non sempre si rinvengono specifiche indicazioni normative su come effettuare una tale selezione. La considerazione è scontata, se non tautologica, in relazione all'orientamento giurisprudenziale sull'art. 36 Cost., la cui "fisionomia" è il risultato di decenni di riflessione teorica e di evoluzione del diritto vivente ${ }^{84}$. Il valore paradigmatico di tale orientamento, che il legislatore ha tipizzato per una serie via via crescente di segmenti del mercato del lavoro, suggerisce comunque di prenderlo a riferimento. In sintesi, la giurisprudenza afferma che, in caso di nullità per contrasto con l'art. 36 Cost. delle clausole sulla retribuzione, il giudice farà riferimento alle disposizioni del contratto collettivo stipulato per il settore di attività nel quale opera il datore di lavoro, oppure (in mancanza di un contrato collettivo "specifico") a quello di un settore affine.

83 I. Alvino, L. Imberti, Contratto collettivo leader e rappresentanza datoriale, cit., p. 13.

${ }_{84}$ Cfr. G. Zilio Grandi, La retribuzione: fonti, struttura, funzioni, Napoli, Jovene, 1996. 
$\mathrm{Al}$ riguardo, può rimarcarsi come il riferimento al contratto collettivo che includa nel proprio campo di applicazione la tipologia di attività svolta dal datore di lavoro sia una conseguenza dell'organizzazione del sistema di contrattazione collettiva adottata nelle relazioni industriali. Poiché i contratti collettivi definiscono il campo di applicazione per lo più in base alle tipologie di attività svolte dai datori di lavoro, occorrerà fare riferimento a tali determinazioni ${ }^{85}$. Logica vuole che si adoperi un criterio che, da una parte, sia in linea con le previsioni dell'autonomia collettiva e, dall'altra, consenta di selezionare la disciplina collettiva più affine e, perciò, più adatta alle situazioni di lavoro regolande. Si è osservato come ciò sia in linea con le previsioni del cd. Patto per la fabbrica del 2018, con il quale Confindustria e Cgil, Cisl e Uil hanno rimarcato l'esigenza di «una più stretta correlazione tra CCNL applicato e reale attività d'impresa» ${ }^{86}$.

È alla luce di tali considerazioni che andrebbero interpretati anche i rinvii operati dalla legge all'autonomia collettiva. In alcuni casi, è la stessa formulazione letterale delle disposizioni a suggerirlo, come per l'art. 30, c. 4, d.lgs. n. 50/2016, che allude al «contratto collettivo nazionale e territoriale in vigore per il settore e per la zona nella quale si eseguono le prestazioni di lavoro», nonché a «quelli il cui ambito di applicazione sia strettamente connesso con l'attività oggetto dell'appalto o della concessione svolta dall'impresa anche in maniera prevalente»; nel codice degli appalti, anche l'art. 105, c. 9 allude ai «contratti collettivi nazionale e territoriale in vigore per il settore e per la zona nella quale si eseguono le prestazioni». In materia di società cooperative, l'art. 3, c. 1, 1. n. 142/2001 rinvia ai trattamenti previsti dalla «contrattazione collettiva nazionale del settore o della categoria affine» ${ }^{87}$. Peraltro, anche qualora siano assenti nelle disposizioni di rinvio riferimenti siffatti, non sembra che

85 A. Bellavista, La clausola sociale dell'art. 36 st. lav. e l'art. 2070 c.c., cit., p. 470 (v. anche nota 23 per i riferimenti dottrinali).

86 T. TREU, Regole e procedure nelle relazioni industriali: retaggi storici e criticità da affrontare, cit., pp. 14-15.

87 Peraltro, la dottrina ha condivisibilmente puntualizzato come il rinvio debba considerarsi rivolto in primis ai contratti collettivi del settore cooperativo e, solo in seconda battuta, ai contratti collettivi stipulati per le imprese lucrative: cfr. M. Tremolada, Lavoro nelle cooperative, cit., p. 637. 
la logica del ragionamento debba cambiare: ad esempio, in relazione alle discipline in materia di terzo settore e di impresa sociale, che rinviano ai trattamenti previsti dai contratti collettivi di cui all'art. 51 , d.lgs. n. 81/2015, occorrerà considerare la tipologia di attività esercitata $^{88}$.

Tali svolgimenti inducono ad affrontare la questione dell'eventuale vigenza dell'art. 2070 c.c. nell'ordinamento giuridico, atteso che il criterio di selezione dei contratti collettivi basato sull'attività svolta dai datori di lavoro è in sostanza analogo a quello posto dalla disposizione codicistica ${ }^{89}$.

Secondo un primo orientamento, un criterio di questo tipo sarebbe ricavabile implicitamente dalle ripartizioni per aree negoziali della contrattazione collettiva ${ }^{90}$, sul presupposto che quella codicistica, dopo l'abrogazione dell'ordinamento corporativo, sia «norma obsolescente e anzi caducata ${ }^{91}$. Occorre attribuire il riconoscimento legale al contratto collettivo che risulti più adeguato alle situazioni da regolare, in base delle determinazioni adottate dalle parti sociali ${ }^{92}$. Una parte della giurisprudenza perviene ad un esito di questo tipo, atteso che, senza richiamare l'art. 2070 c.c., raffronta il tipo di attività svolta dal datore di lavoro (integrandola talvolta con riferimenti alle mansioni svolte dai dipendenti) con gli ambiti di applicazione dei contratti collettivi, al fine di stabilire quale sia l'accordo più adatto ${ }^{93}$. Specialmente per le disposizioni di rinvio contenute negli artt. 30, c. 4 e 105, c. 9, d.lgs. n. 50/2016,

88 Cfr. B. De Mozzi, Terzo settore e contrattazione collettiva, cit., p. 1028.

89 A. Vallebona, Veri e falsi limiti alla libertà del datore di scegliere il contratto collettivo, in Riv. It. Dir. Lav., 1990, II, pp. 62-63. Più di recente, un cenno in tal senso da parte di P. Tosi, Concorrenza, lavoro, diritti (aspetti collettivo-sindacali), cit., p. 1349.

90 R. Scognamiglio, Il problema dell'applicabilità dell'art. 2070 c.c.: categoria e contratto collettivo, cit., pp. 578-580.

91 M. Pedrazzoli, Qualificazioni dell'autonomia collettiva e procedimento applicativo del giudice, cit., p. 552.

92 Mi pare in tal senso A. Bellavista, La clausola sociale dell'art. 36 st. lav. e l'art. 2070 c.c., cit.

93 V., in materia di retribuzione dei soci lavoratori di cooperativa, Cass. n. 17583/2014, cit., Cass. n. 4951/2019, cit., Cass. n. 5189/2019, cit., Cass. n. 6143/2019, cit., Cass. n. 28289/2019, cit.; nella giurisprudenza di merito, v. App. Milano 17 maggio 2019, n. 720, a quanto consta inedita. In materia di contratti pubblici, v. ad esempio Cons. St., sez. V, 12 maggio 2016, n. 1901, in Pluris, Cons. 
e nell'art. 3, c. 1, 1. n. 142/2001, parrebbe invero superfluo il richiamo all'art. 2070 c.c., posto che la legge sottintende chiaramente che il contratto collettivo oggetto di rinvio debba essere quello "del" settore, ossia quello con il campo di applicazione più confacente rispetto alla tipologia di attività svolta.

Per un secondo orientamento, nelle situazioni descritte, l'utilizzo come parametro del contratto collettivo proprio del settore di attività del datore di lavoro deriverebbe dall'applicazione dell'art. 2070 c.c. ${ }^{94}$. Alcune considerazioni sorreggono l'attribuzione di un ambito residuo o surrettizio di vigenza alla disposizione codicistica $^{95}$. In primo luogo, la norma non sarebbe legata a una concezione autoritaria dei rapporti collettivi, bensì all'instaurazione di un regime di contrattazione collettiva con effetti obbligatori. Essa non sarebbe, perciò, stata abrogata con la caduta dell'ordinamento corporativo, ma semmai resa temporaneamente inutilizzabile: la norma codicistica altro non sarebbe, infatti, se non uno strumento tecnico che, in presenza di un obbligo di applicare i contratti collettivi, consentirebbe di selezionarne uno soltanto, sulla base dell'attività svolta dal datore di lavoro ${ }^{96}$. Non vi sarebbe motivo di

St., sez. V, 5 ottobre 2016, n. 4109, in Giorn. Dir. Amm., 2017, p. 84, nota G. SpaNicciati, Cons. St. n. 932/2017, cit.

94 Tra questi, cfr. G. Proia, L'inquadramento dei datori di lavoro, cit., p. 99 ss., A. VIscomi, Soggettività sindacale e categorie contrattuali, cit., p. 75, T. TREU, Regole e procedure nelle relazioni industriali: retaggi storici e criticità da affrontare, cit., p. 14 (in generale, per le disposizioni che attribuiscono rilevanza legale ai contratti collettivi), P. BellocCHI, Art. 2070, cit., p. 667 (la quale vi allude, in particolare, con riferimento alle discipline sui benefici pubblici alle imprese, sui contratti pubblici e sul minimale contributivo), P.G. Alleva, I profili giuslavoristici della nuova disciplina del socio lavoratore di cooperativa, in Riv. Giur. Lav., 2001, I, p. 373, M. BARBIERI, Cinque anni dopo: il rapporto di lavoro del socio di cooperativa tra modifiche legislative, dottrina e giurisprudenza, in P. Curzio (a cura di), Lavoro e diritti a tre anni dalla legge 30/2003, Bari, Cacucci, 2005, p. 558 (entrambi in materia di discipline dei soci lavoratori di cooperativa), D. Izzi, Lavoro negli appalti e dumping salariale, cit., p. 108 (in materia di appalti pubblici).

95 Si riprende un'espressione di P. Tullini, L'ambito residuo (o surrettizio) di applicazione dell'art. 2070 c.c., in Riv. It. Dir. lav., 1993, II, p. 692 ss.

96 Infatti, essa fu considerata applicabile dalla giurisprudenza costituzionale ai contratti collettivi recepiti in decreto in base alla legge Vigorelli. In particolare, v. C. cost. n. 105/1969, con la seguente argomentazione: «L'art. 2070 cod. civ. risulta, pertanto, diretto a dirimere razionalmente gli eventuali conflitti tra diverse normative collettive, di cui sia invocata l'applicazione ad un rapporto individuale 
non consentirne l'utilizzo, in relazione ai contratti collettivi di diritto comune, ogni qual volta ciò si renda necessario per individuare l'ambito di applicazione delle disposizioni negoziali che fungono da parametro del trattamento minimo.

Questa posizione è stata condivisa dalle Sezioni Unite della Cassazione nella storica sentenza n. 2665/1997 sull'art. 2070 c.c.: dopo averne escluso l'applicabilità ai contratti collettivi di diritto comune, in via di principio, la Corte ha delineato un ambito residuo di vigenza della disposizione codicistica, oltre che per i contratti collettivi corporativi rimasti in vigore e per quelli recepiti nei decreti delegati del $1959^{97}$, per i casi in cui si debba determinare la retribuzione ai sensi dell'art. 36 Cost. e per quelli in cui sia la legge a rinviare ai contratti collettivi. Una parte della giurisprudenza di legittimità segue questo indirizzo in materie quali retribuzione costituzionalmente adeguata ${ }^{98}$, appalti pubbli$\mathrm{ci}^{99}$, fiscalizzazione degli oneri sociali e sgravi contributivi ${ }^{100}$ e minimale contributivo ${ }^{101}$.

Peraltro, quale che sia la tesi da preferire, la sostanza delle cose, adottando l'una o l'altra, non cambierebbe, e la questione potrebbe darsi per risolta, fintantoché il criterio oggettivo di selezio-

di lavoro, e non è in contrasto col principio della libertà ed autonomia sindacale garantito dall'art. 39, primo comma, della Costituzione, in quanto ha riferimento alla nozione di categoria professionale risultante dalla spontanea organizzazione sindacale e dall'autonomia collettiva».

97 Ipotesi di rilievo pratico marginale: v. però le recenti sentenze commentate da E. Gragnoli, L'interpretazione di un contratto collettivo corporativo e il regime previdenziale dei produttori assicurativi, in Dir. Rel. Ind., 2017, p. 814 ss.

98 Cass. 26 gennaio 1993, n. 928, in Riv. It. Dir. Lav., 1993, II, p. 593 ss., nota P. Tullini, Cass. 29 luglio 2000, n. 10002, in Riv. It. Dir. Lav., 2001, II, p. 395 ss., nota E. Manganiello. Una rassegna in C. Galizia, La giusta retribuzione tra punti fermi e questioni aperte, in Dir. Lav. Merc., 2009, pp. 601-605.

99 Cass. 25 luglio 1998, n. 7333, in Riv. It. Dir. Lav., 1999, II, p. 459 ss., nota A. Bellavista.

100 Cass. 26 agosto 2002, n. 12530, in Giust. Civ. Mass., 2002, p. 1581, nota L. Panaiotti, Cass. 4 settembre 2003, n. 12915, in Riv. It. Dir. Lav., 2004, II, p. 319 ss., nota L. Panaiotti, Cass. 16 aprile 2015, n. 7781, in Pluris. V. altresì A. Pizzoferrato, Gli interventi sull'art. 2070 c.c., in C. Zoli (a cura di), Le fonti. Il diritto sindacale, cit., p. 377.

101 Cass. 20 gennaio 2012, n. 801, in Lav. Giur., 2012, p. 407 s., nota G. LuDOVICO. 
ne dei contratti collettivi sia basato sull'attività svolta dal datore di lavoro ${ }^{102}$.

5. Pluralità di contratti collettivi con ambito di applicazione sovrapposto. L'esigenza di dotarsi di più adeguati criteri selettivi

Sono state illustrate altrove in questa ricerca (v. supra, cap. II) alcune delle più evidenti manifestazioni di pluralismo contrattuale nell'attuale sistema di relazioni industriali. Ci si limiterà, perciò, a richiamare le due tendenze di fondo riscontrate.

In primo luogo, l'attenzione è stata posta sulla esistenza di un numero elevato di contratti collettivi conclusi da soggetti diversi da quelli tradizionalmente protagonisti del sistema negoziale (la formula più comune è quella di contrattazione "pirata"). Il senso di tali operazioni è di offrire un'alternativa ai contratti collettivi del circuito della rappresentanza confederale storica, ai fini della regolazione sia dei rapporti di lavoro sia dei sistemi di relazioni sindacali (inclusi enti bilaterali, organismi per la formazione, etc.). Per fare ciò, questi contratti collettivi modulano le proprie unità negoziali sulle tipologie di attività che ambiscono a regolare. Tuttavia, dato che i contratti collettivi del sistema confederale coprono, in sostanza, l'intero arco dei processi economici svolti nel Paese, è inevitabile che i perimetri dei due sistemi negoziali si sovrappongano.

In secondo luogo, si è visto come anche all'interno del sistema contrattuale delle organizzazioni confederali storiche dei lavoratori e dei datori di lavoro si verifichino con frequenza (invero, più di quanto non si pensi) dinamiche di concorrenza regolativa tra i contratti collettivi di settore. Tali dinamiche si manifestano anch'esse mediante la sovrapposizione delle unità negoziali, tra contratti collettivi volti, in via di principio, a rappresentare segmenti del tessuto produttivo e gruppi professionali diversi.

Nel complesso, il risultato è un cospicuo ampliamento della varietà di contratti collettivi che possono essere applicati ai rapporti

102 In tal senso, se ben s'intende, G. OrLandini, Clausole sociali (dir. lav. interno), in Treccani - diritto on line, 2015, § 2.2. 
di lavoro, sia pure mantenendo una coerenza di fondo tra le attività svolte dalle imprese e quelle ricomprese negli ambiti di applicazione dei contratti collettivi ${ }^{103}$. In presenza di una pluralità di contratti collettivi con ambito di applicazione sovrapposto, il criterio oggettivo basato sull'attività svolta dai datori di lavoro finirebbe dunque per rinviare in modo indistinto a ogni contratto collettivo che includa nelle proprie elencazioni di apertura una data tipologia di attività ${ }^{104}$.

Nel linguaggio normativo a queste situazioni si allude con espressioni quali pluralità di contratti collettivi «intervenuti per la ${ }^{105} \mathrm{O}$ «della ${ }^{106}$ medesima categoria. Tali formule sono adottate, di rimando, dall'autorità amministrativa ${ }^{107}$, dalla giurisprudenza ${ }^{108}$ e dalla dottrina ${ }^{109}$. Esse hanno una buona proprietà descrittiva degli svolgimenti in atto nel modello italiano e rappresentano, in un lessico pragmatico adatto alle relazioni industriali, il fenomeno di una molteplicità di coalizioni negoziali - e di contratti collettivi - che si affermano competenti e si fanno concorrenza per un certo ambito della vita economica.

Eppure, a una seconda riflessione queste formule si rivelano inadatte ad afferrare l'essenza del fenomeno. Intese letteralmente, esse non possono avere altro significato se non quello di una varietà di

103 M. BARBIERI, In tema di legittimità costituzionale del rinvio al Ccnl delle organizzazioni più rappresentative nel settore cooperativo per la determinazione della retribuzione proporzionata e sufficiente, cit., p. 501.

104 Non per caso, si è rilevato che il modello di legislazione sindacale basato sul rinvio "esterno" ai contratti collettivi presuppone l'esistenza di «organizzazioni sociali forti, largamente ed effettivamente rappresentative» (L MENGONI, La questione del «diritto giusto» nella società post-liberale, cit., p. 28), che agiscano «in un sistema fondato sulla unicità della categoria contrattuale» (A. GARILLI, Intervento, in Autonomia collettiva e occupazione. Atti del XII Congresso nazionale di diritto del lavoro Milano, 23-25 maggio 1997, cit., pp. 139, 141). Scrive M. Rusciano: i «palliativi escogitati per sopperire alla mancanza dell'erga omnes [...] possono funzionare solo in quanto, nei fatti, esista sempre un unico contratto per ciascuna categoria» (Il contratto collettivo, cit., p. 74).

105 Art. 2, c. 25, 1. n. 549/1995.

106 Art. 7, c. 4, 1. n. 31/2008.

107 V. ad esempio Ministero del Lavoro e delle Politiche sociali, nota 24.3.2015, n. 32/0005623 (in materia di concorrenza di contratti collettivi nel settore delle agenzie assicurative in gestione libera).

108 V. tra le molte Cass. n. 801/2012, cit.

109 A. Lassandari, Pluralità di contratti collettivi nazionali per la medesima categoria, cit. 
contratti collettivi dotati del medesimo ambito di applicazione, ma che prevedono un diverso trattamento economico e normativo; queste formule alludono, cioè, all'esistenza di un'unica “categoria”, nella (o per la) quale siano stipulati una varietà di contratti collettivi. Proprio per tale motivo, esse forniscono una rappresentazione inesatta della realtà, da una parte, e si rivelano, comunque, incompatibili con il concetto di categoria consolidatosi nell'ordinamento giuridico, da un'altra parte ${ }^{110}$.

Dal primo punto di vista, si consideri come siano pochi i casi di contratti collettivi con (esattamente) lo stesso ambito di applicazione, dato che la possibilità di ritagliarsi una sfera di legittimità nel sistema di relazioni industriali deriva proprio dal fatto di ricavare una specifica unità negoziale, anche se solo leggermente diversa da quella dei soggetti concorrenti. La dottrina lo aveva rimarcato quando il fenomeno della contrattazione "pirata" era ai suoi primordi111, ma ora lo dimostrano le analisi (sia pure, work in progress) del Cnel, che ai contratti collettivi depositati nell'archivio dei contratti collettivi associano una serie di codici Ateco ${ }^{112}$, corrispondenti a una o più tipologie di attività ${ }^{113}$.

Dal secondo punto di vista, si può osservare come la giurisprudenza e la dottrina siano da decenni concordi nell'affermare che cia-

110 Non sarebbe, comunque, errato osservare che l'utilizzo della formula di una pluralità di contratti collettivi della (o per la) medesima categoria, più che un vizio genetico frutto di un errore lessicale e concettuale, sia l'esito di un modello di rapporti sindacali e di contrattazione collettiva che, per decenni, è stato in grado di regolarsi ordinatamente, sia sul piano della ripartizione delle aree di influenza organizzativa del sindacalismo dei lavoratori e dei datori di lavoro, sia sul piano delle giurisdizioni negoziali, e per questa via, di pervenire ad un sistema basato sull'unicità dei contratti collettivi nel proprio ambito di applicazione. Si vuol dire, in altri termini, che è più che comprensibile che si alluda, con l'uso di tale espressione, all'esistenza di fatto di "una categoria", tradizionalmente presidiata da una determinata coalizione di organizzazioni sindacali e, da un certo momento in avanti, oggetto di un'invasione da parte di nuove organizzazioni sindacali: occorre, tuttavia, superare questa prospettiva, per le ragioni che saranno illustrate infra nel testo.

111 Cfr. A. Garilli, Intervento, cit., p. 141, A. Lassandari, Intervento, cit., p. 204; più di recente, V. LECCESE, La contrattazione collettiva nazionale oggi: caratteri, metamorfosi e criticità. Temi per il dibattito, cit., p. 114.

112 Istat, Classificazione delle attività economiche Ateco 2007, Roma, Istituto nazionale di statistica, 2009.

113 V., supra, cap. II, § 3.1. 
scun contratto collettivo, per il fatto stesso di essere stipulato per un certo perimetro negoziale, identifichi la "propria" categoria, che si distingue, per definizione, da ogni altra categoria stabilita nel sistema di contrattazione collettiva ${ }^{114}$. La dimostrazione di ciò è fornita, sia pure su un piano descrittivo, sempre dall'archivio pubblico del Cnel, nel quale la ricerca dei contratti collettivi avviene su un duplice livello: un primo livello di ricerca si svolge sulla base di 14 macrosettori (Agricoltura, Chimica, Commercio, Meccanici, etc.); un secondo livello si svolge sulla base delle singole categorie identificate dai contratti collettivi (per stare all'esempio più eclatante, nel macrosettore del Commercio risultano registrati 244 contratti collettivi, in base a dati del dicembre 2019). In buona sostanza, ciò equivale ad affermare che nel sistema di contrattazione collettiva non vi sono oltre novecento Ccnl, bensì oltre novecento categorie contrattuali.

Da queste considerazioni si può trarre un'indicazione utile: anziché a una pluralità di contratti collettivi della (o intervenuti per la) medesima categoria, sarebbe più corretto alludere a una pluralità di contratti collettivi con campo di applicazione in parte (più o meno larga) coincidente (o sovrapposto), dal punto di vista delle attività produttive e/o dei gruppi professionali di riferimento ${ }^{115}$. Questa lettura non soltanto è più corretta sul piano lessicale e dei concetti, ma consentirà, altresì, di affrontare il problema in modo più perspicuo (v. infra, § 6).

Peraltro, prima di interrogarsi su quali possano essere i criteri più opportuni per orientare la scelta tra contratti collettivi con ambito di applicazione sovrapposto, giova chiedersi se sia ammissibile, ab imis, l'eventualità di una loro selezione in via interpretativa. Ad operazioni di questo genere sembrerebbe ostare un precedente della Corte costituzionale reso in materia di legge Vigorelli ${ }^{116}$. In particolare, in un caso in cui erano stati recepiti in decreto due contratti collettivi con ambito di applicazione in parte coincidente, la Corte aveva risolto la questione dichiarando l'illegittimità dei relativi d.p.r., per violazione del principio di libertà sindacale, atteso che, in assenza di una legge

114 V. supra, cap. I, § 5 ss., cap. II, § 2.

115 G. Giugni, Diritto sindacale, con la collaborazione di L. Bellardi, P. Curzio, M.G. Garofalo, Bari, Cacucci, 2008, p. 75.

116 C. cost. n. 106/1963, cit. 
attuativa dell'art. 39 Cost., si sarebbe finito per sopprimere autoritativamente la «rappresentanza, rispettivamente, delle imprese e dei lavoratori che esercitano una attività identica a quella oggetto di sopravvenute associazioni sindacali e di sopraggiunti contratti collettivi». Né - osservò la Corte - sarebbe valso richiamare (era un argomento avanzato da una delle difese) una regola di rapporto tra contratto collettivo "generale" (quello dotato di una sfera di applicazione più estesa) e "speciale" (quello dotato di una sfera di applicazione minore), in analogia ai «rapporti che passano tra legge generale e legge particolare», atteso che ciò avrebbe senso soltanto «in un sistema che definisse e predeterminasse autoritativamente le categorie e ne regolasse autoritativamente la rappresentanza».

Tuttavia, se dalla ricostruzione si procede verso il piano, di diritto vigente, dei rinvii operati all'autonomia collettiva, ci si avvede di come l'ostacolo sia superabile, per due ragioni.

La prima è facilmente intuibile: i decreti delegati attribuivano efficacia erga omnes ai contratti collettivi con un meccanismo diverso da quello previsto dall'art. 39 Cost., rendendoli obbligatori per i datori di lavoro e i lavoratori ricompresi nel loro campo di applicazione. I rinvii all'autonomia collettiva della legislazione vigente non raggiungono invece questa profondità di intervento nel sistema sindacale, ma si limitano a porre un parametro del trattamento economico e, quando previsto, normativo, per i soggetti inclusi nel loro campo di applicazione. Si potrebbe obiettare che la differenza è sottile ${ }^{117}$, ma è proprio su questa (sottile) argomentazione che fa leva la giurisprudenza, quando affronta le questioni dell'efficacia soggettiva dei contratti collettivi oggetto di rinvio e della legittimità costituzionale di questa tecnica di normazione. Non vi è, perciò, lesione della sfera di rappresentanza «delle imprese e dei lavoratori che esercitano una attività identica», visto che si rimane liberi di dotarsi di un diverso regolamento negoziale, benché le parti dei rapporti di lavoro siano tenute ad osservare un determinato parametro di trattamento.

Non è questo, peraltro, l'elemento decisivo per superare l'ostacolo costituito dal precedente della Corte costituzionale. Si tenga

117 A. Vallebona, Giochi di parole sull'erga omnes, in Mass. Giur. Lav., 2015, p. 490 ss. 
presente che uno degli argomenti della sentenza n. 106/1963 consisteva nella mancanza, nella legge di delegazione, di un'esortazione al Governo a procedere con la selezione di uno specifico accordo collettivo, in vista del recepimento in decreto, nei casi in cui ve ne fossero una pluralità con campo di applicazione sovrapposto. È quasi superfluo precisare che, mancando il conferimento di una tale prerogativa al Governo, la legge di delega non prevedeva alcun criterio selettivo. Nel complesso, ciò era più che comprensibile, se si tiene presente che casi simili, benché preconizzati dalla dottrina dopo l'emanazione della legge, non erano all'epoca frequenti come lo sono oggi ${ }^{118}$.

La seconda ragione per la quale il problema rappresentato dalla sentenza n. 106/1963 è superabile consiste, allora, nell'esistenza di indicazioni normative inequivocabili sulla necessità che si proceda a una selezione dei contratti collettivi, al fine di individuare quello (o quelli, se più d'uno) cui attribuire riconoscimento legale. Per quanto, in assenza di meccanismi di misurazione effettiva, la sua capacità di operare in concreto sia dubbia, non può essere attribuito altro significato al criterio della rappresentatività comparativamente maggiore $^{119}$ : oltre al dato letterale, la sua genesi lo dimostra. Dalla frecit.

118 G. GiUGNI, La disciplina legislativa del trattamento minimo di categoria,

119 Se la nozione stessa di rappresentatività si qualifica per una funzione selettiva (V. Bavaro, Rappresentanza e rappresentatività sindacale, in Dir. Lav. Merc., 2012, p. 47), questo non può non essere a maggior ragione vero per la nozione di rappresentatività comparativamente maggiore, volta a operare proprio in contesti di pluralità di accordi collettivi in un medesimo ambito di applicazione (F. LIso, Autonomia collettiva e occupazione, cit., pp. 251-252). Per la giurisprudenza, tra le molte: Cons. St. n. 4965/2015, cit., afferma che il concetto si distingue da quello di sindacato maggiormente rappresentativo «in quanto introduce nella dialettica dei rapporti sindacali un criterio di misurazione di carattere selettivo»; Cons. St. 22 gennaio 2019, n. 537, in Pluris, afferma che il concetto può avere la funzione di «selezionare determinati soggetti sindacali» e di «consentire di scegliere quale sia applicabile, fra due o più contratti collettivi già conclusi, che vengano ad insistere sullo stesso ambito territoriale e categoriale»; TAR Lazio, Sez. III bis, 7 agosto 2014, n. 8865, in Arg. Dir. Lav., 2014, p. 1488 ss., nota V. Putrignano, afferma che «l'avverbio "comparativamente" introduce un elemento di confronto degli oggettivi parametri numerici sulla base dei quali deve essere determinato il grado di rappresentatività». TAR Lazio, Sez. I, 8 febbraio 2018, n. 1522, in Dir. Lav. Merc., 2018, p. 349 ss., nota A. Zoppoli, Riv. It. Dir. Lav., 2018, II, p. 727 ss., nota F. S. GiordANO, afferma che la nozione di rappresentatività comparativamente maggiore ha una 
quenza con la quale il criterio è utilizzato dalla legislazione di rinvio, si è ricavata un'indicazione normativa complessiva, volta a far emergere e a giustificare «l'esigenza di distinguere e ritrovare una sorta di gerarchia tra le molteplicità dei contratti collettivi nazionali vigenti, tutti formalmente dotati di pari forza giuridica» ${ }^{120}$. Tale considerazione si concilia, peraltro, con la logica di fondo della politica del diritto neo-istituzionale, per come identificata nel 1988 da Mengoni, di operare una «ristrutturazione del sistema che lo renda meno legato a $[. .$.$] "ragioni artificiali" del diritto e più capace di dare risposte$ positive e selettive ai mutamenti sociali» ${ }^{121}$.

\section{Selezioni e misurazioni nella maggior rappresentatività com- parata}

Il criterio selettivo cui la legge ricorre è quello della rappresentatività comparativamente maggiore delle organizzazioni stipulanti. Giova precisare sin d'ora che, in relazione alle norme sulle quali si soffermerà specialmente l'attenzione (artt. 23, c. 16, 30, c. 4 , d.lgs. n. 50/2016; art. 7, c. 4 , 1. n. 31/2008), tale requisito è richiesto sia agli agenti negoziali di parte lavoratrice sia a quelli di parte datoriale.

Il primo utilizzo di questa formula risale alla metà degli anni Novanta del secolo scorso (art. 2, c. 25, 1. n. 549/1995, in materia di minimale contributivo). Essa fu adoperata ai fini dell'individuazione dei contratti collettivi da assumere quale parametro, nel caso in cui ne fossero stati stipulati una pluralità per una stessa tipologia di attività. La sua genesi è legata al fenomeno allora incipiente della con-

funzione selettiva, «al fine di commisurare il godimento di determinate prerogative alla effettiva capacità rappresentativa delle organizzazioni soggette al giudizio comparativo» (in materia di individuazione delle organizzazioni sindacali cui attribuire un diritto di partecipazione al Consiglio di vigilanza dell'Anpal).

120 S. Ciucciovino, Mettere ordine nella giungla dei ccnl: un'esigenza indifferibile, cit., p. 229.

121 L. Mengoni, La questione del «diritto giusto» nella società post-liberale, cit., pp. 23-24. 
trattazione collettiva "pirata" ${ }^{22}$, del quale si volevano scongiurare le ricadute sulle fonti di finanziamento del sistema previdenziale ${ }^{123}$. Tale elaborazione si rese necessaria, sotto un profilo tecnico, per la graduale perdita di incisività della nozione di maggiore rappresentatività tout court nella giurisprudenza di merito e di legittimità ${ }^{124} \mathrm{e}$, sotto un profilo di politica del diritto, per gli esiti del referendum popolare che, alcuni mesi prima, aveva travolto quella nozione, in precedenza enucleata dall'art. 19 dello statuto dei lavoratori, rendendola, così, meno "spendibile" 125 .

È noto come la nozione di rappresentatività comparativamente maggiore abbia via via soppiantato quella di maggiore rappresentatività tout court, nei casi di rinvio legale ai contratti collettivi e, più in generale, quale canale di collegamento tra ordinamento statuale e sistema di relazioni industriali ${ }^{126}$. Alcuni esempi significativi: ai sensi dell'art. 2, c. 1, lett. ee), d.lgs. n. 81/2008, sono organismi paritetici quelli costituiti «a iniziativa di una o più associazioni dei datori e dei prestatori di lavoro comparativamente più rappresentative sul piano nazionale» ${ }^{127}$; la costituzione di fondi di solidarietà bilaterali ai sensi

122 P. Passalacoua, Il modello del sindacato comparativamente più rappresentativo nell'evoluzione delle relazioni sindacali, in Dir. Rel. Ind., 2014, p. 382.

123 P. Campanella, Rappresentatività sindacale: fattispecie ed effetti, cit., p. 158.

124 Lo si osservava già da alcuni anni: M. NAPOLI, I sindacati maggiormente rappresentativi: rigorosità del modello legislativo e tendenze della prassi applicativa, in AA.VV., La rappresentatività del sindacato, cit., p. 7 ss. V. altresì L. SILVAGNA, il sindacato comparativamente più rappresentativo, in Dir. Rel. Ind., 1999, p. 212.

125 A. LaSSANDARI, Sulla verifica di rappresentatività delle organizzazioni sindacali datoriali, cit., p. 7.

126 F. CARINCI, Alice non abita più qui (a proposito e a sproposito del "nostro" diritto sindacale), in Dir. Lav. Rel. Ind., 2013, p. 674 ss.

127 Tali organismi paritetici svolgono una serie di rilevantissime prerogative, in una serie di ambiti tra i quali la formazione dei lavoratori: al proposito, si segnala una vicenda occorsa nel settore edile, in relazione al quale il Ministero del lavoro, con lettera circolare 5 giugno 2012, n. 13, aveva fornito chiarimenti al personale ispettivo in merito a quali organismi paritetici potessero considerarsi costituiti da organizzazioni comparativamente più rappresentative. La lettera individuava, «al momento» (cioè, con valutazione aperta ad ulteriori verifiche) come contratti collettivi: a) nell'industria, quello sottoscritto da Ance, Feneal-Uil, Filca-Cisl, Fillea-Cgil; $b$ ) nell'artigianato, quello sottoscritto da Anaepa, Cna-costruzioni, Fiae-casartigiani, Claai, Feneal-Uil, Filca-Cisl, Fillea-Cgil; c) nel settore cooperativo, quello sottoscritto da Ancpl-Legacoop, Federlavoro/Federservizi- 
dell'art. 26, c. 1, d.lgs. n. 148/2015 è riservata agli accordi stipulati «tra organizzazioni sindacali e imprenditoriali comparativamente più rappresentative a livello nazionale»; il consiglio di vigilanza dell'Anpal è composto, ai sensi dell'art. 6, c. 4, d.lgs. n. 150/2015, da dieci «esperti [...] designati dalle associazioni dei datori di lavoro e dei lavoratori dipendenti comparativamente più rappresentative sul piano nazionale» ${ }^{128}$.

In questa sede interessa soffermarsi sui casi di rinvio ai contratti collettivi ai fini della definizione di un parametro del trattamento economico e/o normativo, per affrontare soprattutto due problemi. Il primo consiste nel verificare se sia possibile superare l'orientamento dottrinale secondo il quale non potrebbe effettuarsi una comparazione e, perciò, una selezione tra contratti collettivi, se non in presenza di ambiti di applicazione esattamente sovrapponibili (in-

Confcooperative, Pls-Agci, Feneal-Uil, Filca-Cisl, Fillea-Cgil; $d$ ) per la piccola e media industria, quello sottoscritto da Aniem, Feneal-Uil, Filca-Cisl, Fillea-Cgil. Questa determinazione del Ministero è stata impugnata con ricorso al TAR Lazio da Unci e Confsal. Il Tribunale ha respinto il ricorso (sentenza n. 8765/2015, cit.). Ciò sul presupposto che la circolare si fosse limitata a dare chiarimenti ai servizi ispettivi, che la determinazione fosse temporanea («al momento») e che, comunque, il Ministero (nella memoria depositata) avesse chiarito che, «in assenza di criteri oggettivi normativamente determinati per l'individuazione in termini comparativi della maggiore rappresentatività si è servito» di «dati numerici in continua verifica ed aggiornamento» «periodicamente riveduti e trasmessi dalle medesime OO.SS.», sulla base degli «indici tradizionalmente individuati ai fini del "vecchio criterio" della maggiore rappresentatività e dunque [...] il numero delle imprese associate, dei lavoratori occupati, la diffusione territoriale, la partecipazione effettiva alle relazioni industriali».

128 Tale determinazione è rimessa dalla medesima disposizione a un decreto del Presidente del Consiglio, su proposta del Ministro del lavoro e delle politiche sociali. La prerogativa è stata esercitata con d.p.c.m. del 24 novembre 2016, che ha attribuito i 5 seggi a disposizione delle organizzazioni dei lavoratori, ripartendoli tra Cgil (2 seggi), Cisl (2 seggi) e Uil (1 seggio). La Confsal ha impugnato con ricorso al TAR Lazio il decreto suddetto. Il Tribunale (TAR Lazio n. 1522/2018, cit.) ha respinto il ricorso, con decisione confermata dal Consiglio di Stato (Cons. St. n. 537/2019, cit.). Quest'ultimo, in particolare, ha affermato che la valutazione sulla rappresentatività comparativamente maggiore è stata effettuata dall'amministrazione in modo corretto, sulla base dei dati «più aggiornati all'epoca in proprio possesso» (e perciò con valutazione aperte a futuri capovolgimenti), con riferimento ai seguenti criteri: consistenza numerica degli associati delle singole organizzazioni sindacali, ampiezza e diffusione delle strutture organizzative, partecipazione alla stipulazione e alla formazione dei contratti collettivi nazionali e partecipazione alla trattazione delle controversie individuali e collettive di lavoro. 
fra, § 6.1). Il secondo è determinare quali siano, nel "cantiere aperto" delle relazioni industriali di questi anni, gli indici sintomatici da utilizzare per verificare la rappresentatività (comparativamente maggiore) delle organizzazioni stipulanti (infra, § 6.2). Tali problemi, messi in luce tempo addietro ${ }^{129}$, sono stati a più riprese affrontati dalla letteratura di settore ${ }^{130}$. Tuttavia, solo di recente sembra che i nodi siano venuti al pettine, per effetto, da un lato, dell'incremento dei casi di rinvio all'autonomia collettiva e, dall'altro, della frequenza con la quale si registrano casi di sovrapposizione tra le unità negoziali della contrattazione collettiva.

\subsection{La comparazione tra contratti collettivi con ambito di appli- cazione esattamente coincidente}

Il primo problema da affrontare è quello dell'ambito nel quale operare la comparazione. Si tenga presente che le proposizioni normative di cui ci si occupa suggeriscono che il riconoscimento legale debba avvenire in favore dei contratti collettivi stipulati da parte di (o delle) organizzazioni più rappresentative "del" settore o "della" categoria o simili. Ad esempio, l'art. 7, c. 4, 1. n. 31/2008 allude ai trattamenti previsti dalle organizzazioni più rappresentative a livello nazionale «nella categoria»; l'art. 30, c. 4, d.lgs. n. 50/2016 si riferisce al contratto collettivo «in vigore per il settore e per la zona», purché sia stipulato dalle organizzazioni più rappresentative sul piano nazionale; in altri casi (v. artt. 13, d.lgs. n. 112/2017 e 16, d.lgs. n. 117/2017), la legge si limita a rimandare ai contratti collettivi di cui all'art. 51, d.lgs.

129 Anche prima che fosse introdotta la nozione di rappresentatività comparativamente maggiore: $\mathrm{cfr}$. B. VENEZIANI, Il sindacato dalla rappresentanza alla rappresentatività, cit., p. 401, ma passim. Dopo la sua introduzione, v. M. Roccella, Contrattazione collettiva, azione sindacale, problemi di regolazione del mercato del lavoro, cit., p. 355.

130 Cfr. P. Lambertucci, Contratto collettivo, rappresentanza e rappresentatività sindacale: spunti per il dibattito, cit., p. 578, P. BELLocCHI, La libertà sindacale, cit., p. 39, A. LaSSANDARI, Sulla verifica di rappresentatività delle organizzazioni sindacali datoriali, cit., p. 7, V. PAPA, Verso una rappresentanza misurata? Strumenti e metodi di misurazione della rappresentatività datoriale, cit., p. 38, M. Forlivesi, La rappresentatività datoriale: funzioni, modelli, indici di accertamento, cit., p. 527. 
n. 81/2015, ossia ai «contratti collettivi nazionali, territoriali [o aziendali] stipulati da associazioni sindacali comparativamente più rappresentative sul piano nazionale».

Si pone, tuttavia, il dubbio che tali previsioni (o altre di simile tenore) possano effettivamente operare, qualora i contratti collettivi differenzino, in modo più o meno marcato, i propri ambiti di applicazione. In particolare, secondo una certa interpretazione, il fatto stesso che non vi sia "una" categoria nell'ambito della quale effettuare la comparazione renderebbe infattibile l'intera operazione. Il ragionamento è riassumibile in una considerazione: non è possibile stabilire quali siano le organizzazioni (comparativamente) più rappresentative, se il bacino nel quale verificare la loro rappresentatività non è esattamente il medesimo. È anche a questo proposito che, nel 1997, all'indomani della sua prima introduzione nel diritto positivo, Giugni definiva il criterio della maggiore rappresentatività comparata una «escogitazione linguistica intelligente e feconda, ma che apre il problema più che risolverlo» ${ }^{131}$.

L'argomento secondo il quale comparazione può esservi soltanto se l'ambito nel quale effettuarla sia omogeneo si fonda su una logica rigorosa, nell'interpretazione delle leggi di rinvio ai contratti collettivi ${ }^{132}$, secondo la quale «il punto debole di questa formula [...] potrebbe essere rappresentato dall'ambito categoriale nel quale questa maggiore rappresentatività andrebbe misurata» ${ }^{133}$ : ogni volta che sia creata «una nuova categoria», il sindacato «stipulante sarebbe organizzazione più rappresentativa di quella categoria» ${ }^{134}$. Qualora, cioè, «si distingua - sia pure in modo infinitesimale - ma comunque si distingua dalla categoria del contratto stipulato dal soggetto [...] tradizionalmente presente» ${ }^{135}$, «la stessa funzione selettiva del crite-

131 G. GIUGNI, Intervento, in Autonomia collettiva e occupazione. Atti del XII Congresso nazionale di diritto del lavoro Milano, 23-25 maggio 1997, cit., p. 241.

132 Cfr. G. Giugni, Diritto sindacale, cit., p. 75.

133 F. Liso, Autonomia collettiva e occupazione, cit., p. 243.

134 A. Garilli, Intervento, in Autonomia collettiva e occupazione. Atti del XII Congresso nazionale di diritto del lavoro Milano, 23-25 maggio 1997, cit., pp. 141-142.

135 A. Lassandari, Intervento, in Autonomia collettiva e occupazione. Atti del XII Congresso nazionale di diritto del lavoro Milano, 23-25 maggio 1997, cit., p. 204. 
rio adottato risulterebbe frustrata ${ }^{136}$. Sarebbe sufficiente, insomma, ritagliare «un ambito di riferimento più ristretto - una sub-categoria», per «sfuggire alle maglie di una comparazione che $[. .$.$] vedreb-$ be perdenti» ${ }^{137}$. Come ogni realtà professionale sarà «maggioritaria o unica rispetto al proprio interesse collettivo» ${ }^{138}$, anche le organizzazioni datoriali (sulle quali è oggi posta per lo più l'attenzione), a condizione di trovare un interlocutore negoziale, potranno delineare su misura «il perimetro di misurazione della propria rappresentatività comparata» ${ }^{139}$. Eventuali «strategie opportunistiche», che nel sistema italiano di relazioni industriali non sono «escluse né sopprimibili ${ }^{140}$, consentiranno di aggirare facilmente le previsioni di legge. Persino i più recenti tentativi di conferire maggiore incisività alla legislazione di rinvio (v. gli accordi interconfederali e le convenzioni con Inps e Inl per la misurazione effettiva della rappresentatività sindacale) sarebbero vani, dato che «in assenza di efficacia soggettiva generalizzata delle aree merceologiche chiunque potrebbe [ad esso] sottrarsi, ritagliandosi un perimetro di rappresentanza e rappresentatività su misura ${ }^{141}$.

Semplificando, secondo questo orientamento dottrinale, la formula legale che valorizza la maggiore rappresentatività comparata "del” settore, "della" categoria e simili si risolverebbe in un rinvio a ciascuna unità contrattuale compatibile con le attività svolte dai datori di lavoro, atteso che ogni coalizione - essendo l'unica - nel proprio ambito di applicazione sarà probabilmente anche la più rappresentativa $^{142}$.

136 V. Leccese, V. Pinto, Osservazioni in tema di lavoro temporaneo, cit., p. 83.

137 P. CAmpanella, Rappresentatività sindacale: fattispecie ed effetti, cit., p. 170.

138 P. Bellocchi, La libertà sindacale, cit., p. 39.

139 M. Forlivesi, La rappresentatività datoriale: funzioni, modelli, indici di accertamento, cit., p. 527.

140 V. PAPA, Verso una rappresentanza misurata? Strumenti e metodi di misurazione della rappresentatività datoriale, cit., p. 38.

141 M. Tiraboschi, E. Massagli, La convenzione con INPS e INL, tra passi avanti e i nodi politici della regolazione della rappresentanza che permangono, in Boll. Adapt, 23 settembre 2019, n. 33.

142 In giurisprudenza si rinvengono alcuni precedenti nei quali è svolta una tale argomentazione: v. ad es. Trib. Roma 29 gennaio 2019, n. 840, a quanto consta inedita, resa in materia di calcolo del minimale contributivo. Come noto, l'art. 2, c. 25 , 1. n. 549/1995 (di interpretazione autentica dell'art. 1, d.l. n. 338/1989, 


\subsubsection{Critica}

Queste argomentazioni sono il frutto, come si diceva, di una logica rigorosa, ma si espongono altresì ad alcuni rilievi critici. Il primo di essi fa leva sulla ratio della legislazione di rinvio. Si è avvertito supra (spec. §5), che il comune denominatore di tali interventi del legislatore è proprio di operare una selezione tra contratti collettivi con ambito di applicazione sovrapposto, in vista del conseguimento di una varietà di obiettivi di politica del diritto, che la giurisprudenza e (sembra di poter dire) la prevalente dottrina considerano coerenti con le direttive costituzionali.

Per non fornire che alcuni elementi di contesto, nelle discipline che rafforzano la garanzia costituzionale di una retribuzione adeguata, come quelle in materia di società cooperative o di enti del terzo settore, viene in rilievo l'art. 36 Cost.: disposizioni come quelle più volte menzionate sono «espressione di un interesse costituzionalmente protetto», atteso che offrono «un criterio per la scelta dei contratti collettivi che forniscano più garanzie ai lavoratori nel determinare la retribuzione sufficiente, "proporzionata alla quanti-

conv. con modifiche in 1 . n. 389/1989) prevede che «in caso di pluralità di contratti collettivi intervenuti per la medesima categoria, la retribuzione da assumere come base per il calcolo dei contributi previdenziali ed assistenziali è quella stabilita dai contratti collettivi stipulati dalle organizzazioni sindacali dei lavoratori e dei datori di lavoro comparativamente più rappresentative nella categoria». Nel caso di specie, in relazione ad attività di servizi svolte in appalto in favore di strutture alberghiere, era controversa l'applicabilità del Ccnl concluso tra Unicoop e Ugl Facility management (applicato dal datore di lavoro) oppure del Ccnl Turismo concluso tra Federalberghi, Faita, Confcommercio e Filcams-Cgil, Fisascat-Cisl, Uiltucs-Uil (a partire dal quale l'Inps aveva calcolato l'ammontare dell'obbligo contributivo). Il primo contratto collettivo ha un ambito di applicazione più ristretto e in qualche modo "tarato" sulla tipologia di attività svolta dal datore di lavoro, mentre il secondo ha un ambito di applicazione più ampio, che mira a ricomprendere una maggiore varietà di attività nel settore alberghiero. L'Inps ne affermava l'applicabilità sul presupposto della rappresentatività comparativamente maggiore delle organizzazioni firmatarie, sia dal lato dei datori di lavoro sia dal lato dei lavoratori. Il Tribunale ha rilevato tuttavia che la verifica di rappresentatività comparativamente maggiore «viene in rilievo solo in caso di pluralità di CCNL stipulati per il medesimo settore», mentre «il CCNL Unicoop e Ugl è l'unico nel settore di riferimento - molto specifico - della società» (si rilevava, altresì, che le organizzazioni contrapposte che lo hanno stipulato sono soggetti rappresentativi a livello nazionale). 
tà e qualità" del lavoro svolto» ${ }^{143}$. Nelle singole ipotesi, peraltro, alla garanzia di livelli retributivi adeguati è possibile ricollegare obiettivi aggiuntivi: ad esempio, la tutela della concorrenza nel mercato tra società cooperative e società lucrative ${ }^{144}$, oppure la qualità dei servizi erogati da parte di enti del terzo settore, in attuazione del principio di sussidiarietà di cui all'art. 118 Cost. $^{145}$. In materia di contratti pubblici opera, altresì, l'art. 97 Cost., che giustifica l'adozione di misure volte al contenimento dei disagi che potrebbero conseguire alla corresponsione di basse retribuzioni da parte delle imprese che effettuano $\mathrm{i}$ lavori o i servizi ${ }^{146}$; non è errato rimarcare come tali discipline abbiano, altresì, il fine non meno importante di contrastare forme insidiose di dumping salariale in grado di alterare il mercato del lavoro ${ }^{147}$, in attuazione dell'art. 35 Cost. ${ }^{148}$. Per le discipline previdenziali, gli artt. 36 e 38 Cost. forniscono una base più che solida per tali attività di razionalizzazione del pluralismo contrattuale ${ }^{149}$.

Ora, qualora si aderisse al ragionamento criticato, portandolo ai suoi naturali esiti, si giungerebbe a conclusioni incompatibili con la logica selettiva delle predette disposizioni di rinvio e con le direttive costituzionali che le sorreggono. In primo luogo, qualsiasi coalizione negoziale, anche se composta da soggetti non accreditati nel tessuto produttivo e tra la forza lavoro, per il fatto stesso di definire, mediante contrattazione collettiva, una specifica unità negoziale, potrebbe "intercettare" i rinvii operati dalla legge. In secondo luogo, nei casi, sempre più frequenti, in cui il pluralismo contrattuale derivi da divisioni interne ai circuiti della rappresentanza confederale dei lavoratori o dei datori di lavoro, l'orientamento criticato condurrebbe ad uno stallo continuo, ta-

143 C. cost. n. 51/2015, cit.

144 Cfr. C. Zoli, E. Cusa, Art. 3 (Premessa), in L. Nogler, M. Tremolada, C. Zoli (a cura di), La riforma della posizione giuridica del socio lavoratore di cooperativa, Nuove Leggi Civ., 2002, p. 407.

145 Cfr. D. Garofalo, Il lavoro nel terzo settore, cit., p. 93.

146 C. cost. n. 226/1998, cit.

147 M.G. Garofalo, Per una teoria giuridica del contratto collettivo. Qualche osservazione di metodo, cit., p. 526.

148 G. PERA, Il rispetto della contrattazione collettiva nelle concessioni di pubblici servizi, in Giust. Civ., 1998, p. 2427.

149 Cass. SSUU 29 luglio 2002, n. 11199, in Giur. It., 2003, p. 1807, nota P. Capurso. V. altresì M. Persiani, Autonomia collettiva e retribuzione imponibile, in Mass. Giur. Lav., 1996, p. 670 ss. 
le da impedire di selezionare il contratto collettivo (o i contratti collettivi, se del caso) al quale (o ai quali) attribuire il riconoscimento legale.

Giova, perciò, provare a ricavare una lettura alternativa a quella indicata, come suggerisce l'argomentazione svolta supra (v. § 5 ma, amplius, essa si ricava invero dai capp. I-II). Il fatto stesso di ragionare in termini di una pluralità di contratti collettivi della - o nella medesima categoria (tra i quali operare la comparazione) è inesatto, nella lettura della realtà negoziale e nella comprensione dei concetti giuridici. Da un lato, ciò non sempre corrisponde alle dinamiche di un sistema di relazioni industriali nel quale ciascuna coalizione negoziale, anche - al limite! - per il semplice utilizzo di un certo lessico, stabilisce un proprio campo di applicazione delle discipline collettive. Dall'altro, secondo un'interpretazione del principio di libertà sindacale consolidata da decenni nella giurisprudenza costituzionale e nella dottrina, la stipulazione di un contratto collettivo ha per effetto di qualificare una categoria che, per definizione, sarà diversa da quelle di altre coalizioni.

Ciò equivale, in sostanza, ad affermare che non vi sono "categorie" nelle quali operare una comparazione, ma soltanto contratti collettivi che, nel creare proprie unità negoziali, potranno essere posti a confronto. Posto che ogni coalizione è libera di stipulare contratti collettivi, di farlo per le unità per le quali le parti sociali si assumono reciprocamente rappresentative e di premere, con gli strumenti del confronto al tavolo e della lotta sindacale, perché il proprio contratto collettivo sia (o divenga) quello di riferimento per una certa tipologia di attività, è possibile ritenere che ciò sia compatibile con la scelta dell'ordinamento statuale di stabilire una prevalenza a fini retributivi e, ove previsto, normativi tra i contratti collettivi ${ }^{150}$.

\subsubsection{Spunti per una ricostruzione alternativa}

Alla luce dei rilievi formulati, sembrerebbe più conciliabile con la legislazione di rinvio, oltre che con il quadro costituzionale in cui questa si inserisce, una lettura alternativa dei rinvii ai contratti col-

150 S. Ciucciovino, Mettere ordine nella giungla dei ccnl: un'esigenza indifferibile, cit., p. 229. 
lettivi stipulati dalle organizzazioni comparativamente più rappresentative "del settore", "della categoria" e simili. Tali richiami potrebbero essere letti come una sollecitazione del legislatore a verificare la sussistenza di un duplice requisito, «anche in presenza di una pluralità di accordi astrattamente operanti nel medesimo ambito economico» ${ }^{151}$.

Da un lato, occorre individuare, tra i contratti collettivi con unità negoziali sovrapposte, quello (o quelli) con il "collegamento" più adeguato, giusta il campo di applicazione, con le attività economiche per le quali vada fissato un parametro del trattamento: è una regola che si potrebbe definire di "coerenza oggettiva" (se si vuole, ancor meglio, di "prossimità") tra la contrattazione collettiva e le situazioni di lavoro. Dall'altro, gli indici di rappresentatività (ad esempio, il numero di lavoratori iscritti o di imprese associate) dovrebbero confermare che, nel proprio campo di applicazione, il contratto collettivo sia espressione di una coalizione composta da agenti particolarmente accreditati. La legittimazione delle organizzazioni stipulanti nell'ambito negoziale nel quale operano dovrebbe essere il presupposto della (ed al tempo stesso completare la) coerenza oggettiva tra le discipline collettive e le situazioni di lavoro regolande.

La proposta ricostruttiva si sostanzia, perciò, in una disarticolazione, dal punto di vista logico, sia pure in un apprezzamento che resta unitario, degli accertamenti relativi alla categoria (dei contratti collettivi) ed alla rappresentatività comparativamente maggiore (degli agenti negoziali): sembra così di poter superare i dubbi formulati nel corso degli anni dalla dottrina. Per dare maggiore consistenza a tale proposta, si forniranno alcuni spunti di riflessione, sulla base delle proposizioni normative, più volte citate, di cui ci si occupa, oltre che della più recente giurisprudenza.

Si prendano anzitutto in considerazione le discipline dei contratti pubblici. L'art. 23, c. 16, d.lgs. n. 50/2016 attribuisce al Ministero del lavoro la prerogativa di determinare, settore per settore, il costo del lavoro, mediante «apposite tabelle» redatte sulla base dei contratti collettivi stipulati tra le organizzazioni dei lavoratori e dei

151 P. TuluinI, Concorrenza ed equità nel mercato europeo: una scommessa difficile (ma necessaria) per il diritto del lavoro, cit., p. 210. 
datori di lavoro comparativamente più rappresentative (e delle normative in materia di previdenza e assistenza). La periodica emanazione dei decreti direttoriali sul costo del lavoro attribuisce rilievo parametrico ai «valori economici definiti dalla contrattazione collettiva nazionale» (art. 23, c. 16, cit.) dei soggetti più rappresentativi, al fine di orientare le determinazioni delle amministrazioni in sede di progettazione delle gare e di selezione delle offerte (art. 97, cit.). Sia pure al costo di una certa approssimazione (dato che sono ammessi scostamenti ${ }^{152}$, purché rigorosamente giustificati ${ }^{153}$ e non eccessi$\mathrm{vi}^{154}$ ), questo meccanismo consente di stabilire i livelli minimi di un trattamento economico da considerarsi adeguato alle diverse tipologie di attività. Nei settori caratterizzati da forte pluralismo vi sarà l'esigenza di esaminare una varietà di contratti collettivi ${ }^{155}$, in altri tradizionalmente più stabili (specie, nella manifattura) ${ }^{156}$ la selezione sarà meno problematica ${ }^{157}$, ove le parti sociali diano spazio alla contrattazione di secondo livello questa sarà presa in considerazione ${ }^{158}$, e via così discorrendo, senza escludere la possibilità di "sentire" direttamente le parti sociali ${ }^{159}$. Selezionando i contratti collettivi

152 Da valutare in sede di verifica di congruità dell'offerta: v., di recente, Cons. St., Sez. V, 2 agosto 2018, n. 4785, Cons. St., Sez. V, 28 gennaio 2019, n. 690, Cons. St., Sez. V, 18 febbraio 2019, n. 1097, Cons. St., Sez. V, 18 febbraio 2019, n. 1099.

153 È la condivisile impostazione "rigida" di Cons. St. n. 2252/2017, cit., per la quale una «differenza del costo del lavoro può essere concretamente giustificata dalle diverse particolari situazioni aziendali e territoriali e dalla capacità organizzativa dell'impresa», con possibilità, perciò, di «tenere conto anche delle possibili economie che le singole imprese possono conseguire», ma pur sempre in un quadro nel quale «l'obbligo di indicare costi di lavoro conformi ai valori economici previsti dalla normativa vigente debba valere ed essere apprezzato con particolare rigore». Nello stesso senso, per la precisazione che lo scostamento debba essere «puntualmente e rigorosamente giustificato», Cons. St., Sez. III, 4 gennaio 2019, n. 90, in Dejure.

154 V., tra le molte, Cons. St., Sez. V, 26 novembre 2018, n. 6689.

155 Ad esempio, v. il d.m. 21 marzo 2016, n. 56, sulla determinazione del costo del lavoro nel settore della vigilanza privata.

156 T. Treu, La questione salariale: legislazione sui minimi e contrattazione collettiva, in WP CSDLE “Massimo D'Antona”.IT, 386/2019, p. 21.

157 Ad esempio, v. il d.d. 12 settembre 2019, n. 56, sulla determinazione del costo del lavoro nel settore dell'industria metalmeccanica.

158 Ad esempio, v. il d.d. 27 marzo 2018, n. 23, sulla determinazione del costo del lavoro nel settore delle imprese edili e affini.

159 Cfr. D. GotTARDI, La contrattazione collettiva tra destrutturazione e riregolazione, cit., p. 901. 
oggettivamente attinenti alle situazioni da regolare, l'autorità amministrativa ha l'opportunità di risolvere "caso per caso", in base ai dati in proprio possesso sulla rappresentatività delle organizzazioni sindacali ${ }^{160}$, le ambiguità derivanti dal pluralismo contrattuale e stabilire, così, quale sia, nel complesso, il contratto collettivo più adeguato a fungere da parametro del trattamento.

Che in materia di contratti pubblici vi sia una regola di correlazione oggettiva tra contrattazione collettiva e tipologie di attività è poi confermato dall'art. 30, c. 4, d.lgs. n. 50/2016, in base al quale l'«ambito di applicazione [è necessario che] sia strettamente connesso con l'attività oggetto dell'appalto o della concessione svolta dall'impresa anche in maniera prevalente» ${ }^{161}$. Benché difficilmente le stazioni appaltanti indicheranno, nella legge di gara, a pena di esclusione, uno specifico contratto collettivo ${ }^{162}$, non sembra possa essere revocato in dubbio che le imprese aggiudicatarie siano tenute ad applicare un contratto collettivo che, al contempo, abbia una stretta attinenza oggettiva con le attività economiche da svolgere (da apprezzarsi in base al campo di applicazione dei contratti collettivi $)^{163}$ e sia stipulato da organizzazioni comparativamente più

160 È questo un punto dolente, benché sia al riguardo istruttiva la vicenda di Cons. St. n. 4699/2015, cit. (applicabile ratione temporis il d.lgs. n. 163/2006), nella quale la Sezione ha acquisito, in via istruttoria, esaustivi chiarimenti dal Dirigente responsabile della Direzione Generale della Tutela delle condizioni di lavoro e delle relazioni industriali del Ministero del lavoro, con riferimento: $i$ ) ai criteri utilizzati per apprezzare la rappresentatività sindacale; $i i)$ alla periodicità con la quale sono aggiornate le tabelle; $\mathrm{iii}$ ) alle ragioni del mancato inserimento fra quelli elencati dal decreto del Ccnl (oggetto della controversia) del 27 giugno 2012 concluso tra Cnai e Ucict con Fismic-Confsal e Filcom-Fismic; $i v$ ) all'eventualità che le organizzazioni firmatarie di questo $\mathrm{Ccnl}$ potessero essere annoverate tra quelle più rappresentative; $v$ ) alle differenze tra i valori medi del costo del lavoro indicato dalle tabelle e quelli ricavabili dal suddetto Ccnl; vi) all'eventualità che fossero in passato state riscontrate anomalie per effetto dell'applicazione di questo Ccnl in applicazione di altri contratti pubblici.

161 Per un quadro esauriente, cfr. D. Izzi, Lavoro negli appalti e dumping salariale, cit., pp. 84 ss., 110 ss.

162 Servirebbe a tal fine «una ferra logica di correlazione tra requisiti da indicare e prestazioni da appaltare», secondo quanto affermato da Cons. St. n. 4109/2016, cit.

163 V. Cons. St. n. 5574/2019, cit., che, nel considerare il Ccnl Vigilanza privata - servizi fiduciari estraneo alle attività oggetto della gara («servizio di gestione integrata delle attività di prenotazione, biglietteria inviti, accreditamenti ed acco- 
rappresentative ${ }^{164}$. Giova precisare che tali requisiti potrebbero essere integrati da più d'un contratto collettivo, in relazione ad una medesima gara, qualora nel circuito confederale vi sia una pluralità di contratti collettivi con ambito di applicazione coerente rispetto alle attività svolte stipulati da organizzazioni rappresentative ${ }^{165}$ : ma questo è un esito che soltanto le parti sociali, rivedendo i perimetri negoziali, possono scongiurare.

Benché il dato testuale delle discipline sui contratti pubblici renda più agevole una ricostruzione nei termini indicati, sembra corretto affermarne la proponibilità anche al di fuori di tale cornice normativa, nei casi in cui i contratti collettivi delle organizzazioni comparativamente più rappresentative siano richiamati dalla legge quale parametro per il trattamento economico ${ }^{166}$ e/o normativo ${ }^{167}$. Dalla giurisprudenza più recente si può trarre qualche conferma dell'op-

glienza»), ravvisa invece un'attinenza sufficiente tra le attività e gli ambiti di applicazione del Ccnl terziario e del Ccnl Multiservizi; v. altresì Cons. St. n. 4109/2016, cit., che ravvisa un'attinenza sufficiente tra le attività oggetto della gara («affidamento servizio di autoespurgo su impianti di depurazioni, reti e impianti fognari») e i Ccnl Fise e Gas e acqua.

164 V. Cons. St., n. 4699/2015, cit. Ciò dovrebbe, peraltro, fugare i dubbi che le discipline in esame, anziché favorire il rispetto integrale della contrattazione collettiva, si risolvano nel mero rispetto dei minimi salariali (cfr. D. GoTTARDI, La contrattazione collettiva tra destrutturazione e ri-regolazione, cit., p. 901).

165 Cons. St. n. 4109/2016, cit., Cons. St., n. 932/2017, cit., Cons. St., Sez. V, 17 gennaio 2018, n. 276, Cons. St., Sez. V, 23 luglio 2018, n. 4443, Cons. St., Sez. V, 6 agosto 2019, n. 5574, Cons. St., Sez. V, 12 settembre 2019, n. 6148, TAR Milano, Sez. IV, n. 2075/2019, cit., tutte in Pluris.

166 È il caso della normativa in materia di società cooperative (spec. art. 3, c. 1, 1. n. 142/2001, art. 7, c. 4, 1. n. 31/2008), che impongono di corrispondere un trattamento economico complessivo parametrato a quello dei contratti nazionali; ma è anche il caso della normativa previdenziale sul minimale contributivo, atteso che, ai sensi dell'art. 2, c. 25,1 . n. 549/1995, di interpretazione autentica dell'art. 1, c. 1, d.1. n. 338/1989, conv. con modifiche 1. n. 389/1989, «la retribuzione da assumere come base per il calcolo dei contributi previdenziali ed assistenziali è quella stabilita dai contratti collettivi stipulati dalle organizzazioni sindacali dei lavoratori e dei datori di lavoro comparativamente più rappresentative nella categoria».

167 Può essere il caso degli artt. 13, d.lgs. n. 112/2017, in materia di impresa sociale e 16, d.lgs. n. 117/2017, in materia di enti del terzo settore, che rimandano al trattamento economico e normativo previsto dai contratti collettivi di cui all'art. 51, d.lgs. n. 81/2015; ma è anche il caso della normativa in materia di benefici normativi e contributivi (spec. art. 1, c. 1175, 1. n. n. 296/2006), che sono legati al «rispetto degli accordi e contratti collettivi nazionali nonché di quelli regionali, territoriali o aziendali, laddove sottoscritti, stipulati dalle organizzazioni sindacali 
portunità di tenere distinti, nei termini descritti, gli accertamenti relativi alla coerenza oggettiva dei contratti collettivi rispetto alle attività per le quali si debba stabilire un parametro, da una parte, ed alla rappresentatività comparativamente maggiore dei soggetti stipulanti, dall'altra.

È condivisibile l'orientamento che, sulla base delle clausole che definiscono l'ambito di applicazione dei contratti collettivi, ne verifica la coerenza oggettiva rispetto alle attività economiche oggetto di controversia. In presenza di una pluralità di accordi con ambito di applicazione sovrapposto, in particolare, occorre fare riferimento al contratto collettivo dotato di un ambito di applicazione più «omogeneo» ${ }^{168}$ rispetto all'attività svolta dal datore di lavoro, anche riferendosi ad un «nesso produttivo» ${ }^{169}$, ravvisabile tra le mansioni dei lavoratori ed il settore in cui opera l'impresa. Questo aspetto del giudizio consiste, cioè, in una verifica di «attinenza» ${ }^{170}$ del contratto collettivo rispetto alle attività svolte, dalla quale ne può derivare l' «utilizzabilità» ${ }^{171}$ come parametro del trattamento, a condizione che la stipulazione sia opera di soggetti comparativamente più rappresentativi ${ }^{172}$, ossia legittimati a stabilire una disciplina per quella tipologia di attività.

Tale impostazione valorizza i rinvii che la legge rivolge all'autonomia collettiva: la selezione del contratto collettivo più adeguato potrebbe essere valutata in giudizio sulla base di una duplice regola,

dei datori di lavoro e dei lavoratori comparativamente più rappresentative sul piano nazionale».

168 Cass. 8 maggio 2019, n. 12166, in Lav. Giur., 2019, p. 844, nota G. LudoVICO, in materia di minimale contributivo. Nella specie, tra il Ccnl Trasporto Cisal e il Ccpl confezionatori della provincia di Cuneo, entrambi richiamati nel regolamento della Cooperativa, la sentenza ha confermato la decisione della fase di merito per la quale andasse preso a riferimento il secondo, sia perché stipulato da soggetti comparativamente più rappresentativi, con previsione di livelli retributivi più alti, sia perché maggiormente «omogeneo rispetto all'attività svolta dai lavoratori».

169 Cass. n. 801/2012, cit., in materia di minimale contributivo; Trib. Milano, n. 1798/2017, cit., in materia di retribuzione dei soci lavoratori di cooperative.

170 Cass. n. 10851/2019, cit., in materia di retribuzione dei soci lavoratori di cooperative.

171 Cass. n. 7047/2019, cit., in materia di retribuzione dei soci lavoratori di cooperative.

172 Cass. n. 4951/2019, cit., Cass. n. 5189/2019, cit., Cass. n. 7047/2019, cit., Cass. n. 10851/2019, cit. 
di coerenza oggettiva con le attività da regolare e di provenienza da una coalizione maggioritaria ${ }^{173}$, composta da attori negoziali particolarmente accreditati ${ }^{174}$. Ciò può consentire alla giurisprudenza di arginare gli effetti negativi di operazioni negoziali poco credibili di individuazione di unità negoziali ritagliate $a d$ hoc con intenti di mero abbassamento del costo del lavoro ${ }^{175}$.

\subsection{La misurazione effettiva della rappresentatività: un cantiere aperto nelle relazioni industriali}

Verificare la rappresentatività comparativamente maggiore delle organizzazioni che stipulano i contratti collettivi di categoria pone quantomeno due problemi. Primo, l'assenza di un sistema di misurazione generale della rappresentatività degli attori negoziali rende in certa misura opinabile qualsiasi determinazione adottata dalla giurisprudenza ${ }^{176}$. Secondo, non è scontato poter riferire i criteri tradi-

${ }_{173}$ F. Liso, Brevi note sul protocollo Confindustria, Cgil, Cisl e Uil del maggio 2013, in Riv. Giur. Lav., 2013, I, p. 848.

174 P. Passalacoua, Il modello del sindacato comparativamente più rappresentativo nell'evoluzione delle relazioni sindacali, cit., p. 383, M. ForLIVESI, La rappresentatività datoriale: funzioni, modelli, indici di accertamento, cit., p. 527.

175 In giurisprudenza, v. Cass. 7781/2015, cit., in materia di benefici fiscali/ contributivi, che ha negato l'utilizzabilità, come parametro per il calcolo del minimale contributivo, del Ccnl Cisal (stipulato per le aziende artigiane che svolgono attività di abbigliamento - lavorazioni conto terzi a façon), da parte di imprese façonniste che svolgono, per conto terzi, attività di confezionamento di capi di abbigliamento, limitata ad alcune fasi della lavorazione. È stato considerato applicabile il Ccnl del (più ampio) settore tessile, concluso dalle federazioni aderenti a Cgil, Cisl e Uil, attesa la rappresentatività comparativamente maggiore delle organizzazioni stipulanti. V. altresì Cass. $1^{\circ}$ ottobre 2015, n. 19639, in Pluris. V. anche P. TOMASSETTI, La nozione di sindacato comparativamente più rappresentativo nel decreto legislativo $n$. 81/2015, spec. pp. 371-373. In un altro caso, all'opposto, la giurisprudenza ha dato prevalenza ad un contratto collettivo stipulato per un ambito di applicazione più ristretto, ma concluso da soggetti più rappresentativi, nel confronto con un altro dotato di un ambito di applicazione più esteso: in particolare, Cass. n. 5189/2019, cit. ha negato l'applicabilità, come parametro per la retribuzione dei soci lavoratori di cooperative, del Ccnl concluso dalla Cisal per l'intero settore terziario, preferendovi il Ccnl Multiservizi, concluso dalle federazioni aderenti a Cgil, Cisl e Uil.

176 Cfr. M. PERSIANI, Le vicende della rappresentanza e rappresentatività sindacali tra legge e contratto collettivo, cit., pp. 545-546, 548. 
zionali elaborati dalla dottrina, dalla giurisprudenza e dall'amministrazione per lo più con riferimento alle strutture confederali ${ }^{177}$, anche alle federazioni di settore ${ }^{178}$.

Il dato della rappresentatività comparativamente maggiore non dovrebbe essere dato per scontato, come se si trattasse di un "fatto notorio", in favore degli attori storicamente più accreditati nel sistema di relazioni industriali ${ }^{179}$ : non certamente nel proces$\mathrm{so}^{180}$, ma sembra che non sia più così neanche nella percezione comune ${ }^{181}$.

Nella attuale fase di "riorganizzazione" delle relazioni industriali italiane, il punto di partenza del ragionamento non può che essere costituito dal sistema che le parti sociali stanno mettendo a punto mediante accordi interconfederali, con il sostegno di istituzioni quali l'Inps, il Cnel e l'Inl. Le parti sociali hanno stabilito, ormai in tutte le principali aree di rappresentanza del sistema di relazioni industriali, quali siano (meglio, saranno) i criteri di verifica della rappresentatività delle organizzazioni che stipulano i Ccnl. In particolare, ciò si può affermare per le aree di rappresentanza di Confindu-

177 Tale elaborazione, come noto, non è priva di addentellati nella legge. Gli artt. 2, 1. n. 902/1977 e 4, c. 5, 1. n. 936/1986, indicano i seguenti criteri: consistenza numerica dei soggetti rappresentati, ampiezza e diffusione delle strutture organizzative, partecipazione alla formazione e stipulazione dei contratti collettivi di lavoro, effettiva partecipazione alla trattazione delle controversie individuali, plurime e collettive di lavoro.

178 Cfr. F. Martelloni, Presenza nel conflitto e rappresentatività nell'interregno del sistema sindacale, in Lav. Dir., 2014, p. 55 ss.

179 M.V. Ballestrero, Diritto sindacale, Torino, Giappichelli, 2014, p. 119.

180 Cass. 20 aprile 1999, n. 3912, in Giust. Civ. Mass., 1999, p. 890. In generale, sulla nozione di "fatto notorio" a fini processuali, Cass. 19 marzo 2014, n. 6299, per la quale tale nozione deve essere intesa «in senso rigoroso, e cioè come fatto acquisito alle conoscenze della collettività con tale grado di certezza da apparire indubitabile ed incontestabile». In giurisprudenza di merito, v., in materia di retribuzione dei soci lavoratori di cooperative, Trib. Ivrea 3 novembre 2014, in Pluris, Trib. Milano 5 maggio 2017, n. 1290, Trib. Milano 27 giugno 2017, n. 1313, entrambe in DeJure; in materia di minimale contributivo, v. Trib. Pavia, 26 febbraio 2019 , cit.

181 M. Carrieri, P. Feltrin, Al bivio. Lavoro, sindacato e rappresentanza nell'Italia d'oggi, cit. 
stria $^{182}$, Confapi ${ }^{183}$, Confcommercio ${ }^{184}$, Confesercenti ${ }^{185}$, Confservi-

182 Dopo l'AI del 28 giugno 2011 e il Protocollo d'intesa del 31 maggio 2013, è stato stipulato il TU sulla rappresentanza del 10 gennaio 2014 tra Confindustria e Cgil, Cisl e Uil. Il TU dedica la sua Parte I alla «misura e certificazione della rappresentanza ai fini della contrattazione collettiva nazionale di categoria», con la quale le parti hanno manifestato la volontà di dotarsi di un sistema effettivo di misurazione. In particolare, la rappresentatività delle organizzazioni dei lavoratori sarà verificata, in riferimento ai perimetri della contrattazione nazionale di area confindustriale, sulla base della ponderazione tra dato associativo e dato elettorale. L'accordo è stato modificato da una successiva intesa del 4 luglio 2017, conclusa tra le medesime parti, allo scopo di trasferire all'Inps le prerogative di raccolta del dato elettorale e di ponderazione di questo con il dato associativo prima attribuite al Cnel. Il sistema è stato completato (per il momento) da due convenzioni stipulate tra le parti sociali e l'Inps, una prima il 16 marzo 2015 e una seconda tra le stesse parti, con l'aggiunta dell'Inl, il 19 settembre 2019. L'Inps a sua volta ha diramato istruzioni operative per l'attività di raccolta del dato associativo, dapprima con Circolare n. 76 del 14 aprile 2015 e, in seguito, con Circolare n. 146 del 6 dicembre 2019.

183 Con l'AI in materia di rappresentanza del 26 luglio 2016 tra Confapi e Cgil, Cisl e Uil, Parte prima «Misura e certificazione della rappresentanza ai fini della contrattazione collettiva nazionale di categoria», si prevede un meccanismo di misurazione della rappresentatività delle organizzazioni sindacali basato sulla ponderazione tra dato associativo e dato elettorale. L'accordo è stato modificato da una successiva intesa del 23 settembre 2019, conclusa tra le medesime parti, allo scopo di trasferire all'Inps le prerogative di raccolta del dato elettorale e di ponderazione di questo con il dato associativo prima attribuite al Cnel. Il sistema è stato integrato dalla convenzione stipulata tra le parti sociali, l'Inps e l'Inl in data 27 settembre 2019 al fine di «definire le attività di raccolta, elaborazione e comunicazione del dato associativo, nonché per l'attività di raccolta del dato elettorale e per la sua ponderazione con il dato associativo».

184 Con l'accordo interconfederale del 26 novembre 2015 tra Confcommercio - Imprese per l'Italia e Cgil, Cisl e Uil, sub A) «contrattazione collettiva nazionale di categoria», punto 2) «Misurazione della rappresentatività», le parti hanno convenuto di procedere verso un sistema di rappresentatività misurata delle organizzazioni dei lavoratori, sulla base dei seguenti criteri: $a$ ) dato associativo, $b$ ) dato elettorale, c) numero di vertenze individuali, plurime e collettive di lavoro rappresentate nel settore di riferimento, nel corso del triennio precedente all'avvio dei negoziati per il rinnovo del CCNL (è fornita un'elencazione di tali tipologie di vertenze), $d$ ) pratiche per la disoccupazione certificabili dall'Inps. L'accordo prevede una serie di passaggi per la messa in atto di tale sistema, tra i quali un regolamento che definirà le «modalità specifiche di rilevazione, certificazione e ponderazione degli elementi di cui ai punti c) e d)»e attribuirà «specifici pesi agli indicatori complessivi di cui alle precedenti lettere $a) ; b) ; c) ; d) »$

185 Un sistema simile, basato sui quattro indicatori del dato associativo, del dato elettorale, delle vertenze individuali, plurime e collettive di lavoro e delle pratiche per la disoccupazione certificabili dall'Inps, è delineato con l'AI sul «modello di relazioni sindacali» del 7 settembre 2017 tra Confesercenti e Cgil, Cisl e Uil, sub 
$\mathrm{zi}^{186}$, della cooperazione ${ }^{187}$ e delle organizzazioni dell'artigianato e delle PMI ${ }^{188}$.

Tuttavia, se per le organizzazioni dei lavoratori sono stati delineati indici di misurazione della rappresentatività sostanzialmente incentrati sull'affiliazione sindacale e sul dato elettorale, con alcune variazioni tra gli accordi interconfederali, per le organizzazioni dei datori di lavoro il percorso è in una fase meno avanzata, nella quale ci si limita a riconoscere la necessità di "chiudere il cerchio" e misurare anche la rappresentatività datoriale ${ }^{189}$. Non si tratta di un pro-

A) «contrattazione collettiva nazionale di categoria», punto 2) «Misurazione della rappresentatività».

186 Facendo seguito ad una serie di altri accordi conclusi tra le stesse parti, il TU sulla rappresentanza del 10 febbraio 2014 tra Confservizi e Cgil, Cisl e Uil dedica la Parte I a «Misura e certificazione della rappresentanza ai fini della contrattazione collettiva nazionale di categoria»: si prevede un meccanismo di misurazione della rappresentatività delle organizzazioni sindacali basato sulla ponderazione tra dato associativo e dato elettorale. Il sistema è integrato dalla convenzione stipulata tra le parti sociali e l'Inps in data 16 marzo 2016, che ha affidato all'Inps le attività di raccolta, elaborazione e comunicazione del dato associativo. V. altresì le istruzioni fornite dalla circ. Inps n. 8 del 19 gennaio 2018 e dal messaggio Inps n. 1823 del 30 aprile 2018.

187 Con l'AI del 28 luglio 2015 tra Agci, Confcooperative, Legacoop e Cgil, Cisl e Uil (che aggiorna e supera l'accordo del 18 settembre 2013), mediante le disposizioni della Parte B («misurazione e certificazione della rappresentanza») le parti delineano i fondamenti di un sistema di misurazione della rappresentatività delle organizzazioni sindacali che sarà basato sulla ponderazione del dato associativo e del dato elettorale.

188 Con l'AI sulla rappresentanza del 23 novembre 2016 tra Confartigianato imprese, CNA, Casartigiani, Claai e Cgil, Cisl e Uil, le parti hanno manifestato la volontà di dotarsi di un sistema di misurazione della rappresentatività delle organizzazioni dei lavoratori. Preso atto delle «specificità che contraddistinguono il comparto [e che] rendono necessario adottare anche criteri e indicatori di misurazione della rappresentanza che tengano conto della conformazione delle imprese del comparto», le parti stabiliscono che il sistema sarà basato sul dato associativo, sul dato elettorale (data la elevata presenza di piccole realtà aziendali, non si può contare su una diffusione delle RSU pari a quella di altri comparti, ma è d'altro canto prevista la figura del delegato di bacino) e su «ulteriori indicatori afferenti al complessivo sistema della bilateralità nazionale e regionale».

$189 \mathrm{Su}$ questo profilo di massima sembra che vi sia un consenso diffuso tra $\mathrm{i}$ principali attori delle relazioni industriali nei vari comparti; l'accordo del 14 gennaio 2016 concluso tra Cgil, Cisl e Uil su «Un moderno sistema di relazioni industriali», 3) «Le regole», afferma che «Un compiuto processo di riforma e misurazione della rappresentanza dovrà necessariamente coinvolgere le stesse associazioni datoriali, per superare condizioni di monopolio o di arbitrio estranee ad un moderno sistema di relazioni industriali». Nell'area confindustriale, il Patto per la fabbrica 
blema secondario, atteso che la proliferazione di contratti collettivi di categoria è riconducibile specialmente all'accentuarsi del pluralismo tra le organizzazioni delle imprese. Una delle questioni irrisolte è che le aree di rappresentanza datoriale non sono prive di intersezioni, specie nei comparti artigiano e della piccola e media industria, oltre che delle aziende di servizi. È significativo invero che in alcuni degli accordi interconfederali citati le parti si riconoscano come soggetti comparativamente più ${ }^{190}$ o maggiormente rappresentati$\mathrm{vi}^{191}$, per ambiti di rappresentanza che tra di loro si sovrappongono; nel Patto per la fabbrica del 2018 Confindustria e Cgil, Cisl e Uil riconoscono che il problema non potrà essere risolto se non con «un percorso condiviso anche con le altre associazioni datoriali per arrivare a un modello di certificazione della rappresentanza datoriale».

Per quanto il percorso sia in fieri, è condivisibile l'opinione di quella parte della dottrina che da alcuni anni sollecita la giurisprudenza a valorizzare gli esiti della sistemazione che le parti sociali

del 9 marzo 2018, sub 4) «Democrazia e misura della rappresentanza» riconosce che il percorso di riforma intrapreso negli ultimi anni «per essere compiuto e pienamente efficace, necessita, quindi, della misurazione della rappresentanza anche di parte datoriale». L'AI del 26 novembre 2015 tra Confcommercio - Imprese per l'Italia e Cgil, Cisl, Uil riconosce tra le premesse la «necessità di determinare criteri di misurazione della rappresentatività delle Associazioni dei Datori di lavoro». Con il successivo AI concluso il 24 novembre 2016 tra le stesse parti, per l'area Confcommercio, si ribadisce l'importanza di addivenire ad un sistema di misurazione della rappresentatività datoriale, «e Confcommercio conferma la propria disponibilità ad individuare idonei indicatori in tal senso». Con il citato accordo di area Confesercenti del 7 settembre 2017 le parti «hanno condiviso l'importanza di addivenire a criteri di misurazione non solo per Organizzazioni sindacali, ma anche per la parte datoriale, e Confesercenti conferma la propria disponibilità ad individuare idonei indicatori in tal senso». V. altresì l'AI del 12 dicembre 2018 sulle linee guida per la riforma delle relazioni industriali tra Agci, Confcooperative, Legacoop e Cgil, Cisl e Uil, sub «Cooperazione e misurazione della rappresentanza», che afferma: le parti «convengono sull'opportunità di individuare criteri per la misurazione della rappresentatività anche in ambito datoriale».

190 V. la premessa all'AI del 26 novembre 2015 tra Confcommercio - Imprese per l'Italia e Cgil, Cisl, Uil, la premessa all'AI del 7 settembre 2017 tra Confesercenti e Cgil, Cisl; la premessa all'AI del 28 luglio 2015 tra Agci, Confcooperative, Legacoop e Cgil, Cisl, Uil.

191 V. la premessa all'AI sulla rappresentanza del 23 novembre 2016 tra Confartigianato imprese, CNA, Casartigiani, Claai e Cgil, Cisl, Uil. 
hanno realizzato con gli accordi conclusi a partire dal $2011^{192}$ allo scopo di far emergere, dalla "giungla" negoziale, gli accordi che più di altri siano espressione di interessi autentici provenienti dalle classi produttrici ${ }^{193}$.

Una vicenda della quale giova dare conto è quella originata da un provvedimento del Ministero del lavoro ${ }^{194}$, con il quale l'autorità amministrativa ha effettuato essa stessa una comparazione degli indici sintomatici della rappresentatività delle organizzazioni attive nel settore cooperativo. Mediante utilizzo della «documentazione in possesso di questa amministrazione» - si legge - è operata una verifica sulla base dei seguenti criteri: $a$ ) numero complessivo delle imprese associate; $b$ ) numero complessivo dei lavoratori occupati; $c$ ) diffusione territoriale (numero di sedi presenti sul territorio ed ambiti settoriali); d) numero dei contratti collettivi nazionali stipulati e vigenti; $e$ ) numero dei verbali di revisione. Esito della verifica è il risultato che, nel settore cooperativo, ai fini del minimale contributivo è da prendere a parametro il contratto collettivo sottoscritto da Agci, Confcooperative, Legacoop con Cgil, Cisl e Uil.

Il provvedimento è stato impugnato da Cisal e Confsal davanti al Tar Lazio ${ }^{195}$. I giudici amministrativi hanno affermato la competenza del Ministero ad adottare un tale provvedimento di indirizzo nei confronti degli ispettori «nel quadro generale in materia di misure urgenti per la lotta all'evasione contributiva» e confermato che la rilevazione è stata svolta correttamente. Rileva, invero, che tale provvedimento ha avuto una certa eco nella giurisprudenza successiva, sia in materia di minimale contributivo, sia in materia di trat-

192 Cfr., senza pretese di esaustività, P. Passalacoua, Il modello del sindacato comparativamente più rappresentativo nell'evoluzione delle relazioni sindacali, cit., p. 388, S. MaINARDI, Le relazioni collettive nel "nuovo" diritto del lavoro, in AA.VV., Legge e contrattazione collettiva nel diritto del lavoro post-statutario. Atti delle giornate di studio di diritto del lavoro. Napoli, 16-17 giugno 2016, cit., p. 222, G. FERraro, Teorie e cronache del diritto sindacale e autorità del punto di vista giuridico, in Arg. Dir. Lav., 2016, pp. 36-37, A. Lassandari, Sulla verifica di rappresentatività delle organizzazioni sindacali datoriali, cit., p. 7.

193 Sembra di poter così interpretare lo spunto di G. Ferraro, Teorie e cronache del diritto sindacale e autorità del punto di vista giuridico, cit., p. 37 .

194 Lett. circ. $1^{\circ}$ giugno 2012, prot. 37/0010310/MA003.A004.

195 TAR Lazio, Sez. III bis, n. 8865/2014, cit. 
tamento retributivo dei soci lavoratori di cooperative ${ }^{196}$. Esso è stato richiamato dalla Corte costituzionale nella sentenza n. 51/2015, che ha ritenuto il rinvio ex art. 7, c. 4, cit. «in linea con [tali] specifici indici sintomatici di rappresentatività sindacale». La giurisprudenza di merito ${ }^{197}$ e di legittimità ${ }^{198}$ sta avallando questa soluzione, confermando la possibilità di richiamare in giudizio la rilevazione

196 Non che da ciò si debba trarre un indebito elevamento dei provvedimenti del Ministero del lavoro nell'ordine delle fonti del diritto obiettivo: è il segno, invece, dell'impatto della incisiva riforma del 2007-2008 (art. 7, c. 4, 1. n. 31/2008) sulla razionalizzazione del pluralismo contrattuale nel settore cooperativo (mi pare di poter così interpretare lo spunto di M. BARBIERI, In tema di legittimità costituzionale del rinvio al Ccnl delle organizzazioni più rappresentative nel settore cooperativo per la determinazione della retribuzione proporzionata e sufficiente, cit., p. 497).

197 V., in materia di minimale contributivo, con aperto richiamo alla citata circolare ministeriale, Trib. Monza 5 aprile 2018, in Pluris, App. Bologna 8 agosto 2016, in Pluris, che afferma: «il giudizio relativo alla comparativa maggior rappresentatività va effettuato con riferimento al settore merceologico specifico, tuttavia, una così grande differenza, a livello generale, soprattutto con riferimento alla consistenza associativa e al numero dei contratti collettivi stipulati, non può essere sottovalutata»; identica affermazione in App. Torino 2 novembre 2017, n. 813, in DeJure, che sostiene inoltre: «Vero è che tale documento contiene i dati numerici aggiornati delle OO.SS. datoriali e dei lavoratori a livello nazionale, cioè non riferiti alla categoria, ma deve evidenziarsi che da esso si ricava che la CONFCOOPERATIVE, la LEGACOOP e l'AGCI associano complessivamente 40.025 cooperative con oltre un milione fra dipendenti, soci e soci lavoratori, laddove l'UNCI associa in tutto 10.280 aziende con 168.561 lavoratori dipendenti e che la CGIL, CISL e UIL associano complessivamente oltre 12 milioni di soggetti, laddove la CONFSAL ha dichiarato di associare 1.807.246 unità: l'evidente, enorme sproporzione di consistenza associativa fra la CONFCOOPERATIVE /LEGACOOP/AGCI e l'UNCI quanto alle organizzazioni datoriali e fra la CGIL/CISL/UIL e la CONFSAL quanto alle oo.ss. dei lavoratori ben fa presumere, in assenza peraltro di dati di segno contrario, che anche nell'ambito del settore cui appartiene l'appellante odierna le oo.ss. CONFCOOPERATIVE/LEGACOOP/AGCI siano organizzazioni maggiormente rappresentative dell'UNCI e le oo.ss. CGIL/CISL/UIL siano maggiormente rappresentative rispetto alla CONFSAL». V., altresì, in materia di retribuzione dei soci lavoratori di cooperative, sempre con un aperto richiamo della circolare ministeriale, Trib. Parma 12 novembre 2015, n. 367, a quanto consta inedita, Trib. Parma 26 novembre 2015, n. 379, a quanto consta inedita, Trib. Novara 15 giugno 2017, Trib. Torino 18 marzo 2019, n. 178, in Pluris, App. Milano 17 maggio 2019, n. 720 (che prende in considerazione come indice di rappresentatività datoriale anche il fatturato).

198 Cass. n. 28289/2019, cit., nel confermare la decisione di merito, richiama la circolare ministeriale e gli indici di rappresentatività da essa utilizzati ed esclude di poter rivedere l'accertamento in fatto sulla rappresentatività compiuto nel giudizio di merito. 
ministeriale. Ciò, di fatto, finisce per "trasferire" la rilevazione compiuta sulla rappresentatività confederale in favore delle federazioni che stipulano i contratti di categoria, come in passato avveniva nei giudizi sulla maggiore rappresentatività tout court ${ }^{199}$.

I meccanismi che consentirebbero di selezionare, col minor grado possibile di incertezza, $i$ contratti collettivi che assumono rilevanza legale sono, in qualche misura, in via di affinamento. Se il percorso avviato dalle parti sociali in tutti i principali comparti dell'economia del Paese è in uno stato di avanzamento impensabile soltanto pochi anni fa, ma ancora in itinere, sia l'autorità amministrativa sia la giurisprudenza stanno adoperando gli strumenti che l'ordinamento positivo annovera già, al fine di minimizzare, per quanto possibile, gli effetti che i fenomeni di dumping contrattuale sono in grado di generare sui livelli retributivi, sulle condizioni di lavoro e, in definitiva, sulla stessa tenuta della concorrenza nel mercato ${ }^{200}$.

6.3. Le rilevazioni Cnel-Inps sull'applicazione dei contratti collettivi alle imprese e ai lavoratori ad esse addetti

Fintantoché che non saranno effettivamente operativi i meccanismi di misurazione della rappresentatività elaborati dalle parti sociali e dall'amministrazione, è comprensibile che dalla giurisprudenza non emergano orientamenti pienamente soddisfacenti.

In questo contesto, uno strumento elaborato dal Cnel e dall'Inps può contribuire a individuare il contratto collettivo più adatto a rappresentare il parametro di riferimento in relazione alle diverse tipologie di attività. Si allude al database dei contratti collettivi che consente di verificare quale sia il bacino di applicazione dei singoli Ccnl. Nel settembre 2019 è stato reso pubblico, per la prima volta, il nu-

199 F. CARINCI, Alice non abita più qui (a proposito e a sproposito del "nostro" diritto sindacale), cit., p. 674, segnalava il rischio che la nozione di rappresentatività comparativamente maggiore non fosse risolutiva proprio perché a differenza della «nozione di maggior rappresentatività» non è «declinata con riguardo alle Confederazioni, che la possedevano in via originaria e la trasmettevano alle Federazioni aderenti in via derivata».

200 F. D’Addio, M. Tiraboschi, Part-time senza vincolo di orario minimo e sindacato comparativamente più rappresentativo, cit. 
mero di imprese e di lavoratori ad esse addetti ai quali sono applicati i contratti collettivi di settore, in modo da poter riscontrare in modo oggettivo la rilevanza che ciascuno di essi ha per gli attori economi$\mathrm{ci}^{201}$, in un'ottica, se si vuole, di effettività ${ }^{202}$. Questo risultato è stato raggiunto dalle due istituzioni, a compimento di un percorso di collaborazione avviato da alcuni anni, mediante l'armonizzazione dei codici numerici e alfanumerici che identificano i contratti collettivi, rispettivamente, nell'archivio pubblico Cnel (finalità di conoscibilità dei testi contrattuali) e in quello Inps (alimentato dalle dichiarazioni Uniemens rese dai datori di lavoro per finalità contributive, con indicazione del contratto collettivo applicato) ${ }^{203}$.

In un sistema di relazioni industriali, come quello italiano, caratterizzato da una tradizionale carenza di informazioni sugli attori del sistema e sulle loro attività negoziali, i dati sul tasso di applicazione "effettivo" dei contratti collettivi nei diversi settori produttivi possono avere ricadute positive sugli orientamenti della giurisprudenza e, in prospettiva, sulla stabilità del sistema contrattuale ${ }^{204}$. Il fatto che, delle centinaia di "nuovi" Ccnl depositati presso l'archivio pubblico dei contratti del Cnel, soltanto alcuni "esistono" e sono effettivamente applicati ai rapporti produttivi non è poi un ele-

201 Il database pubblicato fa riferimento all'applicazione dei Ccnl nel periodo dal $1^{\circ}$ gennaio 2018 al 31 dicembre 2018.

202 F. Avanzi, Dimensione e qualità dei contratti. Spunti e riflessioni dall'analisi Cnel-Inps, in Boll. Adapt, 29 luglio 2019, n. 29.

203 V. il documento diffuso da Cnel e Inps su Individuazione dei contratti da assumere a riferimento per l'applicazione della normativa previdenziale e di finanza pubblica, 17 luglio 2019. In particolare, con riferimento ai singoli Ccnl il database fornisce una serie di informazioni relative a: $i$ ) codice Inps; ii) codice Cnel; iii) settore contrattuale Cnel (sulla base dei macrosettori in cui è suddiviso l'archivio pubblico dei contratti); iv) definizione del campo di applicazione sulla base della tipologia di imprese elencate dai contratti collettivi (al riguardo, sono state adottate le seguenti cinque ripartizioni: IND (industria), PMI, ART (artigiani), COOP (cooperazione), DIRIGENTI); $v$ ) destinatari del Ccnl (con un'indicazione sintetica riassuntiva del campo di applicazione); vi) organizzazioni datoriali di categoria firmatarie; vii) organizzazioni datoriali confederali alle quali eventualmente aderiscano; viii) organizzazioni sindacali firmatarie; $i x$ ) numero di aziende alle quali il Ccnl è applicato (sulla base delle dichiarazioni Uniemens); $x$ ) numero di lavoratori ai quali il Ccnl è applicato (sulla base delle dichiarazioni Uniemens).

204 T. Treu, Un segnale unitario per il futuro, in Il Sole 24Ore, 5 giugno 2019. 
mento di poco conto ${ }^{205}$, anche se la rappresentatività è un dato che deve preesistere alla contrattazione collettiva e che deve riguardare i soggetti stipulanti, non i contratti collettivi. Sarebbe sia un errore logico, dopo la pubblicazione di questa banca dati, iniziare a ragionare in termini di "rappresentatività dei contratti collettivi", sia un rischio, atteso che un dato siffatto di effettività dipende, comunque, non dalle qualità e dalla affidabilità dei soggetti stipulanti, bensì dalle scelte dei datori di lavoro. Non è detto che il contratto collettivo più applicato sia necessariamente quello concluso dai soggetti più rappresentativi in una determinata area dei rapporti economico-produttivi.

Tuttavia, il database quantitativo Cnel-Inps può rappresentare, in questa fase "costituente" delle relazioni industriali, un supporto utile, per verificare quale sia la coalizione negoziale che è riuscita a stipulare il contratto collettivo realmente recepito nei rapporti economico-produttivi e che sia perciò considerato quello più "adeguato" dagli stessi attori economici a rappresentare il parametro di riferimento.

205 R. Schiavo, L'analisi congiunta dei Ccnl tra Cnel e Inps: un segnale d'urgenza di un dato sulla rappresentatività delle organizzazioni sindacali, in Boll. Adapt, 29 luglio 2019, n. 29. 
Conclusioni

Le riflessioni svolte in questa ricerca hanno consentito di identificare, nell'art. 39, c. 1, Cost., il fondamento della cruciale attività sindacale con cui le parti sociali stabiliscono il campo di applicazione dei contratti collettivi. Tre argomentazioni, emerse nel corso della ricerca e che ora si può provare a sintetizzare, contribuiscono a spiegare il perché di una piena valorizzazione del principio di libertà sindacale nel tema de quo.

La prima di esse attiene alla storia della materia: non potrebbe non avere un peso che, nell'ordinamento giuridico italiano, l'unica previsione di portata generale di meccanismi legali e amministrativi di definizione delle unità negoziali dei contratti collettivi sia stata adottata, durante il ventennio, nel quadro di un modello autoritario di rapporti sindacali. In particolare, non potrebbe non avere un peso che, nell'esperienza corporativa, i meccanismi di definizione delle categorie contrattuali fossero il completamento delle disposizioni che fissavano l'ambito di inquadramento delle associazioni riconosciute, in un contesto di repressione del pluralismo sindacale. In altri termini, è naturale che la definizione delle categorie sia stata a lungo percepita, più che come un problema di soluzioni tecniche per la contrattazione collettiva (art. 39, c. 4), «come una questione di tutela del sindacato nei confronti dello Stato, al cui interno dominava il timore della predeterminazione legale ed autoritativa degli interessi rappresentati, che aveva caratterizzato il diritto sindacale 
corporativo» $^{1}$ (art. 39, c. 1). A tale riguardo, è significativo che, dalla breve rassegna di diritto sindacale comparato svolta supra (v., spec., cap. III, § 3.2), sia emerso come (anche) nell'ordinamento spagnolo la definizione del campo di applicazione dei contratti collettivi sia considerata dalla dottrina e dalla giurisprudenza uno dei corollari del principio di libertà sindacale dai quali si coglie la differenza con il modello corporativo sperimentato durante il franchismo.

Secondo: fin dagli anni Cinquanta del secolo scorso, in un orientamento poi rimasto, di lì in avanti, costante, la migliore dottrina ha rimarcato che, anche se inclusa nell'articolato dei contratti collettivi, la definizione del campo di applicazione costituisce espressione di una sfera di "giurisdizione" delle parti sociali all'interno delle relazioni industriali. Se si escludono le situazioni singolari degli accordi "pirata", in cui le unità negoziali sono elaborate ad arte con finalità di dumping, il campo di applicazione dei contratti collettivi dovrebbe sempre essere considerato come il risultato di una presenza più o meno radicata delle organizzazioni stipulanti in un determinato segmento della forza lavoro e del tessuto imprenditoriale. Così, limitando, con un intervento eteronomo, l'autonoma descrizione delle unità contrattuali, si finirebbe giocoforza per incidere sullo status del soggetto, irrigidendone gli spazi di operatività nella realtà socioeconomica. È questo che s'intende quando si osserva che è proprio a partire dalla definizione delle unità negoziali che prende corpo l'intervento sindacale nei processi produttivi ${ }^{2}$.

Lo ha dimostrato decenni addietro la vicenda della contrattazione articolata. La "scoperta" di nuovi ambiti di negoziazione è stata un aspetto di prim'ordine del rinnovamento dei corpi intermedi e del ciclo di lotte sociali che ha portato alla legislazione di sostegno nel $1970^{3}$. Si

1 Così, P. Bellocchi, La libertà sindacale, cit., p. 38. Sul tema più generale della diffidenza dei giuristi del lavoro verso ogni ipotesi di interferenza dei pubblici poteri, come reazione al corporativismo fascista degli anni Venti e Trenta del secolo scorso: G. VARDARo, Contrattazione collettiva e sistema giuridico, Napoli, Jovene, 1984, p. 53 ss.

2 V. C. cost. n. 106/1963, cit., secondo la quale la definizione delle categorie contrattuali «si assume e si ammette conforme alla dinamica delle forze del lavoro ed alla struttura delle imprese».

3 G.F. Mancini, I sindacati: fabbrica e riforme, in Pol. Dir., 1971, p. 211 ss. Di recente, riprende il punto $\mathrm{V}$. BAVARO, Rappresentanza e rappresentatività sindacale, cit., p. 35 . 
pensi, altresì, per un esempio più vicino nel tempo, alla lunga vertenza che ha portato nel 2018 alla stipulazione del Ccnl della Distribuzione Moderna Organizzata. Il nuovo contratto realizza la volontà di un segmento consistente del tessuto produttivo del settore (rappresentato da Federdistribuzione) di rescindere il legame tradizionale con il mondo del commercio-terziario, al fine di creare un'autonoma area negoziale e di dotarsi di uno strumento per competere in un mercato fortemente concorrenziale e internazionalizzato, nel quale le aziende hanno «caratteristiche del tutto specifiche» per «dimensioni, per ampia presenza territoriale anche delle attività indotte, per volumi d'affari realizzato e di risorse occupate» (si trae dalla premessa al Ccnl).

Il terzo argomento è uno sviluppo del secondo. La definizione delle unità negoziali è decisiva nel conferire peso rappresentativo e dunque intensità e compattezza all'azione sindacale, sia per le organizzazioni dei lavoratori sia per quelle dei datori di lavoro. A seconda del campo di azione negoziale in cui si muovono, esse avranno una diversa presa sulla base e perciò una diversa forza contrattuale. Detto diversamente, vi è un nesso stringente tra l'individuazione di una sfera di interesse (collettivo) da proteggere, promuovere e perseguire e le attività con le quali, sia pure in accordo con le controparti, viene definito l'ambito di applicazione dei contratti collettivi.

Ampliare l'unità contrattuale consentirà il più delle volte di sopperire alle deficienze organizzative di alcuni settori ${ }^{4}$. Si è osservato, ad esempio, che la scelta del sindacalismo antifascista della seconda metà degli anni Quaranta e dei primi anni Cinquanta del secolo scorso di concentrarsi sulla contrattazione collettiva di livello interconfederale si dovette non soltanto ad una cultura sindacale centralistica e dirigistica, ma anche all'assenza, nei processi produttivi, della compattezza e della presenza tra i lavoratori necessarie per imporre alle organizzazioni dei datori di lavoro una contrattazione di settore ${ }^{5}$. Peraltro un ampliamento

4 A. Vallebona, Il contratto collettivo di lavoro nei Paesi della Comunità europea, Rimini, Maggioli Editore, 1982, p. 109: «ai livelli più vasti di contrattazione diminuisce il grado di interesse comune con l'inevitabile sacrificio delle minoranze, mentre ai livelli più ristretti si evitano tali problemi, ma diminuisce la forza contrattuale del gruppo di lavoratori».

5 L. CASTelvetri, Gli strumenti e i contenuti delle relazioni industriali nel sistema intersindacale: la contrattazione collettiva, in F. PESCHIERA (a cura di), Sin- 
eccessivo dell'unità negoziale può comportare una diminuzione della intensità dell'azione ed un appiattimento verso il basso delle retribuzioni ${ }^{6}$.

$\mathrm{Al}$ contrario, restringere il campo di applicazione dei contratti collettivi può servire a concentrare, in vista della negoziazione con la controparte, le forze di gruppi tra di loro particolarmente coesi, come nell'esperienza della contrattazione di mestiere, o nella stessa contrattazione aziendale ${ }^{7}$.

Del legame tra estensione delle unità negoziali e coesione organizzativa non vi è, nel nostro ordinamento, migliore dimostrazione di quella che offre la contrattazione collettiva del settore pubblico. Fin dalla legge-quadro del 1983, in dottrina si sottolineava che è «lecito presumere che le confederazioni useranno del potere negoziale ad esse attribuito anche ai fini di ritagliare i comparti che risultino più favorevoli alle proprie organizzazioni di categoria e più conformi alle proprie linee di politica organizzativa interna» ${ }^{8}$.

Riepilogando, l'individuazione del campo di applicazione dei contratti collettivi realizza una posizione giuridica che ha sostanza sia di definizione dei contorni operativi dell'organizzazione sia di attività di contrattazione collettiva ${ }^{9}$ e che non si dubita sia «una delle espressioni fondamentali dell'autonomia sindacale, che in tal modo manifesta la diversità dei tipi e delle dimensioni degli interessi collettivi spontaneamente emergenti» ${ }^{10}$. La storia stessa delle relazio-

dacato, industria e Stato nel dopoguerra: storia delle relazioni industriali in Italia dal 1943 al 1948, Firenze, Le Monnier, 1976, p. 384 ss.

6 Un cenno in U. Romagnoli, Il contratto collettivo, in Dir. Lav. Rel. Ind., 2000, p. 229.

7 Benché per la contrattazione aziendale questo non sia naturalmente sempre il caso ed il discorso sia, peraltro, reso più complesso dalle prassi di contrattazione concessiva: per una riflessione di respiro comparato cfr. C. CARTA, La funzione di sostegno alla competitività degli accordi aziendali peggiorativi. Il contributo dell'analisi comparata con i modelli francese e tedesco, in Variaz. Temi Dir. Lav., 2019, p. 603 ss.

8 A. Orsi Battaglini, Della quadratura di molti circoli, cit., p. 11. Peraltro, non sempre gli esiti dei negoziati sui comparti svolti a livello confederale potranno essere in linea con le aspettative delle federazioni di categoria, come dimostra la vicenda illustrata da R. SALOMONE, Rappresentatività sindacale, ambiti negoziali e procedure nella contrattazione collettiva del pubblico impiego, cit., p. 495 ss.

9 Un cenno in V. LeCCESE, Il diritto sindacale al tempo della crisi. Intervento eteronomo e profili di legittimità costituzionale, cit., p. 501.

10 A. Vallebona, Il contratto collettivo di lavoro nei Paesi della Comunità europea, cit., p. 108. 
ni sindacali «mostra che le controversie "giurisdizionali" sono fra le più difficili da comporre fra le parti, perché toccano questioni di identità e di sopravvivenza delle organizzazioni $\gg^{11}$. Per quanto possano essere considerate «fattore di disordine», tali controversie rappresentano infatti «una manifestazione ineliminabile del principio di libertà sindacale, la cui soppressione coatta - si è scritto - equivale all'atteggiamento di chi rompe il termometro per curare la febbre» ${ }^{12}$.

Se si riconosce, come sembra opportuno, che la definizione del campo di applicazione dei contratti collettivi è attività che si colloca in prossimità del "nucleo duro" del principio di libertà sindacale, non ci si può esimere dal trarne le conseguenze, quando ci si interroghi circa l'intensità con la quale questa situazione giuridica è protetta nella formula di garanzia della Costituzione.

Nell'art. 39, c. 1, Cost. sono tutelati sia il profilo dell'organizzazione (sindacale), sia quello dell'attività (di contrattazione collettiva). Ciò non toglie che si possa scorgere una differenza nell'intensità della tutela accordata «a due distinti oggetti, all'organizzazione e alla attività» ${ }^{13}$. Il primo di essi, specie nelle determinazioni che esprimono l'essere medesimo dell'organizzazione, è protetto in modo rigido da interferenze o intromissioni eteronome (con poche eccezioni, come per le forze armate e di polizia), mentre il secondo non è del tutto preservato da un intervento dei poteri pubblici. Per non considerare che alcuni elementi di contesto: non sussiste in favore della contrattazione collettiva una riserva di legge nella disciplina dei rapporti di lavoro ${ }^{14}$; è ammissibile, sia pure entro limiti stringenti, la posizione di "tetti massimi" e di "vincoli" alla contrattazione collettiva ${ }^{15}$; negli accordi collettivi in materia di sciopero nei servizi pubblici essenziali e nella contrattazione collettiva del lavoro pubblico, gli equilibri tra autonomia sindacale e controllo pubblico

11 T. TREU, Regole e procedure nelle relazioni industriali: retaggi storici e criticità da affrontare, cit., p. 13.

12 M. BARbieri, Problemi costituzionali della contrattazione collettiva nel lavoro pubblico, cit., p. 435.

13 Così, A. Orsi Battaglini, Della quadratura di molti circoli, cit., p. 15.

14 C. cost. n. 106/1962, cit.

15 Da ultimo, C. cost. n. 178/2015, cit.; sia consentito il rinvio a G. CEnTAmo$\mathrm{RE}$, Legge e autonomia collettiva: una critica della dottrina dell'inderogabilità bilaterale, in Lav. Dir., 2015, p. 491 ss. 
sono stati nella sostanza considerati appropriati dalla Corte costituzionale $^{16}$.

Tuttavia, se queste considerazioni potrebbero adattarsi a una riflessione per così dire più generale sulla contrattazione collettiva, esse non sembrano risolutive con riferimento alla definizione delle unità contrattuali, che se da una parte è esercizio di contrattazione collettiva, dall'altra si proietta altresì sul profilo dell'organizzazione. L'interrogativo in sé è semplice - darvi una risposta lo è molto meno -: un intervento eteronomo con il quale si stabiliscano (non le categorie contrattuali, bensi) i meccanismi mediante i quali definire le categorie contrattuali, andrebbe considerato alla stregua di un intervento sull'organizzazione sindacale (sottoposto a limiti rigorosissimi) o sulla contrattazione collettiva (con limiti non di scarso momento, ma meno stringenti)? Vero è che da questo cul-de-sac si potrebbe uscire affermando come sia precluso interferire in qualsiasi modo con la definizione delle unità negoziali da parte dei soggetti che stipulano i contratti collettivi: poiché oggetto di protezione diretta nell'art. 39, c. 1, Cost., al pari della stessa organizzazione, tale posizione giuridica sarebbe semplicemente incomprimibile, sia pure in modo lieve, da parte dei pubblici poteri, pena la violazione del principio di libertà sindacale.

Questo, in estrema sintesi, sembra essere l'orientamento prevalente da decenni nella dottrina italiana: pur essendo condivisibile, nei suoi presupposti e, in qualche misura, negli svolgimenti, all'esito di questa ricerca è possibile svolgere alcune considerazioni allo scopo di ridimensionarne e relativizzarne la portata.

Le riflessioni svolte nel capitolo III, che si sono avvalse di alcuni spunti di diritto sindacale comparato e di validi esempi tratti dal diritto interno (in particolare, dal dibattito sull'attuazione dell'art. 39 Cost., dal d.lgs. n. 165/2001 e dalla 1. n. 146/1990), hanno consentito di interrogarsi più a fondo sulla portata del principio costituzionale di libertà, in relazione alle attività di definizione dei perimetri negoziali. Vi si può trarre la conclusione che la norma costituzionale non esclude l'ammissibilità di una soluzione "non formalistica" del problema della categoria ${ }^{17}$.

16 Rispettivamente, con le pronunce n. 344/1996, cit., n. 309/1997, cit.

17 Più in generale, l'eventualità di prendere in considerazione una lettura "non formalistica" dell'art. 39 Cost., è suggerita ormai da ampi settori della dottrina e, in qualche misura, dalla stessa Corte costituzionale. Pur non avendo mai apertamente abbandona- 
Se non ci si ferma ad una lettura dell'art. 39 Cost. che ne enfatizzi la sola dimensione di libertà, a detrimento degli equilibri complessivi della norma, è possibile riconoscere che la definizione delle unità negoziali può essere validamente realizzata in forme compatibili con il principio di cui al comma primo e non fedelmente sovrapponibili a quelle preconizzate dal comma quarto. Quel che occorre è «ricercare la soluzione che possa contemperare meglio una delimitazione univoca dei perimetri contrattuali con il principio di libertà sindacale» ${ }^{18}$, anche «alla luce d'un'esperienza durata cinquant'anni e dunque delle consolidate tendenze a cui è ascrivibile l'obsolescenza dei [...] frammenti normativi [dell'art. 39] che più impietosamente ne denunciano l'età avanzata» ${ }^{19}$.

Il punto fermo è che il principio di libertà sindacale non ammetterebbe in alcun caso un'estromissione delle parti sociali dai processi di definizione delle unità contrattuali. È questa, per così dire, una delle prerogative il cui esercizio si colloca più in prossimità del "nucleo duro" dell'art. 39 Cost. La sostanza della questione sta allora nel valorizzare le prassi di definizione delle unità negoziali da parte delle organizzazioni contrapposte, mediante dispositivi che attribuiscano loro questa prerogativa, ma al tempo stesso garantiscano che non vi siano eccessive incoerenze nella struttura negoziale ${ }^{20}$. A ben guardare,

to il proprio consolidato orientamento, per il quale l'attribuzione di effetti erga omnes ai contratti collettivi non può essere disposta con procedure diverse da quelle previste dall'art. 39, la Corte costituzionale ha nel tempo affinato tale posizione. Sono state, negli anni, respinte una serie di censure di legittimità costituzionale, che avevano investito discipline di legge che, in una varietà di soluzioni, consentono di estendere ultra partes gli effetti dei contratti collettivi. Si allude in particolare a: C. Cost. n. 270/1987, cit. (fiscalizzazione degli oneri sociali); C. Cost. 30 giugno 1994, n. 268, in Riv. It. Dir. Lav., 1995, II, p. 237, nota E. Manganiello, Riv. Giur. Lav., 1994, II, p. 661, nota C. De MarCHIS (criteri di scelta nei licenziamenti collettivi); C. Cost. n. 344/1996, cit. (sciopero nei servizi pubblici essenziali); C. cost. n. 309/1997, cit. (contrattazione collettiva per il lavoro pubblico); C. cost. n. 51/2015, cit. (trattamento economico dei soci lavoratori di cooperativa). Per qualche spunto, cfr. L. Zoppoli, Una nuova Costituzione per il sistema sindacale italiano? Riflessioni introduttive, in L. Zoppol, A. Zoppoli, M. Delfino (a cura di), Una nuova Costituzione per il sistema di relazioni sindacali?, cit., p. 11 ss.

18 T. Treu, La questione salariale: legislazione sui minimi e contrattazione collettiva, cit., p. 22.

19 Così, U. Romagnoli, L'inutile necessità di una disputa, in Dir. Lav. Rel. Ind., 1996, p. 13.

20 Sembra di cogliere un cenno, in tal senso, da parte di M. Magnani, Riflessioni sulla misurazione della rappresentanza datoriale nell'ordinamento statale e intersindacale, cit., pp. 5-10. 
la questione della categoria è aperta a una varietà di soluzioni tecniche e di politica del diritto.

Tuttavia, l'eventualità di un'attuazione globale della norma costituzionale, oltre a non sembrare all'orizzonte nel panorama politicosindacale, non sarebbe, dopo settant'anni di sviluppo autonomo del sistema di relazioni industriali, facilmente conciliabile con quest'ultimo. L'elevato livello di pluralismo organizzativo, sia tra le associazioni dei lavoratori sia tra quelle che rappresentano le imprese, si riflette in una struttura contrattuale densa, articolata e caratterizzata da intersezioni e sovrapposizioni tra le aree negoziali che non potrebbero essere risolte d'imperio dalla legge: è difficile, invero, anche soltanto progettare un intervento di attuazione dell'art. 39 Cost., prima che le parti sociali siano riuscite a riportare ordine nella struttura contrattuale. Parrebbe più realizzabile una soluzione intermedia, che valorizzi ed estenda l'esperienza dei rinvii all'autonomia collettiva, cercando al contempo di correggerne alcune difficoltà di funzionamento che la prassi di questi ultimi anni ha messo in luce.

A uno sguardo d'insieme si coglie la profondità dell'intreccio tra legge e autonomia collettiva, a un punto tale da mettere in dubbio la stessa collocazione sistematica del contratto collettivo nell'ordine delle fonti della materia ${ }^{21}$.

Vero è che se si intende la locuzione fonti in modo più generico $\mathrm{o}$, se si vuole, a-tecnico, come congegni dai quali ricavare la disciplina dei rapporti di lavoro, l'inclusione dei contratti collettivi in questo sistema è più che nelle cose: è nel codice genetico della materia ${ }^{22}$. La legge non potrebbe regolare questo settore della vita sociale con la sistematicità ed il tempismo che le parti sociali possono garantire mediante la continuativa determinazione per via contrattuale di una "regola comune" di lavoro $^{23}$. È presumibile, inoltre, che i contratti collettivi esprimano livelli di trattamento economico e normativo adeguati nella loro misura e accettabili tra gli attori economici, consideratane la provenienza dagli equilibri trovati nel mercato dalle organizzazioni di interessi contrappo-

21 C. Zoli, Contratto collettivo come fonte e contrattazione collettiva come sistema di produzione di regole, cit.

22 S. e B. Weвв, La democrazia industriale, Torino, Utet, 1912.

23 M. Pedrazzoli, Qualificazioni dell'autonomia collettiva e procedimento applicativo del giudice, cit. 
ste, per unità negoziali liberamente definite ${ }^{24}$. Tutto ciò altro non significa se non «piegarsi al pragmatismo della regola non scritta per cui la legge non può fare a meno del contratto collettivo» ${ }^{25}$. Vale a dire, cioè, che pur potendo il legislatore porre direttamente una disciplina, per situazioni considerate meritevoli di intervento, è valutato conveniente rimettersi «a uno strumento normativo più flessibile, qual è il contratto collettivo» ${ }^{26}$, sia pure al costo di un «allungamento delle vie della legittimazione ${ }^{27}$ nei processi di formazione del diritto.

Ciò si è risolto in una politica di favore per l'estensione degli effetti dei contratti collettivi nel sistema economico, perpetrata dal legislatore, dalla giurisprudenza e dall'autorità amministrativa lungo tutto l'arco dell'esperienza repubblicana ${ }^{28}$, rimediando alle deficienze organizzative cui il sindacalismo, sia dei lavoratori sia dei datori di lavoro, si espone, in alcune zone marginali ${ }^{29} \mathrm{o}$, se si preferisce, aree calde $^{30}$ del mercato. Laddove le parti sociali non abbiano esse stesse la forza di assicurare ampi margini di applicazione dei contratti collettivi, se ne favorisce comunque la diffusione ${ }^{31}$, in modo compatibile con la prevalente ricostruzione privatistica ${ }^{32}$.
24 M. GRANDI, In difesa della rappresentanza sindacale, cit., p. 640.
25 Così, U. Romagnoli, Il contratto collettivo, cit., p. 236.
26 G. Vardaro, Differenze di funzioni e di livelli fra contratti collettivi, in Lav. Dir., 1987, p. 259.

27 L. Mengoni, La questione del «diritto giusto» nella società post-liberale, cit., p. 24. V., altresì, M. NAPOLI, Autonomia collettiva e autonomia individuale nelle più recenti riforme, in Dir. Lav. Rel. Ind., 2004, p. 603, che ne svolge una lettura in termini di sussidiarietà orizzontale, in favore dei soggetti più prossimi ai contesti nei quali le discipline devono essere osservate.

28 Cfr. E. GHERA, Le c.d. clausole sociali: evoluzione di un modello di politica legislativa, cit., pp. 134-136.

29 M. Rusciano, Il contratto collettivo, cit., p. 76. p. 259.

30 G. Vardaro, Differenze di funzioni e di livelli fra contratti collettivi, cit.,

31 P. Pascucci, Giusta retribuzione e contratti di lavoro, oggi, cit., p. 79. Non è un caso che in Italia il tasso di copertura contrattuale sia tra i più alti dell'area Ocse (circa l'80\%), al punto da consentire all'organizzazione sovranazionale di includere il nostro tra gli ordinamenti giuridici dotati di erga omnes di fatto (OECD, Employment Outlook 2017, Paris, Oecd publishing, 2017, p. 141).

32 Cfr. P. Tosi, Contratto collettivo e rappresentanza sindacale, in Pol. Dir., 1985, pp. 364, 366; M. Roccella, Contrattazione collettiva, azione sindacale, problemi di regolazione del mercato del lavoro, cit., pp. 353-354; C. Zol, Il corrispettivo della prestazione lavorativa, in L. Nogler, M. TRemolada, C. Zoli (a cura di), La riforma della posizione giuridica del socio lavoratore di cooperativa, cit., p. 409; M. GRANDI, In di- 
Questa esperienza ormai pluridecennale di rinvii all'autonomia collettiva per la determinazione di un parametro del trattamento, quantomeno economico, potrebbe essere valorizzata con un rinvio più ad ampio raggio, rispetto a quelli "mirati" effettuati sinora dal legislatore ${ }^{33}$. In particolare, si tratterebbe di attribuire apertamente alle parti sociali il compito di stabilire i trattamenti economici complessivi adeguati ai diversi segmenti del mercato del lavoro, unitamente alla prerogativa fondamentale di definire le aree contrattuali, nel rispetto dell'art. 39, c. 1 , Cost.; a tal fine, potrebbe altresì prendersi in considerazione la possibilità di un intervento di "semplificazione" di un'autorità terza, che si limiti ad esplicitare i trattamenti previsti dalla contrattazione collettiva, come nell'esperienza delle tabelle che il Ministero del lavoro predispone nel settore dei contratti pubblici: ciò non toglie, peraltro, che gioverebbe, per un verso, una previa convergenza, sia pure di massima, tra le principali associazioni delle imprese, circa le aree di rispettiva influenza negoziale e, per l'altro, che entrassero finalmente a regime i meccanismi di misurazione e verifica della rappresentatività delle organizzazioni che stipulano i contratti collettivi.

fesa della rappresentanza sindacale, cit., p. 645; A. LASSANDARI, Il contratto collettivo aziendale e decentrato, cit., pp. 250-251; U. CARABELLI, V. LECCESE, Una riflessione sul sofferto rapporto tra legge e autonomia collettiva, in AA.VV., Studi in onore di Giorgio Ghezzi, I, Padova, Cedam, 2006, p. 345 ss., p. 382; F. CARINCI, Diritto privato e diritto del lavoro: uno sguardo dal ponte, in WP CSDLE “Massimo D’Antona”.IT, 54/2007, p. 55; P. Bellocchi, Il contratto collettivo di diritto comune, cit., p. 281; M.G. GarofaLo, Per una teoria giuridica del contratto collettivo, cit., pp. 524-527; F. SANTONI, Contrattazione collettiva e principio di maggioranza, in Riv. It. Dir. Lav., 2013, I, pp. 93, 96; M. BARBIERI, In tema di legittimità costituzionale del rinvio al CCNL delle organizzazioni più rappresentative nel settore cooperativo per la determinazione della retribuzione proporzionata e sufficiente, cit. Con particolare riferimento alle disposizioni che rinviano all'autonomia collettiva ai fini della determinazione di un parametro del trattamento, la giurisprudenza di legittimità è costante nell'affermarne la compatibilità con la ricostruzione privatistica del contratto collettivo. Tra le molte, v., in materia di minimale contributivo, Cass. SSUU n. 11199/2002, cit.; in materia di fiscalizzazione degli oneri sociali e sgravi contributivi, Cass. 11 gennaio 2000, n. 227, in Notiz. Giur. Lav., 2000, p. 393; in materia di appalti pubblici, Cass. n. 5189/2019, cit.; in materia di soci lavoratori di cooperative, v. Cass. n. 28289/2019, cit., Cass n. 4951/2019, cit.

33 Cfr. in tal senso P. PAscucci, Giusta retribuzione e contratti di lavoro, oggi, cit., pp. 76-77. 


\section{BiBLIOGRAFIA}

AA.VV., La categoria e la contrattazione collettiva, Milano, Giuffrè, 1964. AA.VV., Categorie professionali e contratti collettivi, Suppl. Notiz. Giur. Lav., Roma, Assicredito, 1990.

AA.VV., L'attuazione degli articoli 39 e 46 della Costituzione. Tre proposte a confronto, Quaderni Riv. Giur. Lav., Roma, Ediesse, 2016.

AA.VV., Diritto di sciopero e rappresentatività sindacale, Milano, Wolters Kluwer-Cedam, 2018.

Accornero A., La parabola del sindacato, Bologna, Il Mulino, 1992.

Alaimo A., La contrattazione collettiva nel settore pubblico tra vincoli, controlli e "blocchi": dalla "riforma Brunetta" alla "manovra finanziaria” 2010, in Lav. Pubb. Amm., 2010, p. 287 ss.,

Albini P., A proposito di rappresentanza datoriale, in Dir. Lav. Rel. Ind., 2017, p. 265 ss.

Allamprese A., Bonardi O. (a cura di), Logistica e lavoro, Quaderni Riv. Giur. Lav., Roma, Ediesse, 2018.

Alleva P.G., I profili giuslavoristici della nuova disciplina del socio lavoratore di cooperativa, in Riv. Giur. Lav., 2001, I, p. 353 ss.

ALvino I., Il lavoro nelle reti d’imprese: profili giuridici, Milano, Giuffrè, 2014.

Alvino I., I rinvii legislativi al contratto collettivo, Napoli, Jovene, 2018.

Alvino I., Imberti L., Contratto collettivo leader e rappresentanza datoriale, in Lab. Law Iss., 2018, 2, p. 3 ss.

Aguarone A., Aspirazioni tecnocratiche del primo fascismo, in Nord e Sud, 1964, 9, p. 117 ss.

Assanti C., Rilevanza e tipicità del contratto collettivo nella vigente legislazione italiana, Milano, Giuffrè, 1967. 
Avanzi F., Dimensione e qualità dei contratti. Spunti e riflessioni dall'analisi Cnel-Inps, in Boll. Adapt, 29 luglio 2019, n. 29.

Bagolini L., Mistero ed esperienza sociale e giuridica, in Riv. Trim. Dir. Proc. Civ., 1961, p. 946 ss.

Balandi G.G., Le «clausole a favore dei lavoratori» e l'estensione dell'applicazione del contratto collettivo, in Riv. Trim. Dir. Proc. Civ., 1973, p. 698 ss.

Balducci C., Il sindacato, I, L'organizzazione sindacale, Torino, Utet, 1984.

Ballestrero M.V., Art. 2, in Romagnoli U., Ballestrero M.V., Art. 40 Supplemento: Legge 12 giugno 1990 n. 146. Norme sull'esercizio del diritto di sciopero nei servizi pubblici essenziali, in BRANCA GIU. (a cura di), Commentario della Costituzione, Bologna-Roma, Zanichelli-Società editrice del Foro italiano, 1994. p. 85 ss.

Ballestrero M.V., Diritto sindacale, Torino, Giappichelli, 2018.

Ballestrero M.V., Interesse collettivo e conflitto, in Lav. Dir., 2018, p. 411 ss.

Balletti B., Contributo alla teoria della autonomia sindacale, Milano, Giuffrè, 1963.

BARBIERI M., Intervento, in AA.VV., Autonomia collettiva e occupazione. Atti del XII Congresso nazionale di diritto del lavoro Milano, 23-25 maggio 1997, Milano, Giuffrè, 1997, p. 176 ss.

Barbieri M., Problemi costituzionali della contrattazione collettiva nel lavoro pubblico, Bari, Cacucci, 1997.

Barbieri M., Cinque anni dopo: il rapporto di lavoro del socio di cooperativa tra modifiche legislative, dottrina e giurisprudenza, in CuRzı P. (a cura di), Lavoro e diritti a tre anni dalla legge 30/2003, Bari, Cacucci, 2005, p. 523 ss.

BARbieri M., Note critiche sul testo unico sulla rappresentanza sindacale, in Zoppoli L., Zoppoli A., Delfino M. (a cura di), Una nuova Costituzione per il sistema di relazioni sindacali?, Napoli, Editoriale Scientifica, 2014, p. 211 ss.

BARBIERI M., In tema di legittimità costituzionale del rinvio al Ccnl delle organizzazioni più rappresentative nel settore cooperativo per la determinazione della retribuzione proporzionata e sufficiente, in Riv. Giur. Lav., 2015, II, p. 493 ss.

BARBIERI M., La contrattazione collettiva nazionale oggi: caratteri, metamorfosi, criticità, in Lassandari A., Martelloni F., Tullini P., Zoli C. (a cura di), La contrattazione collettiva nello spazio economico globale, Bologna, Bononia University Press, 2017, p. 3 ss. 
Barbieri M., Della subordinazione dei ciclofattorini, in Lab. Law. Iss., 2019, 2, p. I.1 ss.

Barbieri M., Garofalo M.G., La contrattazione collettiva, in Carinci F. (dir. da), Il lavoro alle dipendenze delle amministrazioni pubbliche dal D.LGS. 29/1993 alla Finanziaria 1995. Commentario, I, Milano, Giuffrè, 1995, p. 689 ss.

Battistell S. et al., Structural characteristics and industrial relations in the pork value chain: the case of Italy, in Campanella P., Dazzi D. (eds), Meat-up Ffire Fairness freedom and industrial relations across Europe: up and down the meat value chain, Milano, Franco Angeli, 2020, p. 139 ss.

Bavaro V., Azienda contratto sindacato, Bari, Cacucci, 2012.

Bavaro V., Rappresentanza e rappresentatività sindacale, in Dir. Lav. Merc., 2012, p. 31 ss.

Bavaro V., Il principio maggioritario nelle relazioni industriali, in Lav. Dir., 2014, p. 3 ss.

BAVARO V., Il contratto nazionale dei metalmeccanici 2016: una prospettiva sulle relazioni industriali italiane, in Dir. Lav. Rel. Ind., 2017, p. 729 ss.

Bavaro V., Laforgia S., Contrattazione collettiva e "prossimità delle imprese". La struttura del contratto collettivo di filiera, distretto, rete d'imprese, in CARINCI M.T. (a cura di), Dall'impresa a rete alle reti d'impresa. Scelte organizzative e diritto del lavoro, Milano, Giuffrè, 2015, p. 107 ss.

Baylos Grau A., Zoppoli L. (a cura di), La libertà sindacale nel mondo: nuovi profili e vecchi problemi. In memoria di Giulio Regeni, Quaderni Dir. Lav. Merc., Napoli, Editoriale Scientifica, 2019.

Bechter B., Brandl B., Meardi G., Sectors or countries? Typologies and levels of analysis in comparative industrial relations, in Eur. J. Ind. Rel., 2012, 3, p. 185 ss.

Bellardi L., L'attuazione dell'accordo quadro: pluralità dei sistemi contrattuali ed eterogenesi dei fini. Alcune note di sintesi, in Dir. Lav. Rel. Ind., 2010, p. 387 ss.

BELLARDI L., Il sistema di rappresentanza imprenditoriale e la struttura della contrattazione collettiva, in CARRIERI M., TREU T. (a cura di), Verso nuove relazioni industriali, Bologna, Il Mulino, 2013, p. 241 ss.

Bellardi L., Sistema politico, legge e relazioni industriali: dalla promozione all'esclusione?, in AA.VV., Studi in memoria di Mario Giovanni Garofalo, 1, Bari, Cacucci, 2015, p. 103 ss.

Bellardi L., Relazioni industriali e contrattazione collettiva: criticità e prospettive, in Lav. Dir., 2016, p. 939 ss. 
Bellavista A., La clausola sociale dell'art. 36 st. lav. e l'art. 2070 c.c., in Riv. It. Dir. Lav., 1999, II, p. 462 ss.

Bellavista A., Contrattazione collettiva e partecipazione sindacale nella riforma Madia, in Giur. It., 2018, p. 1005 ss.

Bellocchi P., Il contratto collettivo di diritto comune, in Proia G. (coord. da), Il diritto sindacale, in CARINCI F. (a cura di), Il lavoro subordinato, in Bessone M. (dir. da), Trattato di diritto privato, Torino, Giappichelli, 2007, p. 261 ss.

Bellocchi P., La libertà sindacale, in Proia G. (a cura di), Organizzazione sindacale e contrattazione collettiva, in CARINCI F., PERSIANI M. (dir. da), Trattato di diritto del lavoro, Padova, Cedam, 2014, p. 3 ss.

Bellocchi P., Art. 2070, in Amoroso G., Di Cerbo V., Maresca A. (a cura di), Il diritto del lavoro, 1, La Costituzione, il Codice civile e le leggi speciali, V ed., Milano, Giuffrè, 2017, p. 661 ss.

Bellomo S., Art. 36, in Amoroso G., Di Cerbo V., Maresca A. (a cura di), Il diritto del lavoro, 1, La Costituzione, il Codice civile e le leggi speciali, V ed., Milano, Giuffrè, 2017, p. 164 ss.

BetтI E., Teoria generale del negozio giuridico, Torino, Utet, 1960, II ed., III rist.

Bigiavi W., «Normalità» e «anormalità» nella costruzione giuridica, in Riv. Dir. Civ., 1968, I, p. 518 ss.

BLANPain R., Labour law in Belgium, IV ed., Alphen aan den Rijn, Kluwer law international, 2012.

Bollani A., Contratto collettivo e interpretazione, Padova, Cedam, 2004.

Bologna Se., CuRI S., Relazioni industriali e servizi di logistica: uno studio preliminare, in Dir. Lav. Rel. Ind., 2019, 125 ss.

Bologna Si., La contrattazione collettiva negli Stati Uniti dal Wagner act ai nostri giorni, in Riv. It. Dir. Lav., 2016, I, p. 421 ss.

BONARDI O., Tra governance della global value chain e frammentazione del tessuto produttivo nazionale: quale regolamentazione per il lavoro nel settore della logistica?, in Allamprese A., Bonardi O. (a cura di), Logistica e lavoro, Quaderni Riv. Giur. Lav., Roma, Ediesse, 2018, p. $20 \mathrm{ss}$.

Bordogna L., L'accordo Confindustria-sindacati del 9 marzo 2018 su relazioni industriali e contrattazione collettiva, in Dir. Lav. Rel. Ind., 2019, p. 37 ss.

Borgogelli F., Rappresentatività sindacale e consenso nella regolazione dello sciopero nei servizi essenziali, in Riv. Giur. Lav., 2007, I, p. 675 ss. 
Borgogelli F., Appalti pubblici e dumping salariale: un caso di subordinazione dell'autonomia collettiva, in Lav. Dir., 2016, p. 985 ss.

Bortone R., Curzio P., Il contratto collettivo, Torino, Utet, 1984.

Branca Gio., L'associazione sindacale, Milano, Giuffrè, 1960.

Branca Gio., La prestazione di lavoro in società collegate, Milano, Giuffrè, 1965.

Buonocore V., Impresa (diritto privato), in Enc. Dir., Annali, I, 2007, p. 760 ss.

CAFFIO D., Appalto, costo del lavoro e contratto collettivo di riferimento, in Garofalo D. (a cura di), Appalti e lavoro, I, Disciplina pubblicistica, Torino, Giappichelli, 2017, p. 849 ss.

Calogero G., La polemica sui concetti giuridici, in Riv. Dir. Comm., 1945, I, p. 112 ss.

Cammarata A. E., Il significato e la funzione del «fatto» nell'esperienza giuridica (1929), in Cammarata A. E., Formalismo e sapere giuridico. Studi, Milano, Giuffrè, 1963, p. 247 ss.

Campanella P., Rappresentatività sindacale: fattispecie ed effetti, Milano, Giuffrè, 2000.

Campanella P., CARINCi M.T., L'attuazione della legge delega «Bassanini»: il d.lgs. $n$. 396/1997 in tema di contrattazione collettiva e rappresentanza sindacale nel pubblico impiego, in Lav. Pubb. Amm., 1998, p. 65 ss. CAPponi F., lavoro tramite piattaforma digitale: prima lettura del d.l. $n$. 101/2019 convertito in l. n. 128/2019, in Dir. Rel. Ind., 2019, p. 1231 SS.

CARabelli U., La "riforma Brunetta": un breve quadro sistematico delle novità legislative e alcune considerazioni critiche, in WP CSDLE “Massimo D’Antona”.IT, 101/2010.

CARABELLI U., Legge sindacale e ordinamento intersindacale: alcune riflessioni sparse, in AA.VV., L'attuazione degli articoli 39 e 46 della Costituzione. Tre proposte a confronto, Quaderni Riv. Giur. Lav., Roma, Ediesse, 2016, p. 183 ss.

CARABelli U., LeCCESE V., Una riflessione sul sofferto rapporto tra legge e autonomia collettiva, in AA. VV., Studi in onore di Giorgio Ghezzi, I, Padova, Cedam, 2005, p. 345 ss.

CARINCI F., Il conflitto collettivo nella giurisprudenza costituzionale, Milano, Giuffrè, 1971.

CARINCI F., Diritto privato e diritto del lavoro: uno sguardo dal ponte, in WP CSDLE “Massimo D'Antona”.IT, 54/2007.

CARINCI F., Una dichiarazione d'intenti: l'Accordo Quadro 22 gennaio 
2009 sulla riforma degli assetti contrattuali, in Riv. It. Dir. Lav., 2009, I, p. 177 ss.

CARINCI F., Alice non abita più qui (a proposito e a sproposito del "nostro" diritto sindacale), in Dir. Lav. Rel. Ind., 2013, p. 674 ss.

CARINCI F., Contrattazione e contratto collettivo nell'impiego pubblico "privatizzato", in Lav. Pubb. Amm., 2013, p. 493 ss.

CARINCI F. (a cura di), Legge o contrattazione? Una risposta sulla rappresentanza sindacale a Corte costituzionale n. 231/2013, Adapt Labour Studies, E-Book Series, n. 20, 2014.

CARrieri M., Feltrin P., Al bivio. Lavoro, sindacato e rappresentanza nell'Italia d'oggi, Roma, Donzelli, 2016.

Carta C., La crisi della contrattazione di settore in Germania, in Lav. Dir., 2019, p. 645 ss.

CARTA C., La funzione di sostegno alla competitività degli accordi aziendali peggiorativi. Il contributo dell'analisi comparata con $i$ modelli francese e tedesco, in Variaz. Temi Dir. Lav., 2019, p. 603 ss.

Carullo V., Diritto sindacale transitorio, Milano, Giuffrè, 1960.

Caruso B., Rappresentanza sindacale e consenso, Milano, Franco Angeli, 1992.

CARuso B., Per un intervento eteronomo sulla rappresentanza sindacale: se non ora quando!, in WP CSDLE “Massimo D'Antona”.IT, 206/2014.

Caruso B., Testo Unico sulla Rappresentanza, in WP CSDLE "Massimo D'Antona”.IT, 226/2014.

Caruso B., Lo Faro A., Contratto collettivo di lavoro (Voce per un dizionario), in WP CSDLE “Massimo D'Antona”.IT, 97/2010.

Cassì V., La rappresentanza unitaria dei sindacati registrati, in Riv. Dir. Lav., 1950, I, p. 159 ss.

CAStelvetri L., Gli strumenti e i contenuti delle relazioni industriali nel sistema intersindacale: la contrattazione collettiva, in PESCHIERA F. (a cura di), Sindacato, industria e Stato nel dopoguerra: storia delle relazioni industriali in Italia dal 1943 al 1948, Firenze, Le Monnier, 1976, p. 384 ss.

Cataudella A., Fattispecie, in Enc. Dir., XVI, 1967, p. 926 ss.

Cella G.P., Quali cambiamenti per le relazioni industriali italiane?, in Lav. Dir., 2007, p. 227 ss.

Cella G.P., Treu T., Relazioni industriali e contrattazione collettiva, Bologna, Il Mulino, 2009.

Centamore G., Legge e autonomia collettiva: una critica della dottrina dell'inderogabilità bilaterale, in Lav. Dir., 2015, p. 491 ss.

CessaRI A., L'interpretazione dei contratti collettivi, Milano, Giuffrè, 1963. 
CHIEco P., La natura organizzatoria delle norme di contrattualizzazione dei rapporti di lavoro alle dipendenze della p.a.: brevi spunti sistematici, in AA.VV., Le trasformazioni dei rapporti di lavoro pubblico e il sistema delle fonti: atti delle Giornate di studio di diritto del lavoro: L’Aquila, 31 maggio-1 giugno 1996, Milano, Giuffrè, 1997, p. 299 ss. Ciucciovino S., Mettere ordine nella giungla dei ccnl: un'esigenza indifferibile, in Dir. Lav. Rel. Ind., 2018, p. 227 ss.

CNel, $3^{\circ}$ Report periodico dei Contratti Collettivi Nazionali di Lavoro depositati nell'archivio CNEL, aggiornamento marzo 2016.

CNEL, Notiziario dell'Archivio contratti, n. 30, nuova serie, luglio 2018.

CNel, $9^{\circ}$ Report periodico dei Contratti Collettivi Nazionali di Lavoro depositati nell'archivio CNEL, aggiornamento giugno 2019.

CNel, $10^{\circ}$ Report periodico dei Contratti Collettivi Nazionali di Lavoro depositati nell'archivio CNEL, aggiornamento dicembre 2019.

CNeL, Notiziario dell'Archivio contratti, n. 33, nuova serie, novembre 2019. Commissione Europea, Country report 2019 - Italy.

Corazza L., Contractual integration e rapporti di lavoro, Padova, Cedam, 2004.

Corazza L., La nuova nozione di appalto nel sistema delle tecniche di tutela del lavoratore, in WP CSDLE “Massimo D'Antona”. IT, 93/2009.

Cordova E., Collective bargaining, in Blanpain R. (ed.), Comparative labour law and industrial relations, Dordrecht, Springer, 1982, p. 220 ss.

Curzio P., Autonomia collettiva e sciopero nei servizi essenziali, Bari, Cacucci, 1992.

Curzıo P., Nomofilachia e autonomia collettiva, in Dir. Lav. Rel. Ind., 2014, p. 753 ss.

D’Addio F., Tiraboschi M., Part-time senza vincolo di orario minimo e sindacato comparativamente più rappresentativo, in Dir. Rel Ind., 2017, p. 882 ss.

D'Antona M., Diritto sindacale in trasformazione, in D'Antona M. (a cura di), Letture di diritto sindacale, Napoli, Jovene, 1990, p. I ss.

D'Antona M., Crisi e prospettive della regolamentazione extralegislativa del diritto di sciopero nei servizi pubblici essenziali, in Riv. Giur. Lav., 1991, I, p. 417 ss.

D’Antona M., Lavoro pubblico e diritto del lavoro: la seconda privatizzazione del pubblico impiego nelle «leggi Bassanini», in Lav. Pubb. Amm., 1998, p. 35 ss.

D’Antona M., Il quarto comma dell'art. 39 della Costituzione, oggi, in Dir. Lav. Rel. Ind., 1998, p. 665 ss. 
D'Ascola S., Controllo del contratto collettivo in Cassazione: spigolature del nuovo orientamento, in Arg. Dir. Lav., 2016, p. 912 ss.

D’ONGHIA M., I soggetti della contrattazione collettiva di parte pubblica: Comitati di settore e ARAN, in CARINCI F., Mainardi S. (a cura di), La terza riforma del Lavoro Pubblico: commentario al D.Lgs. 27 ottobre 2009, n. 150, aggiornato al "Collegato lavoro", Milanofiori-Assago, Ipsoa, 2011, p. 367 ss.

D’Onghia M., Ricci M. (a cura di), Lo sciopero nei servizi pubblici essenziali, Milano, Giuffrè, 2003.

Davies P., Freedland M., Kahn-Freund's Labour and the law, London, Stevens, 1983.

De Luca Tamajo R., L'evoluzione dei contenuti e delle tipologie della contrattazione collettiva, in Riv. It. Dir. Lav., 1985, I, p. 16 ss.

De Luca Tamajo R., Incertezze e contraddizioni del Diritto sindacale italiano: è tempo di regolamentazione legislativa, in Riv. It. Dir. Lav., 2018, I, p. 273 ss.

De Mozzi B., Terzo settore e contrattazione collettiva, in Variaz. Temi Dir. Lav., 2019, p. 1015 ss.

Del Punta R., Il contratto aziendale nella dottrina italiana, in Riv. It. Dir. Lav., 1989, I, p. 248 ss.

Del Punta R., La sospensione del rapporto di lavoro. Malattia, infortunio, maternità, servizio militare. Artt. 2110-2111, Milano, Giuffrè, 1992.

Del Punta R., Note sparse sul Testo Unico sulla rappresentanza, in Dir. Rel. Ind., 2014, p. 673 ss.

Del Punta R., Tre domande e una chiosa sulla legge sindacale, in F. CARINCI (a cura di), Legge o contrattazione? Una risposta sulla rappresentanza sindacale a Corte costituzionale n. 231/2013, Adapt Labour Studies, E-Book Series, n. 20, 2014, p. 159 ss.

Dell'Olio M., L'organizzazione e l'azione sindacale, Padova, Cedam, 1980.

Dell'Olio M., Ancora sulla rappresentatività, e su maggioranza e proporzionalità, organizzazione sindacale, categoria, nello statuto dei lavoratori e nel sistema, in Giur. Cost., 1988, II, p. 1433 ss.

Dell’Olio M., Legge e contratto collettivo: autorità, funzione, libertà, in AA.VV., Le trasformazioni dei rapporti di lavoro pubblico e il sistema delle fonti: atti delle Giornate di studio di diritto del lavoro: L'Aquila, 31 maggio-1 giugno 1996, Milano, Giuffrè, 1997, p. 251 ss.

Della Rocca G., Il sindacato, in Cella G.P., Treu T., Le nuove relazioni 
industriali. L'esperienza italiana nella prospettiva europea, Bologna, Il Mulino, 1998, p. 95 ss.

Dorigatti L., Mori A., L'impatto delle scelte datoriali sulle condizioni di lavoro e sulle diseguaglianze: disintegrazione verticale, esternalizzazioni e appalti, in Soc. Lav., 2016, p. 190 ss.

Dorssemont F., On the representative status of Belgian employers' organizations for the purpose of collective bargaining, in Dir. Lav. Rel. Ind., 2017, p. 253 ss.

Esposito C., Lo Stato e i sindacati nella Costituzione italiana, in Esposito C., La Costituzione italiana. Saggi, Padova, Cedam, 1954, p. 151 ss.

Esposito M., Il contratto collettivo del lavoro pubblico: così speciale, così reale, in Zoppoli L., Zoppoli A., Delfino M. (a cura di), Una nuova Costituzione per il sistema di relazioni sindacali?, Napoli, Editoriale Scientifica, 2014, p. 87 ss.

Ewing K. D., Hendy J., Jones C. (eds.), Rolling out the Manifesto for Labour Law, Liverpool, the Institute of Employment Rights, 2018.

Faioli M., Sindacato statunitense, in Dig. Comm., Agg., 2015, p. 498 ss.

Ferraro G., Ordinamento, ruolo del sindacato, dinamica contrattuale di tutela, Padova, Cedam, 1981.

Ferraro G., La classificazione delle imprese ai sensi dell'art. 49 della legge n. 88/1989, in Riv. It. Dir. Lav., 1990, I, p. 157 ss.

Ferraro G., Sul rinnovato "sistema" di relazioni industriali, in Zoppol L., Zoppoli A., Delfino M. (a cura di), Una nuova Costituzione per il sistema di relazioni sindacali?, Napoli, Editoriale Scientifica, 2014, p. 295 ss.

Ferraro G., Teorie e cronache del diritto sindacale e autorità del punto di vista giuridico, in Arg. Dir. Lav., 2016, p. 16 ss.

Fiorillo L., Le fonti di disciplina del rapporto di lavoro pubblico, Padova, Cedam, 1990.

Flammia R., Contributo all'analisi dei sindacati di fatto. I. Autotutela degli interessi di lavoro, Milano, Giuffrè, 1963.

Flammia R., Problemi circa l'ambito di applicazione dei contratti collettivi, in AA.VV., Categorie professionali e contratti collettivi, Suppl. Notiz. Giur. Lav., Roma, Assicredito, 1990, p. 21 ss.

FoA V., Cento anni di sindacato in Italia (1973), in FoA V., Per una storia del movimento operaio, Torino, Einaudi, 1980, p. 92 ss.

ForLIVESI M., La rappresentatività datoriale: funzioni, modelli, indici di accertamento, in Lav. Dir., 2018, p. 521 ss.

Franciosi U., Il caso Castelfrigo, in Allamprese A., Bonardi O. (a cura di), 
Logistica e lavoro, Quaderni Riv. Giur. Lav., Roma, Ediesse, 2018, p. 127 ss.

GAETA L., «La terza dimensione del diritto»: legge e contratto collettivo nel Novecento italiano, in AA.VV., Legge e contrattazione collettiva nel diritto del lavoro post-statutario. Atti delle giornate di studio di diritto del lavoro. Napoli, 16-17 giugno 2016, Milano, Giuffrè, 2017, p. $11 \mathrm{ss}$.

Galgano F., Delle associazioni non riconosciute e dei comitati. Art. 36-42, Bologna-Roma, Zanichelli-Società editrice del Foro italiano, 1967.

GalizIA C., La giusta retribuzione tra punti fermi e questioni aperte, in Dir. Lav. Merc., 2009, p. 597 ss.

Garilli A., Categorie dei lavoratori (in generale), in Dig. Comm., III, 1988, p. 40 ss.

GarILLI A., Intervento, in Autonomia collettiva e occupazione. Atti del XII Congresso nazionale di diritto del lavoro Milano, 23-25 maggio 1997, Milano, Giuffrè, 1997, p. 139 ss.

Garofalo D., Il lavoro a domicilio, in Riv. It. Dir. Lav., 2018, III, p. 131 ss. Garofalo D., Il lavoro nel terzo settore, in Mass. Giur. Lav., 2018, p. 91 ss. Garofalo M.G., Attività creatrice del giudice e ricerca sui fatti, in Pol. Dir., 1972, p. 264 ss.

GAROFALO M.G., Interessi collettivi e comportamento antisindacale dell'imprenditore, Napoli, Jovene, 1979.

Garofalo M.G., Decentramento produttivo, impresa-rete e area contrattuale dei bancari, in Riv. Giur. Lav., 1991, I, p. 71 ss.

Garofalo M.G., Per una teoria giuridica del contratto collettivo. Qualche osservazione di metodo, in Dir. Lav. Rel. Ind., 2011, p. 515 ss.

Garofalo M.G., Solidarietà, differenze, riunificazione del mondo del lavoro (1991), in Barbieri M., Voza R. (a cura di), Gianni Garofalo. Il pane del sapere, Roma, Ediesse, 2011, p. 295 ss.

GHera E., Libertà sindacale e ordinamento della polizia, in Giur. Cost., 1976, p. 656 ss.

GHERA E., Le c.d. clausole sociali: evoluzione di un modello di politica legislativa, in Dir. Rel. Ind., 2001, p. 133 ss.

GHEZzi G., La responsabilità contrattuale delle associazioni sindacali, Milano, Giuffrè, 1963.

GHEzzi G., L'interpretazione delle norme delegate sul trattamento minimo dei prestatori di lavoro, in Riv. Trim. Dir. Proc. Civ., 1963, p. 339 ss.

Ghezzi G., L'intervento del giudice nel conflitto industriale, in Dir. Lav. Rel. Ind., 1983, p. 489 ss. 
GIUGNI G., Bargaining units and labor organization in Italy, in Ind. Lab. Rel. Rev., 1957, 10, 3, p. 424 ss.

GiUgNI G., La disciplina legislativa del trattamento minimo di categoria, in Riv. Trim. Dir. Proc. Civ., 1959, p. 863 ss.

GiUGNI G., Introduzione allo studio dell'autonomia collettiva, Milano, Giuffrè, 1960.

Giugni G., La validità "erga omnes" dei contratti collettivi, in Nord e Sud, 1960, 2, p. 62 ss.

Giugni G., Osservazioni sulle sentenze n. 70 e 106 della Corte costituzionale, in Giur. Cost., 1963, p. 822 ss.

Giugni G., La funzione giuridica del contratto collettivo di lavoro, in AA.VV., Il contratto collettivo di lavoro, Atti del III congresso nazionale dell'Aidlass, Milano, Giuffrè, 1968, p. 11 ss.

Giugni G., Lavoro giornalistico, in Enc. Dir., XXIII, 1973, p. 448 ss.

Giugni G., Art. 39, in Giu. Branca (a cura di), Commentario della Costituzione, Rapporti economici, I, Bologna-Roma, Zanichelli-Società editrice del Foro italiano, 1979, p. 257 ss.

Giugni G., Introduzione a Perlman S., Per una teoria dell'azione sindacale, Roma, Edizioni lavoro, 1980.

GiUgni G., Giuridificazione e deregolazione nel diritto del lavoro italiano, in Dir. Lav. Rel. Ind., 1986, p. 316 ss.

GiUgni G., Diritto del lavoro (voce per un'enciclopedia), in GIUGNI G., Lavoro legge contratti, Bologna, Il Mulino, 1989, p. 245 ss.

GiUgni G., Libertà sindacale, in Dig. Comm., IX, 1993, p. 17 ss.

GIUGNI G., Intervento, in Autonomia collettiva e occupazione. Atti del XII Congresso nazionale di diritto del lavoro Milano, 23-25 maggio 1997, Milano, Giuffrè, 1997, p. 239 ss.

Giugni G., Diritto sindacale, con la collaborazione di Bellardi L., Curzio P., Garofalo M.G., Bari, Cacucci, 2008.

GotTARDi D., Significato e anomalia di un contratto, in Lav. Inf., 1997, p. 21 ss.

GotTARDi D., La contrattazione collettiva tra destrutturazione e ri-regolazione, in Lav. Dir., 2016, p. 877 ss.

Gragnoli E., Art. 39, in Grandi M., Pera G., Commentario breve alle leggi sul lavoro, Padova, Cedam, 1996, p. 76 ss.

Gragnoli E., Profili dell'interpretazione dei contratti collettivi, Milano, Giuffrè, 2000.

Gragnoli E., Sciopero nei servizi pubblici essenziali, in Dig. Comm., Agg., 2003, p. 768 ss. 
Gragnoli E., La parabola del contratto collettivo nella società economica italiana, in Lav. Giur., 2013, p. 653 ss.

Gragnoli E., Esiste ancora un ordinamento intersindacale?, in AA.VV., Studi in memoria di Mario Giovanni Garofalo, 1, Bari, Cacucci, 2015, p. 433 ss.

Gragnol E., L'interpretazione di un contratto collettivo corporativo e il regime previdenziale dei produttori assicurativi, in Dir. Rel. Ind., 2017, p. 814 ss.

Grandi M., I problemi del lavoro negli orientamenti della dottrina giuridica: organizzazione sindacale, contratto collettivo e sciopero, in ZANINELLI S. (a cura di), Il sindacato nuovo. Politica ed organizzazione del movimento sindacale in Italia negli anni 1943-1955, Milano, Franco Angeli, 1981, p. 185 ss.

GRANDI M., Il problema della maggiore rappresentatività sindacale davanti alla Corte costituzionale (nella questione dei Sinquadri), in Riv. It. Dir. Lav., 1989, I, p. 141 ss.

Grandi M., Normatività privata e contratto collettivo, in AA.VV., Il sistema delle fonti nel diritto del lavoro. Atti delle giornate di studio di diritto del lavoro Foggia-Baia delle Zagare, 25-26 maggio 2001, Milano, Giuffrè, 2002, p. 216 ss.

Grandi M., In difesa della rappresentanza sindacale, in Dir. Lav. Rel. Ind., 2004, p. 627 ss.

Greco M.G., Contrattazione collettiva, contratti pirata e regolamenti nella determinazione dei livelli retributivi nelle cooperative di produzione e lavoro, in Variaz. Temi Dir. Lav., 2017, p. 367 ss.

Guarriello F., Crisi economica, contrattazione collettiva e ruolo della legge, in Dir. Lav. Rel. Ind., 2016, p. 3 ss.

GuerRIERI D., La categoria nell'ordinamento giuridico del lavoro, Milano, Giuffrè, 1963.

GUERRIERI D., I vigenti decreti di applicabilità del contratto collettivo, in Mass. Giur. Lav., 1969, p. 383 ss.

Hayter S., Visser J. (eds), Collective Agreements: Extending Labour Protection, Geneva, International Labour Organization, 2018.

Hayter S., Visser J., The application and extension of collective agreements: Enhancing the inclusiveness of labour protection, in HAYTER S., Visser J. (eds), Collective Agreements: Extending Labour Protection, Geneva, International Labour Organization, 2018, p. $1 \mathrm{ss}$.

Ichino P., Funzione ed efficacia del contratto collettivo nell'attuale sistema 
delle relazioni sindacali e nell'ordinamento statale, in Riv. Giur. Lav., 1975, I, p. 457 ss.

Ichino P., Note in materia di estensione dell'efficacia del contratto collettivo, in Riv. Giur. Lav., 1978, II, p. 930 ss.

Ichino P., I primi due decenni del diritto del lavoro repubblicano, I. Dalla liberazione alla metà degli anni '50, II. Dalla metà degli anni '50 alla legge sui licenziamenti individuali, in Riv. It. Dir. Lav., 2007, I, pp. 221 ss., 249 ss.

Ichino P., La crepa interna dell'articolo 39 della Costituzione, in www.pietroichino.it.

Imberti L., Il socio lavoratore di cooperativa. Disciplina giuridica ed evidenze empiriche, Milano, Giuffrè, 2012.

Imberti L., Art. 36 Costituzione: in assenza di interventi legislativi chi è l'autorità salariale?, in Lav. Dir. Eur., 3, 2019.

Imberti L., Trattamento economico minimo (del socio lavoratore) e c.c.n.l. parametro: chi individua la categoria ed il perimetro della stessa?, in Labor, 2019, p. 404 ss.

Istat, Classificazione delle attività economiche Ateco 2007, Roma, Istituto nazionale di statistica, 2009.

Istat, La classificazione delle professioni, Roma, Istituto nazionale di statistica, 2013.

Izzi D., Lavoro negli appalti e dumping salariale, Torino, Giappichelli, 2018.

Jacobs A., Labour law in the Netherlands, II ed., Alphen aan den Rijn, Kluwer law international, 2015.

Jemolo A.C., Ancora sui concetti giuridici, in Riv. Dir. Comm., 1945, I, p. $130 \mathrm{ss}$.

Kahn-Freund O., Intergroup Conflicts and Their Settlement, in Brit. J. Soc., 1954, 5, 3, p. 193 ss.

Lambertucci P., Area contrattuale e autonomia collettiva, in Dir. Lav. Rel. Ind., 1999, p. 277 ss.

Lambertucci P., Contratto collettivo, rappresentanza e rappresentatività sindacale: spunti per il dibattito, in Dir. Lav. Rel. Ind., 2009, p. 551 ss.

LAmbertucci P., Le linee evolutive della libertà di associazione sindacale per i militari, in Lav. Dir., 2019, p. 305 ss.

LASSANDARI A., Intervento, in AA.VV., Autonomia collettiva e occupazione. Atti del XII Congresso nazionale di diritto del lavoro Milano, 2325 maggio 1997, Milano, Giuffrè, 1997, p. 202 ss. 
Lassandari A., Pluralità di contratti collettivi nazionali per la medesima categoria, in Lav. Dir., 1997, p. 261 ss.

LASSANDARI A., Il contratto collettivo aziendale e decentrato, Milano, Giuffrè, 2001.

Lassandari A., Campo di applicazione del contratto, in Garofalo M.G., RocCELla M. (a cura di), Commentario al contratto collettivo nazionale di lavoro dei metalmeccanici: 20 gennaio 2008, Bari, Cacucci, 2010, p. 29 ss.

LASSANDARI A., La contrattazione collettiva: prove di de-costruzione di un sistema, in Lav. Dir., 2011, p. 321 ss.

LASSANDARI A., Tre proposte per una legge sindacale, in AA.VV., L'attuazione degli articoli 39 e 46 della Costituzione. Tre proposte a confronto, Quaderni Riv. Giur. Lav., Roma, Ediesse, 2016, p. 37 ss.

LASSANDARI A., Sulla verifica di rappresentatività delle organizzazioni sindacali datoriali, in Dir Lav. Rel. Ind., 2017, p. 1 ss.

Lassandari A., Retribuzione e contrattazione collettiva, in Riv. Giur. Lav., 2019, I, p. 210 ss.

LAZZARI C., Rappresentanza e rappresentatività sindacale nel lavoro pubblico, in Prola G. (a cura di), Organizzazione sindacale e contrattazione collettiva, in CARINCI F., Persiani M. (dir. da), Trattato di diritto del lavoro, Padova, Cedam, 2014, p. 1081 ss.

LAZZERONI L., La regolazione per legge del contratto collettivo aziendale alla luce del sistema costituzionale, Torino, Giappichelli, 2017.

Leccese V., Il diritto sindacale al tempo della crisi. Intervento eteronomo e profili di legittimità costituzionale, in Dir. Lav. Rel. Ind., 2012, p. 479 ss.

LeCCESE V., Contrattazione a efficacia generale e sistema contrattuale: le proposte di legge Cgil, «Diritti lavori mercati» e «Freccia Rossa», in AA.VV., L'attuazione degli articoli 39 e 46 della Costituzione. Tre proposte a confronto, Quaderni Riv. Giur. Lav., Roma, Ediesse, 2016, p. 75 ss.

LECCESE V., La contrattazione collettiva nazionale oggi: caratteri, metamorfosi e criticità. Temi per il dibattito, in Lassandari A., MarTelloNi F., Tuluini P., Zoli C. (a cura di), La contrattazione collettiva nello spazio economico globale, Bologna, Bononia University Press, 2017, p. 109 ss.

Leccese V., Pinto V., Osservazioni in tema di lavoro temporaneo, in GHera E. (a cura di), Occupazione e flessibilità. Legge n. 196/1997 e provvedimenti attuativi, Napoli, Jovene, 1998, p. 1 ss.

Leccese V., Roma G., Lozito M., La contrattazione nazionale di categoria, 
in Mascini M. (a cura di), l'Annuario del lavoro 2018, Roma, Il diario del lavoro, 2018, p. 77 ss.

LeCcese V., Roma G., Lozito M., La contrattazione nazionale di categoria, in Mascini M. (a cura di), l'Annuario del lavoro 2019, Roma, Il diario del lavoro, 2019, p. 67 ss.

Liebman S., Contributo allo studio della contrattazione collettiva nell'ordinamento giuridico italiano, Milano, Giuffrè, 1986.

Liebman S., Autonomia collettiva e legge, in D'Antona M. (a cura di), Letture di diritto sindacale, Napoli, Jovene, 1990, p. 49 ss.

Linee guida di una proposta di "Legge sindacale", in Riv. It. Dir. Lav., 2015, III, p. 207 ss.

Liso F., Categorie e qualifiche del lavoratore, in Enc. Giur. Trecc., VI, 1988, p. 1 ss.

Liso F., Autonomia collettiva e occupazione, in Dir. Lav. Rel. Ind., 1998, p. 191 ss.

Liso F., Brevi note sul protocollo Confindustria, Cgil, Cisl e Uil del maggio 2013, in Riv. Giur. Lav., 2013, I, p. 837 ss.

Liso F., Qualche erratica considerazione sul recente accordo interconfederale Confindustria, Cgil, Cisl e Uil del 9 marzo 2018, in Boll. Adapt, 23 aprile 2018, n. 16.

Lo Faro A., Teorie autopoietiche e diritto sindacale, in Lav. Dir., 1993, p. 129 ss.

Lo Faro A., Contratto collettivo (lavoro privato), in Enc. Dir., Annali, VI, 2013, p. 196 ss.

Loffredo A., La Commissione di garanzia: un'autorità indipendente tra diritto amministrativo e ordinamento intersindacale, in Lav. Dir., 2005, p. 545 ss.

LUNARDON F., Rappresentatività sindacale e categorie professionali, in AA.VV., La rappresentatività del sindacato, Quaderni di diritto del lavoro e delle relazioni industriali, Torino, Utet, 1989, p. 75 ss.

Lunardon F. (a cura di), Conflitto, concertazione e partecipazione, in CArinci F., Persiani M. (dir. da), Trattato di diritto del lavoro, Padova, Cedam, 2011.

MacKenzie R., Martinez Lucio M., The Realities of Regulatory Change: Beyond the Fetish of Deregulation, in Sociology, 2005, p. 499 ss.

Magnani M., La disciplina dello sciopero nei servizi pubblici essenziali alla prova dei fatti, in Riv. It. Dir. Lav., 2005, I, p. 69 ss.

Magnani M. (a cura di), Diritto di sciopero e assetto costituzionale, Milano, Giuffrè, 2010. 
Magnani M., Il salario minimo legale, in Riv. It. Dir. Lav., 2010, I, p. 769 ss.

Magnani M., Rappresentatività e diritti sindacali tra autonomia collettiva e giustizia costituzionale, in CARINCI F. (a cura di), Legge o contrattazione? Una risposta sulla rappresentanza sindacale a Corte costituzionale n. 231/2013, Adapt Labour Studies, E-Book Series, n. 20, 2014, p. 47 ss.

Magnani M., Diritto sindacale europeo e comparato, Torino, Giappichelli, 2015.

Magnani M., Riflessioni sulla misurazione della rappresentanza datoriale nell'ordinamento statale e intersindacale, in WP CSDLE "Massimo D'Antona".IT, 376/2018.

Magnani M., I tempi e i luoghi del lavoro. L'uniformità non si addice al post-fordismo, in WP CSDLE "Massimo D'Antona”.IT, 404/2019.

MaINARDi S., Il potere disciplinare nel lavoro privato e pubblico, Milano, Giuffrè, 2002.

MaINARDi S., Fonti, poteri e responsabilità nella valutazione del merito dei dipendenti pubblici, in Lav. Pubb. Amm., 2009, p. 729 ss.

MaINARDi S., Le relazioni collettive nel "nuovo" diritto del lavoro, in AA.VV., Legge e contrattazione collettiva nel diritto del lavoro poststatutario. Atti delle giornate di studio di diritto del lavoro. Napoli, 1617 giugno 2016, Milano, Giuffrè, 2017, p. 181 ss.

Marolini F., Rapporti di partecipazione societaria ed ambito di applicazione del contratto collettivo nel sistema creditizio, in Dir. Rel. Ind., 1993, p. 39 ss.

Mancini G.F., La rappresentanza sindacale nel diritto statunitense e l'art. 39 della Costituzione italiana, in Riv. Dir. Lav., 1950, I, p. 463 ss.

MANCINI G.F., Libertà sindacale e contratto collettivo "erga omnes", in Riv. Trim. Dir. Proc. Civ., 1963, p. 570 ss.

Mancini G.F., I sindacati: fabbrica e riforme, in Pol. Dir., 1971, p. 211 ss.

Mancini G.F., Art. 36, in Ghezzi G., Mancini G.F., Montuschi L., Romagnoli U., Statuto dei lavoratori, in Scialoja A., Branca Giu. (a cura di), Commentario del codice civile, Bologna-Roma, Zanichelli-Soc. ed. del Foro italiano, 1972, p. 542 ss.

Mancini G.F., Lo statuto dei lavoratori dopo le lotte operaie del 1969 (1970), in Mancini G.F., Costituzione e movimento operaio, Bologna, Il Mulino, 1976, p. 133 ss.

Mancini G.F., Sindacato e Costituzione trent'anni dopo, in Mancini G.F., 
Costituzione e movimento operaio, Bologna, Il Mulino, 1976, p. 163 sS.

Maranini G., Marzotto A., L'art. 39 della Costituzione e l'organizzazione pubblica della categoria professionale, in Riv. Dir. Lav., 1949, I, p. 181 ss.

MarazZa M., L'artigianato, in Mascini M. (a cura di), l'Annuario del lavoro 2010, Viterbo, Union Printing, 2010, p. 247 ss.

Marazza M., Dalla "autoregolamentazione" alla "legge sindacale"? La questione dell'ambito di misurazione della rappresentatività sindacale, in Arg. Dir. Lav., 2014, p. 608 ss.

Marazza M., Perimetri e rappresentanze sindacali (dei datori di lavoro e dei lavoratori), in Lab. Law Iss., 2018, 2, p. III ss.

Maresca A., Accordi collettivi separati: tra libertà contrattuale e democrazia sindacale, in Riv. It. Dir. Lav., 2010, I, p. 29 ss.

Maresca A., Il contratto collettivo nazionale di categoria dopo il Protocollo d'intesa 31 maggio 2013, in Riv. It. Dir. Lav., 2013, I, p. 707 ss.

Maresca A., Il rinnov(ament)o del contratto collettivo dei meccanici: c'è ancora un futuro per il contratto collettivo nazionale di categoria, in Dir. Lav. Rel. Ind., 2017, p. 709 ss.

Marginson P., Industrial Relations at European Sector Level: The Weak Link?, in Eco. Ind. Dem., 2005, 4, p. 511 ss.

MARIUCCI L., Il lavoro decentrato: discipline legislative e contrattuali, Milano, Franco Angeli, 1979.

Mariucci L., La contrattazione collettiva, Bologna, Il Mulino, 1985.

Mariucci L., Uno sguardo alle relazioni industriali negli USA, in Lav. Dir., 1987, p. 75 ss.

MARIUCCI L., Gli eterni ritorni: dentro, fuori o oltre l'art. 39 della Costituzione?, in Zoppoli L., Zoppoli A., Delfino M. (a cura di), Una nuova Costituzione per il sistema di relazioni sindacali?, Napoli, Editoriale scientifica, 2014, p. 487 ss.

Mariucci L., L'attualità di un dibattito antico. Introduzione a L'attuazione degli articoli 39 e 46 della Costituzione. Tre proposte a confronto, Quaderni Riv. Giur. Lav., Roma, Ediesse, 2016, p. 9 ss.

Martelloni F., Processo al sindacato, trent'anni dopo, in Lassandari A., Martelloni F. (a cura di), Giorgio Ghezzi. Processo al sindacato, Roma, Ediesse, 2012, p. 147 ss.

Martelloni F., Presenza nel conflitto e rappresentatività nell'interregno del sistema sindacale, in Lav. Dir., 2014, p. 55 ss.

MASCINI M., Le relazioni industriali, in MASCINI M. (a cura di), l'Annuario del lavoro 2017, Roma, Il diario del lavoro, 2017, p. 25 ss. 
Mascini M., Le relazioni industriali, in MAscini M. (a cura di), l'Annuario del lavoro 2018, Roma, Il diario del lavoro, 2018, p. 23 ss.

Mazzoni G., La conquista della libertà sindacale, Roma, Edizioni Leonardo, 1947.

Mazzoni G., I limiti di applicabilità dell'art. 2070 cod. civ. ed il potere sindacale di valutazione degli interessi collettivi, in Mass. Giur. Lav., 1964, p. 107 ss.

Mazzoni G., L'azione sindacale e lo Statuto dei lavoratori, Milano, Giuffrè, 1974.

Mazzotta O., Intervento, in AA.VV., Categorie professionali e contratti collettivi, Suppl. Notiz. Giur. Lav., Roma, Assicredito, 1990, p. 97 ss.

Mazzotтa O., Legge e contratto collettivo nelle trasformazioni delle imprese, in AA.VV., Le trasformazioni aziendali in vista del Mercato europeo: legge e contratto collettivo, Roma, Bancaria, 1992, p. 46 ss.

Mazzotta O., Divagazioni in tema di interpretazione del contratto collettivo, in Flammia R. (a cura di), L'interpretazione dei contratti collettivi di lavoro, Roma, Bancaria, 1999, p. 75 ss.

Mazzotta O., Il diritto del lavoro e le sue fonti, in Riv. It. Dir. Lav., 2001, I, p. 219 ss.

Mengoni L., Le fonti del diritto del lavoro in Italia, in G. Boldt et al., Le fonti del diritto del lavoro, Lussemburgo, Comunità europea del carbone e dell'acciaio Alta autorità, 1962, p. 135 ss.

Mengoni L., Il regime giuridico delle organizzazioni professionali in Italia, in BoldT G. et al., Il regime giuridico delle organizzazioni professionali nei Paesi membri della C.E.C.A., Lussemburgo, Comunità europea del carbone e dell'acciaio Alta autorità, 1966, p. 375 ss.

Mengoni L., La questione del «diritto giusto» nella società post-liberale, in Rel Ind., 1988, 11, p. 13 ss.

Mercader Uguina J. R., Art. 83. Unidades de negociación, in Villalon J. C. et al., Comentarios al estatuto de los trabajadores, IV ed., Madrid, Lex Nova-Thomson Reuters, 2016, p. 990 ss.

Modugno F., Norma giuridica (teoria generale), in Enc. Dir., XXVIII, 1978, p. 328 ss.

Momigliano F., Sindacati, progresso tecnico, programmazione economica, Torino, Einaudi, 1966.

Monateri P. G., Fattispecie, in Dig. Civ., VIII, 1992, p. 224 ss.

Montuschi L., I limiti legali nella conclusione del contratto di lavoro, Milano, Giuffrè, 1964.

Montuschi L., La Costituzione e i lavori, in Riv. It. Dir. Lav., 2009, I, p. 153 ss. 
NADAL S., La restructuration des branches professionnelles: réflexions sur une mutation forcée, in Dr. Soc., 2016, p. 110 ss.

NADAL S., Gouvernance du niveau et des règles de branche: les nouveaux visages de l'emprise étatique, in Rev. Dr. Trav., 2017, p. 652 ss.

NAPOLI M., I sindacati maggiormente rappresentativi: rigorosità del modello legislativo e tendenze della prassi applicativa, in AA.VV., La rappresentatività del sindacato, Quaderni di diritto del lavoro e delle relazioni industriali, Torino, Utet, 1989, p. 7 ss.

Napoli M., Il quadro giuridico-istituzionale, in Cella G.P., Treu T. (a cura di), Le nuove relazioni industriali. L'esperienza italiana nella prospettiva europea, Bologna, Il Mulino, 1998, p. 47 ss.

NApoli M., Sindacato, in Dig. Comm., XVI, Appendice, 1999, p. 509 ss.

NAPOLI M., Autonomia collettiva e autonomia individuale nelle più recenti riforme, in Dir. Lav. Rel. Ind., 2004, p. 581 ss.

Natoli U., Alcuni rilievi in vista dell'attuazione della legge 15 luglio 1959, n. 741, in Riv. Giur. Lav., 1959, I, p. 103 ss.

Natullo G., La nuova contrattazione collettiva nel lavoro pubblico: ambito e limiti, in Ist. Fed., 2009, p. 685 ss.

Nicolosi M., Libertà sindacale separata e Polizia di Stato, in Lav. Giur., 2012, p. 1051 ss.

Nieto Rojas P., La respuesta de la negociacion colectiva sectorial a las empresas multiservicios, in Riv. Inf. Lab., 2017, 7, p. 229 ss.

Nogler L., Lavoro a domicilio. Art. 2128, Milano, Giuffrè, 2000.

Nogler L., La disciplina dei licenziamenti individuali nell'epoca del bilanciamento tra i "principi" costituzionali, in Dir. Lav. Rel. Ind., 2007, p. 593 ss.

Nogler L., Brun S., Art. 36, in De Luca Tamajo R., Mazzotta O. (a cura di), Commentario breve alle leggi sul lavoro, VI ed., Milano, Wolters Kluwer-Cedam, 2018, p. 40 ss.

Novella M., Applicazione del contratto collettivo e categoria professionale. Vecchi e nuovi problemi, in Riv. Giur. Lav., 1999, I, p. 357 ss.

OecD, Employment Outlook 2017, Paris, Oecd publishing, 2017.

OuIN G., I contratti nazionali: quanti sono e perché crescono, in Dir. Lav. Rel. Ind., 2016, p. 417 ss.

Orlandini G., Clausole sociali (dir. lav. interno), in Treccani - diritto on line, 2015.

Orlandini G., Conflitto collettivo, in Enc. Dir., Annali, IX, 2016, p. 95 ss.

ORlandini G., Legge, contrattazione collettiva e giusta retribuzione, in Lav. Dir., 2018, p. 7 ss. 
Orsi Battaglini A., Della quadratura di molti circoli, in Pol. Dir., 1984, p. 3 ss.

Orsi Battaglini A., Relazioni sindacali e fonti normative nella legge quadro sul pubblico impiego, in Orsi BatTAGLINI A. et al., Accordi sindacali e legge quadro sul pubblico impiego, Milano, Giuffrè, 1984, p. 3 ss.

PAPA V., L'attività sindacale delle organizzazioni datoriali. Rappresentan$z a$, rappresentatività e contrattazione, Torino, Giappichelli, 2017.

PAPA V., Verso una rappresentanza misurata? Strumenti e metodi di misurazione della rappresentatività datoriale, in Dir. Lav. Rel. Ind., 2017, p. 21 ss.

Pascucci P., Tecniche regolative dello sciopero nei servizi essenziali, Torino, Giappichelli, 1999.

Pascucci P., Giusta retribuzione e contratti di lavoro, oggi, in Dir. Lav. Rel. Ind., 2019, p. 59 ss.

Passalacoua P., Autonomia collettiva e mercato del lavoro: la contrattazione gestionale e di rinvio, Torino, Giappichelli, 2005.

Passalacoua P., Il modello del sindacato comparativamente più rappresentativo nell'evoluzione delle relazioni sindacali, in Dir. Rel. Ind., 2014, p. 378 ss.

Pedrazzoli M., Alle origini del dibattito giuridico-istituzionale sulla democrazia industriale: il caso italiano, in AA.VV., Studi in onore di Tito Carnacini, I, Studi di diritto costituzionale, diritto civile, diritto del lavoro, diritto commerciale, Milano, Giuffrè, 1983, p. 791 ss.

Pedrazzoli M., Democrazia industriale e subordinazione. Poteri e fattispecie nel sistema giuridico del lavoro, Milano, Giuffrè, 1985.

Pedrazzoli M., Qualificazioni dell'autonomia collettiva e procedimento applicativo del giudice, in Lav. Dir., 1990, pp. 355 ss., 549 ss.

Penelope N., La contrattazione interconfederale, in Mascini M. (a cura di), l'Annuario del lavoro 2017, Roma, Il diario del lavoro, 2017, p. 75 ss.

Pera G., Fondamento ed efficacia del contratto collettivo di diritto comune (1958), in Scritti di Giuseppe Pera, II, Diritto sindacale, Milano, Giuffrè, 2007, p. 989 ss.

Pera G., L'obbligatorietà dei contratti collettivi di lavoro, in Il Ponte, 1959, p. 32 ss.

Pera G., Problemi costituzionali del diritto sindacale italiano, Milano, Feltrinelli, 1960.

Pera G., Lo sciopero di solidarietà nelle più recenti concezioni, in Riv. Giur. Lav., 1964, I, p. 1 ss. 
Pera G., Libertà sindacale (diritto vigente), in Enc. Dir., XXIV, 1974, p. 501 ss.

Pera G., Il trentanovismo è nelle cose, in Pol. Dir., 1985, p. 503 ss.

Pera G., La contrattazione collettiva di diritto comune e l'art. 2070 c.c., in Giust. Civ., 1997, p. 1203 ss.

Pera G., Note sui contratti collettivi «pirata», in Riv. It. Dir. Lav., 1997, I, p. 381 ss.

Pera G., Il rispetto della contrattazione collettiva nelle concessioni di pubblici servizi, in Giust. Civ., 1998, p. 2426 ss.

Pera G., La contrattazione collettiva a dominio confederale per il pubblico impiego, in Riv. It. Dir. Lav., 1998, II, p. 39 ss.

Persiani M., I soggetti del contratto collettivo con efficacia generale, in Dir. Lav., 1958, I, p. 88 ss.

Persiani M., Saggio sull'autonomia privata collettiva, Padova, Cedam, 1972.

Persiani M., Autonomia collettiva e retribuzione imponibile, in Mass. Giur. Lav., 1996, p. 670 ss.

PERSIANI M., La retribuzione tra legge, autonomia collettiva e determinazione giudiziale, in AA.VV., Nuove forme di retribuzione e attualità dei principi costituzionali. Atti dell'incontro di studio Roma, 3 febbraio 1997, Quaderni Arg. Dir. Lav., Padova, Cedam, 1998, p. 31 ss.

Persiani M., Il contratto collettivo di diritto comune nel sistema delle fonti del diritto del lavoro, in Arg. Dir. Lav., 2004, p. 13 ss.

PeRsiani M., Le vicende della rappresentanza e rappresentatività sindacali tra legge e contratto collettivo, in Arg. Dir. Lav., 2017, p. 531 ss.

Perskine E., Wolmark C., Droit du travail: 2019, Paris, Dalloz, 2018.

Perulli A., L'attività sindacale nella piccola impresa, in AA.VV., Piccola impresa e diritto del lavoro, Quaderni di diritto del lavoro e delle relazioni industriali, Torino, Utet, 1991, p. 163 ss.

Perulli A., I rinvii all'autonomia collettiva: mercato del lavoro e trasferimento d'azienda, in Dir. Lav. Rel. Ind., 1992, p. 533 ss.

Perulli A., La nuova definizione di collaborazione organizzata dal committente, in Riv. It. Dir. Lav., 2019, III, p. 163 ss.

Pessi R., Ripensando alla riforma della pubblica amministrazione: manutenzione o restaurazione?, in Mass. Giur. Lav., 2009, p. 284 ss.

Piglialarmi G., La rappresentatività tra libertà sindacale e categoria merceologica. A proposito di una recente riflessione di Pietro Ichino, in Boll. Adapt, 7 ottobre 2019, n. 35.

Pilati A., Il campo di applicazione della legge e i requisiti di legittimità del- 
le astensioni collettive, in LUNARDON F. (a cura di), Conflitto, concertazione e partecipazione, in Carinci F., Persiani M. (dir. da), Trattato di diritto del lavoro, Padova, Cedam, 2011, p. 277 ss.

PIno G., Conflitto collettivo nei servizi pubblici essenziali, in Enc. Dir., Annali, X, 2017, p. 265 ss.

PINTO V., Gli accordi interconfederali del 2013 e i persistenti problemi teorici (e pratici) della definizione autonoma della "categoria contrattuale”, in Barbera M., Perulli A. (a cura di), Consenso, dissenso e rappresentanza: le nuove relazioni sindacali, Cedam, Padova, 2014, p. 361 ss.

Pizzoferrato A., Gli interventi sull'art. 2070 c.c., in Zoli C. (a cura di), Le fonti. Il diritto sindacale, in CARINCI F. (dir. da), Diritto del lavoro. Commentario, II ed., Torino, Utet, 2007, p. 374 ss.

Pizzoferrato A., L'autonomia collettiva nel nuovo diritto del lavoro, in Dir. Lav. Rel. Ind., 2015, p. 411 ss.

Poisson J.-F., Rapport sur la négociation collective et les branches professionnelles, Rapport au Premier ministre, Paris, La documentation française, Remis le 28 avril 2009.

PORTA J., La branche professionnelle: déconstruction et restructuration, in Dr. Ouvr., 2018, 842, p. 1 ss.

Proia G., L'inquadramento dei datori di lavoro, Milano, Giuffrè, 1993.

Proia G., Ostacoli ed incognite per l'intervento legislativo sulla rappresentanza sindacale, in Zoppoli L., Zoppoli A., Delfino M. (a cura di), Una nuova Costituzione per il sistema di relazioni sindacali?, Napoli, Editoriale Scientifica, 2014, p. 507 ss.

Proia G., Gambacciani M., Il contratto collettivo di diritto comune, in Proia G. (a cura di), Organizzazione sindacale e contrattazione collettiva, in Carinci F., Persiani M. (dir. da), Trattato di diritto del lavoro, Padova, Cedam, 2014, p. 595 ss.

Prosperetti G., La commissione di garanzia, in D'Onghia M., Ricci M. (a cura di), Lo sciopero nei servizi pubblici essenziali, Milano, Giuffrè, 2003, p. 43 ss.

Prosperetti U., Lezioni di diritto sindacale comparato, Milano, Giuffrè, 1962.

Prosperetti U., Libertà sindacale (premesse generali), in Enc. Dir., XXIV, 1974, p. 494 ss.

Quaranta M., Art. 2070, in De Luca Tamajo R., Mazzotta O. (a cura di), Commentario breve alle leggi sul lavoro, VI ed., Milano, Wolters Kluwer-Cedam, 2018, p. 497 ss. 
RatTI L., Autonomia collettiva e tutela dell'occupazione. Elementi per un inquadramento delle clausole di riassunzione nell'ordinamento multilivello, Milano, Wolters Kluwer-Cedam, 2018.

ReCCHIA G.A., I rinvii al contratto collettivo nel lavoro agile tra ambiguità normative e ragionevole implementazione dell'istituto, in Arg. Dir. Lav., 2018, p. 1501 ss.

Relazione annuale 2019 sull'attività svolta nell'anno 2018 della Commissione di garanzia.

Relazione del Presidente Giuseppe Santoro-Passarelli per l'anno 2018, Roma, 18 giugno 2019.

Ricci M., Autonomia collettiva e giustizia costituzionale, Bari, Cacucci, 1999.

Ricci M., Il contratto collettivo fonte e l'art. 39 Cost., in AA.VV., Il sistema delle fonti nel diritto del lavoro. Atti delle giornate di studio di diritto del lavoro Foggia-Baia delle Zagare, 25-26 maggio 2001, Milano, Giuffrè, 2002, p. 138 ss.

Ricci M., L'accordo interconfederale del 9 marzo 2018: una svolta dagli esiti incerti, in Arg. Dir. Lav., 2018, p. 1392 ss.

Ricciardi M., Riflessioni sull'entrata a regime del nuovo sistema di relazioni sindacali nel lavoro pubblico, in Dir. Rel. Ind., 2002, p. 611 ss.

RichaRd R., Lineamenti generali dell'attività di inquadramento sindacale, Pavia, Libreria internazionale Garzanti, 1942.

Risposte al questionario del Ministro del lavoro e della previdenza sociale sul tema Verso la nuova legislazione sindacale, in Riv. Dir. Lav., 1949, III, p. 94 ss.

Riva Sanseverino L., Il contratto collettivo nella legislazione italiana, in Dir. Lav., 1946, I, p. 3 ss.

Riva Sanseverino L., Della impresa: disciplina delle attività professionali. Impresa in generale, II ed., Bologna-Roma, Zanichelli-Società editrice del Foro italiano, 1956.

Riva Sanseverino L., Contratto collettivo di lavoro, in Enc. Dir., X, 1962, p. 55 ss.

Riva Sanseverino L., Corporazione (diritto corporativo), in Enc. Dir., X, 1962, p. 679 ss.

Rizzuto G., Tomassetti P., Il dumping contrattuale nel settore moda, Roma, Edizioni lavoro, 2019.

Roccella M., Contrattazione collettiva, azione sindacale, problemi di regolazione del mercato del lavoro, in Lav. Dir., 2000, p. 351 ss.

Romagnoli U., Politica e tecnica nella contrattazione collettiva, in Riv. Trim. Dir. Proc. Civ., 1962, p. 245 ss. 
Romagnoli U., Il contratto collettivo d'impresa, Milano, Giuffrè, 1963.

Romagnoli U., Il contratto collettivo difficile (1971), in Romagnoli U., Lavoratori e sindacati tra vecchio e nuovo diritto, Bologna, Il Mulino, 1974, p. 213 ss.

Romagnoli U., La resistibile ascesa del contrattualismo nel pubblico impiego, in Dir Lav. Rel. Ind., 1980, p. 182 ss.

Romagnoli U., "Noi e loro»: diritto del lavoro e nuove tecnologie, in Riv. Trim. Dir. Proc. Civ., 1986, p. 377 ss.

Romagnoli U., L'inutile necessità di una disputa, in Dir. Lav. Rel. Ind., 1996, p. 1 ss.

Romagnoli U., Il contratto collettivo, in Dir. Lav. Rel. Ind., 2000, p. 225 SS.

Romagnoli U., Ballestrero M.V., Art. 40 Supplemento: Legge 12 giugno 1990 n. 146. Norme sull'esercizio del diritto di sciopero nei servizi pubblici essenziali, in Branca Giu. (a cura di), Commentario della Costituzione, Bologna-Roma, Zanichelli-Società editrice del Foro italiano, 1994.

Romer R., Di che cosa parliamo quando parliamo di sciopero, in Lav. Dir., 1999, p. 251 ss.

Rucco G., Commentario al d.lgs. 27 ottobre 2009, n. 150, Roma, SSPAL, 5 luglio 2010.

Rusciano M., In tema di disciplina del contratto collettivo di lavoro, in Mass. Giur. Lav., 1986, p. 423 ss.

Rusciano M., Il contratto collettivo, in Impresa e lavoro, T. I, II ed., in REscigno P. (dir. da), Trattato di diritto privato, Torino, Utet, 2004, p. $1 \mathrm{ss}$.

Rusciano M., Diritto di sciopero e assetto costituzionale, in Riv. It. Dir. Lav., 2009, I, p. 59 ss.

Rusciano M., Introduzione. Che fare dell'art. 39 della Costituzione?, in Zoppoli L., Zoppoli A., Delfino M. (a cura di), Una nuova Costituzione per il sistema di relazioni sindacali?, Napoli, Editoriale Scientifica, 2014, p. 439 ss.

Rusciano M., Sciopero, servizi essenziali, moral suasion della commissione di garanzia, in AA.VV., Diritto di sciopero e rappresentatività sindacale, Milano, Wolters Kluwer-Cedam, 2018, p. 41 ss.

Russo C., Aspettando Godot? Nuovi comparti e aree dirigenziali in attesa del rinnovo 2016-2018, in Dir. Lav. Merc., 2016, p. 439 ss.

SAcco R., Fattispecie, in Dig. Civ., Aggiornamento, 2010, p. 594 ss.

SAcco R., Fatto giuridico, in Dig. Civ., Aggiornamento, 2010, p. 610 ss. 
SAGLIO J., La régulation de branche dans le système français de relations professionnelles, in AA.VV., Les relations sociales en Europe, Paris, Ministère du Travail et de l'Emploi, SES, 1990, p. 37 ss.

Salomone R., Verso un nuovo regime collettivo per l'auto?, in CARINCI F. (a cura di), Da Pomigliano a Mirafiori: la cronaca si fa storia, Milano, Ipsoa, 2012, p. 107 ss.

Salomone R., La procedura per la contrattazione, in Proia G. (a cura di), Organizzazione sindacale e contrattazione collettiva, in CARINCI F., Persiani M. (dir. da), Trattato di diritto del lavoro, Padova, Cedam, 2014, p. 1265 ss.

Salomone R., Rappresentatività sindacale, ambiti negoziali e procedure nella contrattazione collettiva del pubblico impiego, in Dir. Lav. Merc., 2014, p. 495 ss.

SANTONI F., Rappresentatività sindacale e conflitto nei servizi pubblici essenziali, in Riv. It. Dir. Lav., 2003, I, p. 319 ss.

Santoni F., Contrattazione collettiva e principio di maggioranza, in Riv. It. Dir. Lav., 2013, I, p. 75 ss.

Santoro-Passarelli F., Intervento al I Congresso nazionale dei giuristi cattolici, in Iustitia, 1949.

Santoro-Passarelli F., Autonomia collettiva, giurisdizione, diritto di sciopero (1949) in Santoro-Passarelli F., Saggi di diritto civile, Napoli, Jovene, 1961, p. 177 ss.

Santoro-Passarelli F., L'autonomia dei privati nel diritto dell'economia (1956), in Santoro-Passarelli F., Saggi di diritto civile, Napoli, Jovene, 1961, p. 227 ss.

SAntoro-Passarelli F., La disciplina transitoria dei rapporti di lavoro, Roma, Edizioni Ricerche, 1961.

Santoro-Passarelli G., Sulla libertà sindacale dell'imprenditore, in Riv. Trim. Dir. Proc. Civ., 1976, p. 170 ss.

Santoro-Passarelli G., Art. 2, in Rusciano M., Santoro-Passarelli G. (a cura di), Lo sciopero nei servizi essenziali, Milano, Giuffrè, 1991, p. 19 ss.

Santoro-Passarelli G., Prospettive di riforma della rappresentanza sindacale nel lavoro privato, in Arg. Dir. Lav., 1999, p. 45 ss.

Santoro-Passarelli G., Sui lavoratori che operano mediante piattaforme anche digitali, sui riders e il ragionevole equilibrio della Cassazione 1663/2020, in WP CSDLE “Massimo D'Antona”.IT, 411/2020.

SANTUCCI R., Sistema di relazioni industriali e contratto collettivo, in SANtucci R., Zoppoli L. (a cura di), Contratto collettivo e disciplina dei rapporti di lavoro, II ed., Torino, Giappichelli, 2004, p. 11 ss. 
SANTUCCI R., Efficacia o inadeguatezza della disciplina dello sciopero nei servizi pubblici essenziali (in margine allo sciopero dei docenti universitari), in Arg. Dir. Lav., 2018, p. 662 ss.

Scarpelli F., Le fonti e il contenuto del principio di libertà sindacale, in Zoli C. (a cura di), Le fonti. Il diritto sindacale, in CARINCI F. (dir. da), Diritto del lavoro. Commentario, II ed., Torino, Utet, 2007, p. 65 ss.

SCARPELLI F., Linee e problemi dei fenomeni di esternalizzazione e decentramento produttivo, in Brollo M. (a cura di), Il mercato del lavoro, in Carinci F., Persiani M. (dir. da), Trattato di diritto del lavoro, Padova, Cedam, 2012, p. 1421 ss.

Scarpelli F., Il Testo Unico sulla rappresentanza tra relazioni industriali e diritto, in Dir. Rel. Ind., 2014, p. 687 ss.

SCARPelli F., Le tensioni del sistema di relazioni industriali e la riforma della rappresentanza, in AA.VV., L'attuazione degli articoli 39 e 46 della Costituzione. Tre proposte a confronto, Quaderni Riv. Giur. Lav., Roma, Ediesse, 2016, p. 167 ss.

SCARPoni S., Rappresentatività e organizzazione sindacale, Padova, Cedam, 2005.

Schiavo R., L'analisi congiunta dei Ccnl tra Cnel e Inps: un segnale d'urgenza di un dato sulla rappresentatività delle organizzazioni sindacali, in Boll. Adapt, 29 luglio 2019, n. 29.

Schmitter P.C., Still the Century of Corporatism?, in Rev. Pol., 1974, 36, 1 , p. 85 ss.

Schulten T., The role of extension in German collective bargaining, in HayTER S., VISSER J. (eds), Collective Agreements: Extending Labour Protection, Geneva, International Labour Organization, 2018, p. 65 ss.

Sciarra S., L'orgoglio dell'inattuazione costituzionale, in Pol. Dir., 1985, p. 421 ss.

Sciarra S., Contratto collettivo, in Dig. Comm., IV, 1989, p. 60 ss.

Scognamiglio R., Autonomia sindacale ed efficacia del contratto collettivo di lavoro, in Riv. Dir. Civ., 1971, p. 140 ss.

Scognamiglio R., A proposito di una recente iniziativa per una disciplina legislativa del contratto collettivo, in Mass. Giur. Lav., 1986, p. 683 ss.

Scognamiglo R., Intervento, in AA.VV., Categorie professionali e contratti collettivi, Suppl. Notiz. Giur. Lav., Roma, Assicredito, 1990, p. 129 ss.

Scognamiglio R., Il codice civile e il diritto del lavoro, in AA.VV., Le ragioni del diritto. Scritti in onore di Luigi Mengoni, II, Milano, Giuffrè, 1995, p. 1253 ss.

Scognamiglio R., Il problema dell'applicabilità dell'art. 2070 c.c.: catego- 
ria e contratto collettivo, in AA.VV., Scritti in onore di Giuseppe Federico Mancini, I, Diritto del lavoro, Milano, Giuffrè, 1998, p. 563 ss. SECHI I. (a cura di), Commissione Garanzia Sciopero, Raccolta sistematica degli orientamenti interpretativi. I, Orientamenti di carattere generale, 2016.

Sechi I., Conflitto d'accreditamento: ragioni ed evidenze empiriche di una patologia, in Dir. Lav. Rel. Ind., 2017, p. 605 ss.

Silvagna L., il sindacato comparativamente più rappresentativo, in Dir. Rel. Ind., 1999, p. 211 ss.

Simi V., Formazioni sociali e categoria professionale, in Riv. Trim. Dir. Proc. Civ., 1965, p. 436 ss.

Siotтo F., La categoria come ambito di applicazione e perimetro di misurazione della rappresentanza, in Riv. It. Dir. Lav., 2017, I, p. 311 ss.

Siotтo F., Ordinamento sindacale democratico. La prospettiva interna, Torino, Giappichelli, 2018.

SOLOPERTO R., La contrattazione collettiva nazionale e integrativa (artt. 36 e 53-56), in Tiraboschi M., Verbaro F. (a cura di), La nuova riforma del lavoro pubblico. Commento alla legge 4 marzo 2009, $n$. 15, e al decreto legislativo 27 ottobre 2009, n. 150, Milano, Giuffrè, 2010, p. 365 ss.

Speziale V., Contratto collettivo e lavoro pubblico: rapporti tra le fonti e principi costituzionali, in Dir. Lav. Rel. Ind., 1996, p. 307 ss.

Speziale V., Le politiche del lavoro del Governo Renzi: il Jobs Act e la riforma dei contratti e di altre discipline del rapporto di lavoro, in WP CSDLE “Massimo D'Antona”.IT, 233/2014.

Suppiej G., Pluralismo dei contratti collettivi e significato di un rinvio legislativo, in Riv. Dir. Lav., 1957, II, p. 212 ss.

Suppiej G., Fonti per lo studio del diritto sindacale, Padova, Cedam, 1965.

Suppiej G., La contrattazione collettiva tra crisi economica e riforme costituzionali, in Riv. It. Dir. Lav., 1986, I, p. 216 ss.

Talamo V., La contrattazione collettiva della dirigenza pubblica, in Giorn. Dir. Amm., 2004, p. 674 ss.

Talamo V., La riforma del sistema di relazioni sindacali nel lavoro pubblico, in Giorn. Dir. Amm., 2010, p. 13 ss.

Talamo V., Antefatti, luci ed ombre dei rinnovi contrattuali del pubblico impiego, in Giorn. Dir. Amm., 2018, p. 277 ss.

TARello G., Teorie e ideologie nel diritto sindacale, Milano, Edizioni di Comunità, 1967.

Tiraboschi M., Massagli E., La convenzione con INPS e INL, tra passi 
avanti e $i$ nodi politici della regolazione della rappresentanza che permangono, in Boll. Adapt, 23 settembre 2019, n. 33.

TOMASSETTI P., La nozione di sindacato comparativamente più rappresentativo nel decreto legislativo n. 81/2015, in Dir. Rel. Ind., 2016, p. 367 ss.

Tosi P., Contratto collettivo e rappresentanza sindacale, in Pol. Dir., 1985, p. 363 ss.

Tosi P., Le nuove tendenze del diritto del lavoro nel terziario, in Dir. Lav. Rel. Ind., 1991, p. 611 ss.

Tosi P., Gli assetti contrattuali fra tradizione e innovazione, in Arg. Dir. Lav., 2013, p. 506 ss.

Tosi P., Concorrenza, lavoro, diritti (aspetti collettivo-sindacali), in Arg. Dir. Lav., 2017, p. 1337 ss.

Tremolada M., Lavoro nelle cooperative, in Enc. Dir., Annali, VII, 2014, p. 627 ss.

Treu T., La CISL degli anni 50 e le ideologie giuridiche dominanti, in TARELlo G. (a cura di), Dottrine giuridiche e ideologie sindacali, Materiali per una storia della cultura giuridica, III/2, Bologna, Il Mulino, 1973, p. 269 ss.

TREU T. Il sindacato fuori dalla Costituzione: riconoscimento e rappresentatività, in Jus, 1975, p. 199 ss.

Treu T., Art. 36, in Branca Giu. (a cura di), Commentario della Costituzione. Rapporti Economici, I, Bologna-Roma, Zanichelli-Società editrice del Foro italiano, 1979, p. 72 ss.

Treu T., La contrattazione collettiva nel pubblico impiego: ambiti e struttura, in Dir. Lav. Rel. Ind., 1994, p. 1 ss.

Treu T., Il conflitto e le regole, in Dir. Lav. Rel. Ind., 2000, p. 285 ss.

Treu T., Contrattazione collettiva, in Enc. Dir., Annali, VI, 2011, p. 227 ss.

TREU T., Il salario minimo: limiti della supplenza giurisprudenziale e prospettive, in Giur. It., 2015, p. 743 ss.

TReu T., La questione salariale: legislazione sui minimi e contrattazione collettiva, in WP CSDLE “Massimo D'Antona”.IT, 386/2019.

TREU T., Regole e procedure nelle relazioni industriali: retaggi storici e criticità da affrontare, in WP CSDLE "Massimo D'Antona”.IT, 396/2019.

TReu T., Un segnale unitario per il futuro, in Il Sole 24Ore, 5 giugno 2019.

Tullini P., Finanziamenti pubblici alle imprese e «clausole sociali», in Riv. Trim. Dir. Proc. Civ., 1990, p. 33 ss.

Tuldini P., L'ambito residuo (o surrettizio) di applicazione dell'art. 2070 c.c., in Riv. It. Dir. lav., 1993, II, p. 692 ss. 
TulLiNI P., La revisione del sistema di relazioni sindacali nelle banche, in Dir. Prat. Lav., 1998, 23, p. 1495 ss.

Tullini P., Concorrenza ed equità nel mercato europeo: una scommessa difficile (ma necessaria) per il diritto del lavoro, in Riv. It. Dir. Lav., 2018, I, p. 199 ss.

Tullini P., L'economia digitale alla prova dell'interesse collettivo, in Lab. Law. Iss., 2018, 1, p. 15 ss.

TuRsi A., Sindacati e Cobas: il contratto collettivo "conteso", in Riv. It. Dir. Lav., 1990, I, p. 318 ss.

TURSI A., L'accordo del 31 maggio su rappresentanza e rappresentatività per la stipula dei Ccnl: appunti in tema di rappresentatività, legittimazione negoziale, efficacia soggettiva e contrasto agli "accordi separati”, in Dir. Rel. Ind., 2013, p. 642 ss.

Vallebona A., Il contratto collettivo di lavoro nei Paesi della Comunità europea, Rimini, Maggioli Editore, 1982.

Vallebona A., Veri e falsi limiti alla libertà del datore di scegliere il contratto collettivo, in Riv. It. Dir. Lav., 1990, II, pp. 62 ss.

Vallebona A., Autonomia collettiva e occupazione: l'efficacia soggettiva del contratto collettivo, in Dir. Lav. Rel. Ind., 1997, p. 381 ss.

Vallebona A., Alchimie del legislatore e occhiali del giurista nella riforma della contrattazione collettiva con le pubbliche amministrazioni, in Riv. It. Dir. Lav., 1998, II, p. 51 ss.

Vallebona A., Giochi di parole sull'erga omnes, in Mass. Giur. Lav., 2015, p. 490 ss.

VANDAELE K., Belgium: stability on the surface, mounting tensions beneath, in Müller T., Vandaele K., Waddington J., Collective bargaining in Europe: towards an endgame, I, Brussels, Etui, 2019, p. 53 ss.

VANNES V., Identification de la commission paritaire compétente envers les entreprises concernées, in Orientations, 2010, 4, p. 1 ss.

VARDARo G., Il mutamento della funzione del contratto collettivo, in Dir. Lav. Rel. Ind., 1983, p. 719 ss.

Vardaro G., Contrattazione collettiva e sistema giuridico, Napoli, Jovene, 1984.

VARDARo G., Differenze di funzioni e di livelli fra contratti collettivi, in Lav. Dir., 1987, p. 229 ss.

Varva S., Le clausole sociali, in Carinci M.T., Cester C., Mattarolo M.G., SCARPELli F. (a cura di), Tutela e sicurezza del lavoro negli appalti privati e pubblici. Inquadramento giuridico ed effettività, Torino, Utet, 2011, p. 335 ss. 
Veneziani B., Il sindacato dalla rappresentanza alla rappresentatività, in Dir. Lav. Rel. Ind., 1989, p. 408 ss.

Veneziani B., Stato e autonomia collettiva. Diritto sindacale comparato, Bari, Cacucci, 1992.

VILla E., Crisi della funzione anticoncorrenziale del contratto collettivo nazionale, in Lassandari A., Martelloni F., Tullini P., Zoli C. (a cura di), La contrattazione collettiva nello spazio economico globale, Bologna, Bononia University Press, 2017, p. 73 ss.

VILlalon J.C., Compendio de derecho del trabajo, XI ed., Madrid, Tecnos, 2018.

Villalon J.C. (dir.), La negociación colectiva en Europa. Una perspectiva transversal, Madrid, Ministerio del Trabajo, Migraciones y Seguridad Social, Subdirección General de Información Administrativa y Publicaciones, 2019.

Vincenzi G., Flammia R., Le norme transitorie per garantire minimi di trattamento economico e normativo ai lavoratori, in Mass. Giur. Lav., 1959, p. 115 ss.

Viscomi A., La contrattazione collettiva nazionale, in Zoppoli L. (a cura di), Ideologia e tecnica nella riforma del lavoro pubblico, Napoli, Editoriale Scientifica, 2009, p. 41 ss.

VIscomi A., Prime note sul protocollo 31 maggio 2013, in Riv. It. Dir. Lav., 2013, I, p. 749 ss.

Viscomi A., Soggettività sindacale e categorie contrattuali, in Riv. Giur. Lav., 2014, I, p. 69 ss.

Vitaletti M., La rappresentatività sindacale "utile". Cosa resta del T.U. del 2014, in Dir. Lav. Merc., 2018, p. 37 ss.

WaRdY R., Categories, in Routledge Encyclopaedia.

Weвв S. е B., La democrazia industriale, Torino, Utet, 1912.

Wolfe M., The concept of economic sector, in Quart. J. Eco., 1955, 3, p. 402 ss.

ZiLIo Grandi G., La retribuzione: fonti, struttura, funzioni, Napoli, Jovene, 1996.

Zilio Grandi G., La contrattazione collettiva nelle reti di imprese: spunti per una efficace implementazione, in Giur. It., 2017, p. 1482 ss.

Zoli C., Gli obblighi a trattare nel sistema dei rapporti collettivi, Padova, Cedam, 1992.

Zoli C., Il corrispettivo della prestazione lavorativa, in Nogler L., TRemoLADA M., Zoli C. (a cura di), La riforma della posizione giuridica del socio lavoratore di cooperativa, in Nuove Leggi Civ., 2002, p. 408 ss. 
Zoli C., Contratto collettivo come fonte e contrattazione collettiva come sistema di produzione di regole, in PERSIANI M. (a cura di), Le fonti del diritto del lavoro, in CARINCI F., Persiani M. (dir. da), Trattato di diritto del lavoro, Padova, Cedam, 2010, p. 487 ss.

Zoli C., La struttura della contrattazione collettiva nel lavoro pubblico, in Fiorillo L., Perulli A. (dir. da), Il nuovo diritto del lavoro, 1, Il lavoro alle dipendenze delle amministrazioni pubbliche, Torino, Giappichelli, 2013, p. 161 ss.

Zoli C., La fruizione dei beni culturali quale servizio pubblico essenziale: il decreto legge 20 settembre 2015, n. 146 in tema di sciopero, in Aedon, 2015, 3, p. 6 ss.

Zoli C., Cusa E., Art. 3 (Premessa), in Nogler L., Tremolada M., Zoli C. (a cura di), La riforma della posizione giuridica del socio lavoratore di cooperativa, Nuove Leggi Civ., 2002, p. 407 s.

Zoppoli A., La titolarità sindacale del diritto di sciopero, Napoli, Jovene, 2006.

Zoppoli A., Sulle ragioni, oggi, di una regolazione a tutto tondo (del potere sindacale e) della contrattazione collettiva (in particolare, sulla proposta di legge di «Diritti lavori mercati»), in AA.VV., L'attuazione degli articoli 39 e 46 della Costituzione. Tre proposte a confronto, Quaderni Riv. Giur. Lav., Roma, Ediesse, 2016, p. 151 ss.

Zoppoli A., Rappresentanze sindacali e rappresentatività, le insidie del tempo, in Dir. Lav. Merc., 2018, p. 351 ss.

Zoppoli L., I comparti pubblici dopo la riforma del rapporto di lavoro, in Lav. Inf., 1993, 10, p. 5 ss.

Zoppoli L., Delfino M., La rappresentanza delle pubbliche amministrazio$n i$, in Proia G. (a cura di), Organizzazione sindacale e contrattazione collettiva, in CARINCI F., Persiani M. (dir. da), Trattato di diritto del lavoro, Padova, Cedam, 2014, p. 1033 ss.

Zoppoli L., Zoppoli A., Delfino M. (a cura di), Una nuova Costituzione per il sistema di relazioni sindacali?, Napoli, Editoriale Scientifica, 2014. 



\section{SOMMARIO}

\section{CAPITOLO I \\ LIBERTÀ SINDACALE E CATEGORIA CONTRATTUALE}

1. Il problema della categoria 5

2. Pluralità di significati del lemma categoria nel diritto sindacale 8

3. Categoria professionale e categoria sindacale 11

4. Categoria contrattuale 18

5. Le tipizzazioni dell'autonomia collettiva 24

6. Principio di libertà sindacale e definizione delle unità negoziali 29

6.1. La giurisprudenza costituzionale sulla legge Vigorelli 30

6.2. L'affermazione di una nozione volontaristica di categoria 34

7. La sovrapposizione tra gli ambiti di applicazione dei contratti collettivi di categoria: un fenomeno relativamente nuovo 38

8. Inquadramento delle questioni da affrontare e struttura del lavoro 43

\section{CAPITOLO II \\ LA PROSPETTIVA INTERNA ALLE RELAZIONI INDUSTRIALI}

1. Le unità negoziali nella contrattazione collettiva del settore privato: considerazioni introduttive

2. Il problema del criterio di collegamento tra le unità negoziali e i rapporti di lavoro 53

3. La contrattazione collettiva di categoria al bivio 58

3.1. L'archivio Cnel dei contratti collettivi: cenni essenziali 59

3.2. Dal pluralismo al caos? $\quad 61$

3.2.1. Il grattacapo degli accordi "pirata" 65

3.2.2. Il dumping contrattuale interno al sistema di rappresentanza "confederale" 71

4. Il percorso a ostacoli per una riorganizzazione del sistema contrattuale 75 


\section{CAPITOLO III \\ AUTONOMIA DELLE PARTI ED INTERVENTO DELLA LEGGE NELLA DEFINIZIONE DELLE UNITÀ NEGOZIALI}

1. Libertà di definizione delle unità negoziali e certezza giuridica: considerazioni sull'esigenza di pervenire ad un contemperamento

2. Il criterio oggettivo di collegamento tra le unità negoziali e i rapporti di lavoro

3. I meccanismi di definizione delle unità negoziali: una possibile bipartizione

3.1. Meccanismi antecedenti (che operano in una fase che precede lo svolgimento della contrattazione collettiva)

3.2. Meccanismi contestuali (in cui la categoria è fissata con la stipulazione dei contratti collettivi)

3.3. Considerazioni conclusive

4. Rassegna delle soluzioni tecniche avanzate per la definizione delle unità negoziali nelle proposte di attuazione dell'art. 39 Cost.

4.1. Dall'entrata in vigore della Costituzione ai primi anni Sessanta

4.2. Riprende il dibattito sulla legge sindacale: gli anni Ottanta e Novanta

4.3. La Carta dei diritti della Cgil e le proposte della rivista $D L M$ e del gruppo Freccia Rossa

5. La definizione dei comparti di contrattazione nel settore pubblico

5.1. Evoluzione delle discipline legislative e della corrispondente attività negoziale

5.2. La limitata "sovranità" delle organizzazioni sindacali nella definizione dei comparti

6. Sciopero nei servizi pubblici essenziali e prestazioni indispensabili

6.1. Il quadro normativo: cenni essenziali

\section{CAPITOLO IV \\ PARAMETRI DEL TRATTAMENTO \\ E RINVII ALL’AUTONOMIA COLLETTIVA}

1. Parametro del trattamento economico e normativo e rinvii all'autonomia collettiva per determinarlo

2. Un modello di intervento "leggero" sul sistema sindacale

3. Le clausole sul campo di applicazione dei contratti collettivi: problemi interpretativi

4. La selezione dei contratti-parametro: il problema di un ambito residuo di vigenza dell'art. 2070 c.c.

5. Pluralità di contratti collettivi con ambito di applicazione sovrapposto. L'esigenza di dotarsi di più adeguati criteri selettivi

6. Selezioni e misurazioni nella maggior rappresentatività comparata

6.1. La comparazione tra contratti collettivi con ambito di applicazione esattamente coincidente 
6.1.1. Critica

6.1.2. Spunti per una ricostruzione alternativa

6.2. La misurazione effettiva della rappresentatività: un cantiere aperto nelle relazioni industriali

6.3. Le rilevazioni Cnel-Inps sull'applicazione dei contratti collettivi alle imprese e ai lavoratori ad esse addetti

Conclusioni

Bibliografia 

1. Coli U., Collegia et sodalitates, 1913.

2. Donatelli I., La "consortia" di Avesa, 1914.

3. VAlenza P., Il diritto di usufrutto nelle leggi sulle tasse del registro, 1915.

4. Zingali G., La statistica della criminalità, 1916.

5. Tumedei C., La separazione dei beni ereditari, 1917.

6. Albertoni A., L"Apokeryxis", 1923.

7. Salvi F., La cessione dei beni ai creditori, 1947.

8. Milani F., Distinzioni delle servitù prediali, 1948.

9. FAssò G., I "quattro autori" del Vico, 1949.

10. Ferri L., La trascrizione degli acquisti "mortis causa" e problemi connessi, 1951.

11. Rossi G., La "Summa arboris actionum" di Ponzio da Ylerda, 1951.

12. Poggeschi R., Le associazioni e gli altri gruppi con autonomia patrimoniale nel processo, 1951.

13. Matteucci N., Antonio Gramsci e la filosofia della prassi, 1951.

14. Forchielli P., I contratti reali, 1952.

15. SALVI F., Il possesso di stato familiare, 1952.

16. FAssò G., La storia come esperienza giuridica, 1953.

17. Palazzini Finetti L., Storia della ricerca delle interpolazioni nel Corpus iuris giustinianeo, 1953.

18. Rossi G., Consilium sapientis iudiciale, 1958.

19. Mancini G.F., La responsabilità contrattuale del prestatore di lavoro, 1957.

20. Ferri L., L'autonomia privata, 1959.

21. Torelli P., Scritti di storia del diritto italiano, 1959.

22. Santini G., I Comuni di Valle del medioevo. La Costituzione federale del "Frignano", 1960.

23. Gianniti F., I reati della stessa indole, 1959.

24. GHEzz G., La prestazione di lavoro nella comunità familiare, 1960.

25. NARDi E., Case "infestate da spiriti" e diritto romano e moderno, 1960.

26. FERR L., Rinunzia e rifiuto nel diritto privato, 1960.

27. GHEzz G., La responsabilità contrattuale delle associazioni sindacali, 1963.

28. Bonsignori A., Espropriazione della quota di società a responsabilità limitata, 1961.

29. Redenti E., Scritti e discorsi giuridici di un mezzo secolo, vol. I, Intorno al diritto processuale, 1962.

30. Redenti E., Scritti e discorsi giuridici di un mezzo secolo, vol. II, Intorno al diritto sostanziale, 1962.

31. Gualandi A., Spese e danni nel processo civile, 1962.

32. Bonsignori A., Assegnazione forzata e distribuzione del ricavato, 1960.

33. Mancini G.F., Il recesso unilaterale e $i$ rapporti di lavoro, vol. I, Individuazione della fattispecie. Il recesso ordinario, 1962.

34. NARDI E., Rabelais e il diritto romano, 1962.

35. Romagnoli U., Il contratto collettivo di impresa, 1963.

36. SANTINI G., I "comuni di pieve" nel medioevo italiano, 1964.

37. RUDAN M., Il contratto di tirocinio, 1966.

38. Bonini R., I "libride cognitionibus" di Callistrato. Ricerche sull'elaborazione giurisprudenziale della "cognitio extra ordinem", 1964.

39. Colliva P., Ricerche sul principio di legalità nell'amministrazione del Regno di Sicilia al tempo di Federico II, 1964.

40. MengozZi P., L'agenzia di approvvigionamento dell'Euratom, 1964.

41. Scritti minori di Antonio Cicu, tomi I e II, Scritti di teoria generale del diritto - Diritto di famiglia, 1965.

42. Scritti minori di Antonio Cicu, Successioni e donazioni. Studi vari, 1965.

43. Sacchi Morsiani G., Il potere amministrativo delle Comunità europee e le posizioni giuridiche dei privati, I, 1965.

44. GHEzzi G., La mora del creditore nel rapporto di lavoro, 1965.

45. Roversi Monaco F.A., Enti di gestione. Struttura, funzioni, limiti, 1967.

46. Gianniti F., L'oggetto materiale del reato, 1966. 
47. Mengozzi P., L'efficacia in Italia di atti stranieri di potestà pubblica su beni privati, 1967.

48. Romagnoli U., La prestazione di lavoro nel contratto di società, 1967.

49. Montuschi L., I limiti legali nella conclusione del contratto di lavoro, 1967.

50. Ranieri S., Scritti e discorsi vari, vol. I, Scritti di diritto penale, 1968.

51. Ranieri S., Scritti e discorsi vari, vol. II, Scritti di procedura penale, 1968.

52. Bonini R., Ricerche di diritto giustinianeo, 1968.

53. SANTINI G., Ricerche sulle "Exceptiones legum romanorum", 1969.

54. Lo Castro G., La qualificazione giuridica delle deliberazioni conciliari delle fonti del diritto canonico, 1970.

55. SaCChi Morsiani G., Il potere amministrativo delle Comunità europee e le posizioni giuridiche dei privati, II, 1970.

56. Roversi Monaco F.A., La delegazione amministrativa nel quadro dell'ordinamento regionale, 1970.

57. Gianniti F., Studi sulla corruzione del pubblico ufficiale, 1970.

58. De Vergottini G., Indirizzo politico della difesa e sistema costituzionale, 1971.

59. Mengozzi P., Il regime giuridico internazionale del fondo marino, 1971.

60. CARINCI F., Il conflitto collettivo nella giurisprudenza costituzionale, 1971.

61. Osti G., Scritti giuridici, voll. I e II, 1973.

62. Zueldi F., Servizi pubblici e attività imprenditoriale, 1973.

63. Pergolesi F., Sistema delle fonti normative, 1973.

64. Montuschi L., Potere disciplinare e rapporto di lavoro, 1973.

65. Pattaro E., Il pensiero giuridico di L.A. Muratori tra metodologia e politica, 1974.

66. PINI G., Arbitrato e lavori pubblici, 1974.

67. CARPI F., L'efficacia "ultra partes" della sentenza civile, 1974.

68. De Vergottini G., Lo "Shadow cabinet", 1973.

69. Paolucci L.F., La mutualità nelle cooperative, 1974.

70. De Gennaro A., Crocianesimo e cultura giuridica italiana, 1974.

71. Stortoni L., L'abuso di potere nel diritto penale, 1978.

72. Gianniti F., Prospettive criminologiche e processo penale, 1977.

73. Bonvicini D., Le "joint ventures": tecnica giuridica e prassi societaria, 1977.

74. De Vergottini G., Scritti di storia del diritto italiano, voll. I, II, III, 1977.

75. Lambertini R., I caratteri della Novella 118 di Giustiniano, 1977.

76. Dalla D., L'incapacità sessuale in diritto romano, 1978.

77. Di Pietro A., Lineamenti di una teoria giuridica dell'imposta sull'incremento di valore degli immobili, 1978.

78. MazZaCuva N., La tutela penale del segreto industriale, 1979.

79. Romanelli G., Profilo del noleggio, 1979.

80. Borghesi D., Il contenzioso in materia di eleggibilità, 1979.

81. Dalla Torre G., L'attività assistenziale della Chiesa nell'ordinamento italiano, 1979.

82. CARPI F., La provvisoria esecutorietà della sentenza, 1979.

83. Alleva P., Il campo di applicazione dello statuto dei lavoratori, 1980.

84. Puliatti S., Ricerche sulla legislazione "regionale" di Giustiniano, 1980.

85. FAssò G., Scritti di filosofia del diritto, voll. I, II, III, 1982.

86. SGUbBi F., Uno studio sulla tutela penale del patrimonio, 1980.

87. Lambertini R., Plagium, 1980.

88. Dalla D., Senatus consultum Silanianum, 1980.

89. VANDELLI L., L'ordinamento regionale spagnolo, 1980.

90. NARDI E., L'otre dei parricidi e le bestie incluse, 1980.

91. Pellicanò A., Causa del contratto e circolazione dei beni, 1981.

92. Giardini D., Politica e amministrazione nello Stato fondato sul decentramento, 1981.

93. Bortolotti D., Potere pubblico e ambiente, 1981.

94. Roffi R., Contributo per una teoria delle presunzioni nel diritto amministrativo, 1982.

95. Alessi R., Scritti minori, 1981.

96. Bassanelli Sommariva G., L'imperatore unico creatore ed interprete delle leggi e l'autonomia del giudice nel diritto giustinianeo, 1983.

97. Zanotтi A., Cultura giuridica del Seicento e jus publicum ecclesiasticum nell'opera del cardinal Giovanni Battista De Luca, 1983.

98. Illuminati G., La disciplina processuale delle intercettazioni, 1983.

99. Toniatti R., Costituzione e direzione della politica estera negli Stati Uniti d'America, 1983.

100. NARDI E., Squilibrio e deficienza mentale in diritto romano, 1983. 
101. Dalla D., Praemium emancipationis, 1983.

102. Mazzacuva N., Il disvalore di evento nell'illecito penale - L'illecito commissivo doloso e colposo, 1983.

103. Studi in onore di Tito Carnacini. I. Studi di diritto costituzionale, civile, del lavoro, commerciale, 1983.

104. CAIA G., Stato e autonomie locali nella gestione dell'energia, 1984.

105. Baratti G., Contributo allo studio della sanzione amministrativa, 1984.

106. Bовтоцотті D., Attività preparatoria e funzione amministrativa, 1984.

107. Puliatti S., Ricerche sulle novelle di Giustino II. La legislazione imperiale da Giustiniano I a Giustino II, 1984.

108. Lambertini R., La problematica della commorienza nell'elaborazione giuridica romana, 1984.

109. ZueLLI F., Le collegialità amministrative, 1985.

110. Pedrazzoli M., Democrazia industriale e subordinazione, 1985.

111. ZanotTi M., Profili dogmatici dell'illecito plurisoggettivo, 1985.

112. Ruffolo U., Interessi collettivi o diffusi e tutela del consumatore, I, 1985.

113. BIAGI M., Sindacato democrazia e diritto, 1986.

114. Insolera G., Problemi di struttura del concorso di persone nel reato, 1986.

115. Malagù L., Esecuzione forzata e diritto di famiglia, 1986.

116. Ricci G.F., La connessione nel processo esecutivo, 1986.

117. ZANOTTI A., Il concordato austriaco del 1855, 1986.

118. Selmini R., Profili di uno studio storico sull'infanticidio, 1987.

119. DaLla D., "Ubi venus mutatur", 1987.

120. Zunarelli S., La nozione di vettore, 1987.

121. ZoLI C., La tutela delle posizioni "strumentali" del lavoratore, 1988.

122. Cavina M., Dottrine giuridiche e strutture sociali padane nella prima età moderna, 1988.

123. Califano L., Innovazione e conformità nel sistema regionale spagnolo, 1988.

124. SARTi N., Gli statuti della società dei notai di Bologna dell'anno 1336 (contributo allo studio di una corporazione cittadina), 1988.

125. SCARPONi S., Riduzione e gestione flessibile del tempo di lavoro, 1988.

126. Bernardini M., Contenuto della proprietà edilizia, 1988.

127. La Torre M., La "lotta contro il diritto soggettivo". Karl Larenz - la dottrina giuridica nazionalsocialista, 1988.

128. Garcia De Enterria J., Le obbligazioni convertibili in azioni, 1989.

129. Biagi Guerini R., Famiglia e Costituzione, 1989.

130. CAIA G., Arbitrati e modelli arbitrali nel diritto amministrativo, 1989.

131. Magagni M., La prestazione caratteristica nella Convenzione di Roma del 19 giugno 1980, 1989.

132. Petroni L., La disciplina pubblicistica dell'innovazione tecnologica in Francia, 1990.

133. ZanotTi A., Le manipolazioni genetiche e il diritto della Chiesa, 1990.

134. SARTOR G., Le applicazioni giuridiche dell'intelligenza artificiale, 1990.

135. Rossi L.S., Il "buon funzionamento del mercato comune". Delimitazione dei poteri fra CEE e Stati membri, 1990.

136. LuCHEтti G., La legittimazione dei figli naturali nelle fonti tardo imperiali e giustinianee, 1990.

137. SARTI N., Un giurista tra Azzone e Accursio, 1990.

138. Gustapane A., La tutela globale dell'ambiente, 1991.

139. Bottari C., Principi costituzionali e assistenza sanitaria, 1991.

140. Donini M., Illecito e colpevolezza nell'imputazione del reato, 1991.

141. Perulli A., Il potere direttivo dell'imprenditore, 1992.

142. VANDELLI L. (a cura di), Le forme associative tra enti territoriali, 1992.

143. GASPARRI P., Institutiones iuris publici, 1992.

144. CAPUZzo E., Dal nesso asburgico alla sovranità italiana, 1992.

145. Biavati P., Accertamento dei fatti e tecniche probatorie nel processo comunitario, 1992.

146. Ferrari F., Atipicità dell'illecito civile. Una comparazione, 1992.

147. Gustapane A., Sartor G., Verardi C.M., Valutazione di impatto ambientale. Profili normativi e metodologie informatiche, 1992.

148. Orlandi R., Atti e informazioni della autorità amministrativa nel processo penale. Contributo allo studio delle prove extracostituite, 1992.

149. Carpani G., Le aziende degli enti locali. Vigilanza e controlli, 1992. 
150. Musso A., Concorrenza ed integrazione nei contratti di subfornitura industriale, 1993.

151. Donini M., Il delitto contravvenzionale. "Culpa iuris" e oggetto del dolo nei reati a condotta neutra, 1993.

152. Califano Placci L., Le commissioni parlamentari bicamerali nella crisi del bicameralismo italiano, 1993.

153. FoRNASARI G., Il concetto di economia pubblica nel diritto penale. Spunti esegetici e prospettive di riforma, 1994

154. ManZINI P., L'esclusione della concorrenza nel diritto antitrust italiano, 1994.

155. Ттмотео M., Le successioni nel diritto cinese. Evoluzione storica ed assetto attuale, 1994

156. Sesta M. (a cura di), Per i cinquant'anni del codice civile, 1994.

157. Tullini P., Contributo alla teoria del licenziamento per giusta causa, 1994.

158. Rescigno F., Disfunzioni e prospettive di riforma del bicameralismo italiano: la camera delle regioni, 1995.

159. Lugaresi N., Le acque pubbliche. Profili dominicali, di tutela, di gestione, 1995.

160. SARTI N., Maximum dirimendarum causarum remedium. Il giuramento di calunnia nella dottrina civilistica dei secoli XI-XIII, 1995.

161. Colliva P., Scritti minori, 1996.

162. Dugato M., Atipicità e funzionalizzazione nell'attività amministrativa per contratti, 1996.

163. GaRdini G., La comunicazione degli atti amministrativi. Uno studio alla luce della legge 7 agosto 1990, n. 241, 1996.

164. ManZINI P., I costi ambientali nel diritto internazionale, 1996.

165. MiтTica M.P., Il divenire dell'ordine. L'interazione normativa nella società omerica, 1996.

166. LuchetTi G., La legislazione imperiale nelle Istituzioni di Giustiniano, 1996.

167. La Torre M., Disavventure del diritto soggettivo. Una vicenda teorica, 1996.

168. Camon A., Le intercettazioni nel processo penale, 1996.

169. Mancini S., Minoranze autoctone e Stato. Tra composizione dei conflitti e secessione, 1996.

170. Zanobetti Pagnetti A., La non comparizione davanti alla Corte internazionale di giustizia, 1996.

171. Bricola F., Scritti di diritto penale. Vol. I, Dottrine generali, Teoria del reato e sistema sanzionatorio. Vol. II, Parte speciale e legislazione complementare, Diritto penale dell'economia, 1997.

172. Graziosi A., La sentenza di divorzio, 1997.

173. Mantovani M., Il principio di affidamento nella teoria del reato colposo, 1997.

174. Biavati P., Giurisdizione civile, territorio e ordinamento aperto, 1997.

175. Rossi G. (1916-1986), Studi e testi di storia giuridica medievale, a cura di Giovanni Gualandi e Nicoletta Sarti, 1997.

176. Pellegrini S., La litigiosità in Italia. Un'analisi sociologico-giuridica, 1997.

177. Boni G., La rilevanza del diritto dello Stato nell'ordinamento canonico. In particolare la canonizatio legum civilium, 1998.

178. Scritti in onore di Giuseppe Federico Mancini. Vol. I, Diritto del lavoro, 1998

179. Scritti in onore di Giuseppe Federico Mancini. Vol. II, Diritto dell'Unione europea, 1998

180. Rossi A., Il GEIE nell'ordinamento italiano. Criteri di integrazione della disciplina, 1998.

181. Bongiovanni G., Reine Rechtslehre e dottrina giuridica dello Stato. H. Kelsen e la Costituzione austriaca del 1920, 1998.

182. Caputo G., Scritti minori, 1998.

183. GARRIDO J.M., Preferenza e proporzionalità nella tutela del credito, 1998.

184. Bellodi Ansaloni A., Ricerche sulla contumacia nelle cognitiones extra ordinem, I, 1998.

185. Franciosi E., Riforme istituzionali e funzioni giurisdizionali nelle Novelle di Giustiniano. Studi su nov. 13 e nov. 80, 1998.

186. Cattabriga C., La Corte di giustizia e il processo decisionale politico comunitario, 1998.

187. Mancini L., Immigrazione musulmana e cultura giuridica. Osservazioni empiriche su due comunità di egiziani, 1998.

188. Gustapane A., L'autonomia e l'indipendenza della magistratura ordinaria nel sistema costituzionale italiano. dagli albori dello Statuto Albertino al crepuscolo della bicamerale, premessa di Giuseppe De Vergottini, 1999.

189. RICCI G.F., Le prove atipiche, 1999.

190. Canestrari S., Dolo eventuale e colpa cosciente. Ai confini tra dolo e colpa nella struttura delle tipologie delittuose, 1999.

191. Fassò G., La legge della ragione. Ristampa, a cura di Carla Faralli, Enrico Pattaro, Giampaolo Zucchini, 1999. 
192. FAssò G., La democrazia in Grecia. Ristampa, a cura di Carla Faralli, Enrico Pattaro, Giampaolo Zucchini, 1999.

193. Scarciglia R., La motivazione dell'atto amministrativo. Profili ricostruttivi e analisi comparatistica, 1999.

194. BRiguglio F., "Fideiussoribus succurri solet", 1999.

195. Maltoni A., Tutela dei consumatori e libera circolazione delle merci nella giurisprudenza della Corte di giustizia, profili costituzionali, prefazione di Augusto Barbera, 1999.

196. FondaRoLi D., Illecito penale e riparazione del danno, 1999.

197. Rossi L.S., Le convenzioni fra gli Stati membri dell'Unione europea, 2000.

198. Gragnoli E., Profili dell'interpretazione dei contratti collettivi, 2000.

199. Boni G., La rilevanza del diritto secolare nella disciplina del matrimonio canonico, 2000.

200. Lugaresi N., Internet, privacy e pubblici poteri negli Stati Uniti, 2000.

201. Lalatta Costerbosa M., Ragione e tradizione. Il pensiero giuridico ed etico-politico di Wilehlmvon Humboldt, 2000.

202. Semeraro P., I delitti di millantato credito e traffico di influenza, 2000.

203. Verza A., La neutralità impossibile. Uno studio sulle teorie liberali contemporanee, 2000.

204. Lolli A., L'atto amministrativo nell'ordinamento democratico. Studio sulla qualificazione giuridica, 2000.

205. BusetTo M.L., Giudice penale e sentenza dichiarativa di fallimento, 2000.

206. Campanella P., Rappresentatività sindacale: fattispecie ed effetti, 2000.

207. Bricola F., Scritti di diritto penale. Opere monografiche, 2000.

208. LASSANDARI A., Il contratto collettivo aziendale e decentrato, 2001.

209. Bianco A., Il finanziamento della politica in Italia, 2001.

210. Raffi A., Sciopero nei servizi pubblici essenziali. Orientamenti della Commissione di garanzia, 2001.

211. Piergigli V., Lingue minoritarie e identità culturali, 2001.

212. CAFARO S., Unione monetaria e coordinamento delle politiche economiche. Il difficile equilibrio tra modelli antagonisti di integrazione europea, 2001.

213. Morrone A., Il custode della ragionevolezza, 2001.

214. Masutti A., La liberalizzazione dei trasporti in Europa. Il caso del trasporto postale, 2002.

215. Zanotti A., Orlando F., L'itinerario canonistico di Giuseppe Caputo, 2002.

216. Lupor M.A., Conflitti transnazionali di giurisdizioni. Vol. I, Policies, metodi, criteri di collegamento. Vol. II, Parallel proceedings, 2002.

217. LolLi A., I limiti soggettivi del giudicato amministrativo. Stabilità del giudicato e difesa del terzo nel processo amministrativo, 2002.

218. Curi F., Tertium datur. Dal Common Law al Civil Law per una scomposizione tripartita dell'elemento soggettivo del reato, 2003.

219. Cottignola G., Studi sul pilotaggio marittimo, 2003.

220. GARDINI G., L'imparzialità amministrativa tra indirizzo e gestione. Organizzazione e ruolo della dirigenza pubblica nell'amministrazione contemporanea, 2003.

221. Cevenini C., Virtual enterprises. Legal issues of the on-line collaboration between undertakings, 2003.

222. Monducci J., Diritto della persona e trattamento dei dati particolari, 2003.

223. Villecco Bettelli A., L'efficacia delle prove informatiche, 2004.

224. Zucconi Galdi Fonseca E., La convenzione arbitrale rituale rispetto ai terzi, 2004.

225. Brighi R., Norme e conoscenza: dal testo giuridico al metadato, 2004.

226. Luchetti G., Nuove ricerche sulle istituzioni di Giustiniano, 2004.

227. Studi in memoria di Angelo Bonsignori, voll. I, II, 2004.

228. Piperata G., Tipicità e autonomia nei servizi pubblici locali, 2005.

229. Canestrari S., Foffani L. (a cura di), Il diritto penale nella prospettiva europea. Quali politiche criminali per l'Europa? Atti del Convegno organizzato dall'Associazione Franco Bricola (Bologna, 28 febbraio-2 marzo 2002), 2005.

230. Мемmo D., Miconi S. (a cura di), Broadcasting regulation: market entry and licensing. Regolamentazione dell'attività radiotelevisiva: accesso al mercato e sistema di licenze. Global Classroom Seminar, 2006.

230. bis Briguglio F., Studi sul procurator, 2007.

231. Querzola L., La tutela anticipatoria fra procedimento cautelare e giudizio di merito, 2006.

232. TAROZZI S., Ricerche in tema di registrazione e certificazione del documento nel periodo postclassico, 2006.

233. Bотті F., L'eutanasia in Svizzera, 2007. 
234. Fondaroli D., Le ipotesi speciali di confisca nel sistema penale, 2007.

235. Alagna R., Tipicità e riformulazione del reato, 2007.

236. Giovannini M., Amministrazioni pubbliche e risoluzione alternativa delle controversie, 2007.

237. Montalti M., Orientamento sessuale e costituzione decostruita. Storia comparata di un diritto fondamentale, 2007.

238. Tordini Cagli S., Principio di autodeterminazione e consenso dell'avente diritto, 2008.

239. Legnani Annichini A., La mercanzia di Bologna. Gli statuti del 1436 e le riformagioni quattrocentesche, 2008.

240. LolLI A., L'amministrazione attraverso strumenti economici, 2008.

241. VACCARELla M., Titolarità e funzione nel regime dei beni civici, 2008.

242. Tubertini C., Pubblica amministrazione e garanzia dei livelli essenziali delle prestazioni, 2008.

243. Fioniglio G., Il diritto alla privacy. Nuove frontiere nell'era di Internet, 2008.

244. Вотті F., Manipolazioni del corpo e mutilazioni genitali femminili, 2009.

245. Nisco A., Controlli sul mercato finanziario e responsabilità penale. Posizioni di garanzia e tutela del risparmio, 2009.

246. Zanobetti Pagnetti A., Il rapporto internazionale di lavoro marittimo, 2008.

247. Mаттіо $\mathrm{F}$., Ricerche sulla formazione della categoria dei cosiddetti quasi delitti, 2010.

248. Bertaccini D., La politica di polizia, 2009.

249. Astrologo A., Le cause di non punibilità. Un percorso tra nuovi orientamenti interpretativi e perenni incertezze dogmatiche, 2009.

250. DI MARIA S., La cancelleria imperiale e $i$ giuristi classici: "Reverentia antiquitatis" e nuove prospettive nella legislazione giustinianea del codice, 2010.

251. V ALENTINI E., La domanda cautelare nel sistema delle cautele personali, 2010.

252. Querzola L., Il processo minorile in dimensione europea, 2010.

253. Bologna C., Stato federale e "national interest". Le istanze unitarie nell'esperienza statunitense, 2010.

254. Rasia C., Tutela giudiziale europea e arbitrato, 2010.

255. Zucconi Galli Fonseca E., Pregiudizialità e rinvio (Contributo allo studio dei limiti soggettivi dell'accertamento), 2011.

256. Bellodi Ansaloni A., Ad eruendam veritatem. Profili metodologici e processuali della quaestio per tormenta, 2011.

257. Pontoriero I., Il prestito marittimo in diritto romano, 2011.

258. Giustizia senza confini. Studi offerti a Federico Carpi, 2012.

259. Gustapane A., Il ruolo del pubblico ministero nella Costituzione italiana, 2012.

260. Cainniello M., Premesse per una teoria del pregiudizio effettivo nelle invalidità processuali penali, 2012.

261. Briguglio F., Il Codice Veronese in trasparenza. Genesi e formazione del testo delle Istituzioni di Gaio, 2012.

262. Valentini E., La domanda cautelare nel sistema delle cautele personali, Nuova edizione, 2012.

263. TASsinari D., Nemo tenetur se detegere. La libertà dalle autoincriminazioni nella struttura del reato, 2012.

264. Martelloni F., Lavoro coordinato e subordinazione. L'interferenza delle collaborazioni a progetto, 2012.

265. Roversi-Monaco F. (a cura di), Università e riforme. L'organizzazione delle Università degli Studi ed il personale accademico nella legge 30 dicembre 2010, n. 240, 2013.

266. TorRe V., La privatizzazione delle fonti di diritto penale, 2013.

267. Raffiotta E.C., Il governo multilivello dell'economia. Studio sulle trasformazioni dello Stato costituzionale in Europa, 2013.

268. Caruso C., La libertà di espressione in azione. Contributo a una teoria costituzionale del discorso pubblico, 2013.

269. Pedrini F., Le "clausole generali". Profili teorici e aspetti costituzionali, 2013.

270. Curi F., Profili penali dello stress lavoro-correlato. L'homo faber nelle organizzazioni complesse, 2013.

271. Casale D., L'idoneità psicofisica del lavoratore pubblico, 2013.

272. Nicodemo S., Le istituzioni della conoscenza nel sistema scolastico, 2013.

273. Legnani Annichini A., "Proxeneta est in tractando». La professione ingrata del mediatore di commercio (secc. XII-XVI), 2013.

274. Monducci J., Il dato genetico tra autodeterminazione informativa e discriminazione genotipica, 2013.

275. Mantovani M., Contributo ad uno studio sul disvalore di azione nel sistema penale vigente, 2014.

276. DE Donno M., Consensualità e interesse pubblico nel governo del territorio, 2015. 
277. Pacilli M., L'abuso dell'appello, 2015.

278. PIšTan Č., Tra democrazia e autoritarismo. Esperienze di giustizia costituzionale nell'Europa centro-orientale e nell'area post-sovietica, 2015.

279. Bellodi Ansaloni A., L'arte dell'avvocato, actor veritatis. Studi di retorica e deontologia forense, 2016.

280. Нохна D., La giustizia criminale napoleonica. A Bologna fra prassi e insegnamento universitario, 2016.

281. Querzola L., L'efficacia dell'attività processuale in un diverso giudizio, 2016.

282. Pieri B., Usurai, ebrei e poteri della Chiesa nei consilia di Paolo da Castro, 2016.

283. Rasia C., La crisi della motivazione nel processo civile, 2016.

284. Drigo C., Le Corti costituzionali tra politica e giurisdizione, 2016.

285. Polacchini F., Doveri costituzionali e principio di solidarietà, 2016.

286. Calcagnile M., Inconferibilità amministrativa e conflitti di interesse nella disciplina dell'accesso alle cariche pubbliche, 2017.

287. VILLA E., La responsabilità solidale come tecnica di tutela del lavoratore, 2017.

288. VINCIERI M., L'integrazione dell'obbligo di sicurezza, 2017.

289. CaSALE D., L'automaticità delle prestazioni previdenziali. Tutele, responsabilità e limiti, 2017.

290. GANARIN M., L'interpretazione autentica nelle attuali dinamiche evolutive del diritto canonico, 2018.

291. LaUs F., Il rapporto collaborativo tra pubblico e privato nella contrattazione pubblica. Unione Europea e ordinamenti nazionali: analisi comparata di modelli e riforme, 2018.

292. Bonacini P., Multa scripsit, nihil tamen reperitur. Niccolò Mattarelli giurista a Modena e Padova (1204 ca.-1314 ca.), 2018.

293. Gabellini E., L'azione arbitrale. Contributo allo studio dell'arbitrabilità dei diritti, 2018.

294. LupoI M.A., Tra flessibilità e semplificazione. Un embrione di case management all'italiana?, 2018.

295. Dallari F., Vincoli espropriativi e perequazione urbanistica. La questione della discrezionalità, 2018.

296. Donini A., Il lavoro attraverso le piattaforme digitali, 2019.

297. Novaro P., Profili giuridici dei residui delle attività antropiche urbane. Gli incerti confini della gestione dei rifiuti urbani, 2019.

298. Matтіоl F., Giustiniano, gli argentarii e le loro attività negoziali. La specialità di un diritto e le vicende della sua formazione, 2019.

299. RafFiotta E.C., Norme d'ordinanza. Contributo a una teoria delle ordinanze emergenziali come fonti normative, 2019.

300. Medina M.H., Servio Sulpicio Rufo: un retrato final desde la perspectiva de Cicerón, 2020.

301. Centamore G., Contrattazione collettiva e pluralità di categorie, 2020. 
Finito di stampare nel mese di maggio 2020 per i tipi di Bononia University Press 